\title{
Retrieving the 3D distribution of leaves in forests using LiDAR
}

\author{
Jing Liu
}





\title{
RETRIEVING THE 3D DISTRIBUTION OF LEAVES IN FORESTS USING LIDAR
}

\author{
DIS SERTATION
}

\begin{abstract}
to obtain
the double-badged degree of doctor

at the University of Twente, Enschede, The Netherlands; and RMIT University, Melbourne, Australia,

on the authority of the rector magnificus, prof.dr. T.T.M. Palstra, for University of Twente on the authority of the Chancellor, Dr Ziggy Switkowski AO; and the Vice-Chancellor and President of RMIT University, Mr Martin Bean CBE, for RMIT University
\end{abstract}

on account of the decision of the joint Doctorate Board, to be publicly defended on Wednesday, May 22, 2019 at 12.45

by

Jing Liu

born on September 2, 1989

in ShaanXi, China 
This dissertation is approved by:

Prof.dr. A.K. Skidmore (supervisor)

Prof.dr. S. Jones (supervisor)

Dr. T. Wang (co-supervisor)

ITC dissertation number 354

ITC, P.O. Box 217, 7500 AE Enschede, The Netherlands

ISBN: 978-90-365-4772-7

DOI: $10.3990 / 1.9789036547727$

Printed by: ITC printing Department

(c) Jing Liu, Enschede, The Netherlands

(c) Cover design by Benno Masselink

All rights reserved. No part of this publication may be reproduced without the prior written permission of the author.

\section{DMIT}

UNIVERSITY

\section{(T) uwvessrrof rwewte}

U1C FACULTY OF GEO-INFORMATION SCIENCE AND EARTH OBSERVATION 


\section{Graduation committee}

\section{Chair}

Prof.dr.ir. A. Veldkamp

University of Twente

\section{Promoters}

Prof.dr. A.K. Skidmore

Prof.dr. S. Jones

University of Twente

RMIT University

\section{Assistant promoters}

Dr. T. Wang

Members

Prof.dr. Z. Su

Prof.dr. W. Verhoef

Prof.dr. M. Herold

Prof.dr. R. Hill

University of Twente

University of Twente

University of Twente

Wageningen University

Bournemouth University 



\section{Acknowledgments}

This PhD journey has been an unforgettable period in my life. At this moment, I would like to express my sincere appreciation to all the people who have encouraged and assisted me in these years.

First and foremost, I would like to express my gratitude to my promoter, Prof. Andrew K. Skidmore. Andrew, thank you for your continuous guidance and support. Your immense knowledge about geoscience and ecology always enlighten my mind. During my most stressful days in the third year, it was your encouragement that led me regain my confidence. You taught me how to be a researcher as well as a mentor. Thank you for providing me the opportunity to join the double-badged $\mathrm{PhD}$ program, which greatly enriched my life.

I am deeply grateful to my co-promoter Dr. Tiejun Wang. Tiejun, thank you for introducing this fascinating topic about LiDAR to me. Thank you for your help whenever I came across with difficulties about research experiment, fieldwork logistics and personal career plan. You taught me how to keep on track and manage time more wisely. Without your dedicated supervision and prompt feedback, I cannot overcome many obstacles through my PhD study.

My sincere thanks go to my promoter in RMIT University, Prof. Simon Jones. Simon, thank you for your supervision over the course of my $\mathrm{PhD}$, and your support during my stay in Melbourne. Your expertise on remote sensing, especially in-situ observation, help me formulate many research ideas. I also appreciate your help with my integration in the RMIT community. Thanks to your support, my six months stay at Melbourne was both enjoyable and productive.

I acknowledge the Data Pool Initiative in Bavarian Forest National Park. 
In particular, I am deeply indebted to Dr. Marco Heurich, for his efforts in assisting fieldwork design, providing logistical facilities, as well as offering constructive research comments. I also thank Dr. Burkhard Beudert and Joe Premier for their help on data collection and scientific writing.

The support from many other people are essential in my $\mathrm{PhD}$ research. In particular, I would like to thank Xi, my colleague and friend, for your consistent technical and mental support through these years. I am also deeply grateful to Bryan, Haidi and Yifang for their help with fieldwork transportation and data collection. In addition, I appreciate the help from Elnaz, Tawanda, Roshanak, Tiejun and Anton. The fieldwork and workshop days we had at BFNP are truly lifetime treasures.

I am grateful to ITC for a nice working environment. In particular, I would like to thank Esther and Loes, for their tremendous help in all logistical aspects, for their cheerful and friendly spirit to make me feel warm. My sincere gratitude goes to Benno and Job for their help in poster and cover design, to Willem for his technical assistance, to Watse and Caroline for lab equipment assistance, to Prof. Andy Nelson for support at the NRS department. I am indebted to many NRS colleagues, Sugandh, Trini, Lucas, Abebe, Alby, Festus, Eddy, Thomas, Yousif, Iris, Henk, Louise, Michael, Panagiotis, Wieteke, Nina, Anna, and Marcelle, for their support.

I appreciate the friendship and help from many people in my $\mathrm{PhD}$ life at Enschede. My special thanks go to my office mates, i.e. Zhihui, Haili, Linlin, Bryan and Sam. I express my sincere gratitude to Xiaolong, $\mathrm{Xu}$, Xiaoling, Junping, Ruosha, Hong, Wen and Yijian for organizing many joyful parties and trips. I am also grateful to the following people for their support and friendship as well. They are: Fangyuan, Yiwen, Qiao, Xin, Weichao, Lilin, Peipei, Mijun, Mengna, Chengliang, Lianyu, Yunfei, Bob, Chrisitiaan, Xuelong, Donghai, Xiaojing, Yongming, Bingbing, Shaoning, Cesar, Mira, Behnaz, Bagher, Nastia, Egor, Georgios, Jan, HarmJan, Megan, Sammy, Yasser, Moiteela, Mengmeng, Fashuai, Zhenchao, Yifei, Peng, Andrea, Fernando, Lichun and Tina. My special thanks go to Tiejun's family, Wen and Li, for their delicious cooking which enable me to taste a flavor of home. My thanks also go to Eva Skidmore for her English editing which make my publishing more smooth. 
During my stay at Melbourne, I gained much support from many wonderful people. In particular, I would like to thank Daisy and Mahyat, for your company and caring. We had so many fun gatherings as well as unforgettable fieldwork in the Eucalypt forest. I would like to thank other people in RMIT who made me feel welcome as well. They are Bryan, Sam, Trung, Luke, Mariela, Lola, Karin, Ahmad, Chithrangani, Samuel, Salahuddin and John. My special thanks go to the HDR group in School of Science for their kind support with my $\mathrm{PhD}$ candidature.

Furthermore, I am deeply grateful to my formal supervisors in China, in particular Prof. Peijun Li and Dr. Yanlian Zhou. Thank you for your guidance in my academic path. Special thanks also go to my formal teachers, Prof. Weimin Ju, Dr. Shuhe Zhao, and Dr. Guang Zheng, for their advice and instructions. I am very grateful to my friends Mengwei, Qin, Ying, and Dan for their love and support.

Last but not least, my deepest gratitude goes to my parents. Dad and Mom, thank you for your unconditional love and caring. Thank you for your understanding and respect. Special thanks to my aunt and uncle who help taking care of my family in the difficulties. Special thanks go to my in-laws for their support. Finally, I would like to thank Peiqi, my beloved husband, for always standing by me and walking along this journey. Thank you for your patience, love, and support. 


\section{Contents}

Contents iv

1 Introduction 1

1 Importance of LAI and vertical LAI profile . . . . . . . . 2

2 Estimation of LAI and vertical LAI profile using remote sensing $\ldots \ldots \ldots \ldots \ldots \ldots \ldots \ldots \ldots \ldots \ldots \ldots$

3 Previous studies of LAI and vertical LAI profile estimation using LiDAR . . . . . . . . . . . . . . 5

4 Research questions and thesis structure . . . . . . . 11

2 Retrieving leaf angle distribution using terrestrial LiDAR 13

1 Introduction . . . . . . . . . . . . . . . . . . . 15

2 Research data . . . . . . . . . . . . . . . . 18

3 Method ................... 25

$4 \quad$ Results . . . . . . . . . . . . . . . . . 28

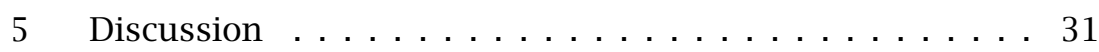

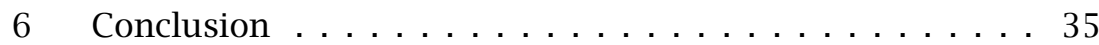

3 Variation of leaf angle distribution in natural beech forests 37

1 Introduction . . . . . . . . . . . . . . . . . . 39

2 Research data . . . . . . . . . . . . . . . . 42

3 Method ...................... . . 45

$4 \quad$ Results . . . . . . . . . . . . . . . . . . 50

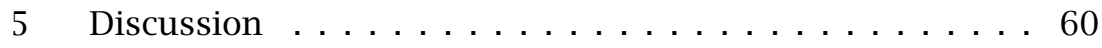

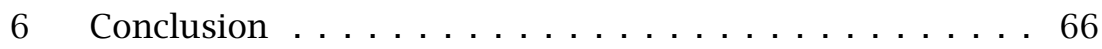

4 Scan angle impact on gap fraction estimation from airborne

$\begin{array}{ll}\text { LiDAR } & 69\end{array}$

1 Introduction $\ldots \ldots \ldots \ldots \ldots \ldots \ldots \ldots \ldots \ldots \ldots$ 
2 Materials and method . . . . . . . . . . 75

$3 \quad$ Results . . . . . . . . . . . . . . . . . 83

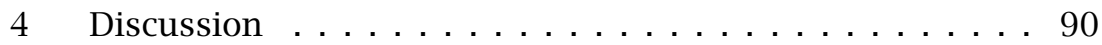

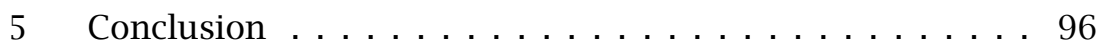

5 Topographic normalization effect on vertical LAI profile $\quad 97$

1 Introduction . . . . . . . . . . . . . . . . 99

2 Materials . . . . . . . . . . . . . . . . . 102

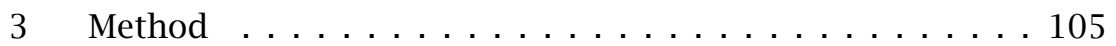

$4 \quad$ Results . . . . . . . . . . . . . . . . . . . . . . 109

5 Discussion and conclusion . . . . . . . . . . . . 114

6 Synthesis: Mapping the 3D distribution of leaves in forests $\begin{array}{ll}\text { using LiDAR } & 119\end{array}$

1 Summary . . . . . . . . . . . . . . . 120

2 Retrieving LAI and vertical LAI profile at local scale . . . . 121

3 Retrieving LAI and vertical LAI profile at regional scale . . 122

4 Retrieving LAI and vertical LAI profile at continental or global scale . . . . . . . . . . . . . . . . . . 129

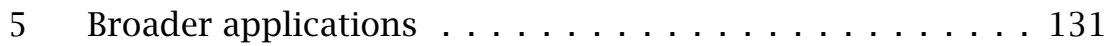

$\begin{array}{ll}\text { Bibliography } & 135\end{array}$

7 Summary 163

8 Samenvatting 165

9 Author's biography and publications 167 



\section{List of Figures}

1.1 Diagram of full waveform LiDAR and discrete return LiDAR (Lefsky et al., 2002) . . . . . . . . . . . . . . . . . . 4

1.2 Six commonly used leaf angle distribution types and the corresponding leaf projection function $\ldots \ldots \ldots \ldots \ldots$

1.3 Difference in the scanning patterns between airborne and terrestrial LiDAR $\ldots \ldots \ldots \ldots \ldots$

2.1 The distribution of European beech (Fagus sylvatica) and the location of the study area Bavarian Forest National Park in Europe . . . . . . . . . . . . . . . . . . . . . . 19

2.2 The distribution of the 36 European beech plots with both TLS and DHP data . . . . . . . . . . . . . . . . . . 19

2.3 Canopy height models of the 36 European beech plots of different structures . . . . . . . . . . . . . . . . . 20

2.4 Examples of a) c) e) g) virtual homogeneous stands with closed canopy and even canopy height, without any woody material; and b) d) f) h) virtual heterogeneous stands with discontinuous canopy and uneven tree spacing. . . . . . . . . . . . 23

2.5 Examples of the simulated DHP and simulated TLS point clouds in a) c) virtual homogeneous stands with closed canopy and even canopy height; b) d) virtual heterogeneous stands with discontinuous canopy and uneven tree spacing. . . . . . . 24

2.6 The flowchart to evaluate the consistency and accuracy of TLS and DHP in estimating leaf angle distribution (note: $\bar{\theta}$ is the average inclination angle) . . . . . . . . . . . . . . . 25

2.7 The average inclination angle $(\bar{\theta})$ estimated from TLS and from DHP using the field based dataset in the 36 European beech stands . . . . . . . . . . . . . . . . . . . . 29 
2.8 The LAD results accuracy from TLS and DHP in the synthetic dataset. a) the estimated average inclination angle $(\bar{\theta})$ from TLS; b) the estimated $\bar{\theta}$ from DHP . . . . . . . . . . . 30

2.9 The violation of path length assumption in DHP due to uneven tree height, uneven tree spacing and non-flat underneath topography in heterogeneous forests . . . . . . . . . 33

2.10 Inconsistency of the average inclination angle $(\bar{\theta})$ estimated from TLS and from DHP using the synthetic forest datasets . . 34

3.1 Six predefined mathematical functions used to approximate leaf angle distribution and the corresponding average leaf inclination angle $\left(\theta_{\text {mean }}\right) \ldots \ldots \ldots$. . . . . . . 39

3.2 European Beech (Fagus sylvatica) in the Bavarian Forest National Park. a) leaves; b) trunk and branch; c) a mature plot . . 43

3.3 The distribution of 36 European beech plots in the Bavarian Forest National Park . . . . . . . . . . . . . . . 43

3.4 Results of the leaf angle calculation accuracy using simulated dataset: a) d) the 3D models of two synthetic beech trees; b) e) the simulated TLS point clouds of the beech trees; c) f) true leaf angles and leaf angles estimated using the proposed method 50

3.5 Results of differentiating leaf and woody materials in part of a mature beech plot B32: a) before and b) after classification; detailed results at c) $8 \mathrm{~m}$ and d) $16 \mathrm{~m}$ above ground . . . . . 52

3.6 LAD at different canopy layers in the mature beech plot B01. a), b) and c) are the 3D distribution of leaf angles; d), e) and f) are the LAD histograms; g) summary of statistics . . . . . . . 54

3.7 LAD on plots of different successional status. The 3D distribution of leaf angles in a) a young plot B27; b) a medium plot B10; c) a mature plot B31; d) part of B27; e) part of B10; f) part of $\mathrm{B} 31 \ldots \ldots \ldots \ldots \ldots \ldots \ldots$

3.8 LAD of plots at different successional status: a) a young plot B27; b) a medium plot B10; c) a mature plot B31. . . . . . . 56

3.9 The correlation between plot median canopy height and plot a) average leaf inclination angle and b) skewness of leaf angle distribution (LAD) across all 36 European beech plots . . . . 57 
3.10 LAD variation at different canopy layers and across different stands, a) average leaf inclination angle; b) most frequent leaf inclination angle; c) skewness of LAD . . . . . . . . . . . . 58

3.11 a) The decreasing point density with height above ground in all 36 beech plots (point density was quantified by mean nearest neighbor points distance (MNNPD); b) leaf angle estimation accuracy decreases with decreasing point density from the simulated point clouds . . . . . . . . . . . . . . . 64

3.12 The value of leaf projection function $\mathrm{G}(\theta)$ under 6 predefined leaf angle distribution (LAD) assumptions and commonly used airborne LiDAR (ALS) scan angle range . . . . . . . . . . . . 65

4.1 Examples of a) European beech, b) Norway spruce, and c) mixed stands in the study area . . . . . . . . . . . . . . . 75

4.2 Airborne LiDAR flight lines and selected forest plots on the four transects (T1, T2, T3, T4) in the study area of Bavarian Forest National Park (DHP: digital hemispherical photograph) . 77

4.3 $P_{\text {gap }}$ of different angles measured from DHP at ground in deciduous, coniferous and mixed forest plots $\ldots \ldots \ldots .84$

4.4 Vertical $P_{\text {gap }}$ profiles derived from airborne LiDAR data of different scan angles in deciduous beech plots (red points: ground returns). Note: there is one giant coniferous tree in plot T4-23 . . . . . . . . . . . . . . . . . 85

4.5 Vertical $P_{\text {gap }}$ profiles derived from airborne LiDAR data of different scan angles in coniferous spruce plots (red points: ground returns) $\ldots \ldots \ldots \ldots \ldots \ldots$

4.6 Vertical $P_{\text {gap }}$ profiles derived from airborne LiDAR data of different scan angles in mixed plots (red points: ground returns) 87

4.7 Correlation between the $P_{\text {gap }}$ derived from DHP and the $P_{\text {gap }}$ derived from airborne LiDAR. a) without considering scan angle; b) scan angle ranges from $0^{\circ}-7^{\circ}$; c) scan angle ranges from $7^{\circ}-23^{\circ}$; d) scan angle exceeds $23^{\circ}$. In each sub figure, $\mathrm{N}$ is the number of plots $\ldots \ldots \ldots \ldots \ldots$ 
4.8 Correlation between nadir $P_{\text {gap }}$ and the difference of $P_{\text {gap }}$ measured from airborne LiDAR of different scan angles in deciduous, coniferous and mix forest plots. $\left(P_{\text {gap } 0-7}, P_{\text {gap } 7-23}\right.$ and $P_{\text {gap }>23}$ : gap fraction measured from LiDAR when scan angle ranges in $0^{\circ}-7^{\circ}, 7^{\circ}-23^{\circ}$, greater than $23^{\circ}$ respectively) . 89

4.9 Correlation between nadir $P_{\text {gap }}$ and the RMSD of each two vertical $P_{\text {gap }}$ profiles derived from airborne LiDAR of two different scan angles, in deciduous, coniferous and mix forest plots. ( $P_{\text {gap_prof0-7 }}, P_{\text {gap_prof7-23 }}$ and $P_{\text {gap_prof }>23}$ : vertical $P_{\text {gap }}$ profiles measured from LiDAR when scan angle ranges in $0^{\circ}-7^{\circ}$, $7^{\circ}-23^{\circ}$, greater than $23^{\circ}$ respectively) $\ldots \ldots \ldots \ldots$

4.10 Theoretical $P_{g a p}$ value change with zenith angle using predefined planophile, plagiophile, spherical and erectophile leaf angle distribution (de Wit 1965), when LAI $=1$ and $\lambda=1$ (ALIA: average leaf inclination angle) $\ldots \ldots \ldots \ldots . \ldots . \ldots 9$

5.1 Schematic illustration of how local topography affects the vertical leaf area distribution. Three plots (a), (b), and (c) with the same trees and leaf area but different spatial distribution of leaves on different local topography conditions. Differences in the vertical structure of the three plots are eliminated after topographic normalization . . . . . . . . . . . . 101

5.2 Study area (Bavarian Forest National Park) location in Germany and the location of the 33 selected plots . . . . . . . . 103

5.3 Comparison between the vertical LAI profiles with and without topographic normalization (bar: raw LAI profile; curve: smoothed LAI profile). . . . . . . . . . . . . . . . . . 110

5.4 Comparison between canopy layers detected from vertical LAI profiles with (in green) and without (in blue) topographic normalization . . . . . . . . . . . . . . . . 111

5.5 (a) Relationship between the decrease of vertical extent and mean slope; (b) Relationship between the decrease of vertical extent and standard deviation of slope . . . . . . . . . . . 113

5.6 Relationship between a) canopy layer number decrease and mean slope, b)canopy layer number decrease and standard deviation of slope, c) entropy decrease and mean slope, d) entropy decrease and standard deviation of slope . . . . . . 114 
5.7 Comparison between point clouds of plot T3-28 with and without topographic normalization, respectively. (Same viewing angle and azimuth angle, red points are detected ground returns) . . . . . . . . . . . . . . . . . . . . . . . . . . . 115

5.8 Illustration of different decrease of vertical LAI/PAI profile complexity after topographic normalization, even with the same local topography and same vegetation. The differences are caused by the different spatial distribution of trees. (dashed line: estimated canopy layer location) . . . . . . . . . . . . 116

6.1 a) terrestrial LiDAR (TLS) estimated the average inclination angle $\bar{\theta}$ much more accurately than b) digital hemispherical photography (DHP) in the synthetic dataset . . . . . . . . 122

6.2 The LAI map retrieved from airborne LiDAR data in the Bavarian Forest National Park in 2017 . . . . . . . . . . . . 123

6.3 Consistency of LAI estimated from airborne LiDAR and DHP . 124

6.4 The vertical LAI profile map retrieved from airborne LiDAR in the Bavarian Forest National Park in 2017 . . . . . . . . . 125

6.5 Two forest areas with similar LAI ranges but distinct vertical LAI distribution pattern revealed by airborne LiDAR (for legend please refer to Fig 6.2 and 6.4) . . . . . . . . . . . 126

6.6 The coverage of GLAS data in California from 2003-2007 (Tang et al., 2014b) . . . . . . . . . . . . . . . . . . . . . . 129

6.7 The footprint size and spacing of GEDI (Dubayah et al., 2014) 130 



\section{List of Tables}

2.1 Basic information of the 36 European beech plots . . . . . . 21

2.2 Summary of the LAD results estimated from TLS and DHP in the field based dataset of 36 European beech stands . . . . . 29

2.3 Summary of the LAD results estimated from TLS and DHP in the synthetic dataset of 24 virtual stands . . . . . . . . 31

2.4 Comparison of TLS and DHP in measuring leaf angle distribution $(\mathrm{LAD}) \ldots \ldots \ldots \ldots$. . . . . . . . . . . . . . . . 35

3.1 Basic information of the 36 European beech plots . . . . . . 44

3.2 Radiometric and geometric features used to differentiate leaf

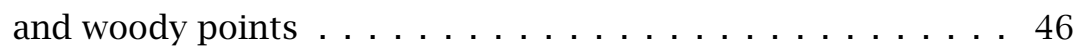

3.3 The overall accuracy (OA) of classification between leaf and woody materials in the 36 plots $\ldots \ldots \ldots \ldots$. . . . 51

3.4 Summary of LAD statistics across three different stands . . . 56

3.5 LAD results on different canopy layers and across all plots . . 59

3.6 Suggested choice of predefined functions for LAD approximation in European Beech stands . . . . . . . . . . . . . . 61

4.1 Basic information of the 30 Bioklim plots with DHP measure-

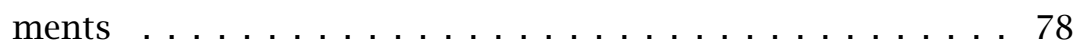

4.2 Paired-sample $t$-test between $P_{\text {gap }}$ derived from LiDAR data of different scan angles . . . . . . . . . . . . . 84

5.1 Basic information of the 33 plots in the study area $\ldots . . .104$

5.2 The vertical complexity of canopy structure derived from the vertical LAI profiles with (TN) and without topographic normalization (noTN) and their differences (diff) . . . . . . . . 112

5.3 Summary of the two sample $t$-test statistics for canopy vertical complexity metrics with and without topographic normalization 113 



\section{Introduction}




\section{Importance of LAI and vertical LAI profile}

Forests cover about 30\% of the Earth land surface (Hansen et al., 2013). They provide numerous ecological services, including carbon storage, water supply, climate regulation, and habitat for various species. From 1990 to 2007 , forests sequestered up to $60 \%$ of the anthropogenic carbon emissions (Pan et al., 2011). Human well-being strongly depends on the status of forests. However, Earth is undergoing accelerating tropical forest loss (Luo et al., 2013) and temperate forest mortality (Senf et al., 2018). Mapping and monitoring forests is extremely important.

Leaves are the interface between forests and atmosphere, the place where most of the energy fluxes exchange. The amount and distribution of leaves determine the radiation interception (Verhoef, 1984), carbon sequestration (Barr et al., 2004), precipitation interception and evapotranspiration (Wilson et al., 2001) in forests. Therefore, leaf area index (LAI), defined as one half the total green leaf area per unit ground surface area (Chen et al. 1997), has been widely used as a key component to model ecosystem productivity, hydrological process and climate change (Weiss et al., 2004; Van der Tol et al., 2009; Zheng and Moskal, 2009). LAI has been identified as both an essential climate variable (ECV) (Baret et al., 2013; Bojinski et al., 2014), and an essential biodiversity variable (EBV) (Skidmore and Pettorelli, 2015).

The vertical distribution of leaves warrants particular importance for forest biodiversity. Many species including birds (Walther, 2002; Siegel and DeSante, 2003), primates (Davies et al., 2017), arthropods (Halaj et al., 2000), and reptiles (Shine et al., 2002) were reported to have structural preferences in forests. The vertical LAI profile, which is the vertical distribution of leaf area expressed as a function of height, has been widely used in wildlife habitat modelling (MacArthur and MacArthur, 1961; Goetz et al., 2007; Muller et al., 2013). Compared with LAI, the vertical LAI profile is a more realistic representation of the 3D canopy structure. It has been used to map forest succession stages (Falkowski et al., 2009; Marselis et al., 2018), canopy strata (Wilkes et al., 2015a) and forest vertical structure complexity (Stark et al., 2012; Atkins et al., 2018). 


\section{Estimation of LAI and vertical LAI profile using re- mote sensing}

The most accurate LAI and vertical LAI profile values are acquired by direct leaf collection and leaf area measurement using either planimetric or gravimetric methods (Tang 2015). However, these methods are costly, time-consuming and sometimes not feasible for forests (Bréda, 2003). Remote sensing techniques provide a non-destructive, rapid and economical way for estimating LAI across a wide range of spatial and temporal scales.

\subsection{Passive remote sensing of LAI and vertical LAI profile and its limitation}

At local scales, remote sensing based indirect techniques have become the primary in-situ LAI methods, such as LAI-2000 or LAI-2200 (LiCOR, 2009), digital hemispherical photography (DHP) and TRAC (Jonckheere et al., 2004). They have been intensively used to collect ground LAI reference for validating satellite products. At larger spatial extent, passive optical sensors such as MODIS (Myneni et al., 2002), SPOT (Weiss et al., 2007), and MERIS (Bacour et al., 2006) are essential to generate spatially explicit LAI. Several global LAI products are available, including GLASS (Zhao et al., 2013), GEOV1 (Baret et al., 2013), GLOBMAP (Liu et al., 2012), and MODIS (C5, C6) (Yan et al., 2016).

However, there are some limitations with passive remote sensing in mapping LAI. Previous studies have revealed large inconsistency and uncertainty among several LAI products at regional to global scales from passive sensors (Fang et al., 2013; Jiang et al., 2017). Part of the uncertainty was attributed to the saturation of spectral signals over high LAI areas (Liu et al., 2018b). For instance, LAI estimated from the normalized difference vegetation index (NDVI) using passive remote sensing is known to saturate at values around 3 to 4 , which is below the global mean LAI value of 4.7 in tropical forests (Asner et al., 2003). While the saturation problem has long been discovered, it cannot be easily solved using passive remote sensing only. Furthermore, retrieving the vertical LAI profile is beyond the capabilities of passive sensors. 


\subsection{Potential of LiDAR for LAI and vertical LAI profile estimation}

In the past decades, LiDAR (Light Detection and Ranging) has emerged as a new active remote sensing technology. Time-of-flight LiDAR measures the distance between a sensor and a target based on half the elapsed time between the emission of a pulse and the detection of a reflected return (Baltsavias, 1999). It can be classified as either discrete return or full waveform types (Fig 1.1). Full waveform LiDAR digitizes the entire backscattered energy, while discrete return LiDAR records single or multiple returns from a return pulse (Wulder et al., 2012). The ability to capture precise 3D information has made LiDAR a powerful tool in estimating various forest structure metrics (e.g. canopy height and aboveground biomass) with unprecedented accuracy (Drake et al., 2002; Lefsky et al., 2002).

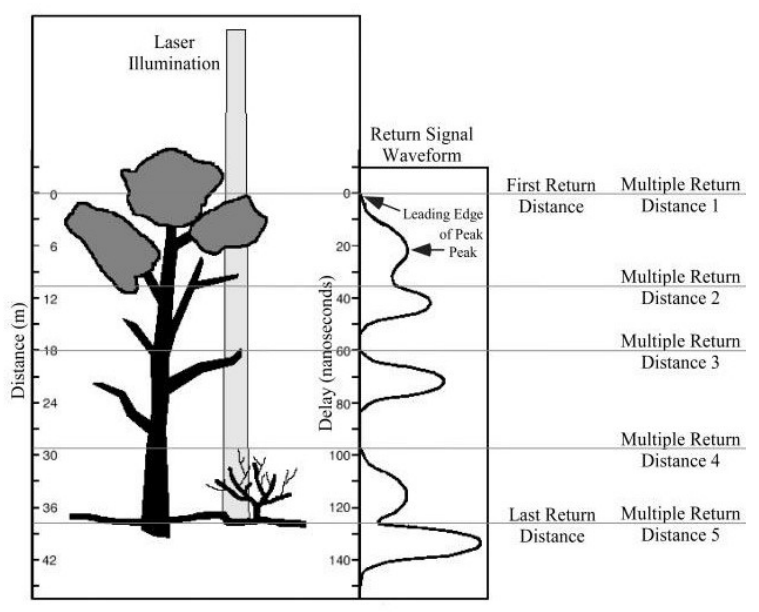

Figure 1.1: Diagram of full waveform LiDAR and discrete return LiDAR (Lefsky et al., 2002)

Compared to passive remote sensing, LiDAR has several advantages, which offers great potential to render itself as a superior tool for mapping LAI and the vertical LAI profiles (Zhao and Popescu, 2009). First, LiDAR can penetrate the canopy and detect understory vegetation (Hill and Broughton, 2009). This penetrating ability allows for alleviation of the saturation problem in passive sensors, and better characterization of vegetation distribution beneath the canopy surface. Second, with the 
precise 3D information, LiDAR can be used to retrieve the vertical LAI profile, which can not be accomplished with conventional passive sensors like MODIS or Landsat.

\section{Previous studies of LAI and vertical LAI profile estim- ation using LiDAR}

\subsection{Theoretical Background}

\subsubsection{Definition of LAI and vertical LAI profile}

Various definitions of LAI were proposed for different applications. Two most frequently used definitions are "true LAI" and "effective LAI". True LAI was defined as one half the total green leaf area per unit ground surface area (Chen et al., 1997). Effective LAI was defined as one half of the total area of light intercepted by leaves per unit ground surface area (Chen and Black, 2010). Effective LAI differs from true LAI in that it does not account for the non-random distribution of foliage in the canopy.

Another important term is the "plant area index (PAI)". Many studies and products use the term LAI but actually refer to PAI, since the distinction between photosynthetic and non-photosynthetic elements was not made (Weiss et al., 2004). Due to the interchangeable usage of PAI and LAI in the literature, this thesis adopts the term LAI, but the effect of woody elements will be discussed.

The vertical LAI profile is the vertical distribution of leaf area expressed as a function of height. It was also referred to as foliage height profile (MacArthur and Horn, 1969), vertical foliage profile (Tang et al., 2014b), vertical leaf area density profile (Lin and West, 2016), vertical plant profile (Calders et al., 2014) and canopy height profile (Harding et al., 2001; Lefsky et al., 2002; Hilker et al., 2010) in previous studies.

\subsubsection{Gap fraction model}

The gap fraction model based on the Beer-Lambert law is the fundamental component used in most physically based LAI retrievals. Its basic 
principle is that "the attenuation of radiation passing though a canopy is linked with the amount and distribution of leaves in the canopy, through mathematical models". In the case of a random spatial distribution of infinitely small leaves,

$$
P_{\text {gap }}(\theta, \varphi)=e^{-G(\theta, \varphi) L / \cos (\theta)}
$$

where $\theta$ is the inclination angle and $\varphi$ is the azimuth angle of the light beam penetrating the canopy. $P_{g a p}(\theta, \varphi)$ is named gap fraction, defined as the probability of a light beam to directly pass through the canopy in the direction $(\theta, \varphi)$ without any collision(Armston et al., 2013). $L$ is the LAI, and $G(\theta, \varphi)$ is the leaf projection function, which is the mean projection of unit foliage area in the direction $(\theta, \varphi)$ (Nilson, 1971; Weiss et al., 2004). Even in canopies with non-random distributed leaves, an extended model has been proposed,

$$
P_{\text {gap }}(\theta, \varphi)=e^{-G(\theta, \varphi) \lambda L / \cos (\theta)}
$$

where $\lambda$ is the clumping index, depending on the canopy structure. $\lambda$ is smaller than 1 for aggregated canopies, while greater than 1 for regular dispersed canopies. The effective LAI $\left(L_{\text {eff }}\right)$ is the product of clumping index and true LAI,

$$
L_{\mathrm{eff}}=\lambda L
$$

From Eq 1.1, 1.2 and 1.3, LAI can be retrieved from,

$$
L_{\text {eff }}=\frac{-\ln \left(P_{\text {gap }}(\theta, \varphi)\right) \cos (\theta)}{G(\theta, \varphi)}
$$

In terms of vertical LAI profile,

$$
\begin{aligned}
& L_{\text {cum }}(h)=\frac{-\ln \left(P_{\text {gap }}(h, \theta, \varphi)\right) \cos (\theta)}{G(h, \theta, \varphi)} \\
& l(h)=\frac{\partial L_{\text {cum }}(h)}{\partial h}
\end{aligned}
$$

where $L_{\text {cum }}(h)$ is the cumulative LAI profile as a function of height. Its derivative, i.e. $l(h)$ is the vertical LAI profile or leaf area density profile, defined as the the leaf area per unit volume at level $h$ in the canopy. 
Based on Eq 1.4, 1.5 and 1.6, it is clear that LAI and vertical LAI profiles are retrieved from gap fraction and leaf projection function values. The leaf projection function $G(\theta, \varphi)$ is determined by how the leaves are oriented inside the canopy (Wang et al., 2007),

$$
\begin{array}{r}
G(\theta, \varphi)=\frac{1}{2 \pi} \int_{0}^{2 \pi} \int_{0}^{\pi / 2}\left|\cos \theta \cos \theta_{1}+\sin \theta \sin \theta_{1} \cos \left(\varphi-\varphi_{1}\right)\right| g\left(\theta_{1}, \varphi_{1}\right) \\
\times \sin \theta_{1} d \theta_{1} d \varphi_{1}
\end{array}
$$

where $\theta_{1}$ is the zenith angle of a leaf normal vector (inclination angle), and $\varphi_{1}$ is the azimuth angle of a leaf. $g\left(\theta_{1}, \varphi_{1}\right)$ is the leaf angle distribution (LAD), defined as the probability of leaf area with the leaf inclination angle between $\theta_{1}$ and $\theta_{1}+d \theta_{1}$ and the leaf azimuth angle between $\varphi_{1}$ and $\varphi_{1}+d \varphi_{1}$ (Weiss et al., 2004). Both categorical and continuous functions have been proposed to model the LAD. Six commonly used categorical LAD types are shown in Fig 1.2, where the planophile canopy is dominated by horizontally growing leaves while the erectophile canopy is dominated by erectly inclined leaves. At the convergent angle of $57.5^{\circ}$, the $G(\theta, \varphi)$ approaches 0.5 regardless of the LAD type.
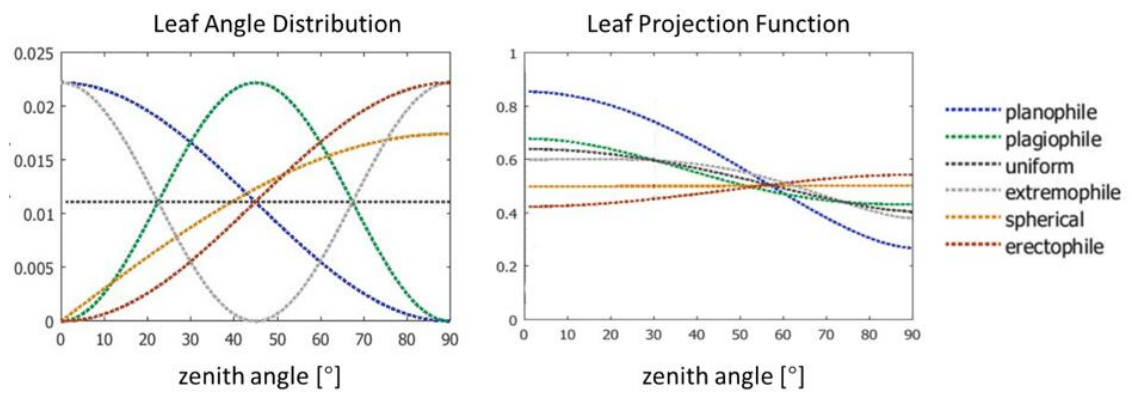

Figure 1.2: Six commonly used leaf angle distribution types and the corresponding leaf projection function

To sum up, the gap fraction model is the fundamental method used in this study to retrieve LAI and the vertical LAI profile. The clumping effect and woody elements were not corrected in this study, but will be investigated in future research. From Eq 1.4 to 1.7, it is demonstrated that an accurate estimate of gap fraction $\left(P_{\text {gap }}(\theta, \varphi)\right)$ and leaf angle distribution (LAD, $\left.g\left(\theta_{1}, \varphi_{1}\right)\right)$ are prerequisite for the final LAI results. 
The other two parameters $\theta$ and $h$ may also affect the vertical LAI profile result.

\subsection{Previous studies of LAI and vertical LAI profile retrieval using LiDAR}

At local scale, terrestrial LiDAR has been used to estimate LAI and vertical LAI profile (Lovell et al., 2003; Jupp et al., 2009; Zhao et al., 2011), serving as the validation for airborne and satellite products (Tang et al., 2014a). Compared to conventional techniques such as DHP or LAI-2000, terrestrial LiDAR is insensitive to illumination condition (Calders et al., 2018). Moreover, the differentiation of foliage and woody elements is feasible, thus enabling correction of woody elements and extraction of true LAI (Zhu et al., 2018b). Another advantage of terrestrial LiDAR is its capability to estimate LAI for individual tree (Hu et al., 2018), which is impossible for DHP or LAI-2000.

At regional scale, airborne LiDAR enables wall-to-wall mapping of LAI and vertical LAI profile. Three retrieval methods have been proposed including the empirical model (Alonzo et al., 2015), the gap fraction model (Korhonen et al., 2011), and the radiative transfer model (Koetz et al., 2006). Empirical models rely on regression of in-situ LAI values with a set of LiDAR metrics (Jensen et al., 2008). They tend to be applicable for a single species in a single geographical area. In heterogeneous mixed forests, the gap fraction model as introduced in section 3.1.2 exhibited higher accuracy (Richardson et al., 2009). Gap fraction is approximated by various laser penetration metrics (Morsdorf et al., 2006; Solberg et al., 2009) from discrete return LiDAR or estimated from backscattered energy using full waveform LiDAR (Armston et al., 2013; Tang et al., 2012). Small footprint and high pulse density even leads to the retrieval of LAI and vertical LAI profile for individual tree (Oshio et al., 2015). A combination of leaf-on and leaf-off airborne LiDAR flights facilitates the correction of woody element effects to retrieve true LAI for deciduous trees (Zhu et al., 2019 in preparation).

At continental or global scale, spaceborne LiDAR is the unique dataset to provide large area vertical LAI profile product. LAI and vertical LAI profile (Tang et al., 2014b) has been retrieved from the Geoscience Laser Alti- 
meter System (GLAS), a large footprint ( $\sim 65 \mathrm{~m}$ in diameter) full waveform profiling LiDAR from 2003 to 2009. The method was extended to map the vertical LAI profile across the United States (Tang et al., 2016) and Amazon (Tang and Dubayah, 2017). Very recently on Dec 5 2018, Global Ecosystem Dynamics Investigation (GEDI), a new spaceborne LiDAR with medium footprint ( $\sim 25 \mathrm{~m}$ in diameter) was successfully launched. Its LAI and vertical LAI profile product will bring numerous opportunities for ecological and biodiversity studies (Dubayah et al., 2014; Skidmore and Pettorelli, 2015).

\subsection{Unresolved challenges at different scales}

The retrieval of LAI and vertical LAI profile faces several challenges at different scales. According to Eq 1.4 to 1.7, the gap fraction and LAD are prerequisite for LAI and vertical LAI profile retrieval, while $\theta$, and $h$ may also affect the results. In the following, challenges related to these factors will be introduced.

At local scale, which in-situ LAI technique is the most accurate and reliable still remains unknown. In-situ measurement is the basis for validation of airborne or satellite products. However, large LAI inconsistency and uncertainty were revealed among different in-situ techniques including DHP, LAI-2000, and terrestrial LiDAR (Garrigues et al., 2008; Woodgate et al., 2015). Part of the inconsistency arise from the estimation of gap fraction (Garrigues et al., 2008; Woodgate et al., 2015). Terrestrial LiDAR was suggested to be preferential than other techniques (Calders et al., 2018). Nevertheless, the consistency and accuracy in the estimation of LAD, another key factor in LAI and vertical LAI profile retrieval, remains unexplored. If terrestrial LiDAR has more accurate LAD estimate, it is potentially giving more accurate LAI estimate.

At regional scale, the variation of LAD in natural forests has rarely been explored. Using airborne LiDAR, the importance of LAD estimate for LAI mapping becomes more pronounced. Unlike terrestrial LiDAR which measures the canopy from below across a large zenith angle range (for example $\left[-40^{\circ}, 90^{\circ}\right]$ with rotation tilt), airborne LiDAR measures the canopy from above, with much smaller zenith angle ranges (for example $\left.\left[-30^{\circ}, 30^{\circ}\right]\right)$ as shown in Fig 1.3. One cannot use the convergent angle 
of $57.5^{\circ}$ when the leaf projection function can be approximated as 0.5 regardless of the LAD type (Fig 1.2). At $0^{\circ}-30^{\circ}$, different LAD lead to quite large difference of leaf projection function values. Inappropriate estimation of LAD was reported to result in $47 \%$ underestimation of LAI (Pisek et al., 2013). In most current studies, LAD was assumed as the spherical distribution. Is this a valid assumption in natural forests? Is terrestrial LiDAR able to capture the variation of LAD? This remains poorly understood.
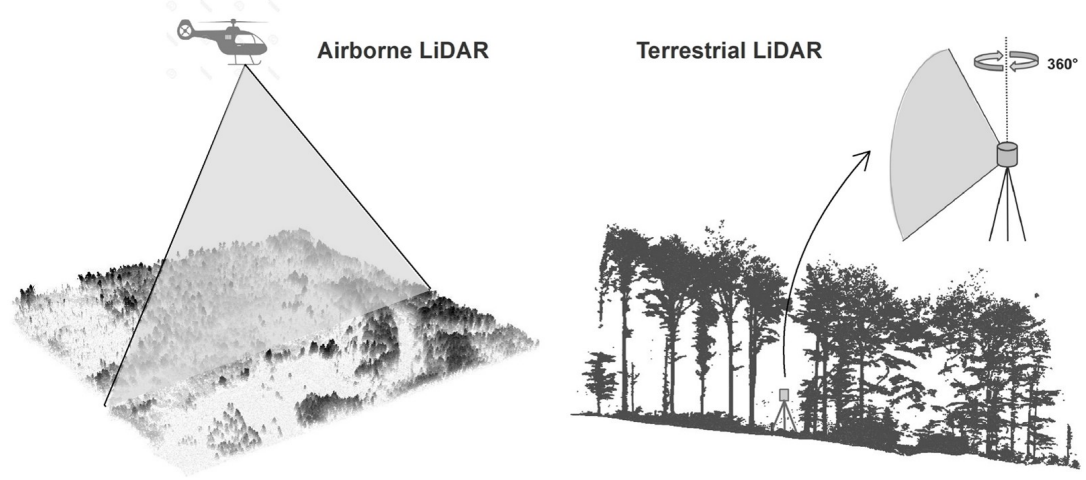

Figure 1.3: Difference in the scanning patterns between airborne and terrestrial LiDAR

At regional scale, the LAI uncertainty caused by airborne LiDAR flight settings (for example, scan angle) has rarely been investigated. As demonstrated in section 3.1.2, accurate measurement of $P_{\text {gap }}$ and vertical $P_{\text {gap }}$ profile is critical for subsequent LAI and vertical LAI profile estimation. In many previous studies, the scan angle of airborne LiDAR was overlooked and a nadir direction was assumed (Hilker et al., 2010; Tang et al., 2012). This may not be a big problem in earlier studies, when the scan angle had a smaller range $\left(\sim 10^{\circ}\right)$ close to nadir (Riaño et al., 2004; Solberg et al., 2006). However, in recent years, researchers have used airborne LiDAR data with a much larger scan angle range $\left( \pm 30^{\circ}- \pm 45^{\circ}\right)$ in forests (Fieber et al., 2015; Cao et al., 2016; Marino et al., 2016; Gu et al., 2018). Using multi temporal flights, it is even more difficult to maintain exactly the same scan angle. It is known to us that the precision of canopy height estimates decreased with increasing off-nadir scan angle (Lovell et al., 2005; Pang et al., 2011). Accordingly, it is necessary to investigate the scan angle impact on $P_{\text {gap }}$ and vertical $P_{\text {gap }}$ profile. 
In the retrieval of vertical LAI profile, the un-even topography is an additional factor to consider. The vertical LAI profile is a function of height $h$. But this $h$ could refer to local height or sea level height. In most studies, a topographic normalization algorithm was applied to retrieve local height. Through this step, all vegetation elements were put onto a flat terrain. However, for montane forests with un-even topography, this preprocessing may severely change the 3D topology of vegetation. Especially many derivative metrics from the vertical LAI profile such as canopy layer counts and the vertical complexity are all dependent on $h$. The effect of topographic normalization on the vertical LAI profile was never evaluated.

\section{Research questions and thesis structure}

Accurate estimation of LAI and vertical LAI profile is the basis for its further application in climate modelling or biodiversty modelling. The aim of this research is to improve the retrieval of LAI and vertical LAI profile at plot and regional scale, using terrestrial and airborne LiDAR.

Specifically, four research questions are outlined below:

1. Which in-situ technique measures the leaf angle distribution (LAD) more accurately? Terrestrial LiDAR or digital hemispherical photography (DHP)?

2. Is the spherical LAD a valid assumption in natural beech forests? How much is the LAD variation?

3. What is the effect of off-nadir scan angle in gap fraction and vertical gap fraction profile retrieval using airborne LiDAR?

4. What is the effect of topographic normalization in vertical LAI profile retrieval using airborne LiDAR?

This thesis consists of six chapters. Chapter 1 presents the research background, identify the existing challenges and propose specific research questions in this $\mathrm{PhD}$ thesis. Chapter 2 to 5 address the above-mentioned four specific objectives. Chapter 6 concludes the thesis with a summary of significant findings in the thesis, followed by a discussion of mapping the 3D distribution of leaves in forests at multiple scales. The broader 
1. Introduction

application of this thesis in forest ecology research is outlined. Three peer reviewed ISI journals have been published, and another one is to be submitted. 


\section{Retrieving leaf angle distribution using terrestrial LiDAR *}

*This chapter is based on: Liu, J., Wang, T., Skidmore, A., Jones, S., Heurich, M., 2018. Comparing terrestrial LiDAR and digital hemispherical photography in estimating leaf angle distribution in broadleaf forests. (to be submitted) 


\begin{abstract}
Leaf inclination plays a crucial role in regulating the radiation, carbon and water fluxes in plant canopies. Accurate measurement of its distribution function, i.e., the leaf angle distribution (LAD), is very important for modelling photosynthesis as well as estimating leaf area index (LAI). In spite of its importance, LAD is one of the most poorly constrained model parameters due to measurement challenges. Both digital hemispherical photography (DHP) and terrestrial LiDAR (TLS) have been used to measure in-situ LAD. However, the results consistency and relative accuracy from these two techniques have never been evaluated.

In this research, we aim to evaluate which in-situ technique, either DHP or TLS, measures LAD more accurately. Both filed based and synthetic datasets are used. The field based datasets are collected from 36 natural European beech stands covering a range of forest structures. The synthetic datasets are generated from 24 virtual forests through TLS and DHP simulators. Afterwards, the LAD is retrieved from TLS point clouds using a geometrical method, and from DHP using a gap fraction inversion method respectively. Results from the field based datasets show a significant difference and inconsistency $(r=0.19, p=0.26)$ between the average inclination angle $(\bar{\theta})$ retrieved from TLS $\left(\bar{\theta} \in\left(44^{\circ}, 53^{\circ}\right)\right)$ and DHP $\left(\bar{\theta} \in\left(18^{\circ}, 76^{\circ}\right)\right)$. Results from the synthetic datasets show that the accuracy of $\bar{\theta}$ from TLS $\left(R^{2}=0.79\right.$, RMSE $\left.=6.41^{\circ}\right)$ is considerably higher than that obtained from DHP $\left(R^{2}=0.12\right.$; RMSE $\left.=16.40^{\circ}\right)$.

This study demonstrates that the LAD estimated from TLS and DHP does not tally. Based on the synthetic dataset, TLS estimates leaf inclination more accurately than DHP. We therefore recommend TLS to serve as the ground-based technique in measuring leaf inclination in broadleaf forests.
\end{abstract}




\section{Introduction}

Leaf inclination is an important component of canopy structure. How the leaves incline and orient inside a canopy strongly regulates the radiation interception (Weiss et al., 2004; Niinemets, 2010), biomass production (Sakamoto et al., 2006), rainfall interception (Crockford and Richardson, 2010), and evapotranspiration (King, 1997). For some species, leaf inclination can serve as an indicator of stress. Drought-stressed soybean plants are shown to have steeper inclined leaves than well-watered soybean plants (Biskup et al., 2007). Leaf inclination may also reveal an evolutionary strategy of plants adapting to biotic and abiotic factors (Norman and Campbell, 1989). For example, a latitude cline was found for Arabidopsis thaliana, which has more erect leaves in lower latitudes to maximize photosynthesis (Hopkins et al., 2008). From a remote sensing perspective, leaf inclination is the primary control on nadir viewed canopy reflectance (Asner, 1998).

For a broadleaf, leaf inclination angle $(\theta)$ is the angle between the zenith direction and the leaf normal vector. The leaf angle distribution (LAD), or leaf inclination distribution function (LIDF), of canopies refers to the probability of all leaves inclined at different directions. The average inclination angle $(\bar{\theta})$ is the mean value of inclination angles of all leaves. LAD is widely used in modeling vegetation processes, such as radiative transfer (Verhoef, 1984), photosynthesis (Van der Tol et al., 2009; Yang et al., 2017), and rainfall interception (Xiao et al., 2000). LAD is also a prerequisite in estimating leaf area index (LAI) (Weiss et al., 2004) and vertical LAI profile. Due to measurement difficulty, LAD is often simplified as predefined mathematical functions (de Wit, 1965). For instance, a canopy of planophile LAD is dominated by horizontal leaves ( $\bar{\theta}$ $=26.8^{\circ}$ ), while a canopy of erectophile LAD is dominated by erect leaves $\left(\left(\bar{\theta}=63.2^{\circ}\right)\right.$ (Lemeur and Blad, 1975). Over-simplification of LAD has made it one of the most poorly constrained model parameters (Ollinger, 2011). A previous research has demonstrated up to $47 \%$ underestimation of LAI when a false LAD is assumed (Pisek et al., 2013).

Airborne and satellite remote sensing data have been used to estimate LAD through reflectance inversion (Jacquemoud et al., 2000; Houborg et al., 2007; Atzberger and Richter, 2012; Bayat et al., 2018; Ferreira et al., 
2018). However, none of these studies assessed the accuracy of LAD results, mainly due to the difficulty in acquiring in-situ reference value of LAD. At ground level, the geometric measurement of individual leaves is a commonly used approach to measure LAD. The inclination of each leaf can be manually measured using inclinometer (Ross, 1981), point quadrat (Wilson, 1960), or spatial coordinate apparatus (Lang, 1973). Nevertheless, manual methods easily disturb the canopy and demand much time as well. At least 75 leaves per tree should be measured across the vertical tree profile to obtain reliable estimates of the LAD (Pisek et al., 2013). The leveled digital canopy photography is a non-contact approach, where photographs are taken around the canopy. Individual leaves are visually inspected from each photo and thus LAD is calculated through image processing (Pisek et al., 2011). However, this method is difficult to implement in natural forests since it is very challenging to take pictures of leaves higher than $2 \mathrm{~m}$.

Recently, terrestrial LiDAR (TLS) has revolutionized the way we measure ecosystem structure (Danson et al., 2018). TLS can rapidly generate highdensity point clouds of vegetation canopies. With small beam divergence and high pulse frequency, individual leaf can be delineated (Hosoi and Omasa, 2012; Zhu et al., 2017). Separation of leaf and woody elements is also possible using TLS point clouds (Zhu et al., 2018a; Li et al., 2018c; Ferrara et al., 2018). LAD is then retrieved from TLS by reconstructing leaf surfaces and calculating normal vectors (Zheng and Moskal, 2012; Bailey and Mahaffee, 2017; Li et al., 2018a).

Radiation inversion is another approach to estimate LAD (Chen et al., 1991; Wagner and Hagemeier, 2006; Garrigues et al., 2008; Macfarlane et al., 2014). This method does not generate data on individual leaf scale. Instead, only the plot-averaged measure, i.e., the average inclination angle $(\bar{\theta})$, can be retrieved (Biskup et al., 2007). The underlining principle is that the average inclination angle $(\bar{\theta})$ is related to radiation attenuation through mathematical models. Therefore, it is possible to measure the radiation attenuation and then inverse the measurements to estimate $\bar{\theta}$ (Chen et al., 1991; Weiss et al., 2004). Both LAI-2000 (LiCOR, 2009) and the digital hemispherical photography (DHP) estimate LAD based on this theory. Due to inability to differ leaf and woody materials, the result is more precisely to be the average plant inclination angle, rather than the 
average leaf inclination angle (Chen et al., 1991).

Although both TLS and DHP have been used to measure LAD in ground surveys, there is no research on comparing these two techniques, and evaluating their accuracy. The main challenge lies in the acquisition of $100 \%$ true LAD reference values. Unlike metrics such as LAI or canopy height, of which the reference value may be acquired through destructive sampling, the true value of $\mathrm{LAD}$ is almost impossible to achieve in natural environment. Since it is almost impossible to measure all leaves without disturbing the orientation of other leaves.

Previous studies investigated the consistency of TLS and DHP in estimating gap fraction (Woodgate et al., 2015) and LAI (Calders et al., 2018) in various forest systems. However, no studies have evaluated the retrieval of LAD. TLS and DHP are two techniques intrinsically based on different theories and sensors independent of each other. If the LAD results from them are not consistent, then their relative accuracy evaluation is required. In this regard, synthetic dataset offers an alternative avenue to provide $100 \%$ true $\mathrm{LAD}$ reference values for evaluating the two techniques. A few recent studies used synthetic DHP in evaluating LAI retrieval (Leblanc and Fournier, 2014; Zou et al., 2018). Other studies utilized simulated LiDAR point clouds in evaluating the accuracy of tree height (Hämmerle et al., 2017), light interception (Perez et al., 2018), LAI (Liu et al., 2017b; Chen et al., 2018), and LAD (Liu et al., 2019). To the best of our knowledge, no studies have been carried out to assess the accuracy of TLS and DHP in LAD measurement.

In this research, we aim to evaluate the consistency and accuracy of the leaf angle distribution (LAD) retrieved from TLS and DHP. Specifically we set out to investigate the topic from two aspects. First, field based datasets collected in natural broadleaf forests are used to evaluate the consistency of the average inclination angle $(\bar{\theta})$ from TLS and DHP. Second, synthetic datasets generated from virtual forests through LiDAR and DHP simulators are used to evaluate the absolute accuracy of $\bar{\theta}$. 


\section{Research data}

\subsection{Field based dataset}

\subsubsection{Study area and beech plots}

The study area is the Bavarian Forest National Park in southeastern Germany. It is a low mountain range natural forest ecosystem in Central Europe (Fig. 2.1). The elevation ranges from $650 \mathrm{~m}$ to $1453 \mathrm{~m}$, generally with gentle slope gradients (Heurich et al., 2010). In order to protect biodiversity and the natural dynamic processes, the park is managed by a non-intervention strategy. This has led to a variety of forest structures in the study area (Heurich et al., 2011). The dominant tree species in the study area are Norway spruce (Picea abies) (67\%) and European Beech (Fagus sylvatica) (24.5\%) (Cailleret et al., 2014). European beech stands are chosen as the plant type under study due to its broadleaf feature. European beech is one of the most important and widespread broadleaf trees in Europe (San-Miguel-Ayanz et al., 2016) (Fig 2.1). The beech leaves are elliptical. Leaf size ranges in $25-40 \mathrm{~cm}^{2}$, with $5-10 \mathrm{~cm}$ long and 3-7 cm wide (Barna, 2004).

In total, 36 European beech plots (Fig. 2.2 and Table 2.1) spanning from young to mature stands were selected. The canopy height model of all plots (Fig 2.3) were generated following the pit-free algorithm using the 2017 summer airborne LiDAR data (Khosravipour et al., 2014). It can be seen that these plots are of varying tree density and tree height (Fig 2.3). 


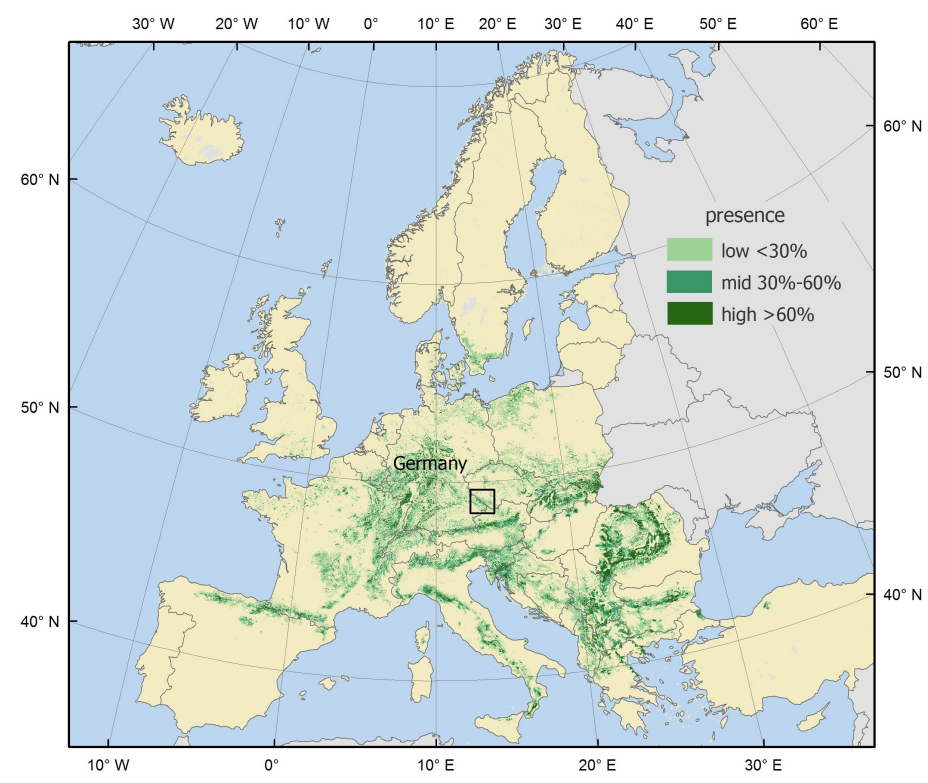

Figure 2.1: The distribution of European beech (Fagus sylvatica) and the location of the study area Bavarian Forest National Park in Europe

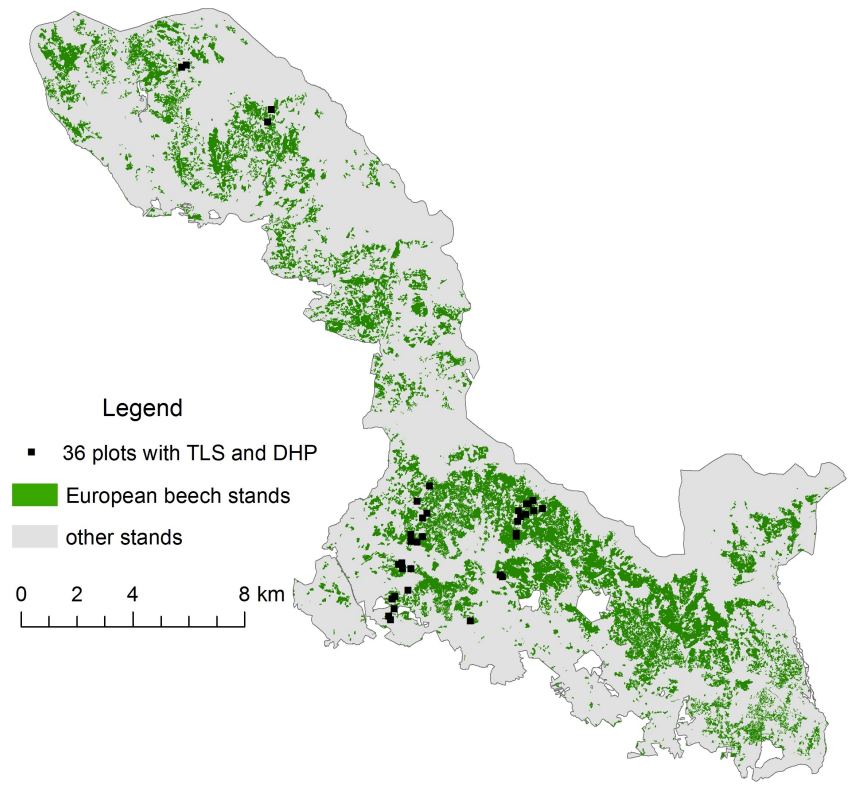

Figure 2.2: The distribution of the 36 European beech plots with both TLS and DHP data 


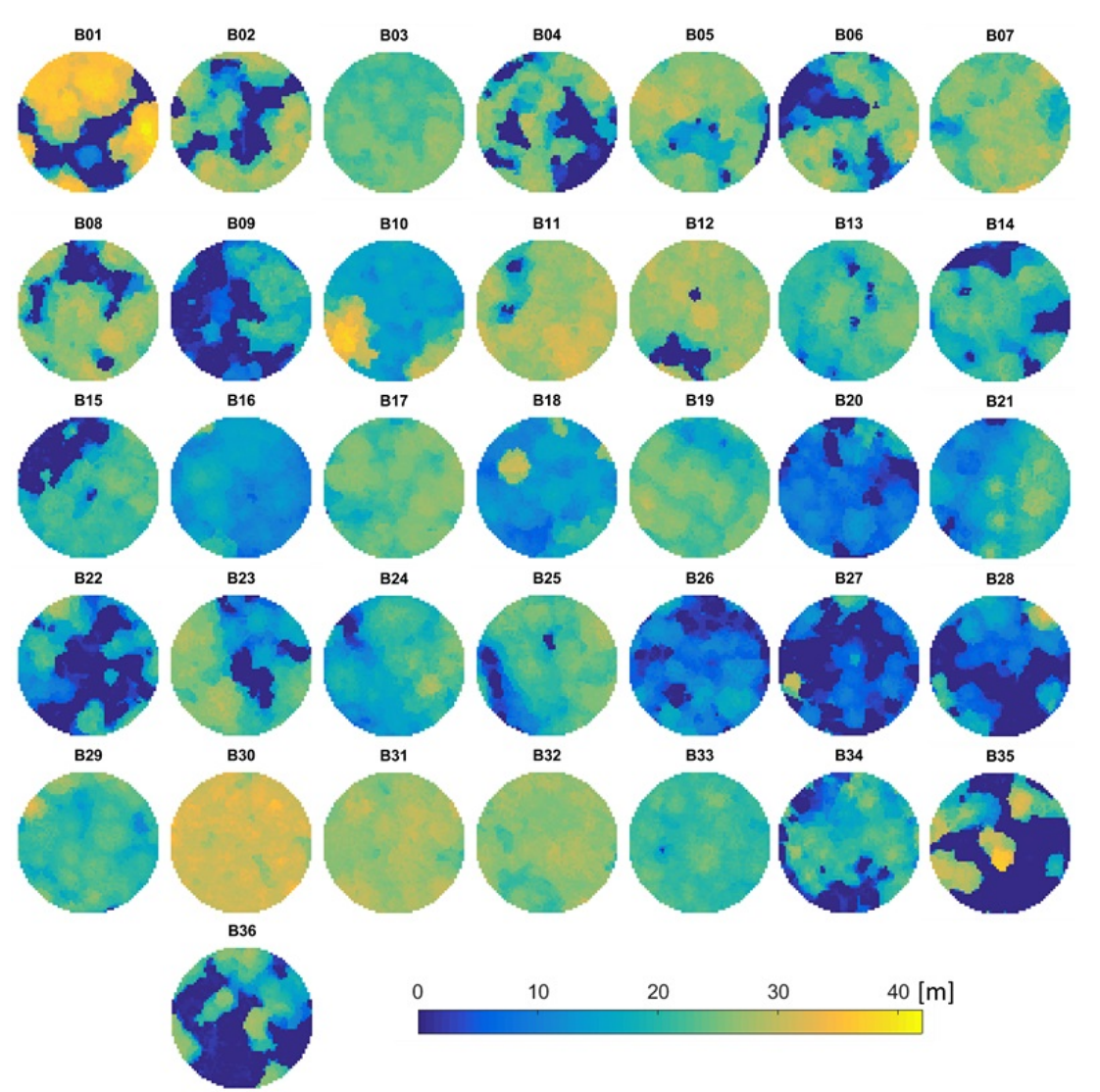

Figure 2.3: Canopy height models of the 36 European beech plots of different structures 
Table 2.1: Basic information of the 36 European beech plots

\begin{tabular}{|c|c|c|c|c|c|}
\hline Plot Name & MedianH [m] & StdH [m] & Elevation [m] & Median Slope $\left[{ }^{\circ}\right]$ & $\mathrm{N} / \mathrm{ha}$ \\
\hline B01 & 33.6 & 11.5 & 808.3 & 9.2 & 280 \\
\hline В02 & 25.6 & 7 & 822.6 & 4.4 & 380 \\
\hline В03 & 23.4 & 1.7 & 844.6 & 8.9 & 540 \\
\hline B04 & 22.6 & 9.9 & 862.6 & 8.7 & 460 \\
\hline B05 & 25.6 & 4.8 & 836.2 & 6.1 & 420 \\
\hline B06 & 22.2 & 9.9 & 839.4 & 4.9 & 380 \\
\hline В07 & 25.7 & 3.7 & 853.5 & 9.8 & 280 \\
\hline B08 & 25.7 & 7.2 & 847.7 & 14.2 & 280 \\
\hline B09 & 8 & 8.6 & 829.7 & 7.7 & 540 \\
\hline B10 & 16 & 6.8 & 869.2 & 8.8 & 1840 \\
\hline B11 & 27.5 & 4.5 & 841.5 & 5.7 & 640 \\
\hline B12 & 26.8 & 5.8 & 851 & 9 & 220 \\
\hline B13 & 21.6 & 4 & 915.9 & 14.5 & 820 \\
\hline B14 & 20.2 & 7.3 & 964.2 & 11 & 320 \\
\hline B15 & 20.8 & 7.7 & 1133.9 & 17.6 & 1140 \\
\hline B16 & 13 & 3.2 & 990.9 & 11.8 & 2200 \\
\hline B17 & 24.4 & 2.5 & 1019.7 & 11 & 460 \\
\hline B18 & 13.6 & 5.1 & 781.9 & 5.3 & 980 \\
\hline B19 & 23.3 & 3.2 & 1030.4 & 16.1 & 480 \\
\hline B20 & 8.2 & 4.6 & 792.9 & 5.7 & 520 \\
\hline B21 & 17.1 & 5.5 & 826.8 & 7.5 & 420 \\
\hline B22 & 11 & 8.7 & 892.4 & 9.7 & 260 \\
\hline B23 & 21.3 & 8 & 962.3 & 14.2 & 240 \\
\hline B24 & 18.6 & 5.1 & 1043.9 & 18.4 & 1140 \\
\hline B25 & 22 & 5.7 & 972.4 & 8.5 & 540 \\
\hline B26 & 8.3 & 5 & 912.4 & 11.5 & 500 \\
\hline $\mathrm{B} 27$ & 4.2 & 6.1 & 866.6 & 8.3 & 240 \\
\hline B28 & 6.9 & 7.4 & 796.4 & 6.4 & 400 \\
\hline B29 & 21.3 & 3 & 911.7 & 7.7 & 480 \\
\hline B30 & 31.1 & 1.5 & 775.7 & 13.6 & 480 \\
\hline B31 & 27.3 & 1.7 & 981.8 & 9.3 & 520 \\
\hline B32 & 26.2 & 1.9 & 834.1 & 4.1 & 420 \\
\hline B33 & 21.4 & 1.6 & 1082 & 9.2 & 960 \\
\hline B34 & 18.4 & 8.2 & 1096.3 & 15.5 & 660 \\
\hline B35 & 18.5 & 12.7 & 982.1 & 18 & 360 \\
\hline B36 & 11.6 & 10.6 & 1050.9 & 18 & 320 \\
\hline
\end{tabular}

MedianH: plot median canopy height; StdH: plot standard deviation of canopy height

N/ha: number of trees per hectare

\subsubsection{TLS and DHP data collection}

Fieldwork was conducted from 17-July-2017 to 9-August-2017, in leaf-on conditions. Both TLS and DHP data were collected in all the 36 beech stands. The TLS used is Riegl VZ-400, with the laser pulse at $1550 \mathrm{~nm}$ wavelength and a beam divergence of $0.3 \mathrm{mrad}$. The range accuracy is $5 \mathrm{~mm}$. The angular step in both vertical and horizontal directions was 
set as $0.04^{\circ}$. At each plot, there was one center and three triangular scan positions. To achieve co-registration of these four scans, 12-18 retro-reflective targets were placed in the plot to serve as control points. All TLS scans were taken during calm conditions to prevent wind effects on leaves (Wilkes et al., 2017). On average, it took 1-1.5 hours to finish four scans in one plot. After TLS data collection and preprocessing, a point cloud with a radius of $15 \mathrm{~m}$ was clipped in each plot.

In each plot, 12-20 upward-pointing hemispherical photos were collected, first in the plot center, and then $10 \mathrm{~m}$ from the center in each diagonal direction. In order to reduce gap fraction uncertainty caused by various reasons (Woodgate et al., 2015) such as sky illumination conditions and image classification method, DHP was carefully setup as follows. At each position, a Canon EOS 5D camera equipped with a Sigma $8 \mathrm{~mm}$ F3.5 EX fish-eye lens was leveled on a tripod between 1 and $1.3 \mathrm{~m}$ above ground. All photos were taken in diffuse light conditions at dusk, or overcast diffuse conditions during cloudy days, using manual exposure. Each image had a high resolution of 5600 by 3898 pixels.

\subsection{Synthetic dataset}

In order to acquire the $100 \%$ true leaf inclination reference for evaluating the TLS and DHP method accuracies, synthetic datasets are generated through two steps. First, a series of virtual scenes of different canopy structures are created. Second, DHP and TLS point clouds are simulated using these virtual scenes.

\subsubsection{Virtual scene construction}

In this study, 24 virtual scenes are created following the method described by Widlowski et al. (2007) and Leblanc and Fournier (2014). These 24 scenes include 8 homogeneous stands and 16 heterogeneous stands. The 8 homogeneous stands are characterized by closed canopies, an even canopy height, an even local vegetation density, and approach a turbid media. The 16 heterogeneous stands, on the contrary, consist of a discontinuous canopy, uneven tree spacing and uneven local vegetation density. 
In all virtual scenes, the basic leaf unit remains the same. The leaf has a diamond shape with an area of $20 \mathrm{~cm}^{2}$. The diamond shape consists of only two triangles. This is used due to a compromise between similarity to real leaf shape and computation time. This choice is similar to other simulation studies (Gastellu-Etchegorry et al., 2015). In the 8 homogenous stands, the canopy can be regarded as one continuous layer filled with leaves (Fig 2.4 e) and g)). Leaves are randomly distributed with a uniform azimuth orientation. The zenith orientations (i.e. inclination) of leaves follow a predefined LAD function, with the average inclination angle $(\bar{\theta})$ ranging from about $15^{\circ}$ to $70^{\circ}$ (Fig 2.4 a) and c)). In the 16 heterogeneous stands, the stand consists of many randomly distributed and non-overlapping trees (Fig $2.4 \mathrm{f}$ ) and h)). All trees are of the same height, but tree spacing is random. For simplicity, each tree is modeled as a cone-shape trunk with an ellipsoidal crown. Each crown is a turbid medium, filled with randomly distributed leaves. Similarly, the leaves have a uniform azimuth orientation, while the inclination follows a predefined LAD, with $\bar{\theta}$ ranging from about $15^{\circ}$ to $70^{\circ}$ (Fig $2.4 \mathrm{~b}$ ) and d)) The topography is set as flat in all virtual scenes. More details of each virtual stand can be found in Table 2.3.

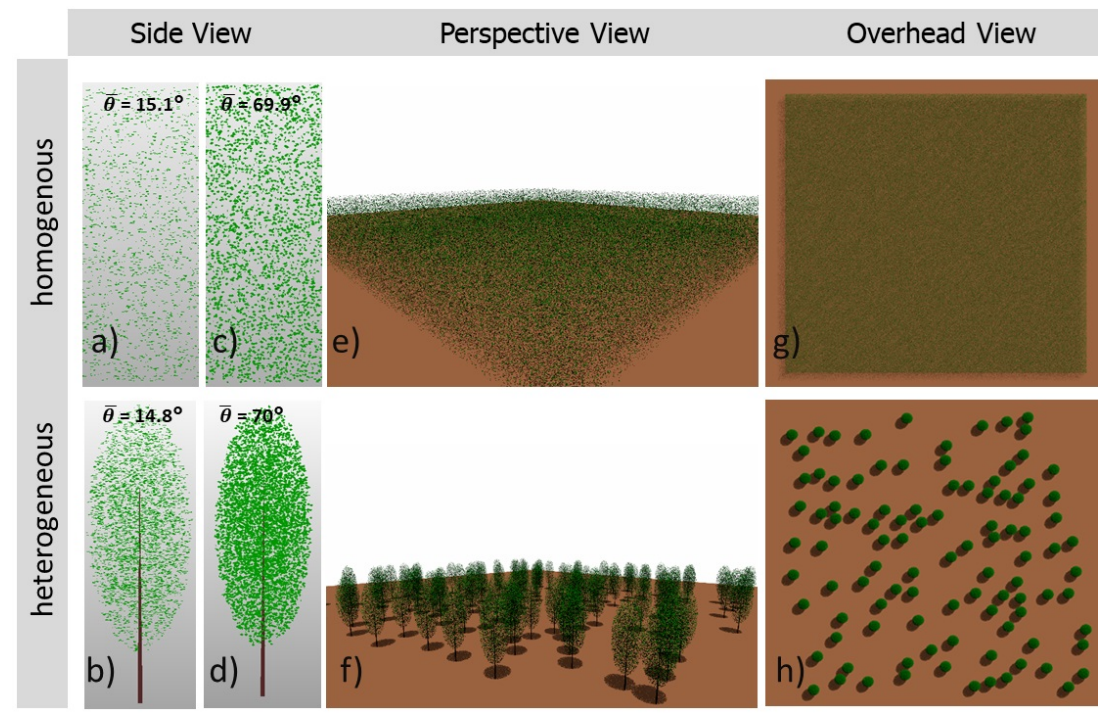

Figure 2.4: Examples of a) c) e) g) virtual homogeneous stands with closed canopy and even canopy height, without any woody material; and b) d) f) h) virtual heterogeneous stands with discontinuous canopy and uneven tree spacing. 


\subsubsection{TLS and DHP simulation}

The synthetic DHP in all virtual stands are simulated by the freeware POV-Ray (www.povray.org). POV-Ray is a ray-tracing program with the fish-eye camera rendering. For each DHP, the camera is placed at a height of $1.5 \mathrm{~m}$ above the ground facing upwards. The horizontal locations of DHPs in a stand follow a grid-pattern (10 m spacing), producing 16 DHPs in a plot. The resolution of all DHPs is 5600 by 3898 pixels, equivalent to the real DHP resolution as collected in the deciduous forests. Two example DHPs in the homogeneous stands and heterogeneous stands can be seen in Fig 2.5 a) and Fig 2.5 b).

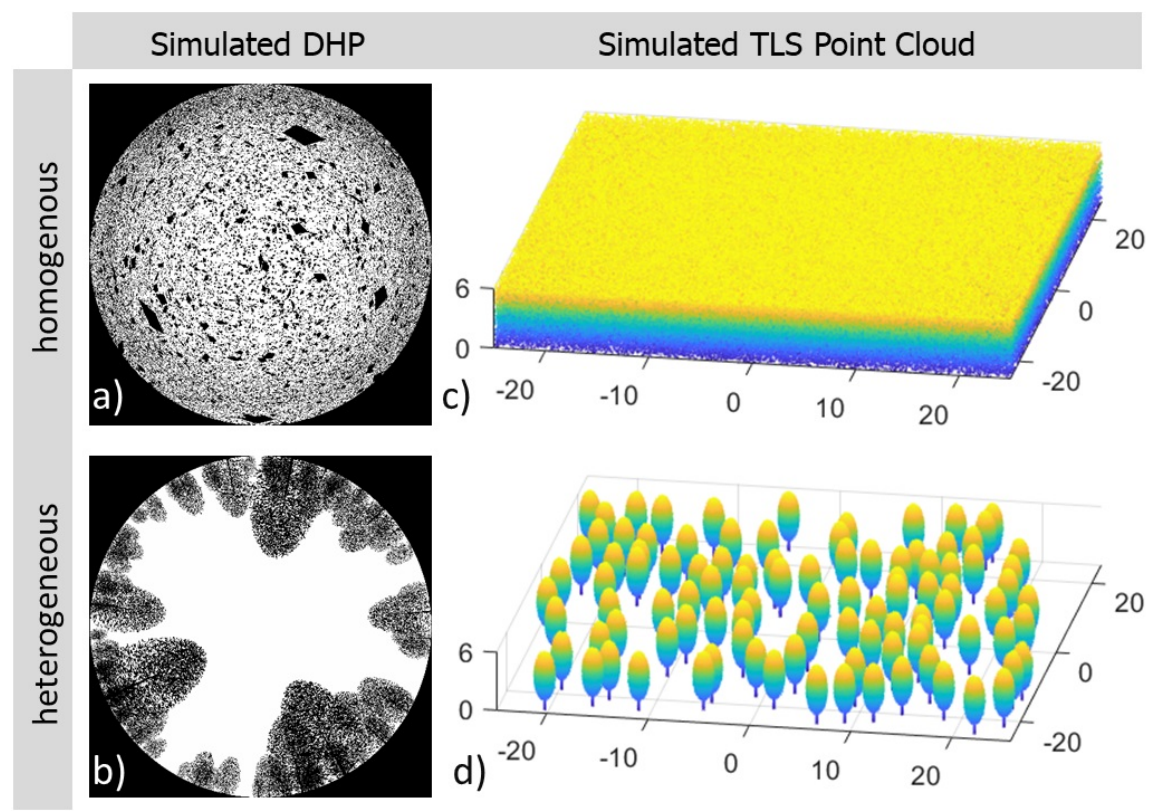

Figure 2.5: Examples of the simulated DHP and simulated TLS point clouds in a) c) virtual homogeneous stands with closed canopy and even canopy height; b) d) virtual heterogeneous stands with discontinuous canopy and uneven tree spacing.

The synthetic TLS point clouds are simulated using the open-source software HELIOS (Bechtold and Höfle, 2016). HELIOS is an easy-to-use LiDAR simulator, which offers various sensor platforms and LiDAR scanners. We use HELIOS to scan all the 24 virtual scenes, using the same settings as used in the beech forest fieldwork (Riegl VZ-400 scanner, 0.3 mrad beam divergence, $0.04^{\circ}$ angular step, $7.5 \mathrm{~m}$ distance away, 4 scan 
positions). Two example TLS point clouds can be seen in Fig 2.5 c) and Fig 2.5 d).

\section{Method}

In this study, field-based forest datasets are first implemented to evaluate the consistency of LAD derived from TLS and DHP. Afterwards, synthetic datasets are designed to gain true reference for evaluating the accuracy of LAD from TLS and DHP. In both datasets, the average inclination angle $(\bar{\theta})$ is calculated from DHP using the gap fraction inversion method, and from TLS using the geometrical method.

The flowchart of this study is displayed in Fig 2.6. In the following sections, details of the LAD calculation methods are introduced.
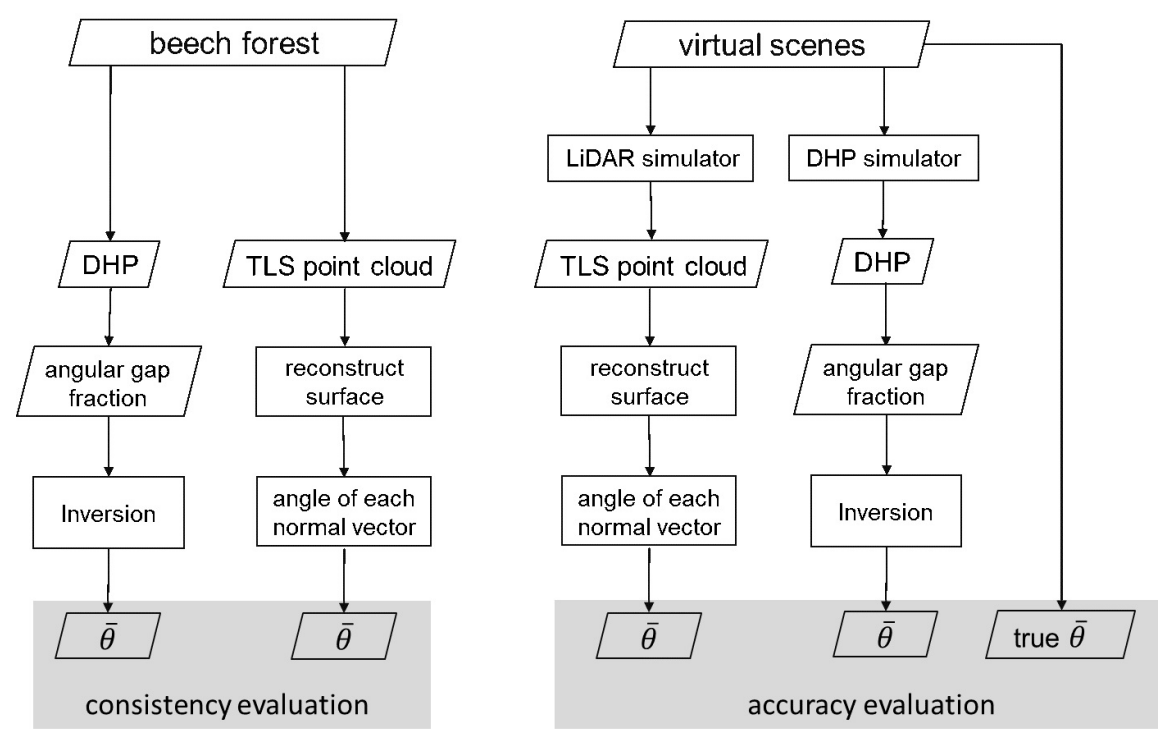

Figure 2.6: The flowchart to evaluate the consistency and accuracy of TLS and DHP in estimating leaf angle distribution (note: $\bar{\theta}$ is the average inclination angle)

\subsection{DHP gap fraction inversion method}

Radiation attenuation is controlled by the canopy structure, forming a theoretical basis to estimate canopy structure from radiation attenuation 
measurements. Using multiple direction measurement, one can retrieve LAD using inversion (Norman and Campbell, 1989; Weiss et al., 2004). Using DHP, the radiation attenuation is approximated as the proportion of gap pixels. We adopt the automatic Two-Corner classification method (Macfarlane, 2011) to classify gap pixels and canopy pixels. This method minimizes subjective thresholding and has been recommended in previous studies (Woodgate et al., 2015). The classified images are afterwards broken into 18 annuli, each of $5^{\circ}$. The gap fraction in $\theta$ direction is calculated using,

$$
P_{\text {gap }}(\theta)=\frac{N_{\text {gap }}(\theta)}{N_{\text {gap }}(\theta)+N_{\text {canopy }}(\theta)}
$$

where $N_{\text {gap }}(\theta)$ is the number of gap pixels in the $\theta$ annulus, $N_{\text {canopy }}(\theta)$ is the number of canopy pixels in the $\theta$ annulus. For each plot, after averaging the angular $P_{\text {gap }}$ from $0^{\circ}$ to $90^{\circ}$ in all DHPs, the plot averaged angular $P_{\text {gap }}$ profile is calculated. Due to the high portion of mixed pixels above $80^{\circ}$, the annuli from $80^{\circ}$ to $90^{\circ}$ are removed from subsequent analysis. Using the $P_{\text {gap }}(\theta)$ from $0^{\circ}$ to $80^{\circ}$, LAD inversion is conducted based on the following equations (Weiss et al., 2004; Leblanc and Fournier, 2017),

$$
\begin{aligned}
& P_{\text {gap }}(\theta)=e^{-G(\theta) L_{\mathrm{eff}} / \cos (\theta)} \\
& L_{\mathrm{eff}}=\lambda L \\
& G(\theta)=\frac{\left(\chi^{2}+\tan ^{2} \theta\right)^{0.5} \cos \theta}{\chi+1.774(\chi+1.182)^{-0.73}} \\
& c_{\text {gap }}=\left(P_{\text {gap_meas }}-P_{\text {gap_mod }}\right) \cdot\left(P_{\text {gap_meas }}-P_{\text {gap_mod }}\right)^{T}
\end{aligned}
$$

where $P_{\text {gap }}(\theta)$ is the gap fraction in zenith direction $\theta, L_{\text {eff }}$ is the effective LAI (or effective PAI if there are woody elements), $L$ is the true LAI and $\lambda$ is the clumping index. The $\lambda$ value depends on the canopy structure. It is smaller than 1 for aggregated canopies, while greater than 1 for regular dispersed canopies. $G(\theta)$ is the leaf projection function in direction $\theta$, 
$P_{\text {gap_meas }}$ is the measured gap fraction and $P_{\text {gap_mod }}$ is the modeled gap fraction at different zenith directions (Fournier and Hall, 2017). Using the ellipsoidal function, $G(\theta)$ is determined by only one parameter $\chi$, which is determined by the average inclination angle $\bar{\theta}$ (Campbell, 1986; Wang et al., 2007). Using numerical optimization, $\chi$ and $L_{\text {eff }}$ are inversed from nonlinear least-squares method by minimizing the cost function $c_{\text {gap }}$, from $P_{\text {gap }}$ observed at different $\theta$ angles. Then, $\bar{\theta}$ is calculated via,

$$
\bar{\theta}=90\left(0.1+0.9 e^{-0.5 x}\right)
$$

For more details, one can refer to previous literature (Weiss et al., 2004; Campbell et al., 1989; Wang et al., 2007; Leblanc and Fournier, 2017).

\subsection{TLS geometrical method}

After TLS data collection, a series of preprocessing steps are conducted. First, the point clouds from multiple scan positions are merged together by the Riscan software (http://www.riegl.com). To preserve the topology, topographic normalization is not conducted (Liu et al., 2017a). After noisy point removal, the point cloud is used for subsequent analysis. Individual leaf surface and woody surface is reconstructed through fitting a plane in a set of neighboring points. The normal vector of each leaf or woody surface is calculated through principal component analysis on the Cartesian coordinates of all the points in this surface (Klasing et al., 2009). The direction of the normal vector is the same as the direction of the eigenvector with the minimum eigenvalue. After retrieving the inclination angles of all the surfaces, the average inclination angle $\bar{\theta}$ is calculated using,

$$
\begin{aligned}
& \bar{\theta}=\frac{\sum_{i=1}^{N_{\text {total }}} \omega_{i} \theta_{i}}{\sum_{i=1}^{N_{\text {total }}} i} \\
& \omega_{i}=\frac{1}{N_{\text {neighbor } \_i}}
\end{aligned}
$$

where $\theta_{i}$ is the inclination angle of the reconstructed surface for point $i, N_{\text {total }}$ is the total number of points in the TLS point cloud, $\omega_{i}$ is a weight value correcting the local point density variation, $N_{\text {neighbor_i }}$ is the number of neighboring points for point $i$ within a neighborhood size of 
$0.04 \mathrm{~m}$. The reason for weighting and correcting point density variation is referred to a previous study (Bailey and Mahaffee, 2017).

\subsection{Consistency and accuracy assessment}

First, a consistency evaluation using the field based dataset is conducted by comparing the average inclination angle $(\bar{\theta})$ estimated from TLS and from DHP. Furthermore, an accuracy evaluation using the synthetic dataset is conducted. The estimates of $\bar{\theta}$ from TLS and from DHP are tested against the true pre-defined reference values of $\bar{\theta}$ in the virtual stands. The linear regression line is forced to the origin $(y=x)$. Then, the coefficient of determination $\left(R^{2}\right)$ and the root mean square error (RMSE) are calculated to compare whether the TLS or DHP method has higher accuracy.

\section{Results}

\subsection{The consistency of LAD estimated from TLS and DHP using the field based dataset}

Using the field based forest dataset, the results of LAD estimated from DHP and TLS are displayed in Table 2.2 and Fig 2.7. There is a severe inconsistency in the retrieved average inclination angle $\bar{\theta}(r=0.19, p=0.26)$. Further inspection reveals that, the $\bar{\theta}$ values are in quite different data ranges $\left(\bar{\theta} \in\left(44^{\circ}, 53^{\circ}\right)\right.$ from TLS, and $\bar{\theta} \in\left(18^{\circ}, 76^{\circ}\right)$ from DHP). 
Table 2.2: Summary of the LAD results estimated from TLS and DHP in the field based dataset of 36 European beech stands

\begin{tabular}{llllll}
\hline Plot Name & $\bar{\theta}_{\text {TLS }}\left[^{\circ}\right]$ & $\bar{\theta}_{\text {DHP }}\left[^{\circ}\right]$ & Plot Name & $\bar{\theta}_{\text {TLS }}\left[^{\circ}\right]$ & $\bar{\theta}_{\text {DHP }}\left[^{\circ}\right]$ \\
\hline B01 & 44.13 & 56.11 & B19 & 47.13 & 38.00 \\
B02 & 47.86 & 53.12 & B20 & 49.49 & 37.62 \\
B03 & 50.68 & 50.74 & B21 & 46.75 & 53.04 \\
B04 & 48.16 & 32.01 & B22 & 45.94 & 56.25 \\
B05 & 47.80 & 39.84 & B23 & 46.84 & 34.03 \\
B06 & 46.99 & 18.34 & B24 & 48.82 & 37.24 \\
B07 & 47.26 & 75.86 & B25 & 48.82 & 35.66 \\
B08 & 44.77 & 21.41 & B26 & 47.21 & 56.09 \\
B09 & 47.76 & 25.05 & B27 & 51.09 & 46.86 \\
B10 & 49.99 & 55.67 & B28 & 49.23 & 55.27 \\
B11 & 47.51 & 54.62 & B29 & 49.41 & 59.58 \\
B12 & 47.00 & 60.23 & B30 & 50.32 & 59.31 \\
B13 & 52.65 & 52.69 & B31 & 46.55 & 23.80 \\
B14 & 47.71 & 59.86 & B32 & 47.74 & 25.05 \\
B15 & 49.89 & 34.49 & B33 & 48.02 & 53.12 \\
B16 & 52.12 & 52.78 & B34 & 47.92 & 61.75 \\
B17 & 48.84 & 32.86 & B35 & 47.58 & 64.60 \\
B18 & 49.44 & 34.60 & B36 & 50.21 & 59.40 \\
\hline
\end{tabular}

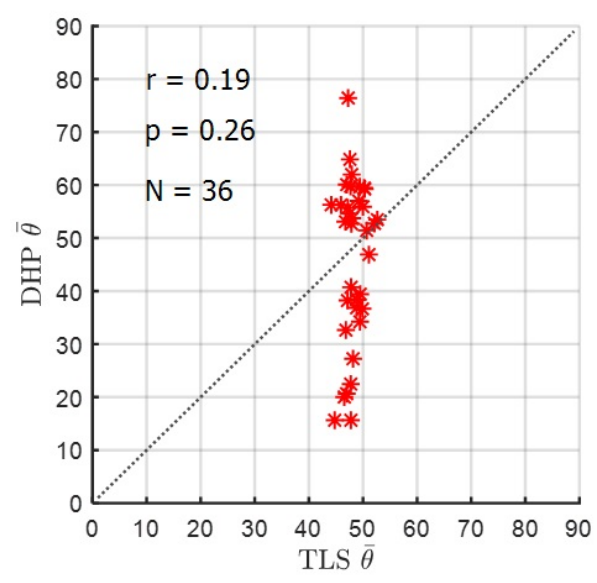

Figure 2.7: The average inclination angle $(\bar{\theta})$ estimated from TLS and from DHP using the field based dataset in the 36 European beech stands 


\subsection{The accuracy of LAD estimated from TLS and DHP using the synthetic dataset}

The estimated LAD results from the synthetic dataset are detailed in Fig 2.8 and Table 2.3. The average inclination angle $(\bar{\theta})$ is used to represent the LAD results. Pre-defined true $\bar{\theta}$ values and the estimated $\bar{\theta}$ values from simulated TLS and simulated DHP are displayed in the scatter plots. The TLS method has much higher $R^{2}$ and lower RMSE than the DHP method $\left(R^{2}: 0.79>0.12\right.$; RMSE: $\left.6.41^{\circ}<16.40^{\circ}\right)$ when estimating $\bar{\theta}$. Upon further observation, TLS tends to overestimate $\bar{\theta}$ in canopies with more flat growing leaves, while underestimating $\bar{\theta}$ in canopies with more vertical leaves. DHP works very well in homogeneous stands that approach a turbid media, with the results locating close to the 1:1 line. However, in heterogeneous forest stands, the performance of DHP deteriorates, decreasing the accuracy. Overall, the synthetic datasets demonstrate that TLS more accurately estimates LAD in comparison to DHP.
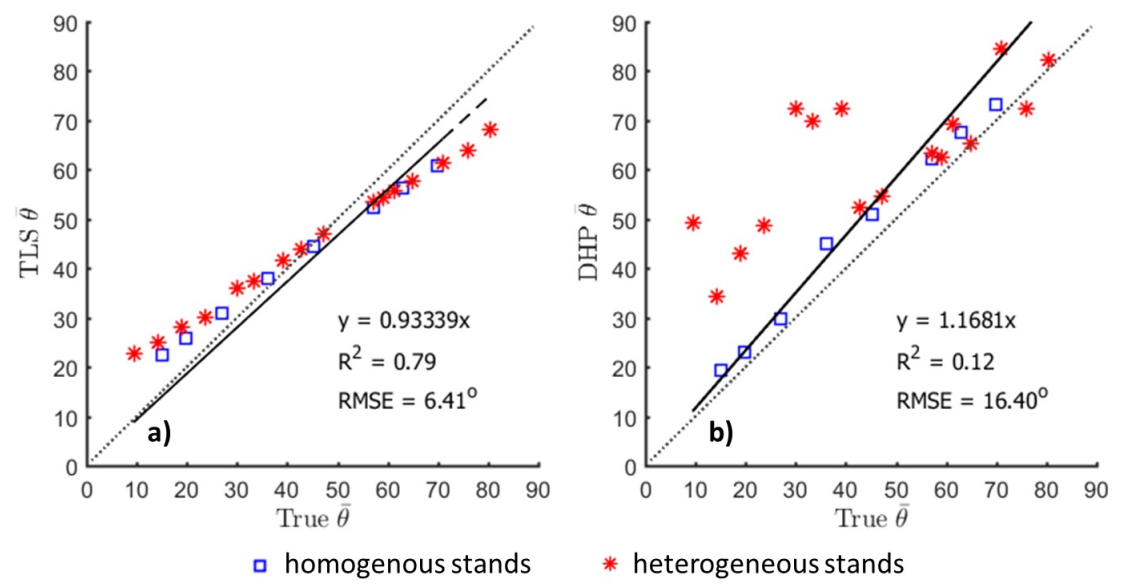

Figure 2.8: The LAD results accuracy from TLS and DHP in the synthetic dataset. a) the estimated average inclination angle $(\bar{\theta})$ from TLS; b) the estimated $\bar{\theta}$ from DHP 
Table 2.3: Summary of the LAD results estimated from TLS and DHP in the synthetic dataset of 24 virtual stands

\begin{tabular}{|c|c|c|c|c|c|c|c|c|c|}
\hline Plot Type & Plot Name & $\begin{array}{l}\text { Stand } \\
{[\mathrm{m}]}\end{array}$ & Size & $R_{\text {plot }}[\mathrm{m}]$ & $H[\mathrm{~m}]$ & LAI & $\bar{\theta}_{\text {true }}\left[^{\circ}\right]$ & $\bar{\theta}_{\mathrm{TLS}}\left[{ }^{\circ}\right]$ & $\bar{\theta}_{\mathrm{DHP}}\left[{ }^{\circ}\right]$ \\
\hline \multirow{9}{*}{$\begin{array}{l}\text { Homogeneous } \\
\text { stands }\end{array}$} & $\mathrm{T} 1$ & $50 \times 50$ & & 15 & 6 & 0.5 & 15.1 & 22.44 & 15.65 \\
\hline & $\mathrm{T} 1$ & $50 \times 50$ & & 15 & 6 & 0.5 & 15.1 & 22.44 & 15.65 \\
\hline & $\mathrm{T} 2$ & $50 \times 50$ & & 15 & 6 & 0.5 & 19.7 & 25.93 & 16.25 \\
\hline & T3 & $50 \times 50$ & & 15 & 6 & 0.5 & 27 & 30.85 & 25.85 \\
\hline & $\mathrm{T} 4$ & $50 \times 50$ & & 15 & 6 & 0.5 & 36.1 & 38 & 37.7 \\
\hline & T5 & $50 \times 50$ & & 15 & 6 & 0.5 & 45.3 & 44.36 & 49.02 \\
\hline & $\mathrm{T} 6$ & $50 \times 50$ & & 15 & 6 & 0.5 & 57.2 & 52.3 & 59.93 \\
\hline & $\mathrm{T} 7$ & $50 \times 50$ & & 15 & 6 & 0.5 & 62.9 & 56.35 & 64.87 \\
\hline & $\mathrm{T} 8$ & $50 \times 50$ & & 15 & 6 & 0.5 & 69.9 & 60.74 & 71.97 \\
\hline \multirow{16}{*}{$\begin{array}{l}\text { Heterogeneous } \\
\text { stands }\end{array}$} & F1 & $50 \times 50$ & & 15 & 6 & 0.5 & 9.42 & 22.6 & 49.15 \\
\hline & F2 & $50 \times 50$ & & 15 & 6 & 0.5 & 14.27 & 24.87 & 34.37 \\
\hline & F3 & $50 \times 50$ & & 15 & 6 & 0.5 & 18.98 & 28.03 & 43.15 \\
\hline & $\mathrm{F} 4$ & $50 \times 50$ & & 15 & 6 & 0.5 & 23.72 & 30.04 & 48.56 \\
\hline & F5 & $50 \times 50$ & & 15 & 6 & 0.5 & 29.96 & 36.06 & 72.35 \\
\hline & F6 & $50 \times 50$ & & 15 & 6 & 0.5 & 33.25 & 37.46 & 69.82 \\
\hline & F7 & $50 \times 50$ & & 15 & 6 & 0.5 & 39.16 & 41.68 & 72.48 \\
\hline & F8 & $50 \times 50$ & & 15 & 6 & 0.5 & 42.83 & 43.83 & 52.3 \\
\hline & F9 & $50 \times 50$ & & 15 & 6 & 0.5 & 47.3 & 46.86 & 54.72 \\
\hline & F10 & $50 \times 50$ & & 15 & 6 & 0.5 & 57.31 & 53.34 & 63.35 \\
\hline & F11 & $50 \times 50$ & & 15 & 6 & 0.5 & 59.24 & 54.17 & 62.48 \\
\hline & F12 & $50 \times 50$ & & 15 & 6 & 0.5 & 61.31 & 55.72 & 69.21 \\
\hline & F13 & $50 \times 50$ & & 15 & 6 & 0.5 & 64.98 & 57.76 & 65.22 \\
\hline & F14 & $50 \times 50$ & & 15 & 6 & 0.5 & 70.99 & 61.48 & 84.56 \\
\hline & F15 & $50 \times 50$ & & 15 & 6 & 0.5 & 75.93 & 63.89 & 72.26 \\
\hline & F16 & $50 \times 50$ & & 15 & 6 & 0.5 & 80.5 & 68.24 & 82.17 \\
\hline
\end{tabular}

$R_{\text {plot }}:$ radius of the plot; $H$ : canopy height

\section{Discussion}

\subsection{Cause of inconsistency of LAD estimated from TLS and DHP}

From the field based dataset, DHP and TLS produce quite inconsistent LAD estimates $(r=0.19, p=0.26)$ in the European beech forests. Since in natural forests, it is impossible to acquire $100 \%$ true reference value on ground, we cannot conclude which method is more accurate. The estimated $\bar{\theta}$ from DHP ranges in $\left(18^{\circ}, 76^{\circ}\right)$, the extremely high $\bar{\theta}$ values implies most beech leaves and woody branches grow vertically in the stand, which is very unlikely in real beech forest canopies. It contradicts previous studies, where the average leaf inclination angle is reported to be around $21.7^{\circ}$ in European beech forests in center Germany (Wagner and Hagemeier, 2006). It also contradicts a previous study which reports the average branch inclination angles of European beech ranges in $\left(50.7^{\circ}\right.$, 
$59.8^{\circ}$ ) in east Germany (Levia et al., 2015). As such, it may be reasonable for us to hypothesize that TLS is more accurate than DHP in estimating LAD.

From the synthetic dataset, we demonstrate that TLS estimates LAD more accurately than DHP in broadleaf forests. DHP only produces accurate $\bar{\theta}$ estimates in homogeneous stands (similar to the turbid media). In these stands, the canopy is of even height, even local plant density, and has no large between-crown gaps, which is rather unusual in real forest stands. However, in heterogeneous forest stands with uneven tree spacing, uneven local plant density and large between-crown gaps, the DHP method estimates $\bar{\theta}$ very poorly. In contrast, TLS accurately estimates $\bar{\theta}$ in both types of forest stands. There is a trend of overestimation of $\bar{\theta}$ for low values. This is perhaps due to more difficulty in generating returns for flat leaves than for erect leaves. The plot F16 $\left(\bar{\theta}=80.5^{\circ}\right)$ has 1.8 times points than the plot F2 $\left(\bar{\theta}=14.27^{\circ}\right)$. This results in higher contribution of large leaf angles. The reason for underestimation of $\bar{\theta}$ for high values currently remains unknown to us, which requires further research.

The abnormal $\bar{\theta}$ results from DHP in heterogeneous forest stands are very likely caused by a violation of the "path length" calculation, which assumes an even tree height, even tree spacing and flat topography for the canopy. In the DHP method, a key factor determining the radiation attenuation (or gap fraction) through canopies is the path length the light has to travel. Let $L_{1}$ and $L_{2}$ be the path length at zenith angle $\theta_{1}$ and $\theta_{2}$. DHP cannot provide the exact values of $L_{1}$ and $L_{2}$ due to lens projection. Instead, DHP approximates the ratio of $L_{1}$ and $L_{2}$ as the ratio of $\cos \left(\theta_{1}\right)$ and $\cos \left(\theta_{2}\right)$, in Fig 2.9 a). This forms the basis for Eq 2.2 in Section 3.1 in this chapter. However, this approximation is only valid in homogeneous stands with even canopy height, even tree spacing, and a flat topography (Chen et al., 1991). But for forests with complex structure, it is very common that the real stands are more similar to the case in Fig 2.9 b). In such stands, the uneven canopy height, uneven between-crown gaps, and rugged topography make the DHP method assumption invalid. To sum up, the complex canopy and topography structure in natural forests undermines the basis to use the DHP method.

Our results reveal that for the synthetic dataset, the correlation between LAD estimated from DHP and TLS was much higher than in the real 


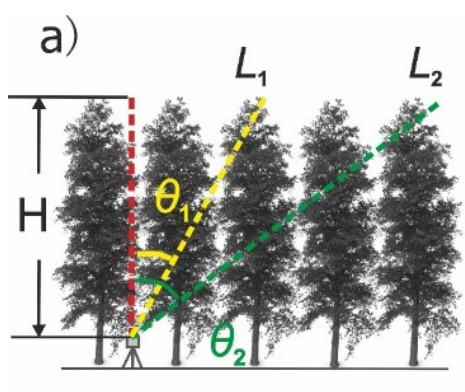

$L_{1} / L_{2}=\cos \left(\theta_{2}\right) / \cos \left(\theta_{1}\right)$

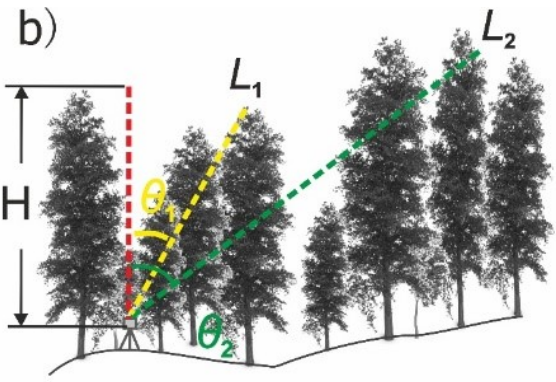

$L_{1} / L_{2} \neq \cos \left(\theta_{2}\right) / \cos \left(\theta_{1}\right)$

Figure 2.9: The violation of path length assumption in DHP due to uneven tree height, uneven tree spacing and non-flat underneath topography in heterogeneous forests

forest dataset (Fig 2.10 and Fig 2.7). There may be several reasons for the greater inconsistency in the field based dataset. First, unlike the flat topography in all the virtual scenes, in the real forests there is higher slope variation $\left(4.44^{\circ}-19.35^{\circ}\right)$. This makes the path length issue worse. Second, the DHP classification accuracy may be lower in real forests than in virtual forests. Although special care is taken in the field, the illumination levels, exposures and the reflectance of leaves may differ among different stands. These all affect the classification accuracy and further affect the gap fraction value. However, in the synthetic dataset, the above-mentioned factors are kept the same to avoid uncertainty in DHP processing. Third, the real LAD in the European beech forests may not have a variation as large as $\left[5^{\circ}, 80^{\circ}\right]$ in the virtual forests. This would also decrease the correlation. The positive bias in the $\bar{\theta}$ estimated from DHP than from TLS (Fig 2.10) is perhaps caused by the angular dependence of $L_{e f f}$. In the inversion, $L_{e f f}$ was assumed constant in all directions. This may not be valid in heterogeneous stands since the clumping index $\lambda$ may decrease with $\theta$ (Frazer et al., 2017).

\subsection{Comparison of TLS and DHP in estimating LAD}

Although this study has demonstrated TLS estimates LAD more accurately than DHP, this conclusion applies only for broadleaf species. TLS uses a geometrical method to detect canopy structure. The underlying requirement is that there are sufficient data sampling of plant organs. 


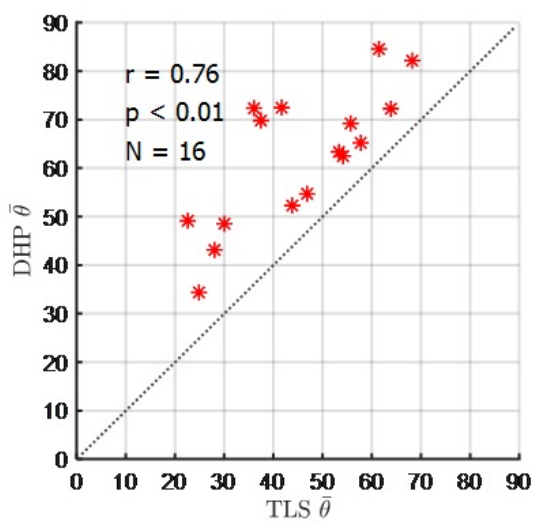

Figure 2.10: Inconsistency of the average inclination angle $(\bar{\theta})$ estimated from TLS and from DHP using the synthetic forest datasets

For coniferous forests, it is currently difficult to generate small and dense returns on each needle. But the structure of individual branches can be retrieved (Zhu et al., 2018b). In this study, the leaf size ranged in 20-40 $\mathrm{cm}^{2}$, so future studies could explore the impact of leaf size and shape on the accuracy of leaf angle estimation. In addition, to ensure a high point density in dense canopies, utilizing multiple scan positions is strongly recommended.

DHP is a conventional technique, which has been widely used in estimating canopy structure. However, a key limitation of DHP is its assumption that a 2D medium can be used to infer a 3D environment (Fournier and Hall, 2017). The basic principle of DHP is based on gap fraction inversion. As a result, the following two aspects both greatly influence the estimations. The first factor is an accurate gap fraction measurement. The second factor is an appropriate radiative transfer theory to inverse the gap fraction (Norman and Campbell, 1989). In the past 20 years, many studies have proven the challenge and high uncertainty when measuring gap fraction using DHP, including photo numbers, positions and averaging methods (Weiss et al., 2004), camera exposure (Zhang et al., 2005), and image classification methods (Wagner and Hagemeier, 2006; Woodgate et al., 2015). However, few studies have investigated whether the canopy of interest fits the DHP radiative transfer theory or not. In the early research, Chen et al. (1991) clarified their study area as “... the forest floor was horizontal and the canopy was homogeneous in terms 
of tree height and spacing". Nevertheless, most follow-up studies fail to take this limitation of the DHP method into account (Beaudet and Messier, 2002; Macfarlane et al., 2007; Woodgate et al., 2017). Regarding non-flat topography, although some studies try to correct for the path length variation over slope terrains, a uniform slope is often assumed (Schleppi et al., 2007; Gonsamo and Pellikka, 2008), which is not applicable to rugged topography. In addition, it is currently not possible to correct for uneven tree height and uneven tree spacing effect using DHP.

Due to the high uncertainty in the DHP gap fraction measurement and the limited applicability of the inversion model, we suggest to develop and use TLS methods rather than DHP methods in estimating leaf angle distribution for broad leaf forests. A summary of the comparison between the two techniques is shown in Table 2.4.

Table 2.4: Comparison of TLS and DHP in measuring leaf angle distribution (LAD)

\begin{tabular}{lll}
\hline & TLS & DHP \\
\hline equipment cost & high & medium \\
equipment weight & high & low \\
disturbance to canopy & no & no \\
sampling time & long (usually $>1$ hour) & short (usually $<1$ hour) \\
canopy type & broadleaf & broadleaf or coniferous \\
individual leaf angle & yes & no \\
3D information & yes & no \\
ability to differ woody & yes & no \\
requirement & high point density & similar tree height, tree \\
& & spacing, flat or uniform \\
& & slope \\
\hline
\end{tabular}

\section{Conclusion}

This study evaluates which in-situ technique, either terrestrial LiDAR (TLS) or digital hemispherical photography (DHP), estimates the leaf angle distribution (LAD) more accurately. To achieve this, both field based broadleaf forest dataset and synthetic (virtual) dataset are used to evaluate the consistency and accuracy of LAD. Real TLS and DHP data are collected in 36 European beech stands, covering a wide range of natural forest structure. From the results, there is inconsistency 
of the average inclination angle $(\bar{\theta})$ retrieved from TLS and DHP $(\bar{\theta} \in$ $\left(44^{\circ}, 53^{\circ}\right)$ from TLS, and $\bar{\theta} \in\left(18^{\circ}, 76^{\circ}\right)$ from DHP). Due to lack of a true reference value, it cannot be concluded which technique is more accurate. Further experiments are conducted using 24 virtual scenes, including homogeneous stands and heterogeneous stands. The results demonstrate that TLS produces much more accurate LAD results than DHP $\left(R^{2}: 0.79>0.12\right.$; RMSE: $\left.6.41^{\circ}<16.40^{\circ}\right)$. DHP can produce reliable results in homogeneous stands featured by even canopy height and closed canopy. Nevertheless, DHP cannot produce accurate estimates of LAD in heterogeneous stands with discontinuous canopy and an uneven tree spacing. A plausible reason may be that the complex canopy structure and rugged topography in natural forests lead to violation of the DHP method assumptions. Therefore, we recommend using TLS instead of DHP, in measuring leaf angle distribution in broad leaf forests. 


\section{Variation of leaf angle}

distribution in natural beech forests

*This chapter is based on: Liu, J., Skidmore, A., Wang, T., Zhu, X., Premier, J., Heurich, M., Beudert, B., Jones, S., 2019. Variation of leaf angle distribution quantified by terrestrial LiDAR in natural European beech forest. ISPRS Journal of Photogrammetry and Remote Sensing, 148, 208-220. 


\section{Abstract}

Leaf inclination angle and leaf angle distribution (LAD) are important plant structural traits, influencing the flux of radiation, carbon and water. Although LAD may vary spatially and temporally, its variation is often neglected in ecological models, due to difficulty in quantification.

In this study, terrestrial LiDAR (TLS) was used to quantify the LAD variation in natural European beech (Fagus sylvatica) forests. After extracting leaf points and reconstructing leaf surface, leaf inclination angle was calculated automatically. The mapping accuracy when discriminating between leaves and woody elements was very high across all beech stands (overall accuracy $=87.59 \%$ ). The calculation accuracy of leaf angles was evaluated using simulated point clouds and proved accurate generally $\left(R^{2}=0.88, p<0.001\right.$, RMSE $=8.37^{\circ}$, nRMSE $\left.=0.16\right)$. Then the mean $\left(\theta_{\text {mean }}\right)$, mode $\left(\theta_{\text {mode }}\right)$, and skewness of LAD were calculated to quantify the $\mathrm{LAD}$ variation.

Moderate variation of LAD was found in different successional status stands ( $\theta_{\text {mean }} \in\left(37^{\circ}, 46^{\circ}\right), \theta_{\text {mode }} \in\left[17^{\circ}, 43^{\circ}\right]$, skewness $\left.\in[0.07,0.48]\right)$. Rather than the previously assumed spherical distribution or reported planophile distribution, here we find that LAD tended towards a uniform distribution in young and medium stands, and a planophile distribution in mature stands. A strong negative correlation was also found between the plot average inclination angle $\theta_{\text {mean }}$ and plot median canopy height, making it possible to estimate plot specific LAD from canopy height data. Larger variation of LAD was found on different canopy layers ( $\theta_{\text {mean }} \in$ $\left(34^{\circ}, 53^{\circ}\right), \theta_{\text {mode }} \in\left[14^{\circ}, 64^{\circ}\right]$, skewness $\left.\in(-0.3,0.7)\right)$. Beech leaves grow more vertically in the top layer, while more obliquely or horizontally in the middle and bottom layer.

LAD variation quantified by TLS can be used to improve leaf area index (LAI) mapping and canopy photosynthesis modelling. 


\section{Introduction}

Leaf angle is an important plant structural trait. It influences light interception and radiation scattering in the canopy, as well as the flux of carbon and water (Weiss et al., 2004). Therefore, it has been used as a parameter in canopy photosynthesis modelling (Van der Tol et al., 2009), rainfall interception modelling (Xiao et al., 2000), and leaf area index (LAI) estimation. For individual leaves, leaf angle consists of leaf inclination angle and leaf azimuth angle. For the whole canopy, leaf angle distribution (LAD) is used to describe the probability of all leaves orientating at different directions. Usually a uniform azimuth direction can be assumed for most species (Ross, 1981; Falster and Westoby, 2003).

Due to measurement challenges, LAD is usually simplified using predefined mathematical functions, without considering its variation (Welles, 1990; Richardson et al., 2009; Tang et al., 2014a). Six commonly used functions are depicted in Fig. 3.1. Planophile canopies are dominated by horizontal leaves, while erectophile canopies by vertical leaves (Lemeur and Blad, 1975). The spherical distribution (de Wit, 1965) is the most widely used due to its simplicity in calculating the leaf projection function value (approximated as 0.5 in any direction).

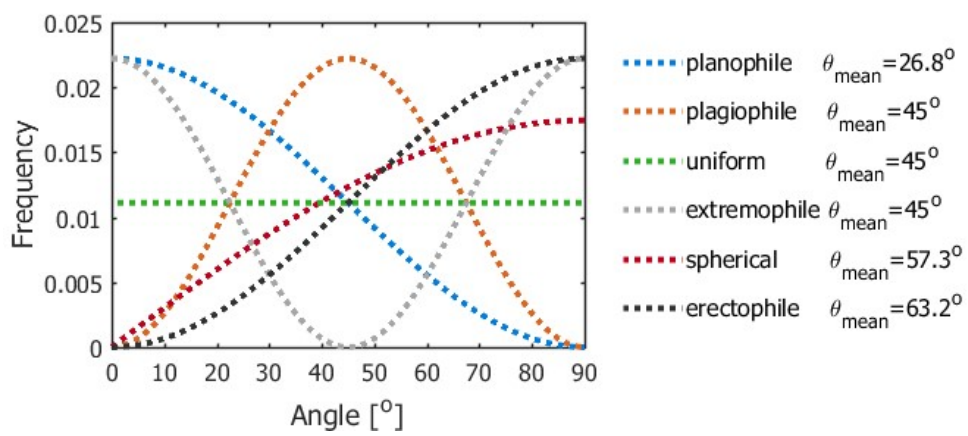

Figure 3.1: Six predefined mathematical functions used to approximate leaf angle distribution and the corresponding average leaf inclination angle $\left(\theta_{\text {mean }}\right)$

However, such simplification fails to consider the variation of LAD. In reality, LAD may vary for different species of plants (Pisek et al., 2013). Even for the same species, LAD may also exhibit a spatial and temporal variability. LAD was found to vary in different canopy layers in a tropical 
forest (Wirth et al., 2001), and several temperate deciduous trees (Raabe et al., 2015). LAD may also change with light exposure (McMillen and McClendon, 1979; Utsugi et al., 2006), time of the day (Shell et al., 1974) and season (Hosoi and Omasa, 2009; Raabe et al., 2015). Due to oversimplification, LAD has become one of the most poorly constrained radiative transfer model parameters (Ollinger, 2011). There is great potential to improve canopy photosynthesis modelling and LAI mapping if the $\mathrm{LAD}$ variation can be quantified.

One strategy to measure LAD is the direct geometrical approach. The basic principle is to obtain a representative description of the whole canopy by observations on individual leaf (Norman and Campbell, 1989), for example using compass and inclinometers (Ross, 1981). But direct contact often leads to disturbance of the leaves (Zheng and Moskal, 2012). The spatial coordinate apparatus (Lang, 1973) method was proposed to avoid direct contact, but the required number of leaves takes a large logistical effort to measure in forests. Consequently, the levelled digital canopy photography method has been introduced (Ryu et al., 2010; Pisek et al., 2011). In this method, the authors first took several photos around the canopy at different heights. Then leaves were visually identified from each photo and each leaf angle was calculated using image processing (Pisek et al., 2011). Although this method is robust and low-cost, it involves substantial user interaction when identifying individual leaves. In addition, taking photos for trees higher than $2 \mathrm{~m}$ is very difficult in natural forests.

Another strategy to estimate LAD is the indirect radiometric approach. This approach yields a statistical estimate of LAD on plot level, rather than measuring the orientation of an individual leaf (Biskup et al., 2007). The basic principle is to record how radiation is attenuated by the canopy in several zenith directions, then one can invert the Beer's law for radiation interception to infer LAD (Norman and Campbell, 1989; Chen et al., 1991). However, this method has two main shortcomings. First, it cannot distinguish leaf and woody material. What was retrieved is the plant angle distribution rather than LAD. Second, the radiometric method makes assumptions (flat topography and homogeneous tree height) which may not hold in heterogeneous natural forests.

With the development of close range remote sensing, efforts were made 
using three dimensional (3D) point cloud data to quantify canopy structure (Coops et al., 2007; Hancock et al., 2014). Magnetic 3D digitizer (Sinoquet et al., 1998; Falster and Westoby, 2003), film-based stereo photogrammetry (Ivanov et al., 1995) and digital stereo imaging (Biskup et al., 2007) have been used to obtain 3D reconstructions of plants. But the drawback of these methods is their restricted usage to the outer canopy of small stands, usually an area of a few square meters (Muller-Linow et al., 2015). When combined with unmanned aerial vehicles (UAV), it can sample larger areas at stand scale. However, UAV-mounted cameras are vulnerable to lens distortion and image noise (McNeil et al., 2016).

From the 1990s, terrestrial LiDAR (TLS) has received increasing attention in vegetation surveys due to its capability to capture unprecedented detail of plant 3D structure, from individual tree to plot scale (Liang et al., 2018). With high pulse frequency and small beam divergence, tree trunks, branches, and even leaves can be easily recorded (Eitel et al., 2010). TLS data was used to estimate LAD using indirect radiometric inversion (Zhao et al., 2015). TLS data was also used to visually delineate individual leaf (Béland et al., 2011), and automatically reconstruct leaf surface and normal vectors at individual tree scale (Zheng and Moskal, 2012). However, there was no leaf size constraint in the leaf reconstruction. Instead, a fixed number of 6 neighboring points was used to form each leaf surface (Zheng and Moskal, 2012). This may be problematic for upper canopy layers when point density is low, making the distance among the 6 points much larger than the size of an individual leaf. Recently, a rapid LAD estimation method was developed based on triangulation of TLS point clouds (Bailey and Mahaffee, 2017). This method demonstrated good accuracy for an isolated tree and a vineyard. However, the calculated LAD should be more precisely named plant angle distribution, as leaf and woody material were not differentiated.

To the best of our knowledge, the leaf angle distribution (LAD) variation has only been quantified at individual canopy level by manual measurement (Wirth et al., 2001; Holder, 2012), levelled digital canopy photography methods (Raabe et al., 2015), or digital stereo imaging (Muller-Linow et al., 2015). All these methods are difficult to implement in natural forests. The objective of this research is therefore to use terrestrial LiDAR to explore whether there is LAD variation at different 
canopy layers and across different succession stands in European beech (Fagus sylvatica) forests.

\section{Research data}

\subsection{Study area and plot distribution}

The study area is the Bavarian Forest National Park, located in southeastern Germany. It is a low mountain range forest ecosystem in Central Europe, with elevation ranging from $650 \mathrm{~m}$ to $1453 \mathrm{~m}$. It is located in the temperate climate zone and is subject to maritime and continental influences (Bässler et al., 2008). Mean annual precipitation is between 830 and $2230 \mathrm{~mm}$ depending on altitude. Dominant tree species are Norway spruce (Picea abies) (67\%) and European beech (Fagus sylvatica) (24.5\%) (Cailleret et al., 2014). In this research, European beech is selected due to its broadleaf feature, as well as its wide distribution in Western and Central Europe. Beech trees normally grow to $30-35 \mathrm{~m}$ (up to $50 \mathrm{~m}$ in optimal conditions) tall. A 15-year-old sapling stands about $4 \mathrm{~m}$. The bark of European beech is smooth as seen in Fig $3.2 \mathrm{~b}$ ). The leaves are elliptical without any lobes and have a short stalk, as seen in Fig 3.2 a). Leaf size ranges in $25-40 \mathrm{~cm}^{2}$, with $5-10 \mathrm{~cm}$ long and $3-7 \mathrm{~cm}$ wide (Barna, 2004). European beech is a highly shade-tolerant species, which can regenerate naturally in continuous cover. In Central Europe, it is the most abundant broad leaf forest tree, because of its physiological tolerance and competitiveness (Ellenberg and Leuschner, 2010).

In total, 36 European beech plots were selected as shown in Fig 3.3. They covered a wide range of stand structures and were further categorized into "young, medium, mature" stands using ancillary land cover classification data (Silveyra Gonzalez et al., 2018) and canopy height information. The structural information, including the median and standard deviation of canopy height, was extracted from airborne laser scanning data in 2016. More details can be seen in Table 3.1. 

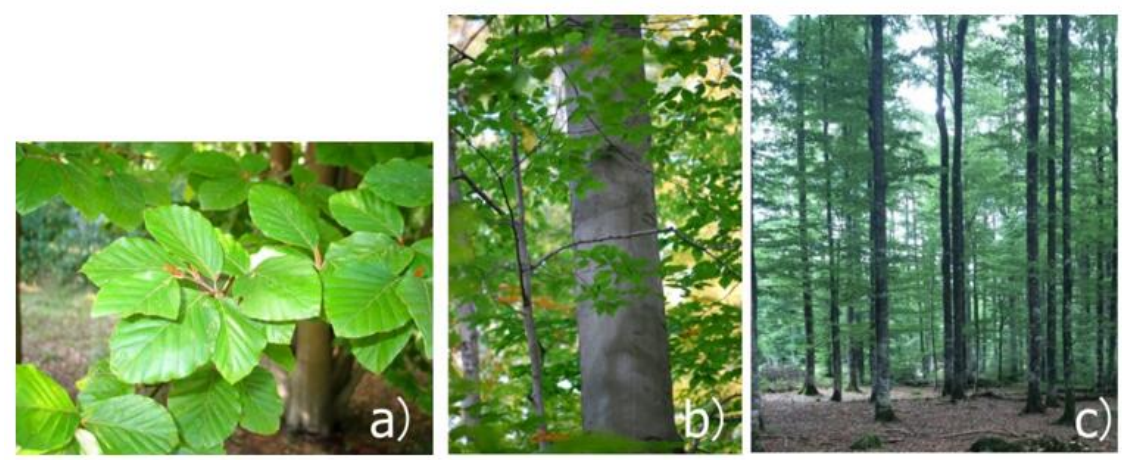

Figure 3.2: European Beech (Fagus sylvatica) in the Bavarian Forest National Park. a) leaves; b) trunk and branch; c) a mature plot

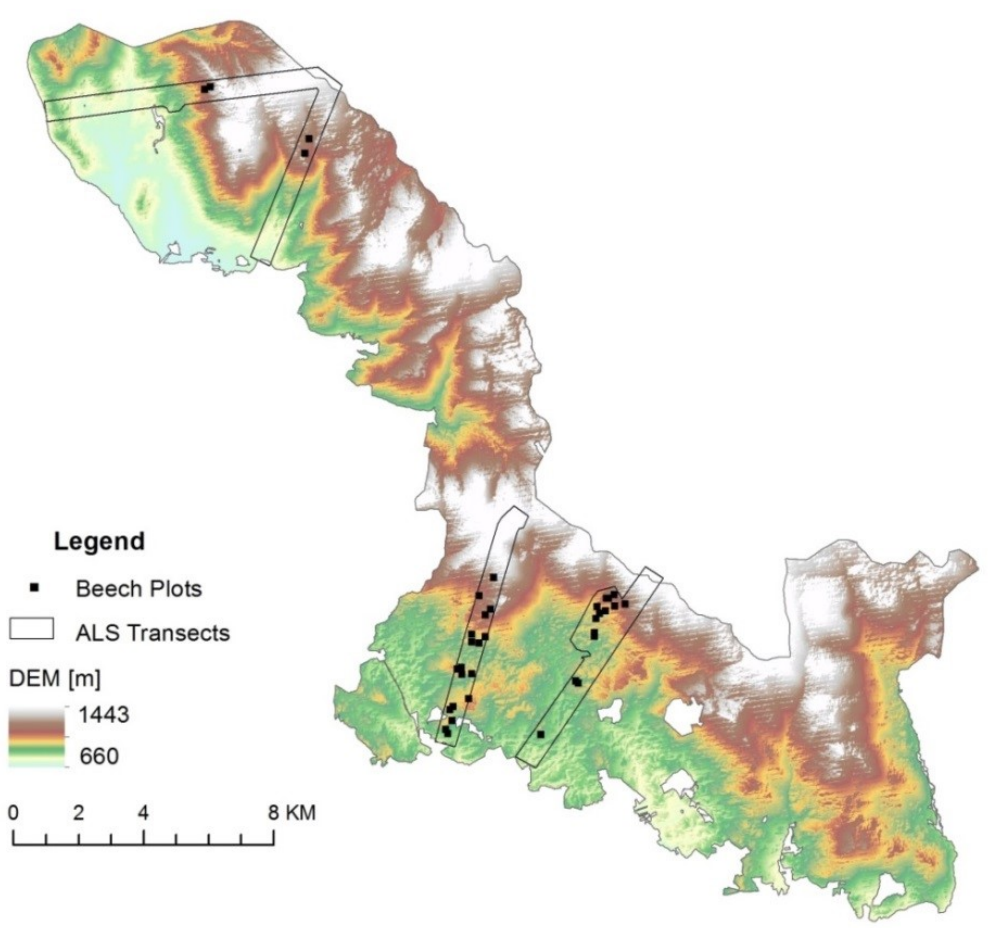

Figure 3.3: The distribution of 36 European beech plots in the Bavarian Forest National Park 
Table 3.1: Basic information of the 36 European beech plots

\begin{tabular}{|c|c|c|c|c|c|}
\hline Plot Name & MedianH [m] & StdH [m] & Elevation [m] & Slope $\left[{ }^{\circ}\right]$ & $\mathrm{N} / \mathrm{ha}$ \\
\hline B01 & 33.6 & 11.5 & 808.3 & 9.2 & 280 \\
\hline B02 & 25.6 & 7 & 822.6 & 4.4 & 380 \\
\hline B03 & 23.4 & 1.7 & 844.6 & 8.9 & 540 \\
\hline B04 & 22.6 & 9.9 & 862.6 & 8.7 & 460 \\
\hline B05 & 25.6 & 4.8 & 836.2 & 6.1 & 420 \\
\hline B06 & 22.2 & 9.9 & 839.4 & 4.9 & 380 \\
\hline B07 & 25.7 & 3.7 & 853.5 & 9.8 & 280 \\
\hline B08 & 25.7 & 7.2 & 847.7 & 14.2 & 280 \\
\hline B09 & 8 & 8.6 & 829.7 & 7.7 & 540 \\
\hline B10 & 16 & 6.8 & 869.2 & 8.8 & 1840 \\
\hline B11 & 27.5 & 4.5 & 841.5 & 5.7 & 640 \\
\hline B12 & 26.8 & 5.8 & 851 & 9 & 220 \\
\hline B13 & 21.6 & 4 & 915.9 & 14.5 & 820 \\
\hline B14 & 20.2 & 7.3 & 964.2 & 11 & 320 \\
\hline B15 & 20.8 & 7.7 & 1133.9 & 17.6 & 1140 \\
\hline B16 & 13 & 3.2 & 990.9 & 11.8 & 2200 \\
\hline B17 & 24.4 & 2.5 & 1019.7 & 11 & 460 \\
\hline B18 & 13.6 & 5.1 & 781.9 & 5.3 & 980 \\
\hline B19 & 23.3 & 3.2 & 1030.4 & 16.1 & 480 \\
\hline B20 & 8.2 & 4.6 & 792.9 & 5.7 & 520 \\
\hline B21 & 17.1 & 5.5 & 826.8 & 7.5 & 420 \\
\hline B22 & 11 & 8.7 & 892.4 & 9.7 & 260 \\
\hline B23 & 21.3 & 8 & 962.3 & 14.2 & 240 \\
\hline B24 & 18.6 & 5.1 & 1043.9 & 18.4 & 1140 \\
\hline B25 & 22 & 5.7 & 972.4 & 8.5 & 540 \\
\hline B26 & 8.3 & 5 & 912.4 & 11.5 & 500 \\
\hline $\mathrm{B} 27$ & 4.2 & 6.1 & 866.6 & 8.3 & 240 \\
\hline B28 & 6.9 & 7.4 & 796.4 & 6.4 & 400 \\
\hline B29 & 21.3 & 3 & 911.7 & 7.7 & 480 \\
\hline B30 & 31.1 & 1.5 & 775.7 & 13.6 & 480 \\
\hline B31 & 27.3 & 1.7 & 981.8 & 9.3 & 520 \\
\hline B32 & 26.2 & 1.9 & 834.1 & 4.1 & 420 \\
\hline B33 & 21.4 & 1.6 & 1082 & 9.2 & 960 \\
\hline B34 & 18.4 & 8.2 & 1096.3 & 15.5 & 660 \\
\hline B35 & 18.5 & 12.7 & 982.1 & 18 & 360 \\
\hline B36 & 11.6 & 10.6 & 1050.9 & 18 & 320 \\
\hline
\end{tabular}

\subsection{Data collection}

From 17-July to 9-August in 2017, 36 beech plots were visited during leaf-on conditions. A Riegl VZ-400 TLS was used to scan each plot. The scanner employs a laser (wavelength $1550 \mathrm{~nm}$ ) with a beam divergence of $0.3 \mathrm{mrad}$ and a range accuracy of $5 \mathrm{~mm}$. The footprint diameter is $0.15 \mathrm{~cm}$ at a distance of $5 \mathrm{~m}, 1.05 \mathrm{~cm}$ at a distance of $35 \mathrm{~m}$. The measurement range is up to $600 \mathrm{~m}$. The angular step was set to $0.04^{\circ}$ for the fieldwork. 
One center and three triangular scan positions were used in each plot, to reduce occlusion and increase point density. To achieve co-registration of the four scans, 12-18 retro-reflective targets were placed as control points. In total, it took 1-2 hours to finish all scanning in a plot.

In addition to TLS data, four transects in the park were also scanned by airborne laser scanning (ALS) in August 2016, seen in Fig 3.3. The sensor used was Riegl LMS-Q680-i, operating at a wavelength of $1550 \mathrm{~nm}$, with a beam divergence of $0.5 \mathrm{mrad}$. The flying altitude was approximately $300 \mathrm{~m}$ above ground. The average point density for each flight line was 70 points $/ \mathrm{m}^{2}$. The ALS data was used to calculate basic plot structure metrics including canopy height model (CHM), the median and standard deviation of canopy height (details in Table 3.1).

\section{Method}

\subsection{Preprocessing}

First, the four TLS scans were co-registered and merged into one for each plot, to maximize point density, using the Riscan Pro software (http://www.riegl.com). The average registration error was 3-8 $\mathrm{mm}$. Then, a point cloud with a radius of $15 \mathrm{~m}$ was clipped. Each point consisted of multiple attributes, including the Cartesian coordinates (x, y, z), laser shot direction (azimuth and zenith angle), target distance, amplitude of the echo, GPS timestamp, target surface relative reflectance, pulse shape deviation, etc. Filtering was conducted to remove noisy points, based on the pulse shape deviation value. Pulse shape deviation may be interpreted as a measure of the reliability of the range measurement (Pfennigbauer and Ullrich, 2010). The overall quality of the point cloud can be improved by setting up a maximum allowed deviation value. In this research, all points with deviation above 20 were eliminated. This threshold was based on suggestions from previous research (Pfennigbauer and Ullrich, 2010; Greaves et al., 2015). After noise filtering, ground returns were identified and the local height of each point was calculated using LAStools software (Isenburg, 2012). All points below $1.5 \mathrm{~m}$ such as ground and grass were removed from subsequent analysis. 


\subsection{Differentiating between leaf and woody material}

TLS has shown promising results in differentiating leaf and woody material (Beland et al., 2014; Zhu et al., 2018a; Li et al., 2018b). In this research, the point cloud was classified into leaf or woody points, thus eliminating the effect of woody material to retrieve LAD rather than PAD. The classification followed the method using both radiometric and geometric features (Zhu et al., 2018a). The significant difference in reflectivity between leaf and woody material at the $1550 \mathrm{~nm}$ wavelength forms the basis to use radiometric features. The bark has high reflectance, while leaves have low reflectance due to water absorption. Geometric features of leaf and woody material are also different. Leaves have planar shape, while woody material is more likely to have linear shape. A list of selected features (seen in Table 3.2) was calculated for each point, for the detailed equation one can refer to (Demantke et al., 2011; Zhu et al., 2018a).

Table 3.2: Radiometric and geometric features used to differentiate leaf and woody points

\begin{tabular}{|c|c|c|}
\hline Type & Feature & Description \\
\hline \multirow{4}{*}{$\begin{array}{l}\text { Radiometric } \\
\text { features }\end{array}$} & Ref & calibrated relative reflectance \\
\hline & $\operatorname{Ref}_{\text {mean }}$ & mean Ref of the local points \\
\hline & $\operatorname{Ref}_{\text {std }}$ & standard deviation of Ref of the local points \\
\hline & Dev & pulse shape deviation \\
\hline \multirow{5}{*}{$\begin{array}{l}\text { Geometric } \\
\text { features }\end{array}$} & $\alpha_{1 \mathrm{D}}$ & the likelihood that the shape of the local points is \\
\hline & $\alpha_{2 \mathrm{D}}$ & $\begin{array}{l}\text { the likelihood that the shape of the local points is } \\
\text { planar }\end{array}$ \\
\hline & $\alpha_{3 \mathrm{D}}$ & $\begin{array}{l}\text { the likelihood that the shape of the local points is } \\
\text { random }\end{array}$ \\
\hline & $Z_{\text {diff }}$ & $\begin{array}{l}\text { range of maximum and minimum height value of } \\
\text { the local points }\end{array}$ \\
\hline & $Z_{\text {std }}$ & standard deviation of height in the local points \\
\hline
\end{tabular}

After feature calculation, the Support Vector Machine (SVM) classifier was used to differentiate leaf and woody points. SVM is a supervised non-parametric statistical learning technique. It shows to achieve good results even with small training datasets in high dimensional feature space (Melgani and Bruzzone, 2004), often producing higher classification accuracy than other methods (Foody and Mathur, 2004). In this research, training samples were manually selected from one plot at different layers (top, middle and bottom layer). Then the points were labeled with a class 
of either "leaf" or "woody" based on visual interpretation. These training samples (357 leaf points and 359 woody points) were used to build the SVM model, and applied to all 36 plots. After initial classification, a post filtering was conducted on all detected leaf points. If the majority of the neighboring points of a leaf point are woody points, then the class label of this point was changed to "woody". Classification accuracy was evaluated in all 36 plots. For each plot, the point cloud was first partitioned into 12 sector cylinders $\left(0^{\circ}, 30^{\circ}, \ldots, 360^{\circ}\right)$, and sliced into 10 vertical layers $(1 / 10,2 / 10, \ldots, 1)$. Then a random point was selected from each of these $120 \mathrm{sub}$ point clouds. To define the true class label of each test sample point, all its neighboring points within a $50 \mathrm{~cm}$ radius spheroid were displayed. With the help of contextual information, (i.e. the point locates in a branch or a leaf), the class label (wood or leaf) of this point could be determined through visual interpretation. The classification accuracy was then calculated.

\subsection{Reconstructing surface and calculating normal vector}

After classification, individual leaf surfaces were reconstructed on the leafy point cloud through plane fitting constrained by leaf size. Let $S=$ $p_{i}(\mathrm{x}, \mathrm{y}, \mathrm{z}), \mathrm{i} \in\left[1, N_{\text {total }}\right]$ be the point cloud of the plot in the Cartesian coordinate system, $N_{\text {total }}$ is the number of all points. For point $p_{k}$, its neighboring points $S^{\prime}=\left(p_{1}, p_{2}, \ldots, p_{n}\right)$ could be identified by searching all points in $S$ that are within a distance $L_{\max }$ of point $p_{k}$. If the number of neighboring points, i.e. $n$, is greater than 5 , these points were considered to form a leaf surface. The normal vector of $S^{\prime}$ was calculated through principal component analysis. The normal vector direction is the same as the direction of the eigenvector with the minimum eigenvalue. If $n$ is smaller or equal to 5 , point $p_{k}$ was considered as an isolated point, and eliminated from subsequent analysis. In theory, within the $L_{\max }$ radius, 3 points can form a plane. However, to avoid uncertainty caused by noise points, we used 5 neighboring points (in total 6 points) to do the plane fitting. For more discussion on this, one can refer to a previous study (Hoppe et al., 1992). It should be noted that in this method, no differentiation was made between leaves having an adaxial sky facing surface or abaxial sky facing surface, thus all leaf inclination angles are 
positive.

The radius of this neighborhood distance $L_{\max }$, should be constrained by the leaf size. If the value of $L_{\max }$ is very low, many points were processed as isolated points and eliminated, since their neighboring points are beyond the distance of $L_{\max }$. However, if the value of $L_{\max }$ is very high, points from two adjacent leaves may be merged into one neighborhood $S$. In a previous research, $L_{\max }$ was set to be $5 \mathrm{~cm}$ (Bailey and Mahaffee, 2017). In this research, after considering the leaf size of European beech (as mentioned in section 2.1, usually $5-10 \mathrm{~cm}$ long and 3-7 cm wide), $L_{\max }$ was set to be $4 \mathrm{~cm}$.

\subsection{Validating accuracy of the leaf angle calculation method}

In order to evaluate the accuracy of the leaf angle calculation method, we generated a simulated dataset, where the location and the true angle of each leaf was known, similarly to techniques used in previous research (Bailey and Mahaffee, 2017; Li et al., 2018a). First, the 3D models of two synthetic beech trees, one young ( $3 \mathrm{~m}$ high, 1122 leaves) and one mature (30m high, 21534 leaves) were constructed using the open source software Arbaro (http://arbaro.sourceforge.net/). The LiDAR simulator HELIOS (Bechtold and Höfle, 2016) was employed to "scan" the beech tree, using the same settings as used in the fieldwork ( $0.3 \mathrm{mrad}$ beam divergence, $0.04^{\circ}$ angular step, $7.5 \mathrm{~m}$ distance away, 3 scan positions). For the mature tree, titled $\left(90^{\circ}\right)$ scans were used to ensure coverage on the canopy top. Leaf angles were calculated using our proposed method from the simulated TLS point cloud. The calculation accuracy was evaluated in the "leaf-wise" way, by comparing all leaf angles estimated from the simulated TLS point cloud with true leaf angles from the 3D models. The coefficient of determination $\left(R^{2}\right)$, the root mean square error (RMSE), and the normalized RMSE (nRMSE) were used to evaluate the performance of the method.

\subsection{Statistical analysis}

LAD was retrieved through calculating the histogram of the inclination angles of all reconstructed leaf surfaces, as the frequency distribution 
from $0^{\circ}$ to $90^{\circ}$ with $1^{\circ}$ bin width. Four statistical parameters of the leaf angle distribution (LAD) were also calculated, including the median angle $\left(\theta_{\text {median }}\right)$, the average incliantion angle $\left(\theta_{\text {mean }}\right)$, the most frequent incliantion angle $\left(\theta_{\text {mode }}\right)$, and skewness of LAD (skewness). In addition, each LAD was classified into one of the six categorical (planophile, plagiophile, uniform, spherical, erectophile, extremophile) LAD functions (de Wit, 1965) as detailed in Fig 3.1. This was done by quantifying the similarity of plot LAD with the six pre-defined LAD functions (Pisek et al., 2013) through the following three metrics,

$$
\begin{aligned}
& \chi_{1}=\sum_{\theta=0}^{90}\left|f(\theta)-f_{\text {deWit }}(\theta)\right| \\
& \chi_{2}=\sqrt{\frac{\sum_{\theta=0}^{90}\left(f(\theta)-f_{\text {deWit }}(\theta)\right)^{2}}{90}} \\
& \chi_{3}=\frac{\left|f(\theta) \cap f_{\text {deWit }}(\theta)\right|}{\left|f(\theta) \cup f_{\text {deWit }}(\theta)\right|}=\frac{\sum_{\theta=0}^{90} \min \left(f(\theta), f_{\text {deWit }}(\theta)\right)}{\sum_{\theta=0}^{90} \max \left(f(\theta), f_{\text {deWit }}(\theta)\right)}
\end{aligned}
$$

The pre-defined LAD which had the lowest $\chi_{1}, \chi_{2}$, or highest $\chi_{3}$ will be voted as similar to the plot LAD. The LAD type, which received the highest count of votes, was chosen as the classification result for the plot LAD. In order to explore the variation of LAD on different height levels in a canopy, each plot was divided into 3 layers according to the local height of each point. Let $H_{\max }$ be the maximum canopy height. Points with greater than $80 \% H_{\max }$ were treated as the top layer. Points at $80 \%$ to $20 \% H_{\max }$ were regarded as the middle layer. Points below $20 \%$ $H_{\text {max }}$ were used as the bottom layer. The above-mentioned analyses were conducted on each layer of each plot. In order to explore the variation of LAD across stands, the above-mentioned analyses were conducted for each plot. The correlation coefficient was calculated between $\theta_{\text {mean }}$ and the plot median canopy height, to explore the relationship between plot LAD and plot successional status. A positive close to 1 correlation coefficient would suggest a strong positive correlation between the $\theta_{\text {mean }}$ and the stand successional status. 


\section{Results}

\subsection{Accuracy of leaf angle calculation}

From the simulated dataset, we compared the TLS estimated leaf angles with true leaf angles directly read from the synthetic beech tree model. Through this, we can evaluate the accuracy of our proposed method. From the results in Fig 3.4, for the synthetic young beech tree $(3 \mathrm{~m}$ high, with 1122 leaves), the proposed method works very well $\left(R^{2}=0.88\right.$, $\mathrm{RMSE}=8.37^{\circ}$, nRMSE $\left.=0.16\right)$. For the synthetic mature beech tree $(30 \mathrm{~m}$ high, with 21534 leaves), although there are some leaves with larger estimation errors for leaf angle, the overall accuracy remains high $\left(R^{2}=0.83\right.$, RMSE $=9.29^{\circ}$, nRMSE $=0.20$ ).
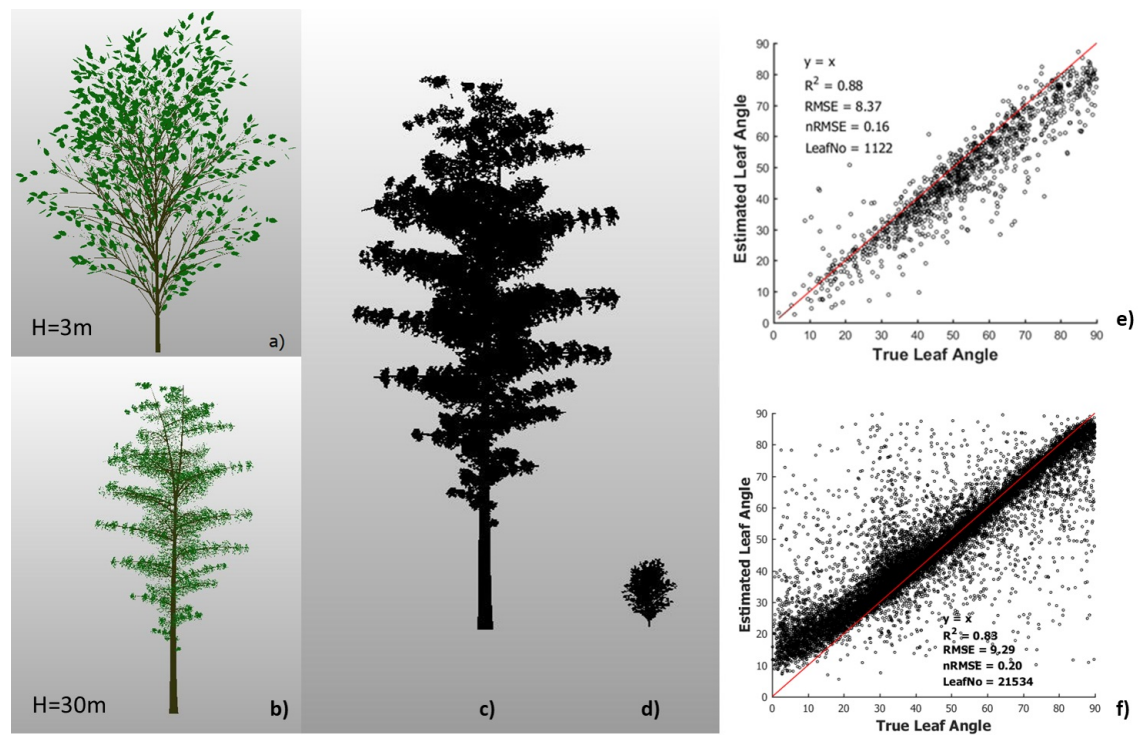

Figure 3.4: Results of the leaf angle calculation accuracy using simulated dataset: a) d) the 3D models of two synthetic beech trees; b) e) the simulated TLS point clouds of the beech trees; c) f) true leaf angles and leaf angles estimated using the proposed method 


\subsection{Results of differentiating leaf and woody materials}

In this study, the overall classification accuracy when differentiating leaf and woody materials is $87.59 \%$ across 36 beech plots, with the accuracy of each plot ranging from $78.26 \%$ to $94.32 \%$ (details in Table 3.3)). An example of the classification result is displayed in Fig 3.5. Large tree trunks and smaller branches could be accurately detected. At $8 \mathrm{~m}$ level, most leaves were covered by dense points and were accurately detected, shown in Fig 3.5 c). However, small twigs were often misclassified as leaves. Similar results occurred at the $16 \mathrm{~m}$ level in Fig 3.5 d). Although the point density was not as high as at lower height levels, tree trunks, branches, and leaves could still be differentiated. But fine twigs were again misclassified as leaves.

Table 3.3: The overall accuracy (OA) of classification between leaf and woody materials in the 36 plots

\begin{tabular}{llllll}
\hline Plot Name & OA[\%] & Plot Name & OA[\%] & Plot Name & OA[\%] \\
\hline B01 & 83.72 & B13 & 90.32 & B25 & 86.32 \\
B02 & 89.54 & B14 & 94.32 & B26 & 82.65 \\
B03 & 92.78 & B15 & 90.63 & B27 & 82.42 \\
B04 & 90.11 & B16 & 88.54 & B28 & 87.64 \\
B05 & 92.71 & B17 & 91.49 & B29 & 80.46 \\
B06 & 93.68 & B18 & 83.52 & B30 & 88.37 \\
B07 & 93.33 & B19 & 90.32 & B31 & 85.71 \\
B08 & 91.21 & B20 & 82.29 & B32 & 88.64 \\
B09 & 87.95 & B21 & 85.06 & B33 & 89.25 \\
B10 & 80.22 & B22 & 86.02 & B34 & 78.26 \\
B11 & 88.04 & B23 & 86.17 & B35 & 85.56 \\
B12 & 90.53 & B24 & 82.11 & B36 & 93.67 \\
\hline
\end{tabular}



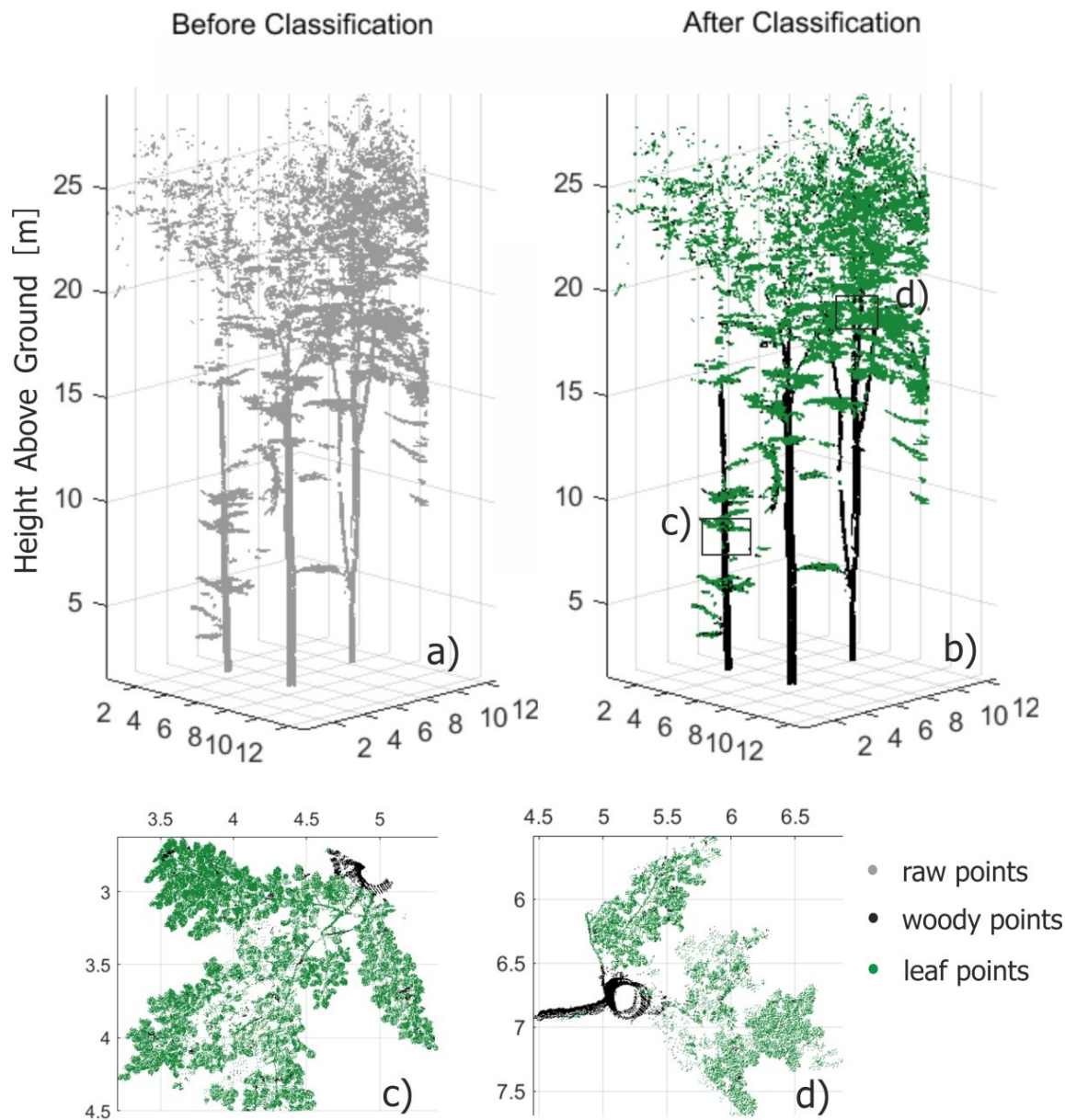

Figure 3.5: Results of differentiating leaf and woody materials in part of a mature beech plot B32: a) before and b) after classification; detailed results at c) $8 \mathrm{~m}$ and d) $16 \mathrm{~m}$ above ground 


\subsection{LAD variation on different canopy layers}

An example of the 3D distribution of leaf inclination angle in a mature plot is shown in Fig 3.6. In the top layer, many leaves are displayed as red and yellow colors $\left(50^{\circ}-80^{\circ}\right)$, indicating a more vertical growing direction. But in the middle layer and bottom layer, most leaves display blue or cyan colors $\left(10^{\circ}-40^{\circ}\right)$, indicating a more horizontal and lateral growing direction. From statistical parameters and LAD classifications in Fig 3.6 g), LAD in the top layer is most similar to the uniform distribution, with largest $\theta_{\text {median }}, \theta_{\text {mean }}, \theta_{\text {mode }}$, and lowest skewness. But LAD the middle and bottom layers is most similar to the planophile distribution. The $\theta_{\text {median }}, \theta_{\text {mean }}$ and $\theta_{\text {mode }}$ decrease, while skewness rises. In general, from the top to bottom layer, the frequency of vertical leaves decreases, while the frequency of more horizontal (oblique) leaves increases.

\subsection{LAD variation at different succession stands}

The 3D distribution of leaf inclination angles in three different stands can be seen in Fig 3.7. In a young plot B27 with beech regeneration, stem density is low. Beech canopies have a spheroid rather than cylinder shape, as seen in Fig 3.7 d), with leaves orientated in various directions. In a medium plot B10, stem density becomes much higher. Leaves grow in various directions in the top layer, but grow more horizontally in the middle layer. In a mature plot B31, the vertical difference of LAD becomes more pronounced (Fig 3.7 c) and Fig 3.7 f)). 


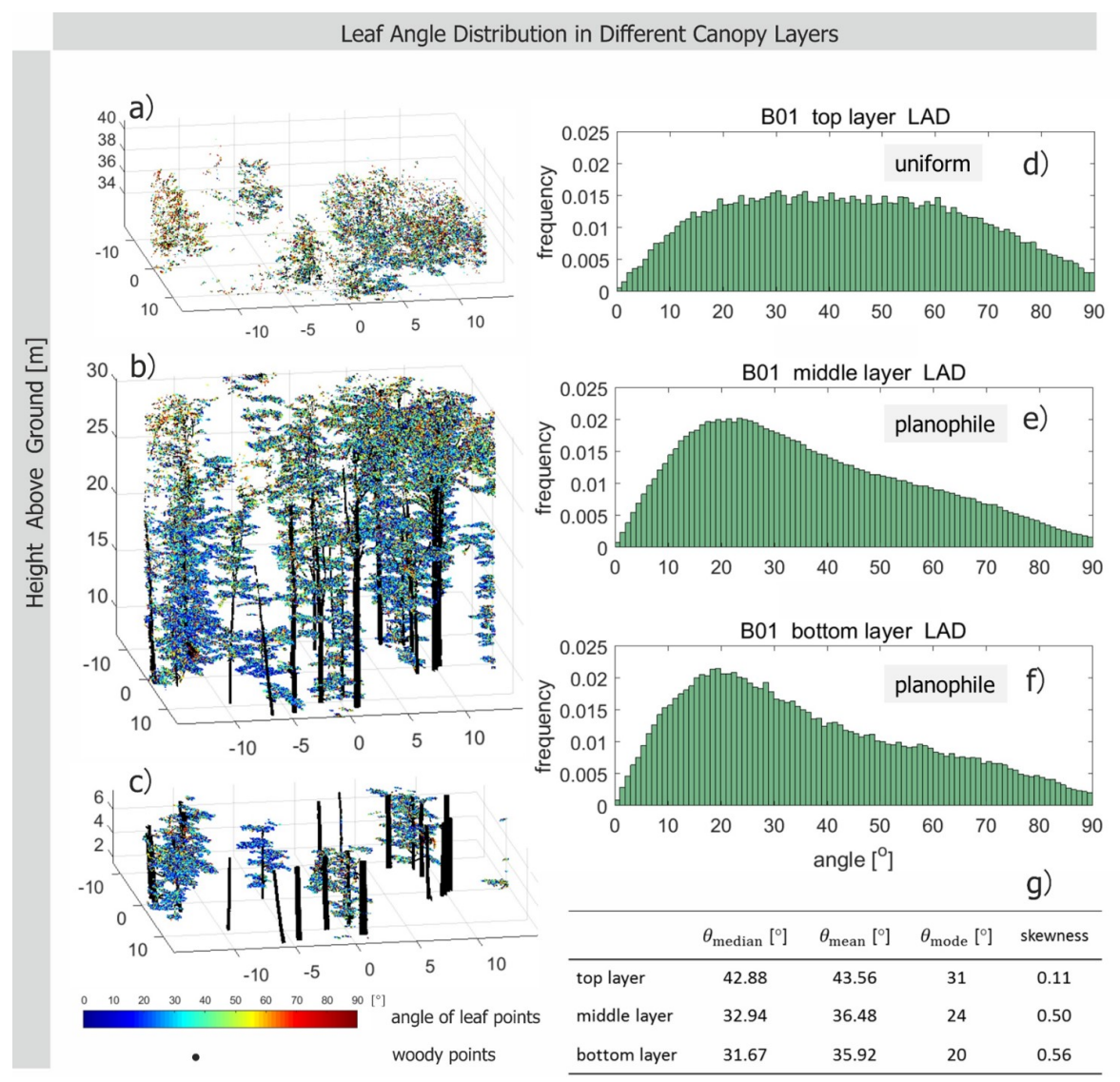

Figure 3.6: LAD at different canopy layers in the mature beech plot B01. a), b) and c) are the 3D distribution of leaf angles; d), e) and f) are the LAD histograms; g) summary of statistics 


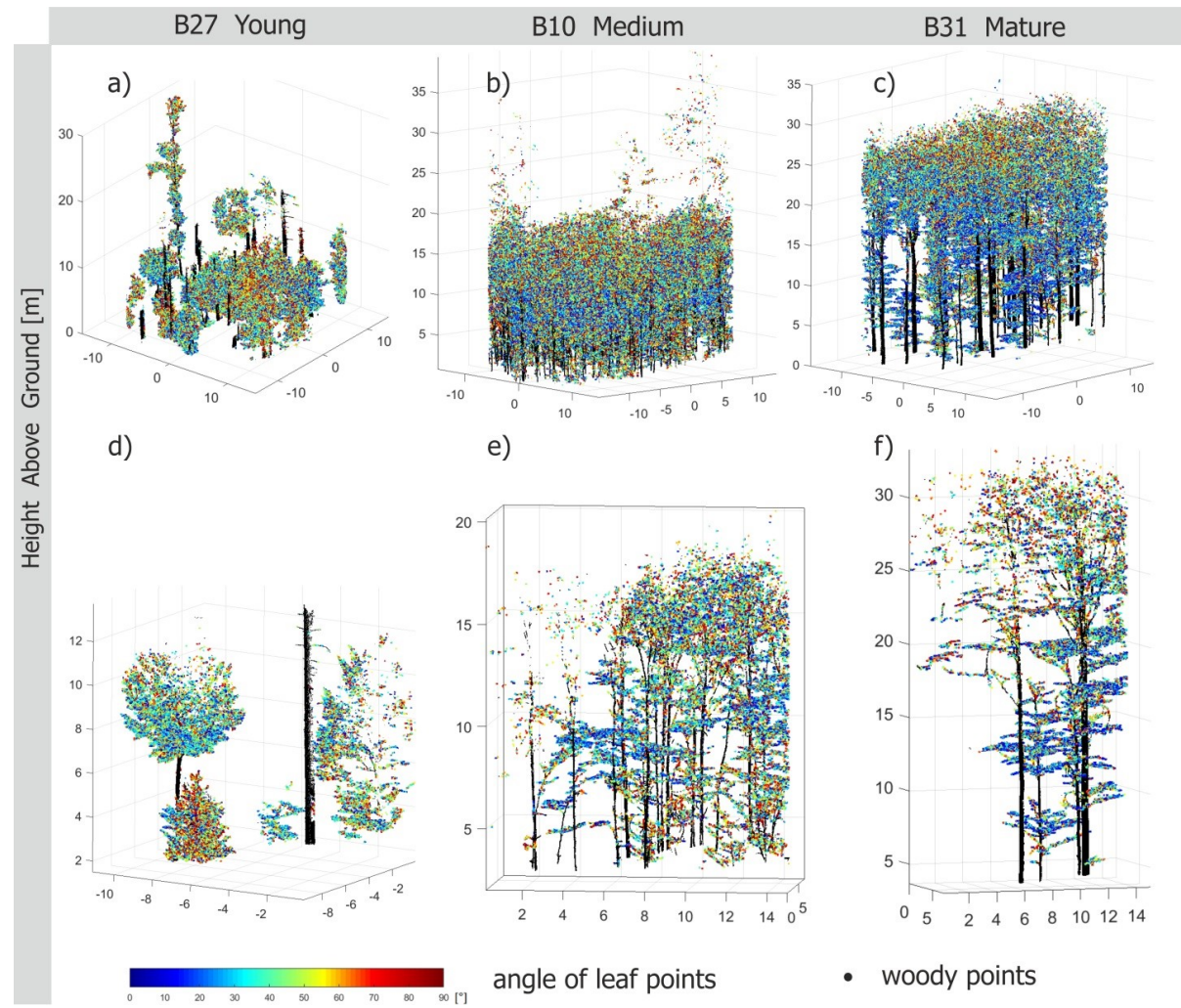

Figure 3.7: LAD on plots of different successional status. The 3D distribution of leaf angles in a) a young plot B27; b) a medium plot B10; c) a mature plot B31; d) part of B27; e) part of B10; f) part of B31 
Statistical parameters demonstrate moderate variation of LAD across these three stands (Table 3.4 and Fig 3.8). From young to mature stands, the $\theta_{\text {mean }}$ decreases about $8^{\circ}$, while $\theta_{\text {mode }}$ decreases $22^{\circ}$. The LAD of the young plot B27 and medium plot B10 is most similar to the uniform distribution, but LAD of the mature plot B31 is most similar to the planophile distribution, with a highly positive skewness of 0.42 .
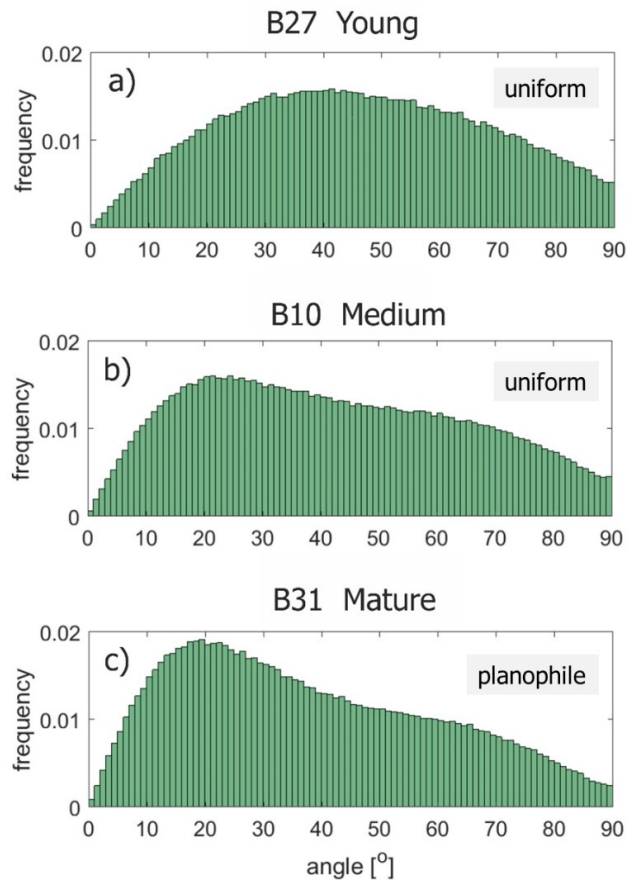

Figure 3.8: LAD of plots at different successional status: a) a young plot B27; b) a medium plot B10; c) a mature plot B31.

Table 3.4: Summary of LAD statistics across three different stands

\begin{tabular}{lcccccc}
\hline Plot & Succession Status & $\theta_{\text {median }}$ & $\theta_{\text {mean }}$ & $\theta_{\text {mode }}$ & skewness & Type \\
\hline B27 & young & 45.43 & 46.14 & 42 & 0.07 & uniform \\
B10 & medium & 40.24 & 42.28 & 25 & 0.23 & uniform \\
B31 & mature & 34.38 & 37.98 & 20 & 0.42 & planophile \\
\hline
\end{tabular}


The LAD variation across all 36 stands is depicted in Fig 3.9. The relationship between LAD and stand succession status was explored by inspecting $\theta_{\text {mean }}$ and skewness with the median canopy height in the plot $\left(\mathrm{CHM}_{\text {median }}\right)$. Interestingly, plot $\theta_{\text {mean }}$ is strongly negatively related to $\mathrm{CHM}_{\text {median }}(r=-0.70, p<0.001)$, while skewness of LAD is moderately positively related to $\mathrm{CHM}_{\text {median }}(r=0.64, p<0.001)$. In young and medium plots $\left(\mathrm{CHM}_{\text {median }} \in(4,20) \mathrm{m}\right)$, $\mathrm{LAD}$ is most similar to the uniform distribution. However in mature plots $\left(\mathrm{CHM}_{\text {median }}>20 \mathrm{~m}\right)$, LAD is most similar to the planophile distribution. Among all 36 plots, the difference between minimum and maximum $\theta_{\text {mean }}$ is moderate $\left(10^{\circ}\right.$, from $36.91^{\circ}$ to $46.14^{\circ}$ ). It is also worth noted that the LAD in all plots are quite different from the spherical distribution.

\section{LAD across 36 Beech Stands}

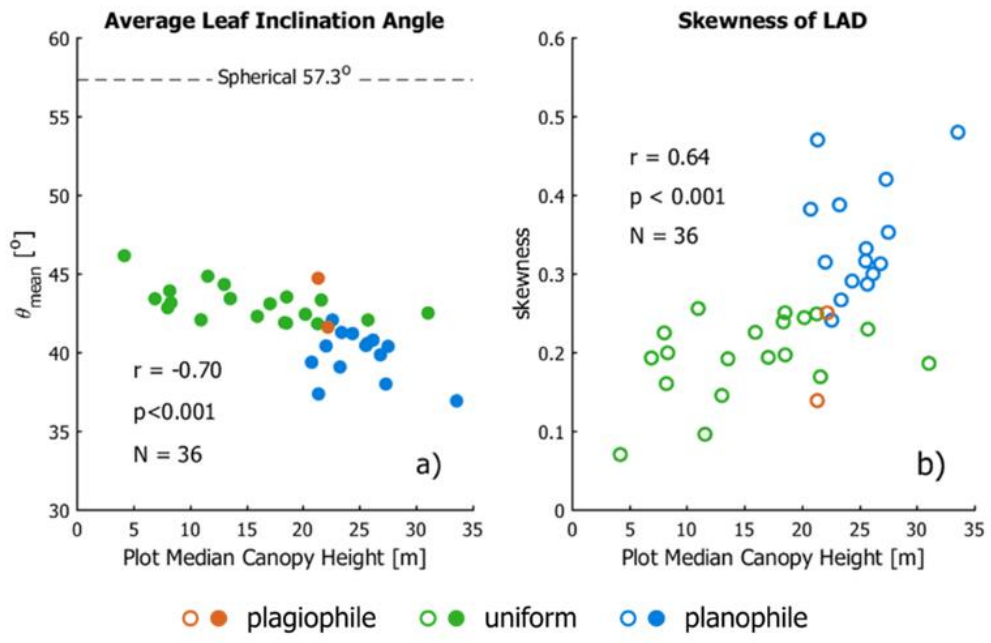

Figure 3.9: The correlation between plot median canopy height and plot a) average leaf inclination angle and b) skewness of leaf angle distribution (LAD) across all 36 European beech plots

The LAD variation at different canopy layers in all 36 plots is shown in Fig 3.10. From paired sample $t$ test, for all plots, the LAD in the top layer has the highest $\theta_{\text {mean }}(p<0.001)$, the highest $\theta_{\text {mode }}(p<0.001)$ and the lowest skewness $(p<0.001)$. There is no statistical significant difference in $\theta_{\text {mean }}(p=0.586), \theta_{\text {mode }}(p=0.704)$ and skewness $(p=0.69)$ of the middle layer and bottom layer. This indicates that beech leaves in the top layer grow more vertically. From Fig 3.10, in young and medium 
plots when $\mathrm{CHM}_{\text {median }}$ is below $20 \mathrm{~m}$, the top layer has a spherical or plagiophile $\operatorname{LAD}\left(\theta_{\text {mean }} \in\left(47^{\circ}, 55^{\circ}\right)\right.$, skewness $\left.\in(-0.4,0)\right)$. In contrast, the middle and bottom layer have an uniform $\operatorname{LAD}\left(\theta_{\text {mean }} \in\left(41^{\circ}, 47^{\circ}\right)\right.$, skewness $\in(0,0.4))$. In most mature plots, when $\mathrm{CHM}_{\text {median }}$ is above $20 \mathrm{~m}$, the top layer becomes uniform or plagiophile $\operatorname{LAD}\left(\theta_{\text {mean }} \in\left(43^{\circ}, 51^{\circ}\right)\right.$, skewness $\in(-0.2,0.1))$. The middle and bottom layer have a planophile $\operatorname{LAD}\left(\theta_{\text {mean }} \in\left(33^{\circ}, 45^{\circ}\right)\right.$, skewness $\left.\in(0.1,0.8)\right)$.

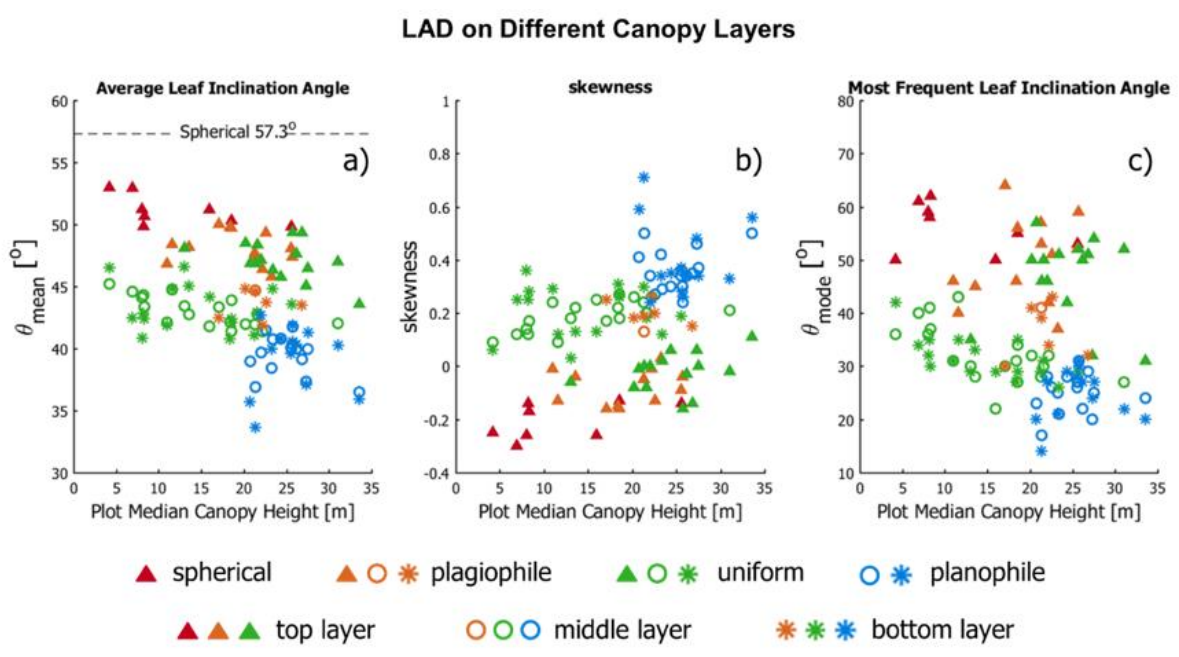

Figure 3.10: LAD variation at different canopy layers and across different stands, a) average leaf inclination angle; b) most frequent leaf inclination angle; c) skewness of LAD

Additional statistics can be seen in Table 3.5. Out of all 108 layers from the 36 plots, there is a large variation of $\theta_{\text {mean }}\left(33.64^{\circ}-52.97^{\circ}\right)$, while the variation of $\theta_{\text {mode }}$ is even higher $\left(14^{\circ}-64^{\circ}\right) .20$ of the 36 plots have an inner plot vertical $\theta_{\text {mean }}$ difference of more than $15 \%$ (maximum difference $=28.18 \%$, from $33.64^{\circ}$ to $46.84^{\circ}$ ). 23 plots have an inner plot vertical $\theta_{\text {mode }}$ difference of more than $40 \%$ (maximum difference $=$ $69.57 \%$, from $14^{\circ}$ to $46^{\circ}$ ). This demonstrates inner-plot vertical variation of LAD is even more severe than inter-plot variation of LAD. 


\section{Results}

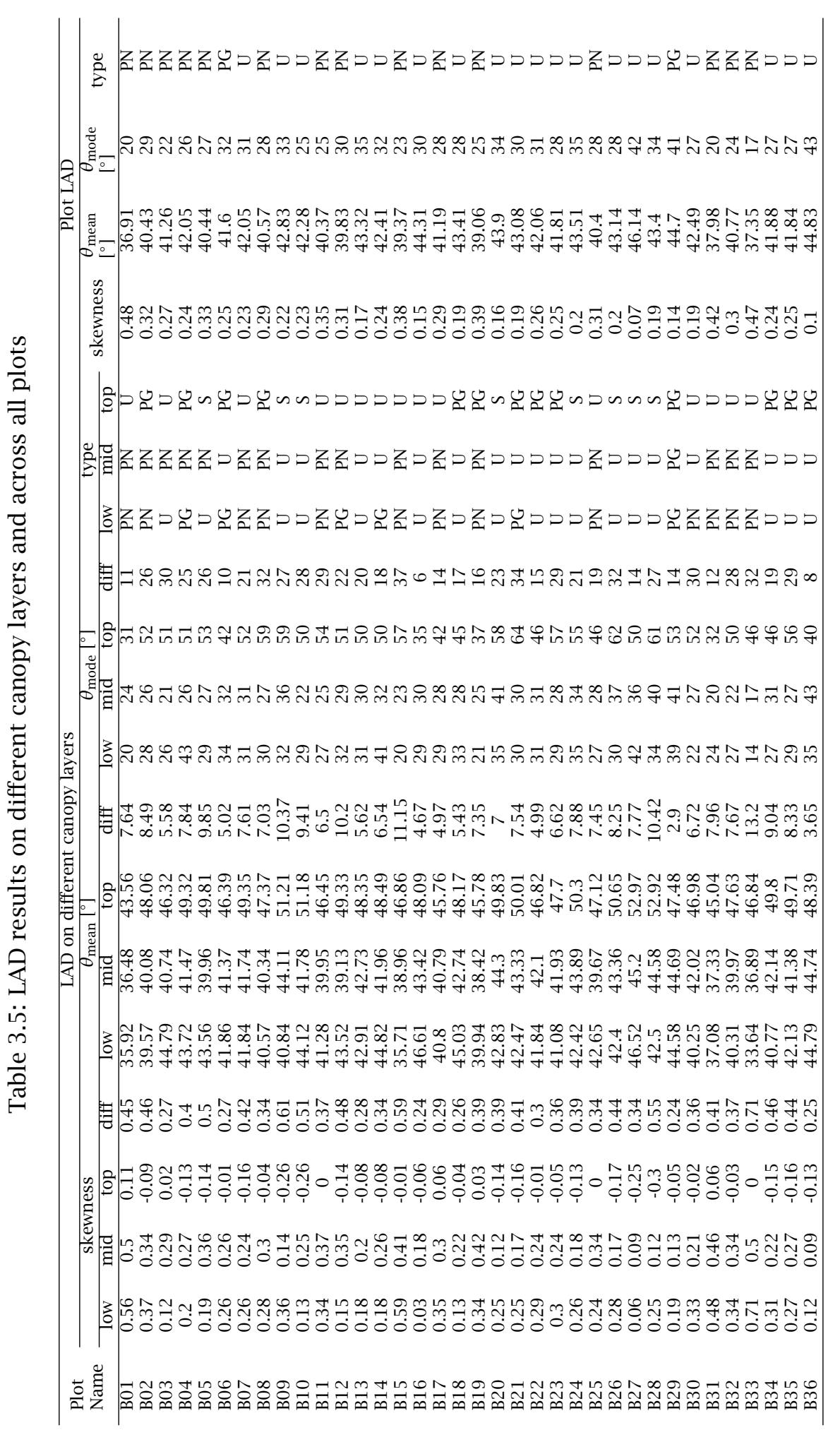




\section{Discussion}

\subsection{Variation of leaf angle distribution in European beech forests}

In this research, moderate LAD variation was found across European beech plots in different succession status, and they are all quite different from the spherical distribution. This led us to question the widespread simplification of LAD as the spherical distribution (Richardson et al., 2009; Tang et al., 2012) in previous research. In mature stands, we found planophile LAD, which is consistent with previous research where the average leaf inclination angle of beech forests is reported to be around $21.7^{\circ}$ (Wagner and Hagemeier, 2006), 31 (Chianucci et al., 2015) and $18.08^{\circ}$ (Chianucci et al., 2018). It is also consistent with the planophile LAD suggestion for temperate broadleaf forests (Pisek et al., 2013). However, in young and medium stands, LAD is most similar to the uniform rather than the planophile distribution.

An important new discovery from this research is that there is a strong negative correlation $(r=-0.70, p<0.001)$ between the median canopy height of a plot and the average leaf inclination angle $\theta_{\text {mean }}$ of the plot, for natural European beech forests. From young to mature stands, LAD changed from symmetric uniform or plagiophile distribution to a skewed planophile distribution. This implies that in situations where canopy height or stand age data is available, it is possible to estimate plotspecific LAD. This offers the potential to upscale this study and map LAD at regional scale using airborne or satellite LiDAR data.

There is even larger variation of $\mathrm{LAD}$ on different canopy layers ( $\theta_{\text {mean }} \in$ [33.64 ${ }^{\circ}$ to $\left.52.97^{\circ}\right], \theta_{\text {mode }} \in\left[14^{\circ}\right.$ to $\left.\left.64^{\circ}\right]\right)$ quantified by TLS. A general trend is that leaves grow more vertically in the top canopy layer, and grow more horizontally in the middle and bottom layers. This is consistent with previous studies, where leaves were found more horizontal in understory beech saplings (Planchais and Pontailler, 1999; Balandier et al., 2007; Chianucci et al., 2014) or shaded beech saplings (Delagrange et al., 2006). Based on the results in 4.3 and 4.4, we recommend when in-situ LAD data are not available, the choice of predefined functions for LAD approximation could follow instructions in Table 3.6. 
Table 3.6: Suggested choice of predefined functions for LAD approximation in European Beech stands

\begin{tabular}{lcccc}
\hline & \multirow{2}{*}{ previous } & \multicolumn{2}{c}{ from this research } \\
\cline { 3 - 5 } & & & young or medium stands & mature stands \\
\hline \multirow{3}{*}{ multilayer } & top & $\backslash$ & spherical & uniform \\
& middle & $\backslash$ & uniform & planophile \\
plot averaged & bottom & $\backslash$ & uniform & planophile \\
& & spherical & uniform & planophile \\
\hline
\end{tabular}

There may be many reasons for such variation of LAD in European beech forests. LAD can be viewed as a morphological or structural trait of plants. Plant traits can reflect the outcome of evolutionary and community assembly processes responding to abiotic and biotic environmental constraints (Valladares et al., 2007; Kattge et al., 2011). The vertical variation of LAD in European beech plots can be interpreted as a result of plant adaptation to different light availability at different canopy layers. On the one hand, leaves in the top layer have a higher chance of direct sun, so steeper leaf angles can help reduce exposure to excess radiation and consequent water stress during the middle of the day (Falster and Westoby, 2003), as well as allowing more light to reach the lower canopy. On the other hand, leaves in the middle and bottom layer are more likely to be shaded leaves, with a flatter inclination enhancing light interception under low light levels (Niinemets, 2010). These mid to low canopy position leaves are also less susceptible to high evapotranspiration due to canopy shading (Ryu et al., 2011). The more horizontal inclination may also be a strategy to eliminate competition from other species (Niinemets, 2010). A similar trend of more planophile LAD in lower canopy was also found in an oak forest in the UK (Kull et al., 1999), a mixed deciduous forest in the US (Hutchison et al., 1986) and a mature tropical moist forest in Republic of Panama (Wirth et al., 2001), where researchers used only one stand and estimated LAD manually. The negative correlation of LAD with the median canopy height of the plot, may be due to the increasing percentage of leaves under low light conditions inside the canopy relative to the crown periphery. This led to an increasing percentage of leaves with low leaf inclination angles, thus leading to a decrease in $\theta_{\text {mean }}$. 


\subsection{Factors affecting LAD retrieval from TLS}

Compared to the manual measurement and levelled digital canopy photography method, TLS has the advantage of rapid data acquisition of large area, dense sampling, efficient automatic processing and less human intervention (e.g., no need to identify each leaf visually), let alone its advantage to acquire millimeter level precise geometrical information. Although this study was conducted in European beech forests, the TLS method can be transferred to many other broadleaf species, most suitable for flat leaves. It may also be used to large non-flat leaf like corn, where individual leaf can be regarded consisting of several flat patches. In the following, we will discuss some aspects, which should be considered when using TLS to quantify LAD.

\subsubsection{TLS data collection and preprocessing}

High quality point cloud data are prerequisite for LAD retrieval from TLS. A small beam divergence, small angular step, and close range is necessary for plants with small leaves. Otherwise the TLS footprint may be larger than individual leaf, making leaf surface reconstruction impossible. The optimal settings can be calculated from the parameters of the TLS sensor and plant leaf size. For more information, one can refer to a previous research (Wilkes et al., 2017). A multiple scan position design and a small angular step are suggested to increase point density. Accurate coregistration among multiple scans ensures utilization of all scan points. While a bad co-registration and misalignment may lead to many isolated points in the leaf surface reconstruction step or create spurious objects. In addition, raw height was used in this research instead of local height, since LAD estimation involves 3D structure and topology of neighboring points. Topographic normalization will create distortion of the point cloud (Liu et al., 2017a).

\subsubsection{Extraction of leaf points}

The classification of leaf versus woody points was very accurate in general. The main errors appear to be caused by fine scale twigs, which were wrongly classified as leaf points. This may result from high uncertainty 
in twig point reflectance due to the "partial hit". Because twigs are very narrow, there is a higher possibility for a laser beam to partially hit the twig and only a fraction of the laser pulse is returned (Eitel et al., 2010). In this condition, the "calibrated relative reflectance" measured by Riegl VZ-400 is not valid, since the partial illumination of a bright target can yield the same measurement as a more complete illumination of a darker target (Beland et al., 2014). As a result, the reflectance of twigs was lower than other woody points, causing incorrect classification as leaves.

\subsubsection{Point density effect}

A potential source of uncertainty in leaf angle distribution (LAD) is the point density effect. In TLS, the spherical scanning geometry leads to a higher point density of near-range objects than far-range objects (Jupp et al., 2009; Zhao et al., 2015). Point density was shown to influence the retrieval accuracy of canopy height, canopy cover, and biomass (Jakubowski et al., 2013; Wilkes et al., 2015b; Garcia et al., 2017). Therefore, the point density effect on leaf angle calculation was analyzed. We used the mean nearest neighbor points distance (MNNPD) (Wilkes et al., 2017) to quantify point density. Sparse point clouds have high MNNPD while dense point clouds have high MNNPD. In all 36 beech plots, with increasing height above ground, MNNPD increases from $0.24 \mathrm{~cm}$ to $2.86 \mathrm{~cm}$ shown in Fig 3.11 a). To mimic this point density change, the simulated TLS point cloud of the synthetic young beech tree was thinned to different levels. First, we randomly selected points from the raw TLS data at different percentages, from $5 \%$ to $20 \%$ at a step of $1 \%$, from $20 \%$ to $100 \%$ at a step of $5 \%$. In total 32 point clouds were generated. Second, the leaf angle calculation and accuracy evaluation was implemented for each point cloud. From the results (Fig $3.11 \mathrm{~b}$ ), leaf angle estimation became less accurate ( $R^{2}$ : from 0.88 to 0.52 , RMSE: from $8.37^{\circ}$ to $12.39^{\circ}$ ) with a decreasing point density (or increasing MNNPD). It can be concluded that at a height of $0-20 \mathrm{~m}$ above ground, the leaf angle estimation is very accurate (MNNPD $<1.4 \mathrm{~cm}, R^{2}>0.75$, RMSE $<9.5^{\circ}$ ). From $20-30 \mathrm{~m}$, leaf angle estimation is moderately accurate (MNNPD $<2.4 \mathrm{~cm}, R^{2}>0.6$, RMSE $<11.5^{\circ}$ ). Above $30 \mathrm{~m}$, leaf angle estimation is roughly accurate (MNNPD $<3 \mathrm{~cm}, R^{2}>0.5$, RMSE $<13^{\circ}$ ). In future studies, for more accurate leaf angle measurements of tree tops (above $30 \mathrm{~m}$ ), it is recommended to 
combine with TLS scans below canopy with an UAV LiDAR scan above canopy.
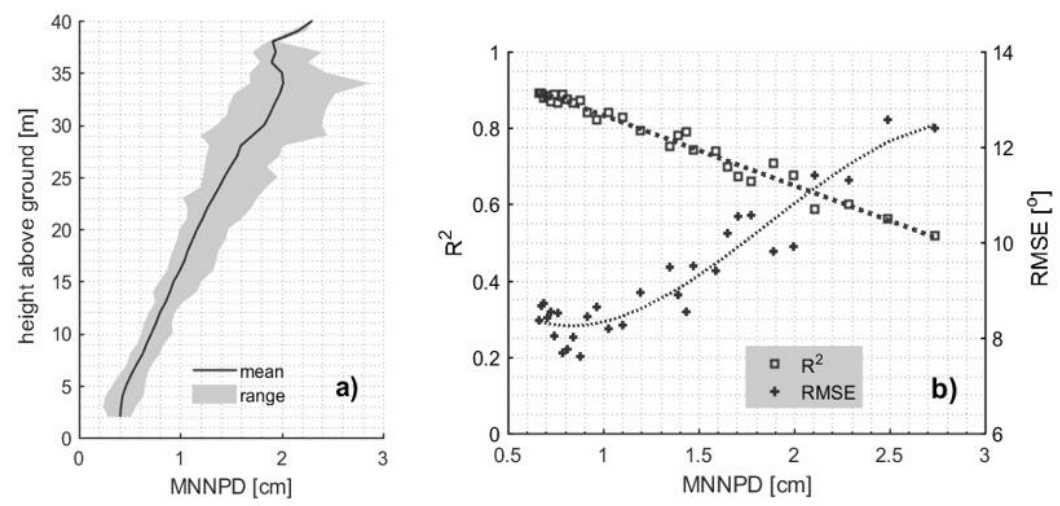

Figure 3.11: a) The decreasing point density with height above ground in all 36 beech plots (point density was quantified by mean nearest neighbor points distance (MNNPD); b) leaf angle estimation accuracy decreases with decreasing point density from the simulated point clouds

\subsubsection{Evaluating the accuracy of leaf angle distribution retrieval}

Evaluating the LAD accuracy is extremely difficult due to challenges in manually measuring true leaf angle. Especially in natural forests, it is virtually impossible to find nearby tall buildings or observation towers as used in previous studies (Raabe et al., 2015), to remotely observe and measure leaf angles. Although it is possible to use ladders or tree climbing to reach higher levels in a tree, the movement usually disturbs the canopy and changes leaf angles (Zheng and Moskal, 2012). Therefore, in this study, the accuracy of the proposed method was evaluated using a simulated dataset. The results demonstrated that the leaf angle calculation were very accurate in general. It should be highlighted that compared to terrestrial LiDAR data scanned from real forests, the simulation data have perfect registration, less noisy points, and no errors for woody material classification (since the material type is known from the beech tree model). Future studies may investigate the effect of these factors on LAD measurement. 


\subsection{Management implications}

The findings in this research may improve the accuracy in LAI mapping. Airborne and spaceborne LiDAR has been increasingly used to map LAI (Korhonen et al., 2011; Stark et al., 2015; Tang and Dubayah, 2017). In these studies, gap fraction was first estimated by laser pulse penetration. Then LAI could be estimated based on the gap fraction model,

$$
P_{\text {gap }}(\theta)=e^{-G(\theta) \lambda L / \cos (\theta)}
$$

where $\theta$ is the direction of incoming radiation, $P_{\text {gap }}(\theta)$ is the gap fraction in direction $\theta, L$ is the LAI, $\lambda(\theta)$ is the clumping index, $G(\theta)$ is the leaf projection function determined by LAD. However in this method, an accurate estimate of $G(\theta)$ is necessary for accurate LAI estimate. The spherical LAD distribution was preferred in most cases because the $G(\theta)$ can be approximated as 0.5 in any direction (Fig 3.12). But unfortunately airborne LiDAR usually operates at small scan angles in $0^{\circ}-30^{\circ}$ (Liu et al. 2018). At this range, there is large difference among $G(\theta)$ of different LAD (Fig 3.12). If stand specific LAD can be quantified, there is potential to increase LAI mapping accuracy.

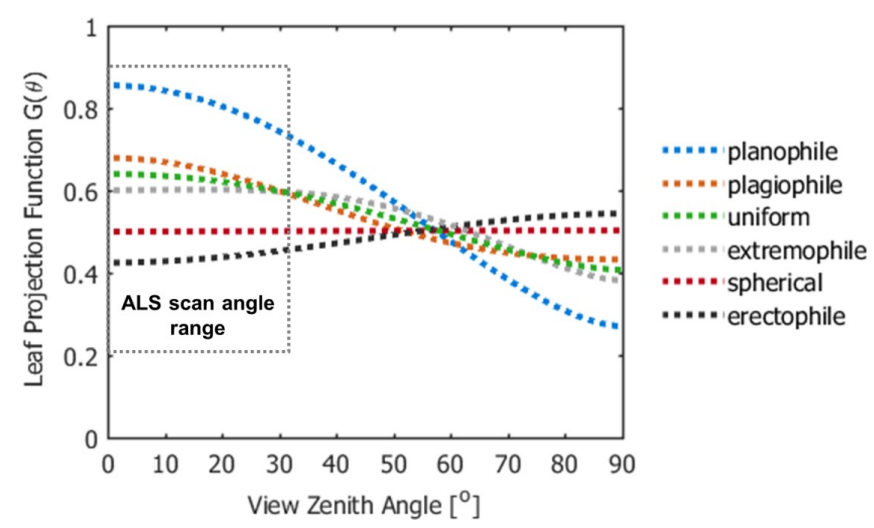

Figure 3.12: The value of leaf projection function $\mathrm{G}(\theta)$ under 6 predefined leaf angle distribution (LAD) assumptions and commonly used airborne LiDAR (ALS) scan angle range

In addition, the vertical LAD variation at different canopy layers, demonstrates the necessity for multi-layer radiative transfer modelling (Kuusk, 2001; Yang et al., 2017), even for the same species. The method used in 
this research, could also be utilized to study plant function, forest ecology and evolution. For example, leaf inclination may change due to water stress (Biskup et al., 2007), leaf expansion and senescence. Terrestrial LiDAR could be used to acquire measurements during the live cycle of leaves under varying microclimatic conditions. The vertical variation of LAD in European beech stands as found in this research, are consistent with the discovery that late-successional stands dominated by shade tolerant species often have a more horizontal leaf inclination angles (McMillen and McClendon, 1979; Pearcy et al., 2004; Niinemets, 2010). Further research could use terrestrial LiDAR to acquire measurements in different forest types, to explore the relationship between geography and plant structural traits.

\section{Conclusion}

In this study, the variation of leaf angle distribution (LAD) in European beech (Fagus sylvatica) forests was examined using terrestrial LiDAR. A total of 36 plots ranging from young, medium to mature successional status were studied. Leaf and woody materials were differentiated based on a combination of radiometric and geometric features. Leaf surface was reconstructed and leaf inclination angles were subsequently calculated. From the statistical results, we conclude:

1. Terrestrial LiDAR proves to be an effective tool to quantify LAD variation due to its capability to acquire massive data rapidly, differentiate leaf and woody materials, and provide precise 3D information.

2. There is moderate variation of LAD across beech plots at different successional status. Instead of a spherical LAD assumption, it is more valid to assume a uniform LAD for young and medium stands, a planophile LAD for mature stands.

3. There is large variation of LAD on different canopy layers. Beech leaves grow more vertically in the top layer, but more obliquely or horizontally in the middle and bottom layer.

4. A strong negative correlation exists between the plot average leaf angle and the plot median canopy height. This offers the potential 
to estimate plot specific LAD from canopy height data in European Beech forests.

5. Large variation of LAD should be accounted for better LAI mapping and canopy photosynthesis modelling. 



\section{Scan angle impact on gap fraction estimation from airborne LiDAR *}

"This chapter is based on: Liu, J., Skidmore, A., Jones, S., Wang, T., Heurich, M., Zhu, X., Shi, Y., 2018. Large off-nadir scan angle of airborne LiDAR can severely affect the estimates of forest structure metrics. ISPRS Journal of Photogrammetry and Remote Sensing, 136, 13-25. 


\begin{abstract}
Gap fraction $\left(P_{\text {gap }}\right)$ and vertical gap fraction profile (vertical $P_{\text {gap }}$ profile) are important forest structure metrics. Accurate estimation of $P_{\text {gap }}$ and vertical $P_{\text {gap }}$ profile is therefore critical for many ecological applications, including leaf area index (LAI) mapping, vertical LAI profile estimation and wildlife habitat modelling. Although many studies estimated $P_{g a p}$ and vertical $P_{\text {gap }}$ profile from airborne LiDAR data, the scan angle was often overlooked and a nadir view assumed. However, the scan angle can be off-nadir and highly variable in the same flight strip or across different strips.
\end{abstract}

In this research, the impact of off-nadir scan angle on $P_{\text {gap }}$ and vertical $P_{\text {gap }}$ profile was evaluated, for several forest types. Airborne LiDAR data from nadir $\left(0^{\circ}-7^{\circ}\right)$, small off-nadir $\left(7^{\circ}-23^{\circ}\right)$, and large off-nadir $\left(23^{\circ}-38^{\circ}\right)$ directions were used to calculate both $P_{\text {gap }}$ and vertical $P_{\text {gap }}$ profile. Digital hemispherical photographs (DHP) acquired during fieldwork were used as references for validation. Our results show that angular $P_{g a p}$ from airborne LiDAR correlates well with angular $P_{\text {gap }}$ from DHP $\left(R^{2}\right.$ $=0.74,0.87$, and 0.67 for nadir, small off-nadir and large off-nadir direction). But underestimation of $P_{\text {gap }}$ from LiDAR amplifies at large offnadir scan angle. By comparing $P_{\text {gap }}$ and vertical $P_{g a p}$ profiles retrieved from different directions, it is shown that scan angle impact on $P_{\text {gap }}$ and vertical $P_{\text {gap }}$ profile differs among different forest types. The difference is likely to be caused by different leaf angle distribution and canopy architecture in these forest types. Statistical results demonstrate that the scan angle impact is more severe for plots with discontinuous or sparse canopies. These include coniferous plots, and deciduous or mixed plots with between-crown gaps. In these discontinuous plots, $P_{\text {gap }}$ and vertical $P_{\text {gap }}$ profiles are maximum when observed from nadir direction, and then rapidly decrease with increasing scan angle.

The results of this research have many important practical implications. First, it is suggested that large off-nadir scan angle of airborne LiDAR should be avoided to ensure a more accurate $P_{\text {gap }}$ and LAI estimation. Second, the angular dependence of vertical $P_{\text {gap }}$ profiles observed from airborne LiDAR should be accounted for, in order to improve the retrieval of vertical LAI profiles, and other quantitative canopy structural 
metrics. This is especially necessary when using multi-temporal datasets in discontinuous forest types. Third, the anisotropy of $P_{\text {gap }}$ and vertical $P_{\text {gap }}$ profile observed by airborne LiDAR, can potentially help to resolve the anisotropic behavior of canopy reflectance, and refine the inversion of biophysical and biochemical properties from passive multispectral or hyperspectral remote sensing data. 


\section{Introduction}

In forest ecosystems, within-crown and between-crown gaps are important functional and structural properties in forest canopies. Their ecological importance has long been recognized because of their role in controlling the transmission and interception of light (Ross, 1981), water vapor (Anderson et al., 1969), and snow (Hedstrom and Pomeroy, 1998), thus affecting the microclimate and soil moisture of the forest stand (Prescott, 2002). Gaps also contribute to the regeneration of trees (Gray and Spies, 1996), drive successional dynamics (Schnitzer and Carson, 2001), and increase overall species diversity.

Gap fraction $\left(P_{\text {gap }}\right)$ is a quantitative metric of forest gaps, defined as the probability of a ray of light passing directly through the canopy to a reference level (generally the ground), without being intercepted by a leaf, branch, or stem (Nilson, 1971). From a remote sensing perspective, $P_{\text {gap }}$ is equivalent to the probability that ground surface is directly visible to airborne and spaceborne platforms (Armston et al., 2013; Chen et al., 2014). Vertical gap fraction profile (vertical $P_{g a p}$ profile) is a function of the $P_{\text {gap }}$ with height. It describes the gap fraction value at each height above ground in the canopy. Many other canopy structural properties, including fractional cover, leaf area index (LAI), vertical LAI profile, above-ground biomass can be modelled using different expressions and combinations of canopy height and $P_{\text {gap }}$ (Armston et al., 2013).

The development of LiDAR technology has advanced the mapping of $P_{\text {gap }}$. Early research used airborne LiDAR penetration variables to estimate $P_{\text {gap }}$ and then LAI was estimated based on the Beer-Lambert law (Riaño et al., 2004; Solberg et al., 2009). A growing body of research also exploited the ranging advantage of LiDAR, to estimate the vertical $P_{\text {gap }}$ profile and vertical LAI profile using terrestrial (Lovell et al., 2003; Jupp et al., 2009), airborne (Coops et al., 2007; Hilker et al., 2010) or spaceborne (Tang et al., 2014b) LiDAR data. The vertical $P_{\text {gap }}$ profile and the vertical LAI profile have been further utilized in a broad range of ecological applications, including modelling avian species diversity (Goetz et al., 2007), modelling carbon dynamics (Kotchenova et al., 2004; Stark et al., 2012), mapping forest succession stages (Falkowski et al., 2009), modelling fire susceptibility (de Almeida et al., 2016), quantifying canopy 
strata (Wilkes et al., 2015a), quantifying forest understory growth (Tang and Dubayah, 2017), and monitoring vegetation phenology (Calders et al., 2015; Griebel et al., 2015).

As such an important prerequisite metric, accurate measurement of $P_{g a p}$ is critical to subsequent applications. In recent years, researchers have used airborne LiDAR data with a large scan angle range (up to $\pm 45^{\circ}$ ) in forests (Morsdorf et al., 2010; Cao et al., 2016; Marino et al., 2016). With this configuration, there is high variation in scan angles, even in the same flight. For forest managers or researchers using multi temporal LiDAR flights, it is more difficult to maintain exactly the same settings and scan angle. Previous research has shown that the precision of canopy height estimates decreased with increasing off-nadir scan angle (Lovell et al., 2005; Pang et al., 2011). Also, lower height percentiles were affected more than upper height percentiles, due to obscuration and longer path of laser pulse penetrating into canopy (Holmgren et al., 2003). However, in $P_{\text {gap }}$ and vertical $P_{\text {gap }}$ profile mapping, the scan angle was often overlooked and a nadir scanning geometry was assumed (Hilker et al., 2010; Stark et al., 2012; Tang et al., 2012). The potential impacts of off-nadir scan angle on $P_{\text {gap }}$ and vertical $P_{\text {gap }}$ profile estimation have rarely been studied.

In a previous study, $P_{\text {gap }}$ was found to decrease with increasing zenith angle (Liu et al., 2008), in four plots dominated by Engelmann spruce (Picea engelmannii), subalpine fir (Abies lasiocarpa) and lodgepole pine (Pinus contorta). But this result was observed from a geometric optical radiative transfer modelling instead of direct measurement from airborne LiDAR (Liu et al., 2008). In other research, the accuracy of estimated $P_{\text {gap }}$ from airborne LiDAR was found to be influenced by the range of scan angles. Using a small range of scan angles $\left(0^{\circ}-15^{\circ}\right)$, the $P_{\text {gap }}$ from ground measurement had a good linear 1:1 correlation with the $P_{\text {gap }}$ estimated from LiDAR, with low root-mean-square error (RMSE) and bias. But at a large range of scan angles $\left(0^{\circ}-75^{\circ}\right)$, the $P_{\text {gap }}$ from ground measurement had a strong but nonlinear dependency with the $P_{\text {gap }}$ from LiDAR (Korhonen et al., 2011). However, this comparison may not be appropriate because the $0^{\circ}-75^{\circ}$ angle range is very large for direct comparison between ground-measured $P_{\text {gap }}$ and LiDAR-derived $P_{\text {gap }}$. Unlike ground-based techniques such as digital hemispherical 
photography (DHP) or LAI-2200 which can sample the entire hemisphere, airborne LiDAR measurements usually sample the plot at only limited scan angle range. The high cost of acquiring multi-angle airborne LiDAR data covering the same area is probably one of the biggest challenges impeding research on the scan angle impact. Typically, a limited angular range can be acquired for a forest plot in each airborne LiDAR flight strip. In order to acquire multi-angle data, highly overlapping LiDAR flight lines are required. Using multi-angle airborne LiDAR data, Zheng et al. (2017) observed anisotropy of $P_{\text {gap }}$ in a broadleaf plot. They found that the $P_{\text {gap }}$ was different both at different zenith angles and at different azimuth angles. The author also suggested that the scan angle effect should be corrected for a more accurate effective LAI estimation (Zheng et al., 2017). In terms of scan angle impact on vertical $P_{\text {gap }}$ profile, there has been some inspiring observation in one study, where significant differences among vertical $P_{\text {gap }}$ profiles derived from different scan angles have been found (Jupp et al., 2009). From $0^{\circ}$ nadir to $10^{\circ}$ off-nadir direction, there is very high variance in derived vertical $P_{\text {gap }}$ profiles. The author attributed these differences to the clumping effect and large gaps near the nadir direction (Jupp et al., 2009). However, this research was conducted using a terrestrial LiDAR, of which the scanning pattern is quite different from airborne LiDAR. Also the experiment was conducted in only one coniferous (Pinus ponderosa) stand, whether other forest types will also be impacted by scan angle or not remains unknown.

In this chapter, airborne LiDAR data acquired at different scan angles was used to assess the scan angle impact on $P_{\text {gap }}$ and vertical $P_{\text {gap }}$ profile, in several forest types. Specifically, the capability and accuracy of angular $P_{\text {gap }}$ derived from airborne LiDAR was first examined. Then, the scan angle impact was explored through analyzing the difference between $P_{\text {gap }}$ or vertical $P_{\text {gap }}$ profile results derived from LiDAR data of different scan angles. Possible reasons and implications of the anisotropy characteristics of $P_{g a p}$ and vertical $P_{g a p}$ profile were also discussed. 


\section{Materials and method}

\subsection{Study Area}

This study was conducted in the Bavarian Forest National Park in the southern-eastern part of Germany (N 49 $3^{\prime} 19^{\prime \prime}$, E $\left.13^{\circ} 12^{\prime} 9^{\prime \prime}\right)$. The park has a total area of 24218 hectares, with elevation ranging from about 600 to $1450 \mathrm{~m}$ above sea level (Heurich et al., 2010). The forest is dominated by Norway spruce (Picea abies) (67\%) and European beech (Fagus sylvatica) (24.5\%). Above $1100 \mathrm{~m}$ many lying and standing deadwood areas can be found on the mountain summit. Between $600 \mathrm{~m}$ and $1100 \mathrm{~m}$, white fir (Abies alba) and sycamore maple (Acer pseudoplatanus) occur. Wet depressions in the valleys harbor highly mixed forest with Norway spruce, mountain ash (Sorbus aucuparia) and birches (Betula spp.) (Cailleret et al., 2014). Due to natural disturbance and the non-intervention management policy, there is high structural complexity in the study area. Typical pure deciduous European beech stands, pure coniferous Norway spruce stands and mixed stands can be seen in Fig 4.1.
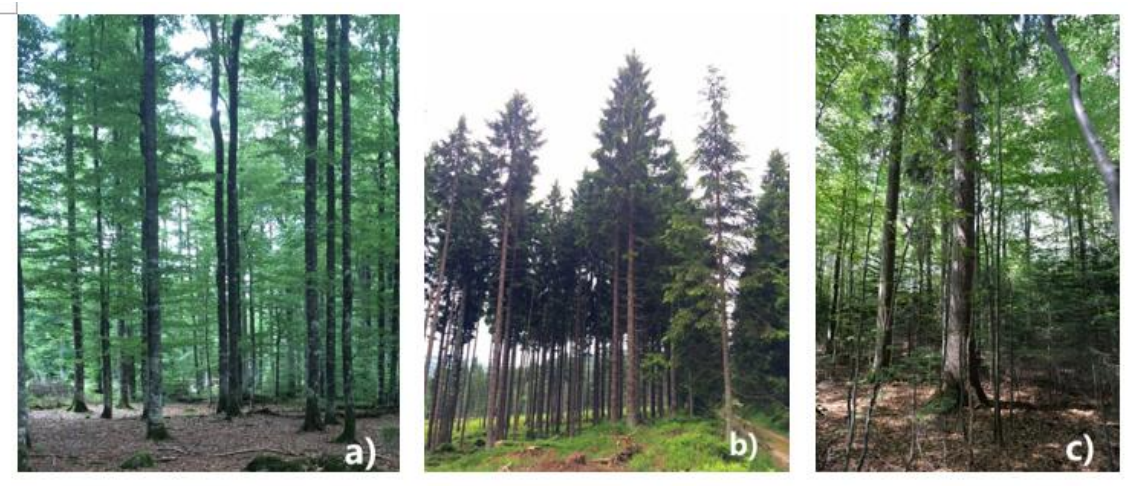

Figure 4.1: Examples of a) European beech, b) Norway spruce, and c) mixed stands in the study area

\subsection{Airborne LiDAR data}

The airborne LiDAR data was collected by Milan Flug GmbH in August 2016, covering four transects (Fig 4.2) in the park. These four transects spanned from the valleys to mountain tops, encompassing different 
types of forest structures in the national park (Bässler et al., 2008). The sensor onboard was a Riegl LMS-Q680i laser scanner, operating at a wavelength of $1550 \mathrm{~nm}$, with a beam divergence of $0.5 \mathrm{mrad}$. The flying altitude was approximately $300 \mathrm{~m}$ above ground, with a pulse repetition frequency of $400 \mathrm{KHz}$. This led to a nominal footprint size of about $0.15 \mathrm{~m}$, and the maximum scan angle was as high as $38^{\circ}$. The average point density for single LiDAR flight line was 70 points per $\mathrm{m}^{2}$. Multiple overlapped flight lines were used with 30\%-50\% side overlaps. As a result, the point density in overlapped areas could be double or triple the point density in single flight line covered areas. These overlaps created more chance for the same areas being viewed from multiple scan angles. Point cloud data generated from Gaussian decomposition (Wagner et al., 2006) was delivered by Milan Flug. Up to eight returns can be recorded for each pulse. The discrete multiple return point cloud data composed of planimetric coordinates ( $\mathrm{x}$ and $\mathrm{y}$ ), ellipsoidal heights (z), return intensity, return number, class label, scan angle, echo width, number of total returns for a laser shot, and the GPS timestamp of the return.

\subsection{Forest plot selection}

In order to obtain LiDAR data from multiple scan angles, overlapped (between flying tracks) areas are the primary selection areas. 30 established forest plots (Table 4.1) were chosen from the Bioklim project (Bässler et al., 2008) and visited during the fieldwork from 21 June to 2 July 2016. The $P_{\text {gap }}$ was measured using ground-based DHP in these 30 plots for evaluating the accuracy of the $P_{\text {gap }}$ derived from LiDAR data. To increase the sample size for analyzing scan angle impact and statistical analysis, another 77 plots were also selected from the overlapped areas. As a result, a total of 107 circular plots (Fig 4.2), each with a radius of $20 \mathrm{~m}$, were clipped from the airborne LiDAR dataset. There were 36 coniferous, 31 deciduous and 40 mixed plots. Only pulses having all returns located inside the $20 \mathrm{~m}$ radius cylinder were kept. These plots cover a wide range of forest structures. The plots were categorized into three forest types including deciduous, coniferous, and mixed plots, using ancillary land cover classification data and digital aerial photograph $(0.25 \mathrm{~m}$ spatial resolution) acquired on 5 June 2015. 


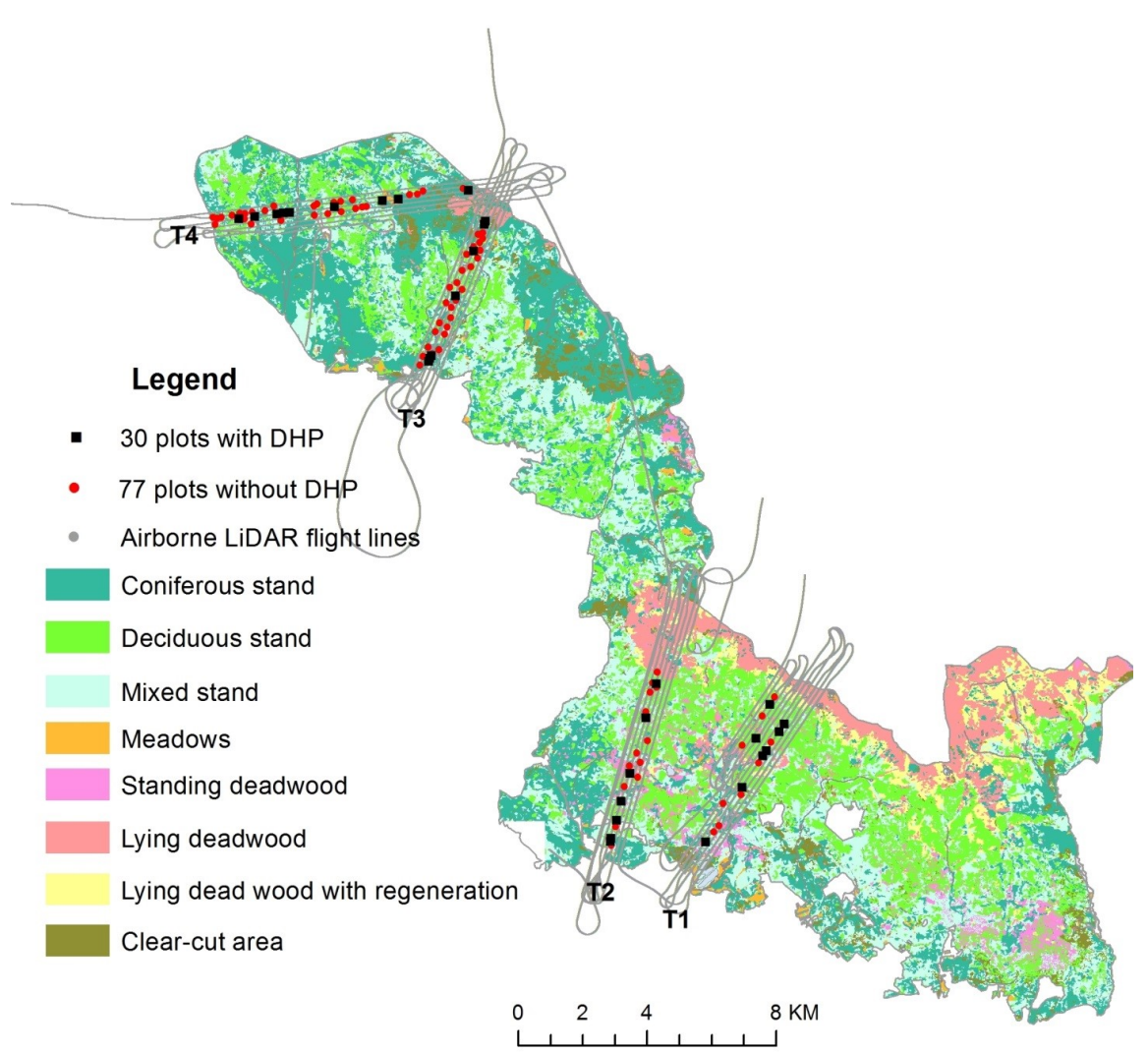

Figure 4.2: Airborne LiDAR flight lines and selected forest plots on the four transects (T1, T2, T3, T4) in the study area of Bavarian Forest National Park (DHP: digital hemispherical photograph)

All subsequent analysis in this research was conducted in these plots. The plot distribution and the airborne LiDAR flight lines can be seen in Fig 4.2. 
Table 4.1: Basic information of the 30 Bioklim plots with DHP measurements

\begin{tabular}{lccccccc}
\hline Plot & Forest Type & Long $\left[^{\circ}\right]^{\circ}$ & Lat $\left[{ }^{\circ}\right]$ & Ele $[\mathrm{m}]$ & $H_{\max }[\mathrm{m}]$ & $H_{\text {mean }}[\mathrm{m}]$ & Transect \\
\hline T4-25 & mix & 13.23 & 49.10 & 710 & 45.68 & 20.04 & 4 \\
T4-21 & mix & 13.23 & 49.10 & 734 & 52.6 & 23.86 & 4 \\
T4-14 & mix & 13.22 & 49.10 & 690 & 37.67 & 19 & 4 \\
T4-09 & mix & 13.21 & 49.10 & 741 & 47.12 & 21.95 & 4 \\
T3-49 & mix & 13.32 & 49.10 & 1181 & 22.8 & 6.51 & 3 \\
T3-40 & mix & 13.31 & 49.09 & 1106 & 34.59 & 16.88 & 3 \\
T3-25 & mix & 13.30 & 49.07 & 872 & 44.92 & 24.97 & 3 \\
T2-41 & mix & 13.38 & 48.96 & 1001 & 34.8 & 16.35 & 2 \\
T2-23 & mix & 13.37 & 48.94 & 842 & 26.2 & 3.81 & 2 \\
T2-08 & mix & 13.37 & 48.93 & 849 & 38.48 & 24.24 & 2 \\
T2-01 & mix & 13.36 & 48.92 & 785 & 41.26 & 25.36 & 2 \\
T1-52 & mix & 13.44 & 48.95 & 965 & 39.79 & 8.96 & 1 \\
T4-54 & deciduous & 13.27 & 49.10 & 1122 & 27.49 & 19.28 & 4 \\
T4-39 & deciduous & 13.25 & 49.10 & 788 & 43.95 & 26.74 & 4 \\
T4-23 & deciduous & 13.23 & 49.10 & 717 & 39.93 & 17.31 & 4 \\
T2-52 & deciduous & 13.38 & 48.97 & 1152 & 27.42 & 16.41 & 2 \\
T2-14 & deciduous & 13.37 & 48.93 & 831 & 39.96 & 20.68 & 2 \\
T2-02 & deciduous & 13.36 & 48.92 & 803 & 38.34 & 28.09 & 2 \\
T1-49 & deciduous & 13.44 & 48.95 & 914 & 30.59 & 12.77 & 1 \\
T1-08 & deciduous & 13.40 & 48.92 & 802 & 36.32 & 12.19 & 1 \\
B2 & deciduous & 13.43 & 48.96 & 997 & 32.52 & 22.77 & 1 \\
B1 & deciduous & 13.43 & 48.95 & 849 & 35.62 & 21.13 & 1 \\
T4-81 & coniferous & 13.31 & 49.10 & 1316 & 11.13 & 1.51 & 4 \\
T4-59 & coniferous & 13.28 & 49.10 & 1162 & 37.3 & 17.03 & 4 \\
T3-50 & coniferous & 13.32 & 49.05 & 1193 & 19.05 & 2.02 & 3 \\
T3-05 & coniferous & 13.29 & 49.06 & 703 & 14.84 & 5.01 & 3 \\
T3-04 & coniferous & 13.29 & 49.06 & 694 & 28.63 & 17.08 & 3 \\
T3-03 & coniferous & 13.29 & 49.06 & 684 & 31.71 & 16.58 & 3 \\
T1-42 & coniferous & 13.43 & 48.95 & 829 & 35.25 & 20.39 & 1 \\
T1-40 & coniferous & 13.43 & 48.95 & 815 & 16.53 & 3.56 & 1 \\
\hline
\end{tabular}

Long: longitude; Lat: latitude

Ele: elevation; $H_{\max }$ : maximum canopy height; $H_{\text {mean }}$ : average canopy height

\subsection{Digital hemispherical photograph}

From 21 June to 2 July 2016, digital hemispherical photographs (DHP) were acquired in the above-mentioned 30 plots (Fig 4.2). Previous research showed that $P_{\text {gap }}$ results from DHP may have high variance due to instrument calibration (Lang et al., 2010), sky illumination conditions (Pueschel et al., 2012), camera exposure (Zhang et al., 2005), and image classification method (Woodgate et al., 2015). To reduce uncertainty, DHP was carefully setup during this research as follows. In each plot, 20-30 upward-pointing DHPs were collected, first in the plot center, and then $10 \mathrm{~m}$ from the center in each diagonal direction. At each position, 
a digital camera (Canon EOS 5D) equipped with a fish-eye lens (Sigma 8mm F3.5 EX DF) was first set-up and leveled on a tripod between 1 to $1.3 \mathrm{~m}$ above ground. Photos were taken in diffuse light conditions at dusk or dawn, or overcast diffuse conditions during the day, using manual exposure. Each image had a high resolution of 5600 by 3898 pixels. In order to minimize subjective thresholding, the two-corner classification method (Macfarlane, 2011) was applied on the blue channel of all obtained images to classify sky and canopy pixels. Then, the binary classified images are imported into the CanEye (Weiss and Baret, 2010) software to calculate $P_{\text {gap }}$. Each classified image was broken into a series of annuli from $0^{\circ}-60^{\circ}$, with each ring of $2.5^{\circ}$ width. Angular $P_{\text {gap }}$ could then be derived for each annulus. The remaining $60^{\circ}-90^{\circ}$ annuli were excluded because of high portion of mixed pixels.

\section{5 $P_{\text {gap }}$ and vertical $P_{\text {gap }}$ profile}

The premise of using LiDAR to calculate $P_{\text {gap }}$ is that the interaction between forest canopy and laser pulses can be considered analogous to the interaction of forest canopy with direct beam solar radiation (Hopkinson and Chasmer, 2007). When a laser pulse comes into contact with an object in its path, part of the laser energy is reflected back to the laser sensor, and triggers the signal recording once the energy exceeds a threshold. In full waveform LiDAR, the entire return signal is recorded. The $P_{\text {gap }}$ can then be computed through the ratio between canopy backscattered energy and total backscattered energy, after correcting for the backscattering coefficient difference between ground and vegetation (Lefsky et al., 2002; Tang et al., 2012; Armston et al., 2013). However, for discrete return LiDAR, only the intensity of each individual return is recorded. Due to various reasons (such as proprietary waveform decomposition algorithms and lack of radiometric calibration), intensity can be uninformative and uncertain (Höfle and Pfeifer, 2007; Hancock et al., 2015). In these cases, $P_{\text {gap }}$ is usually approximated by various laser penetration rate variables including,

$$
P_{\text {gap }}=\frac{N_{\text {last-ground }}}{N_{\text {total }}}
$$




$$
P_{\text {gap }}=1-\frac{N_{\text {first-vege }}}{N_{\text {total }}}
$$

where $N_{\text {total }}$ is the total number of all received/recorded pulses, $N_{\text {last-ground }}$ is the number of pulses having the last return down to ground (Solberg et al., 2009). $N_{\text {first-vege }}$ is the number of pulses having the first return hit by vegetation (Lovell et al., 2003; Morsdorf et al., 2006). Since the airborne laser footprint size is usually larger than individual leaf size, equation 4.1 tends to overestimate $P_{\text {gap }}$ due to failure to account for within-crown vegetation, while equation 4.2 causes underestimation of the $P_{\text {gap }}$ due to failure to account for within-crown gaps. Therefore, in this research, the $P_{\text {gap }}$ is calculated using a "weighted all return" method which considers all returns in each pulse,

$$
P_{\text {gap }}=1-\frac{\sum 1 / N O R}{N_{\text {total }}}
$$

where $N_{\text {total }}$ is the total number of all received/recorded pulses, NOR is the number of returns for each pulse. 1/NOR is the weight assigned for each return in this given pulse. It serves as a robust estimate of return intensity. $\sum 1 / N O R$ is a weighted sum of all the returns hit by vegetation (excluding ground returns). This equation was proposed in previous research (Newnham et al., 2012; Armston et al., 2013) and proved to be capable of producing near unbiased estimates of $P_{\text {gap }}$ compared to a waveform method (Armston et al., 2013). When calculating vertical $P_{\text {gap }}$ profile as a function of height,

$$
P_{\text {gap }}(h)=1-\frac{\sum 1 / N O R(h)}{N_{\text {total }}}
$$

where $\sum 1 / \operatorname{NOR}(h)$ is a weighted sum of all the returns hit by vegetation and locating above the height of $h$ above ground. In this study, the vertical $P_{\text {gap }}$ profile was calculated using $0.2 \mathrm{~m}$ height bins (i.e., $0.2 \mathrm{~m}$ height intervals). Based on the gap fraction model, in the case of a turbid media with random spatial distribution of infinitely small leaves and random leaf azimuth distribution, the $P_{\text {gap }}$ in the direction $\theta$ can be related to LAI using,

$$
P_{\text {gap }}(\theta)=e^{-G(\theta) L / \cos (\theta))}
$$


where $G(\theta)$ is the leaf projection function and equals the projection of a unit area of plant constituents on a plane perpendicular to the direction, averaged over elements of all orientations (Ross, 1981), $L$ is the LAI. It has also been demonstrated that even when the turbid medium assumptions are not satisfied, $P_{\text {gap }}$ can still be expressed as an exponential function of LAI (Nilson, 1971; Weiss et al., 2004). In case of clumped canopies, a modified expression is,

$$
\begin{aligned}
& P_{\text {gap }}(\theta)=e^{-G(\theta) \lambda L / \cos (\theta)} \\
& k(\theta)=G(\theta) / \cos (\theta)
\end{aligned}
$$

where $\lambda$ is the clumping index (Weiss et al., 2004), $k(\theta)$ is the extinction coefficient (Campbell, 1986). Equation 4.6 serves as the basis for derivation of various other metrics such as LAI and clumping index.

\subsection{Quantification of scan angle impact and statistical analysis}

Although multiple scan angle LiDAR data can be acquired from overlapped flight lines, it was impossible to achieve the LiDAR data at a series of scan angles with small angular step. If a partition scheme like $0^{\circ}, 1^{\circ}$, $2^{\circ}, 3^{\circ}, \ldots, 38^{\circ}$ or $0^{\circ}, 2.5^{\circ}, 5^{\circ}, 7.5^{\circ}, \ldots, 37.5^{\circ}$ were used, it would be impossible for each sub point cloud to fully cover the plot. Therefore, in this experiment, all LiDAR data was partitioned into three groups according to the scan zenith angle of each point as follows: nadir $\left(0^{\circ}-7^{\circ}\right)$, small off-nadir $\left(7^{\circ}-23^{\circ}\right)$, and large off-nadir $\left(23^{\circ}-38^{\circ}\right)$, where each of the three groups have approximately comparable pulse numbers. All $P_{\text {gap }}$ and vertical $P_{\text {gap }}$ profiles were then calculated from each sub point cloud.

It is worth pointing out that for the same forest plot, the point density may change with different scan angles, depending on scanning parameters and the underneath surface. An ideal comparison would be done when pulse density is similar. It is possible to use pulse thinning to make point clouds of different scan angles more comparable. But the thinning procedure would bring more uncertainty. Therefore, raw data without thinning was used in this research. In another unpublished experiment by us, when pulse density was greater than $5 \mathrm{pl} / \mathrm{m}^{2}, P_{\text {gap }}$ was barely affected by increasing pulse density. 
To evaluate the accuracy of angular $P_{\text {gap }}$ derived from airborne LiDAR, the $P_{\text {gap }}$ calculated from DHP was used as the validation reference. DHP is one of the few equivalent techniques which can be used to validate $P_{\text {gap }}$ from airborne LiDAR and has been used in previous research (Hopkinson and Chasmer, 2007; Zheng et al., 2017). Correspondingly, the $P_{\text {gap }}$ derived from DHP was also averaged for nadir $\left(0^{\circ}-7^{\circ}\right)$, small off-nadir $\left(7^{\circ}-23^{\circ}\right)$ and large off-nadir $\left(23^{\circ}-38^{\circ}\right)$ direction. Simple linear regression analysis was then performed between the angular $P_{\text {gap }}$ from LiDAR and angular $P_{\text {gap }}$ from DHP, respectively for each direction. Unlike $P_{\text {gap }}$, the accuracy of vertical $P_{\text {gap }}$ profile is very difficult to evaluate. The most reliable approach might be to measure the $P_{\text {gap }}$ at each height level above ground. But this is too labor intensive and impractical in the field. As a result, the accuracy of vertical $P_{\text {gap }}$ profile was not validated.

To evaluate the scan angle impact, results of $P_{\text {gap }}$ and vertical $P_{\text {gap }}$ profile from nadir $\left(0^{\circ}-7^{\circ}\right)$, small off-nadir $\left(7^{\circ}-23^{\circ}\right)$, and large off-nadir $\left(23^{\circ}\right.$ $38^{\circ}$ ) directions were compared. Only sub point clouds that fully covered the plot were chosen for comparison, so as to achieve an objective and unbiased comparison. If a sub point cloud only sampled part of the plot, it was removed from further analysis. Paired-sample $t$-test between $P_{\text {gap }}$ from nadir, small off-nadir and large off-nadir directions were conducted, to test if there is a statistical significant difference. The null hypothesis was "there is no difference between the two $P_{\text {gap }}$ values from two scan angles". The alternative hypothesis was "there is difference between the two $P_{\text {gap }}$ value from two scan angles". The two-sided level of significance was $p<0.05$. When the calculated $t$ value is greater than the critical value and $p$ value is lower than 0.05 , the null hypothesis was rejected and conclude there is a difference in the two scan angles. Vertical $P_{g a p}$ profiles from nadir, small off-nadir, and large off-nadir directions in the same plot were first visually compared. To test whether scan angle has a significant impact on the derived vertical $P_{\text {gap }}$ profiles, the twotailed Mann Whitney U test was then conducted on the two vertical $P_{g a p}$ profiles from two scan angles, in each plot. The reason the two-tailed Mann Whitney U test was chosen was that the sample sets of each vertical $P_{\text {gap }}$ profile are not normally distributed. The null hypothesis was "there is no difference between the two vertical $P_{\text {gap }}$ profiles from two scan angles". The alternative hypothesis was "there is difference between the 
two vertical $P_{\text {gap }}$ profiles from two scan angles". The two-sided level of significance was $p<0.05$. When calculated $p$ value is lower than 0.05 , the null hypothesis was rejected and conclude there is a difference in the two vertical $P_{\text {gap }}$ profiles.

The quantification of scan angle impact on $P_{\text {gap }}$ was represented using $P_{\text {gap }}$ difference, which was the difference between every two $P_{\text {gap }}$ values from two different directions of the same plot. A higher $P_{g a p}$ difference indicated higher scan angle impact for $P_{\text {gap }}$ in this plot. To quantify the scan angle impact on vertical $P_{g a p}$ profiles, the root mean square difference (RMSD) between every two $P_{g a p}$ profiles was used. A higher RMSD indicates higher scan angle impact on vertical $P_{\text {gap }}$ profiles in this plot. In addition, the correlation coefficient was calculated between $P_{g a p}$ difference and nadir $P_{\text {gap }}$, and between RMSD of $P_{\text {gap }}$ profiles and nadir $P_{\text {gap }}$. A positive close to 1 correlation coefficient would suggest a strong positive correlation between the severity of scan angle impact and the nadir $P_{\text {gap }}$ in the plot.

\section{Results}

\section{1 $P_{\text {gap }}$ derived from DHP in different zenith angles}

For the 30 plots in the fieldwork, $P_{\text {gap }}$ derived from DHP at zenith angle from $0^{\circ}$ to $60^{\circ}\left(2.5^{\circ}\right.$ interval) for each plot is presented (Fig 4.3). For the coniferous plots, the observed $P_{\text {gap }}$ was very sensitive to zenith angle. A rapid decrease trend can be seen from $0^{\circ}$ to $20^{\circ}$. The $P_{\text {gap }}$ value was higher than 0.5 in $0^{\circ}$ nadir direction but decreased to lower than 0.3 at $60^{\circ}$ direction. The rapid decrease of $P_{\text {gap }}$ was also apparent in the open coniferous plots, but at a higher zenith angle ranging from $35^{\circ}$ to $60^{\circ}$. Unlike coniferous plots, in deciduous beech plots, the observed $P_{\text {gap }}$ was relatively insensitive to zenith angle in the range of $0^{\circ}$ to $60^{\circ}$, except one plot having a rapid decrease of $P_{\text {gap }}$ from $0^{\circ}$ to $10^{\circ}$. The decrease of $P_{\text {gap }}$ from $0^{\circ}$ to $60^{\circ}$ direction was usually less than 0.15 in deciduous plots. In mixed plots, more uncertainty and variability occurred. Change of $P_{\text {gap }}$ with zenith angle was pronounced in some mixed plots, but marginal in others. 


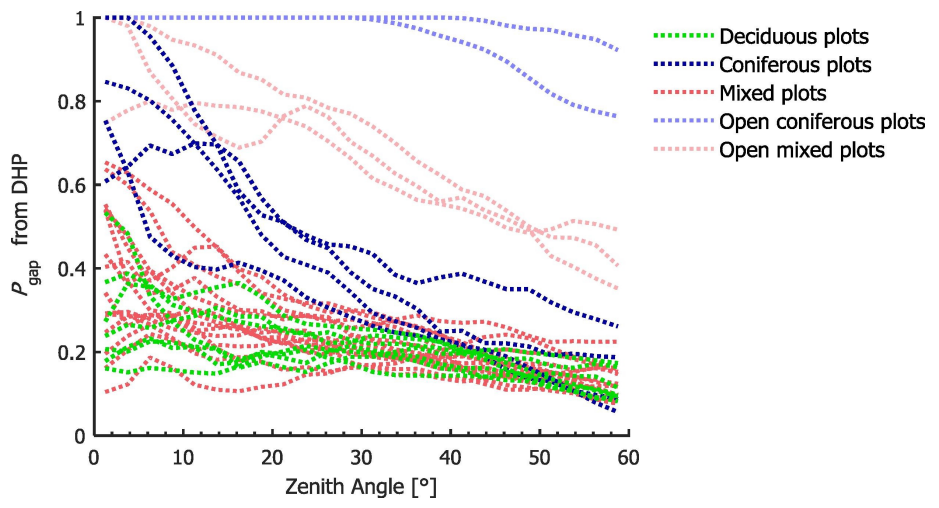

Figure 4.3: $P_{\text {gap }}$ of different angles measured from DHP at ground in deciduous, coniferous and mixed forest plots

\section{2 $P_{\text {gap }}$ derived from LiDAR data of different scan angles}

For all the 107 plots, after partition based on the scan angle, 321 datasets were generated. Each sub point cloud was examined to see if it could fully cover the plot. As a result, 26 plots were fully covered by all nadir $\left(0^{\circ}-7^{\circ}\right)$, small off-nadir $\left(7^{\circ}-23^{\circ}\right)$, and large off-nadir $\left(23^{\circ}-38^{\circ}\right)$ LiDAR data. 31 plots were fully covered by nadir and small off-nadir LiDAR data. 29 plots were fully covered by nadir and large off-nadir LiDAR data. 31 plots were fully covered by small off-nadir and large off-nadir LiDAR data. Subsequently, paired-sample $t$-test results between $P_{\text {gap }}$ from different scan angles are presented in Table 4.2. From the results, $P_{\text {gap } 0-7}$ is significantly higher than $P_{\text {gap } 7-23}(t=6.035>1.671, p<0.01)$, and $P_{\text {gap } 7-23}$ is further significantly higher than $P_{\text {gap }}>23(t=7.888>1.671, p<0.01)$. This indicates that $P_{\text {gap }}$ is maximum when measured by airborne LiDAR at the nadir direction, and observed $P_{\text {gap }}$ decreases with increasing LiDAR scan angle.

Table 4.2: Paired-sample $t$-test between $P_{\text {gap }}$ derived from LiDAR data of different scan angles

\begin{tabular}{cclcc}
\hline & Number of plots & $\begin{array}{l}\text { Degrees of } \\
\text { freedom (v) }\end{array}$ & $t$ & $p$ \\
\hline$P_{\text {gap } 0-7}, P_{\text {gap } 7-23}$ & 31 & 60 & 6.035 & $<0.001$ \\
$P_{\text {gap } 0-7}, P_{\text {gap }>23}$ & 29 & 56 & 7.846 & $<0.001$ \\
$P_{\text {gap } 7-23}, P_{\text {gap }>23}$ & 31 & 60 & 7.888 & $<0.001$ \\
\hline
\end{tabular}

$P_{\text {gap } 0-7}:$ gap fraction measured from LiDAR when scan angle ranges in $0^{\circ}-7^{\circ}$

$P_{\text {gap } 7-23}$ : gap fraction measured from LiDAR when scan angle ranges in $7^{\circ}-23^{\circ}$

$P_{\text {gap }}>23$ : gap fraction measured from LiDAR when scan angle exceeds $23^{\circ}$

critical value of $t$ at 0.05 significance level: $1.671(\mathrm{v}=60)$, and $1.673(\mathrm{v}=56)$ 


\subsection{Vertical $P_{\text {gap }}$ profile derived from LiDAR data with different scan angles}

Since different $P_{g a p}$ changes with the zenith angle can be seen among different forest types in section 3.1, the results of vertical $P_{g a p}$ profiles from different scan angles are also presented respectively for the deciduous, coniferous and mixed stands.
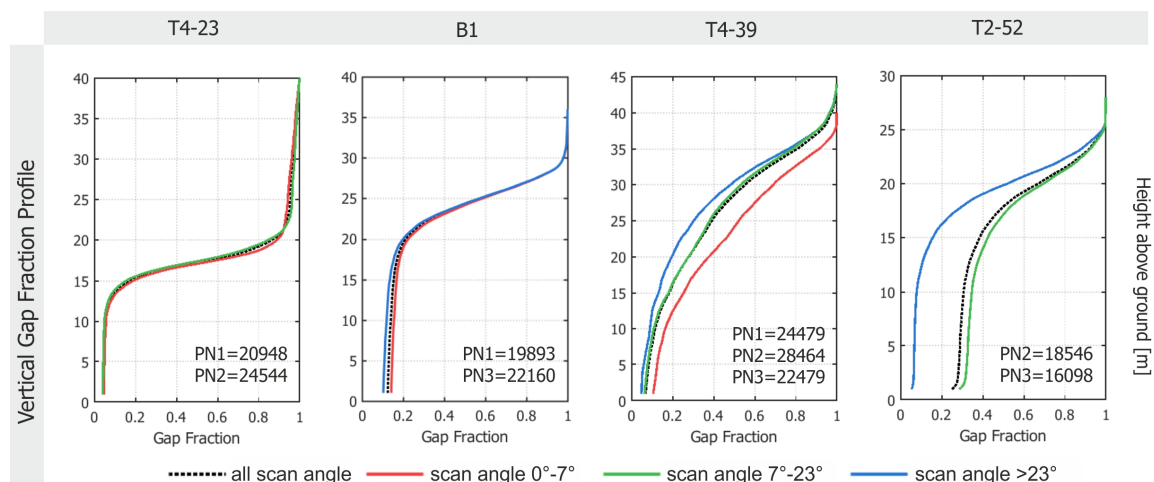

PN1: pulse number $\left(0^{\circ}-7^{\circ}\right) \quad$ PN2: pulse number $\left(7^{\circ}-23^{\circ}\right) \quad$ PN3: pulse number $\left(>23^{\circ}\right)$
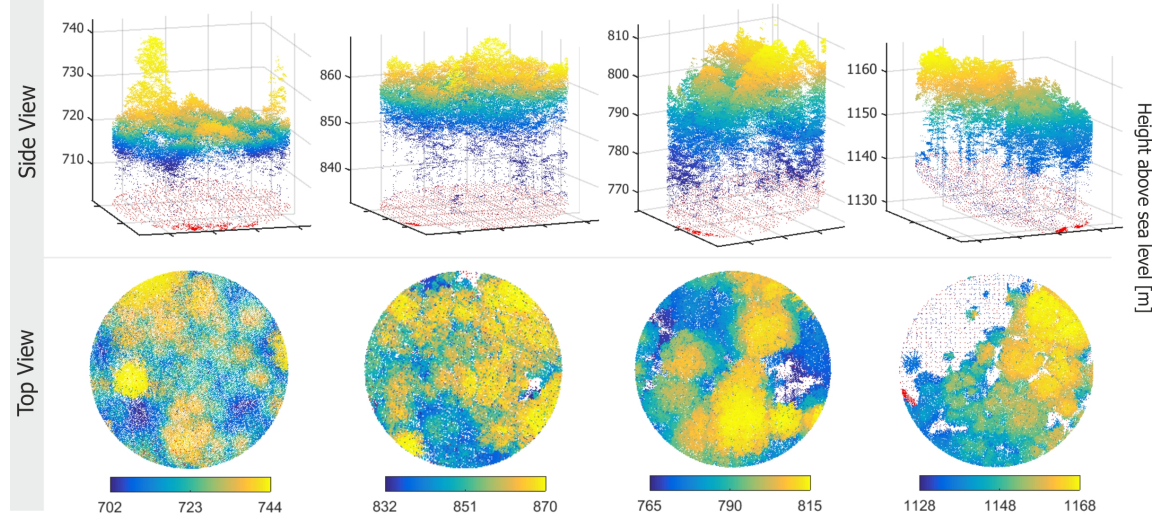

Figure 4.4: Vertical $P_{\text {gap }}$ profiles derived from airborne LiDAR data of different scan angles in deciduous beech plots (red points: ground returns). Note: there is one giant coniferous tree in plot T4-23

From Fig 4.4, in deciduous beech plots, vertical $P_{\text {gap }}$ profiles are almost the same from different scan angles in plot T4-23 ( $p=0.88$ ), where beech trees are very dense and there are few between-crown gaps. However, as the between-crown gaps begin to increase in plot B1, T4-39 and T252, vertical $P_{\text {gap }}$ profiles from different scan angles begin to diverge ( $p<0.01$ for B1, T4-39, and T2-52). At each height above ground, the 
observed $P_{\text {gap }}$ is always higher at small scan angle than at large scan angle. The height of $P_{\text {gap }}$ divergence occurs at approximately the same height as the densest position of the canopy. When it approaches the ground, $P_{\text {gap }}$ difference between scan angles are constant because of little understory and fewer laser interception. The shape of the vertical $P_{\text {gap }}$ profile is almost the same.

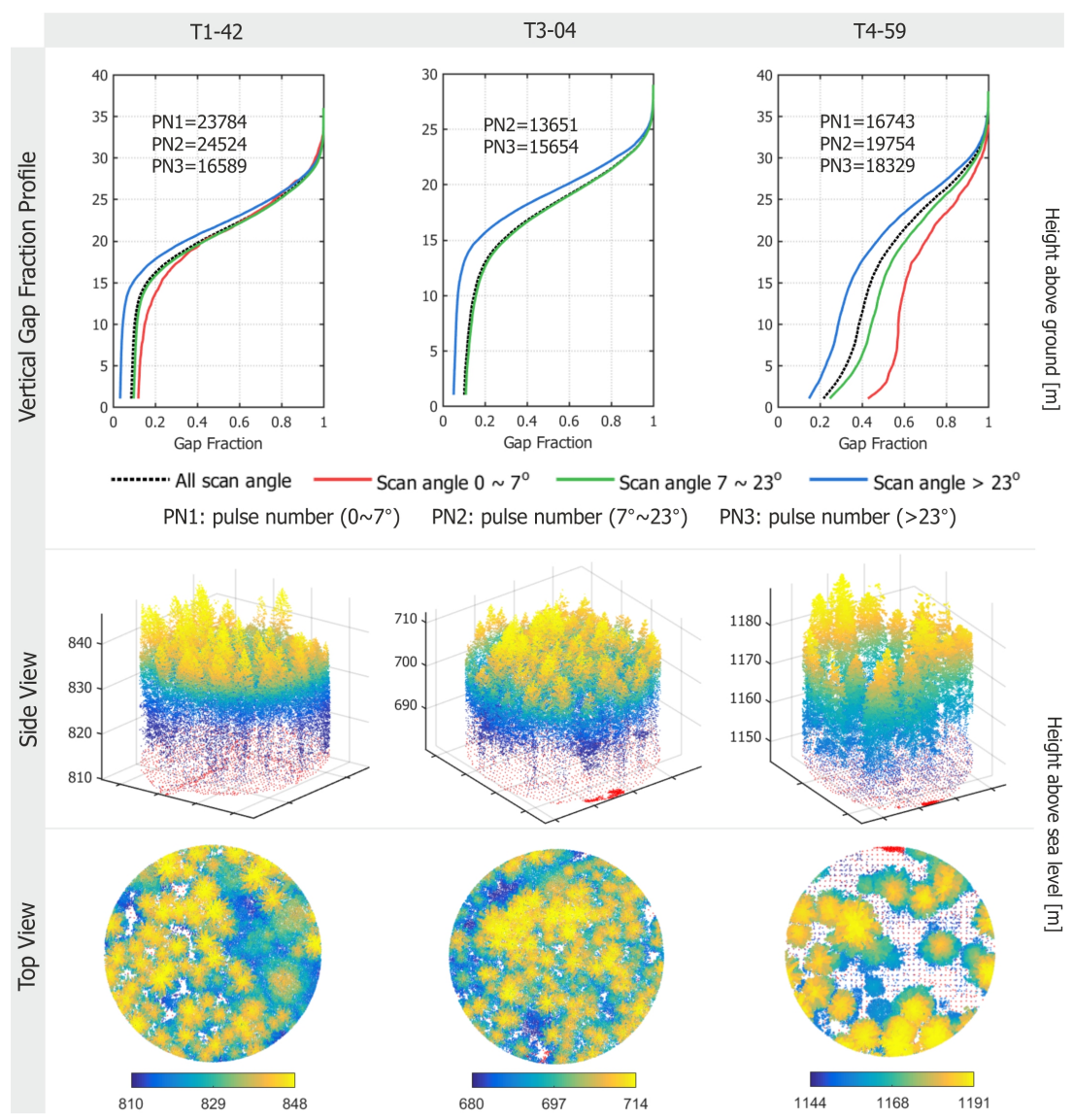

Figure 4.5: Vertical $P_{g a p}$ profiles derived from airborne LiDAR data of different scan angles in coniferous spruce plots (red points: ground returns)

Unlike the deciduous plots, in the coniferous plots, whether there are dense trees or sparse trees, $P_{\text {gap }}$ profiles are always sensitive to LiDAR scan angle (Fig 4.5). Even in a mature and dense plot as T1-42, the vertical $P_{\text {gap }}$ profile at large off-nadir direction differs greatly from the vertical $P_{\text {gap }}$ profile at nadir $(p<0.01)$. At each height below $15 \mathrm{~m}, P_{\text {gap }}>23$ is 
less than half of $P_{\text {gap } 0-7}$. Similar to deciduous plots, the difference of vertical $P_{\text {gap }}$ profiles increases when there are more between-crown gaps in the plot, such as T4-59 $(p<0.01)$. The shape of vertical $P_{g a p}$ profiles also changed.

Results of vertical $P_{\text {gap }}$ profiles in the mixed plots are presented in Fig 4.6. The general trend of increasing vertical $P_{\text {gap }}$ profile difference with increasing between-crown gaps can still be observed, for instance the large difference between vertical $P_{\text {gap } 7-23}$ profile and vertical $P_{g a p}>23$ profile in plot 3ad02 $(p<0.01)$. When the tree density is high in the plot T4-14, the vertical $P_{\text {gap }}$ profile change is marginal $(p=0.48)$. But in another dense plot T4-21, vertical $P_{\text {gap }}$ profiles are quite different from different scan angles $(p<0.01)$.
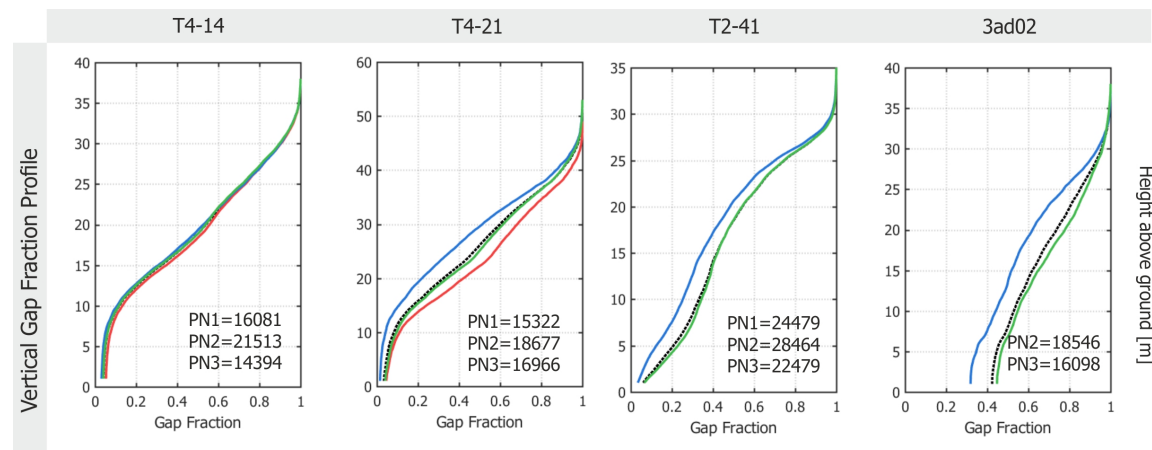

........ All scan ang

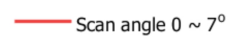

Scan angle $7 \sim 23^{\circ}$

Scan angle $>23^{\circ}$ PN1: pulse number $\left(0 \sim 7^{\circ}\right) \quad$ PN2: pulse number $\left(7^{\circ} \sim 23^{\circ}\right) \quad$ PN3: pulse number $\left(>23^{\circ}\right)$
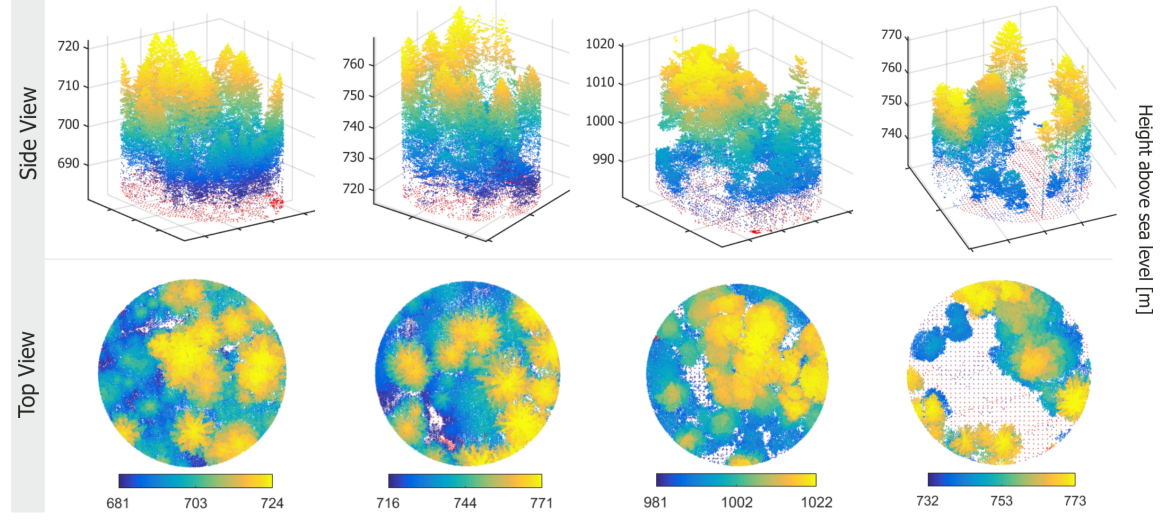

Figure 4.6: Vertical $P_{\text {gap }}$ profiles derived from airborne LiDAR data of different scan angles in mixed plots (red points: ground returns) 


\subsection{Statistical analysis}

\subsubsection{Agreement of $P_{\text {gap }}$ derived from DHP and LiDAR}

Without considering scan angle, angle-averaged $P_{\text {gap }}$ derived from LiDAR and from DHP are correlated well with each other (Fig 4.7 a)). When considering scan angle, the correlation between angular DHP $P_{\text {gap }}$ and LiDAR $P_{\text {gap }}$ is best when scan angle ranges from $7^{\circ}-23^{\circ}$ (Fig $4.7 \mathrm{c}$ )), and worst when scan angle exceeds $23^{\circ}$ (Fig $4.7 \mathrm{~d}$ )). Regardless of the scan angle, angular LiDAR $P_{\text {gap }}$ is always lower than angular DHP $P_{\text {gap }}$. This effect is most obvious for large off-nadir scan angles $\left(>23^{\circ}\right)$. In nadir direction $\left(0^{\circ}-7^{\circ}\right)$, plots with low LiDAR $P_{\text {gap }}$ have much higher DHP $P_{\text {gap }}$, while plots with high LiDAR Pgap have high DHP $P_{g a p}$ as well (closer to the $1: 1$ line).
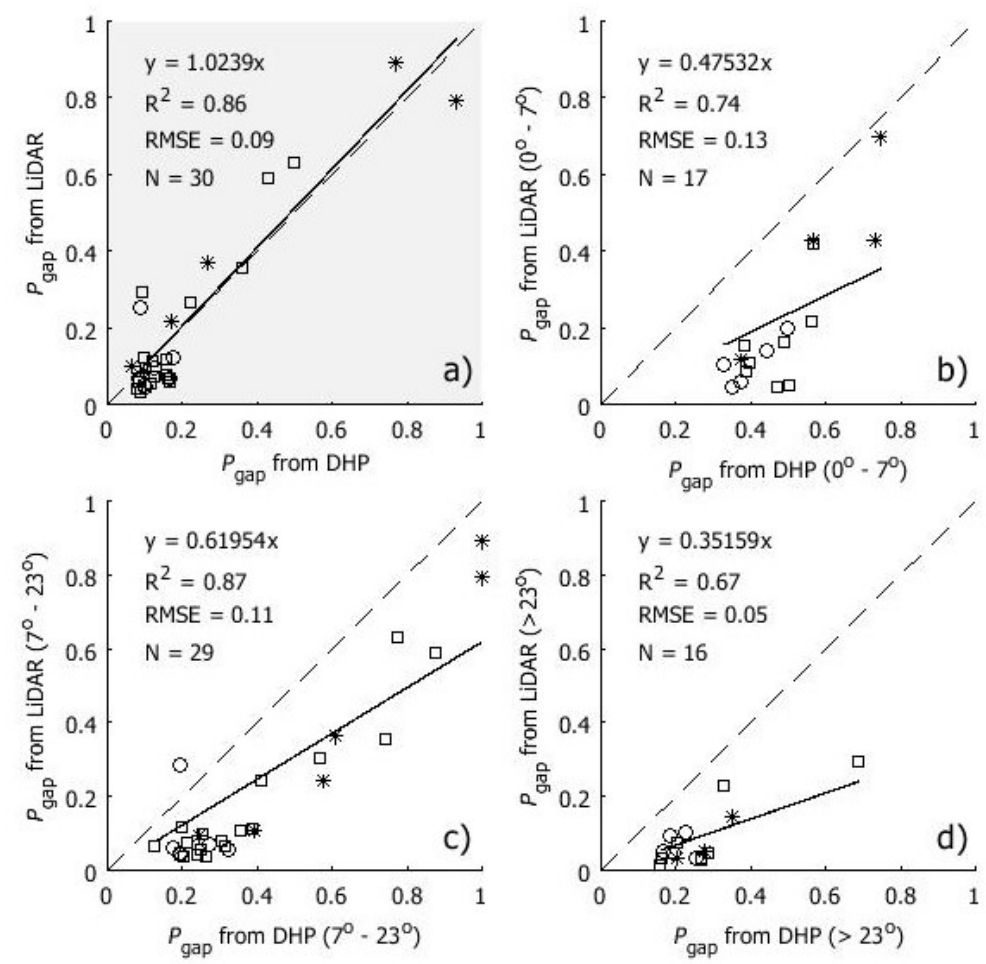

coniferous plots

deciduous plots

․ mix plots

Figure 4.7: Correlation between the $P_{\text {gap }}$ derived from DHP and the $P_{g a p}$ derived from airborne LiDAR. a) without considering scan angle; b) scan angle ranges from $0^{\circ}-7^{\circ}$; c) scan angle ranges from $7^{\circ}-23^{\circ}$; d) scan angle exceeds $23^{\circ}$. In each sub figure, $\mathrm{N}$ is the number of plots 


\subsubsection{Correlation between nadir $P_{\text {gap }}$ and scan angle impact on $P_{\text {gap }}$, vertical $P_{\text {gap }}$ profile}

Results in Section 3.3 indicate that the scan angle effect differed between deciduous beech plots and coniferous spruce plots. Furthermore, the between-crown gaps can lead to large $P_{\text {gap }}$ difference. We assume that plots with more within-crown gaps or between-crown gaps will be more sensitive to LiDAR scan angle. Therefore, the correlation between nadir $P_{\text {gap }}$ and scan angle impact is explored.

Scan angle impact on $P_{\text {gap }}$ above ground level is quantified using $P_{\text {gap }}$ difference. A higher $P_{\text {gap }}$ difference means observing the plot in different LiDAR scan angles will end up in quite different $P_{\text {gap }}$ values. Using the 91 paired-samples in Table 4.2, the correlation between $P_{\text {gap }}$ difference and the nadir $P_{\text {gap }}$ in the plot is explored. From Fig 4.8, it is clear that there is a strong positive correlation $(r=0.71, p<0.001)$ between nadir $P_{\text {gap }}$ and $P_{\text {gap }}$ difference.

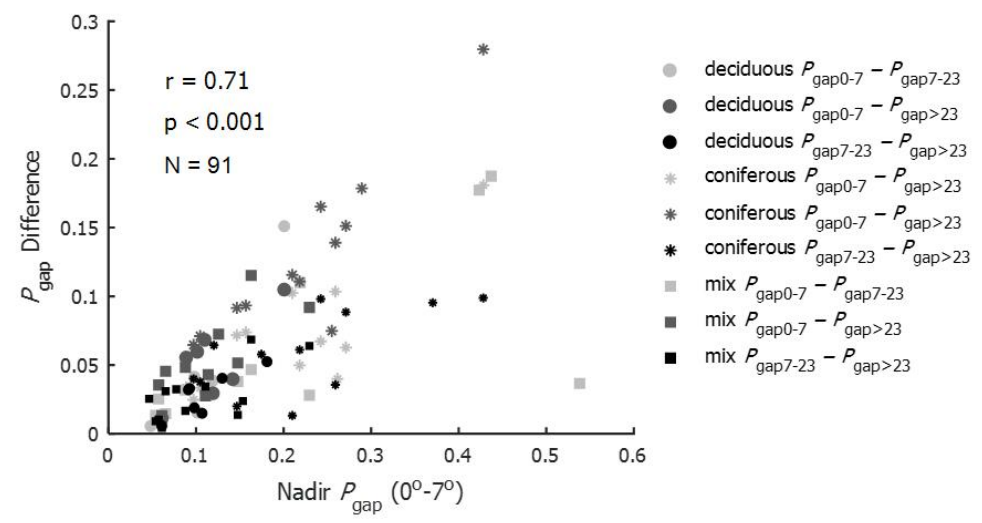

Figure 4.8: Correlation between nadir $P_{\text {gap }}$ and the difference of $P_{g a p}$ measured from airborne LiDAR of different scan angles in deciduous, coniferous and mix forest plots. $\left(P_{\text {gap } 0-7}, P_{\text {gap } 7-23}\right.$ and $P_{\text {gap }>23}$ : gap fraction measured from LiDAR when scan angle ranges in $0^{\circ}-7^{\circ}, 7^{\circ}-23^{\circ}$, greater than $23^{\circ}$ respectively)

Scan angle effect on vertical $P_{\text {gap }}$ profile is quantified using the RMSD between two vertical $P_{\text {gap }}$ profiles derived from the same plot observed by LiDAR from two different scan angles. A higher RMSD value suggests large difference between the two vertical $P_{\text {gap }}$ profiles and large scan angle impact. From Fig 4.9, a moderate positive correlation ( $r=0.66$, 
$p<0.001)$ is observed between nadir $P_{\text {gap }}$ and the RMSD of vertical $P_{\text {gap }}$ profiles. This is a similar trend as shown in Fig 4.8. Scan angle impact on the vertical $P_{\text {gap }}$ profile is also more severe in plots with more gaps, and less important in plots with fewer gaps. But the distribution of points are more spread than in Fig 4.8.

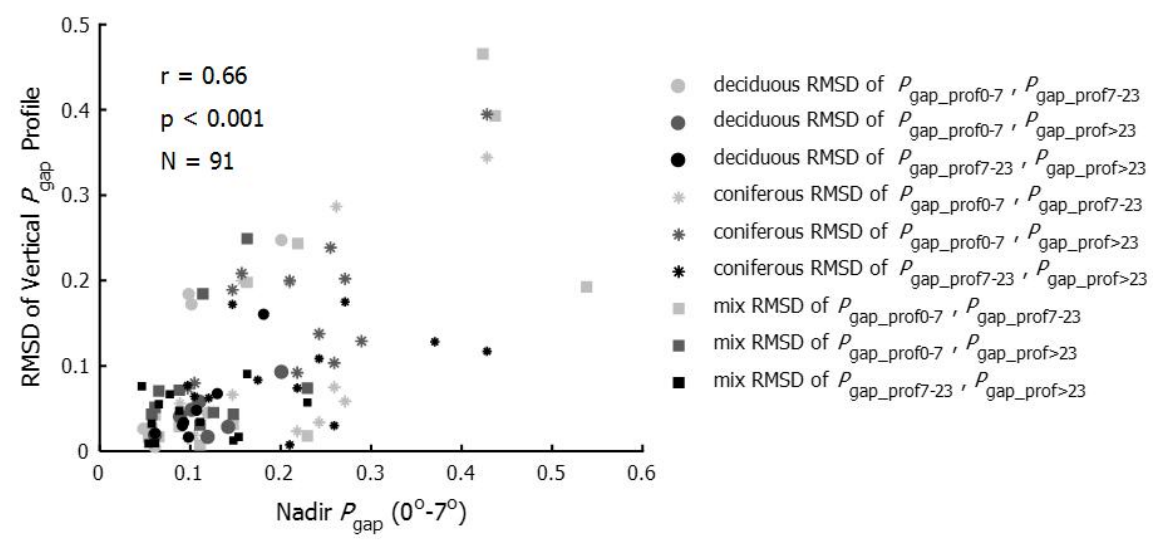

Figure 4.9: Correlation between nadir $P_{\text {gap }}$ and the RMSD of each two vertical $P_{\text {gap }}$ profiles derived from airborne LiDAR of two different scan angles, in deciduous, coniferous and mix forest plots. $\left(P_{\text {gap_prof0- }}\right.$, $P_{\text {gap_prof7-23 }}$ and $P_{\text {gap_prof }>23}$ : vertical $P_{\text {gap }}$ profiles measured from LiDAR when scan angle ranges in $0^{\circ}-7^{\circ}, 7^{\circ}-23^{\circ}$, greater than $23^{\circ}$ respectively)

\section{Discussion}

\subsection{Agreement of $P_{\text {gap }}$ derived from DHP and LiDAR}

Without considering scan angle, the results suggest a good agreement between angle-averaged LiDAR $P_{\text {gap }}$ and DHP $P_{\text {gap }}$ (Fig 4.7 a)). When considering scan angle, generally a high correlation between angular LiDAR $P_{\text {gap }}$ and DHP $P_{\text {gap }}$ can be observed for all scan angles (Fig 4.7 b), c), d)). This demonstrates the potential of airborne LiDAR to accurately map angular $P_{\text {gap }}$ in different forest types. Compared to DHP, which often have a higher variance and bias, airborne LiDAR is relatively more stable.

It is also noteworthy that airborne LiDAR $P_{g a p}$ is always lower than DHP $P_{\text {gap }}$, at all scan angles ( $0^{\circ}-38^{\circ}$ in this research). This closely matches 
earlier findings when using terrestrial LiDAR (Hancock et al., 2014; Seidel et al., 2012). Furthermore, comparing different scan angles, the underestimation of $P_{\text {gap }}$ becomes more severe for large scan angle LiDAR (Fig $4.7 \mathrm{~d}$ )). The most likely reason appears to be the large footprint of airborne laser pulse and lower sensitivity of the laser sensor. Unlike DHP, which can be very sensitive to a ray of light penetrating small canopy gaps, laser pulses have a much larger footprint, lower energy density in the footprint, and higher backscattered energy threshold to trigger the recording of each return. Therefore, when a laser pulse penetrates small gaps, the backscattered energy might be too low to trigger the laser sensor to start recording the return. Even when the sensor is triggered, these low amplitude energy spikes may not be preserved in the decomposition processing from the raw waveform data. The loss of these small gaps may be the main reason causing underestimation of LiDAR $P_{g a p}$ compared to DHP $P_{\text {gap }}$. With scan angle increasing, a longer path for the laser pulse penetrating the canopy as well as more challenging detection of small gaps lead to more severe underestimation of $P_{\text {gap }}$.

Overall, airborne LiDAR provides the potential for mapping angular $P_{g a p}$ over a large extent, but underestimation often occurs and is magnified by an increasing scan angle. For this reason, it is recommended to avoid using large off-nadir scan angle $\left(>23^{\circ}\right)$ when estimating $P_{\text {gap }}$.

\subsection{Scan angle impact on $P_{g a p}$ in different forest types}

From the results of this research, the scan angle impact on $P_{\text {gap }}$ differs among different forest types.

In coniferous spruce plots, scan angle has a large impact on the measured $P_{\text {gap }}$, both from airborne LiDAR (Fig 4.5) and from DHP (Fig 4.3). This demonstrates the anisotropy of $P_{\text {gap }}$ in coniferous spruce plots. From $0^{\circ}-20^{\circ}$, the $P_{\text {gap }}$ decreases rapidly with increasing scan angle (Fig 4.3 and Fig 4.5). Maximum $P_{\text {gap }}$ is observed in nadir direction. This finding is consistent with previous research in a mountain pine forest using terrestrial LiDAR and DHP (Danson et al., 2007), and in a lodgepole pine plot (Canham et al., 1990) also using DHP. This rapid decrease of $P_{g a p}$ to scan angle in coniferous plots could be explained by the tall conical crown shape and frequent between-crown openings in these forests 
(Canham et al., 1990).

Unlike in coniferous plots, scan angle has a negligible impact in dense deciduous beech forest (i.e. deciduous forests behave as a isotropic surface). For example, the $P_{\text {gap }}$ value at ground level is relatively insensitive to scan angle in plots T4-23 and B1 (Fig 4.4). A possible reason may be the different canopy architecture due to leaf shape and leaf angle, between deciduous and coniferous forest types. The sensitivity of $P_{g a p}$ with scan angle in spruce plots, appears similar to the spherical or erectophile leaf angle distribution case (Fig 4.10) (de Wit, 1965), implying the spruce leaves and shoots grow more vertically than horizontally. This can be observed from Fig 4.1 b. While in dense beech plots, stability of $P_{\text {gap }}$ to scan angle appears similar to the planophile or plagiophile leaf angle distribution case (de Wit, 1965) (Fig 4.10), when beech leaves tend to grow more horizontally than vertically and have small inclination angles. This can also be observed from Fig 4.1 a. Other sources of the difference between coniferous and deciduous plots may be the anisotropy of clumping index, i.e., $\lambda$ is not constant in different scan angles (Rautiainen and Stenberg, 2015). However, when between-crown gaps arise in deciduous plots, the scan angle has a substantial impact (for example, the $P_{g a p}$ value for plots T4-39 and T2-52 in Fig 4.4). This appears similar to the findings in Piayda et al. (2015), where measured $P_{\text {gap }}$ decreased from 0.8 at nadir to 0.5 at $60^{\circ}$ angle, in a savanna-type cork oak forest.

A further useful finding from this research is the strong positive correlation $(r=0.71, p<0.001)$ between nadir $P_{\text {gap }}$ and $P_{\text {gap }}$ difference (Fig 4.8). This supports the previous assumption, that LiDAR scan angle impact on $P_{\text {gap }}$ is more severe in plots with more gaps (either within-crown or between-crown gaps), less important in plots with few gaps. It also offers an easy and straight-forward approach to predict how much the $P_{g a p}$ value of a plot is impacted by scan angle. If a plot is more "patchy", such as discontinuous coniferous plots or young sparse deciduous plantation, it can be predicted that the nadir $P_{\text {gap }}$ will be quite different from (higher than) off-nadir $P_{\text {gap }}$ using LiDAR. Although there is no directly related LiDAR research, using DHP method, measured $P_{\text {gap }}$ was found to be sensitive to and decreasing with increasing zenith angle in five deciduous plots after selection cutting (Beaudet and Messier, 2002). In contrast, 


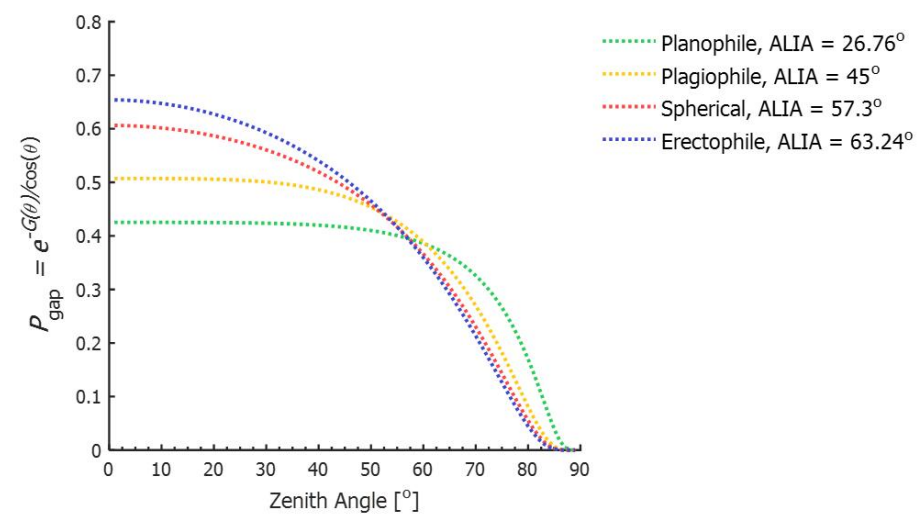

Figure 4.10: Theoretical $P_{\text {gap }}$ value change with zenith angle using predefined planophile, plagiophile, spherical and erectophile leaf angle distribution (de Wit 1965), when LAI $=1$ and $\lambda=1$ (ALIA: average leaf inclination angle)

measured $P_{\text {gap }}$ was much more stable to zenith angle in 5 paired control plots without cutting (Beaudet and Messier, 2002).

Statistical results in Table 4.2 suggests sensitivity to LiDAR scan angle is the trend for most plots in this research. This may be caused by the widespread gap existence in this study area, at $20 \mathrm{~m}$ radius plot level (Lausch et al., 2013). Canopy gaps are quite common in woodland ecosystems due to tree spacing, blowdowns, tree mortality and other factors (Canham et al., 1990; Asner, 1998). Especially in this study area, in order to protect wildlife biodiversity and the natural dynamic process in the park, managers employed a non-intervention strategy in at least 75\% of the park (Heurich et al., 2011). Wind-throw and bark beetle infestation also adds to the formation of gaps.

\subsection{Scan angle impact on the vertical $P_{\text {gap }}$ profile in different forest types}

The capability of mapping large extent vertical $P_{\text {gap }}$ profile is a major advantage of airborne LiDAR, compared to other passive optical sensors, ground-based DHP method, or terrestrial LiDAR. However, the results in this study has demonstrated the sensitivity and possible large difference of vertical $P_{\text {gap }}$ profiles from different LiDAR scan angles. 
Similar to $P_{\text {gap }}$, the vertical $P_{\text {gap }}$ profile is also more sensitive to LiDAR scan angle in plots with more gaps such as discontinuous coniferous plots and deciduous plots with between crown gaps (Fig 4.9). At all scan angles, vertical $P_{\text {gap }}$ profile from LiDAR is decreasing monotonically from canopy surface down to ground, due to longer path of interception by photosynthetic and non-photosynthetic elements. But when analyzing at each height level, there is much uncertainty regarding how much the difference is between $P_{\text {gap }}$ from different scan angles. For instance, in beech plot B1 and T2-52, vertical $P_{\text {gap }}$ profiles are increasingly diverging from canopy top to ground (Fig 4.4). But in plot T4-39, vertical $P_{\text {gap }}$ profiles first diverge from canopy top to $25 \mathrm{~m}$ above ground, and then start converging to each other. Without ground truth data on vertical $P_{\text {gap }}$ profiles, it is not clear what causes this variability, perhaps due to the vertical heterogeneity of leaf area density, leaf spatial distribution and leaf angle inclination at different height levels. Further study could investigate this with the ancillary terrestrial LiDAR data. When in mix plots, the uncertainty in vertical $P_{\text {gap }}$ profiles' divergence and convergence happens more, as shown in plot T4-21, T2-41 and 3ad02 (Fig 4.6). There is a high probability that the mixing of different species and canopy architectures adds to the vertical structure heterogeneity. This may lead to the uneven scan angle effect in different height levels.

\subsection{Implications}

First of all, LiDAR derived $P_{\text {gap }}$ accuracy changes with scan angles. This result can provide some guidance on LiDAR flight design for LAI mapping. A growing number of studies have applied airborne LiDAR to map LAI (Riaño et al., 2004; Morsdorf et al., 2006; Solberg et al., 2009; Alonzo et al., 2015). The gap fraction method was shown to be more accurate and robust than allometric methods (Richardson et al., 2009). Therefore, an accurate measurement of $P_{g a p}$ is critical to accurate estimation of LAI. According to results in 3.4.1, when the LiDAR scan angle strongly deviates from $0^{\circ}$, the agreement between LiDAR $P_{\text {gap }}$ and DHP $P_{\text {gap }}$ became worse (Fig $4.7 \mathrm{~d}$ )). This implies that further LAI mapping should avoid LiDAR flight at large off-nadir scan angles. Because of the limited number of plots with DHP, a threshold scan angle cannot be recommended and 
further research is required to investigate a threshold scan angle to meet a pre-defined (required) LAI accuracy.

Second, the results of this study demonstrated that scan angle has different impacts on $P_{\text {gap }}$ and vertical $P_{\text {gap }}$ profiles in coniferous, deciduous and mix plots. This leads us to question the commonly used spherical leaf angle distribution assumption in previous research (Richardson et al., 2009; Tang et al., 2012). On one hand, a small scan angle is preferred to achieve more accurate $P_{\text {gap }}$. On the other hand, a small scan angle is far from the $57.5^{\circ}$ angle, when the leaf projection function $G(\theta)$ can be considered as almost independent on leaf inclination $(G(\theta)=0.5)$ (Wilson, 1963). This implies special consideration and estimation of $G(\theta)$ has to be taken for more accurate LAI estimation. Some researchers have estimated $G(\theta)$ from ground collected DHP (Korhonen et al., 2011) or calibrated the airborne LAI model through regression of LiDAR $P_{\text {gap }}$ against ground measurements of LAI (Solberg et al., 2009). Another possible solution, would be direct retrieval of leaf angle distribution $g(\theta)$ and leaf projection function $G(\theta)$ from airborne LiDAR data (Ma et al., 2017).

The scan angle impact results on vertical $P_{g a p}$ profile, also points out the importance of correcting for scan angle effect in forest vertical structure analysis. For most research utilizing LiDAR to quantify forest vertical structure, vertical $P_{\text {gap }}$ profile is a prerequisite product, and then vertical LAI profile is resolved based on the Beer-Lambert law. In recent years, more quantitative metrics have emerged from the vertical LAI profile, including vertical distribution ratio (Goetz et al., 2007), canopy Shannon index (Stark et al., 2012), canopy strata (Wilkes et al., 2015a). Therefore, without correcting for scan angle impact on vertical $P_{\text {gap }}$ profiles, all the subsequent vertical LAI profile and derivative metrics may not be accurate or informative, especially when comparing datasets acquired from multiple LiDAR flights with different scan angles.

More broadly, $P_{\text {gap }}$ is one of the reasons causing canopy reflectance variability. For coarse spatial resolution instruments, the existence of within-crown and between-crown gaps expose understory and bare soil to passive sensors, and cause spectral mixture in the signal (Asner, 1998). The sensitivity of $P_{\text {gap }}$ to scan angle, leads to different portions of understory and soil background entering the sensor field of view. 
This may be one of the reasons causing anisotropy behavior of canopy reflectance, and angular dependence of vegetation indices (Myneni et al., 1995; Middleton et al., 2016), as well as biochemical property inversion results (Kempeneers et al., 2008). The potential of LiDAR to retrieve leaf projection function $G(\theta)$ and model $P_{\text {gap }}$ for any angle may help resolve the anisotropy behavior of canopy reflectance (especially for conifers), as well as correct the scan angle effect and refine the inversion of biophysical and biochemical properties from passive multispectral or hyperspectral data in the future.

\section{Conclusion}

In this research, the impact of off-nadir scan angle on $P_{g a p}$ and vertical $P_{\text {gap }}$ profile was evaluated, for several forest types. $P_{\text {gap }}$ and vertical $P_{\text {gap }}$ profile were first calculated from multi angle airborne LiDAR data. Digital hemispherical photographs (DHP) were then used as references for validation. The results showed that angular $P_{\text {gap }}$ from airborne LiDAR correlates well with angular $P_{g a p}$ from DHP. But underestimation of $P_{\text {gap }}$ from LiDAR amplifies at large off-nadir scan angle. The scan angle impact on $P_{g a p}$ and vertical $P_{g a p}$ profiles differed among different forest types. The impact was more severe for plots with discontinuous or sparse canopies. It was suggested that large off-nadir scan angle of airborne LiDAR should be avoided to ensure a more accurate $P_{g a p}$ and LAI estimation. The angular dependence of vertical $P_{\text {gap }}$ profiles should be accounted for, to improve the retrieval of LAI profiles, and other quantitative canopy structural metrics. 


\section{Topographic normalization effect on vertical LAI profile *}

*This chapter is based on: Liu, J., Skidmore, A., Heurich, M., Wang, T., 2017. Significant effect of topographic normalization of airborne LiDAR data on the retrieval of plant area index profile in mountainous forests. ISPRS Journal of Photogrammetry and Remote Sensing, 132, 77-87. 


\begin{abstract}
As an important metric for describing vertical forest structure, the vertical leaf area index (LAI) profile is used for many applications including carbon modelling and wildlife habitat assessment. Vertical LAI profiles can be estimated with the vertically resolved gap fraction from airborne LiDAR data. Most research utilizes a height normalization algorithm to retrieve local or relative height by assuming the terrain to be flat. However, for many forests this assumption is not valid. In this research, the effect of topographic normalization of airborne LiDAR data on the retrieval of vertical LAI profile was studied in a mountainous forest area in Germany. Results show that, although individual tree height may be retained after topographic normalization, the spatial arrangement of trees is changed. Specifically, topographic normalization vertically condenses and distorts the vertical LAI profile, which consequently alters the distribution pattern of leaf area density in space. This effect becomes more evident as the slope increases. Furthermore, topographic normalization may also undermine the complexity (i.e., canopy layer number and entropy) of the vertical LAI profile. The decrease in vertical LAI profile complexity is not solely determined by local topography, but is determined by the interaction between local topography and the spatial distribution of each tree. This research demonstrates that when calculating the vertical LAI profile from airborne LiDAR data, local topography needs to be taken into account. We therefore suggest that for ecological applications, such as vertical forest structure analysis and modeling of biodiversity, topographic normalization should not be applied in non-flat areas when using LiDAR data.
\end{abstract}




\section{Introduction}

A vertical leaf area index (LAI) profile is a quantitative description of how leaves are distributed vertically, and is expressed as a function of height (McElhinny et al., 2005; Bergen et al., 2009). In forest ecosystems, the vertical distribution of leaves influence several processes, such as radiation interception (Parker et al., 2001), patterns of infiltration and evapotranspiration (Farid et al., 2008), soil erosion (Nanko et al., 2008), and nutrient cycling (Tateno et al., 2004). In forest inventory, vertical LAI profiles have been successfully used to estimate variables such as biomass growth (Stark et al., 2012), canopy height (Lefsky et al., 1999), and basal area (Lefsky et al., 1999), all important in forest inventory and stand management. In forest ecology studies, vertical LAI profiles also play an important role. The vertical distribution of foliage is one of the most widely used variables in forest biodiversity research, especially in wildlife habitat modeling, such as for bird and bat species (MacArthur and MacArthur, 1961; Brokaw and Lent, 1999; Goetz et al., 2007; Vierling et al., 2008; Muller et al., 2013). Vertical LAI profiles have also been used for carbon stock modeling (Kotchenova et al., 2004; Lefsky et al., 2005; Shugart et al., 2010), and mapping of forest succession stages (Falkowski et al., 2009).

Canopy layering derived from a vertical LAI profile is a useful variable in forest ecology. Canopy layering refers to the clumped vertical distribution of vegetation within different height categories (Hollinger, 1989). The number of canopy layers is a categorical variable describing the complexity of forest vertical structure. It is a vital attribute that determines habitat quality and quantity for many forest-dwelling organisms (Allee et al., 1949; Franklin and Spies, 1991; Humphrey et al., 1999). In addition to canopy layering, the entropy of a vertical LAI profile is a continuous variable describing the complexity of the vertical forest structure. Some researchers have used Shannon's entropy to measure the vertical complexity of a forest (MacArthur and MacArthur, 1961; Treuhaft et al., 2009; Stark et al., 2012), and further used it to predict biodiversity (MacArthur and MacArthur, 1961). Large entropy values indicate a more continuous leaf area density across the profile (Stark et al., 2012). According to the niche differentiation concept, more species could then be supported 
through partitioned niche space (Tews et al., 2004). A larger entropy value may thus imply that more niches become available for biodiversity (Bergen et al., 2009).

Mapping vertical LAI profiles is beyond the capability of traditional airborne and spaceborne optical remote sensing techniques (Weishampel et al., 2000; Morsdorf et al., 2010). However, LiDAR technology has the advantage of estimating forest vertical structure (Hyde et al., 2005). Both waveform LiDAR (Harding et al., 2001; Lindberg et al., 2012; Tang et al., 2012) and discrete return LiDAR (Coops et al., 2007; Hilker et al., 2010) have been successfully used to calculate gap fraction $\left(P_{\text {gap }}\right)$ and LAI profiles. This has been generally conducted through estimates of the vertically resolved $P_{\text {gap }}$ from airborne LiDAR data. The cumulative LAI profile can then be estimated as a function of height from the vertical $P_{\text {gap }}$ profile based on the Beer's law (Lovell et al., 2003; Tang et al., 2012). The derivative of the cumulative LAI profile is then attained and serves as the vertical LAI profile.

In many previous studies, prior to calculating vertical LAI profiles from airborne LiDAR data, a topographic normalization was conducted (Coops et al., 2007; Hilker et al., 2010; Palace et al., 2015). A normalized point cloud is generated by subtracting the ground elevation from the original ellipsoidal height (sea level height) of each return. Thus, a local or relative height is established. This process is quite similar to the generation of normalized digital surface model (nDSM) or canopy height model (CHM), through subtracting a digital terrain model (DTM) from a digital surface model (DSM). Both the normalized point cloud or normalized raster image nDSM are representations of objects rising from the terrain approximately put on a plane (Haala and Brenner, 1999). However, local topographic change is neglected, and a flat local terrain assumption is implied for all the vegetation above. Unfortunately, in mountainous forests, this assumption is often not valid. Previous research has shown that neglecting topography will cause errors to LiDAR derived individual tree metrics such as canopy height (up to $1.78 \mathrm{~m}$ ) and tree top location (up to $1.80 \mathrm{~m}$ ), especially for trees with an irregular crown pattern and weak apical dominance (Khosravipour et al., 2015; Véga and Durrieu, 2011). To date, no research has been conducted examining how topographic normalization will affect the retrieval of vertical LAI profiles and 
its derived metrics.

An illustration of how topography affects the vertical leaf area distribution can be seen in Fig 5.1. Tree shape and height are consistent across all three plots, while the local topography as well as the spatial distribution of trees are different. Since the total vegetation remains consistent in all three plots, the total LAI of these three plots is the same. However, as the leaf area has different vertical distribution, the vertical LAI profiles are also different. In this case, the direct and diffuse radiation distribution, absorption of photosynthetically active radiation (PAR), and canopy reflectance in these plots are also different (Wang and Li, 2013). The dissimilar vertical distribution of direct radiation and diffuse radiation further leads to different gross primary production (GPP) accumulations and vertical biomass accumulation (Kotchenova et al., 2004). Therefore, ecologically, the three plots in Fig 5.1 have different radiation regimes and local climate. However, using topographic normalization, plot a) and b) will be normalized and become equivalent to plot c) regarding their vertical LAI profiles.

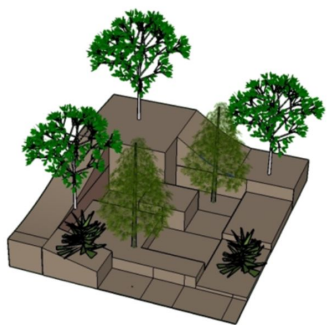

(a)

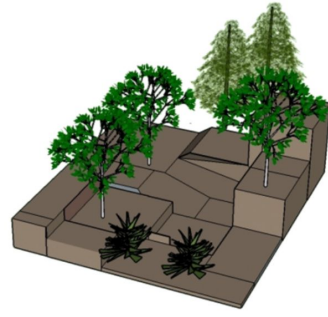

(b)

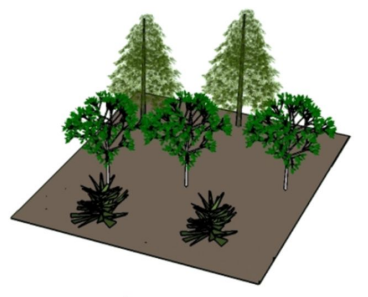

(c)

Figure 5.1: Schematic illustration of how local topography affects the vertical leaf area distribution. Three plots (a), (b), and (c) with the same trees and leaf area but different spatial distribution of leaves on different local topography conditions. Differences in the vertical structure of the three plots are eliminated after topographic normalization

The objectives of this chapter are to 1) determine the difference between vertical LAI profiles before and after topographic normalization of LiDAR data; 2) evaluate how the metrics derived from the vertical LAI profile (i.e., vertical extent, canopy layer number and entropy) change due to topographic normalization, and 3) explore the relationship between the degree of change and local topography. 


\section{Materials}

\subsection{Study Area}

The study area is located in the Bavarian Forest National Park, a temperate forest in southeastern Germany. This park was chosen as the study area due to its diverse forest structure and airborne LiDAR data availability. The park covers an area of $243 \mathrm{~km}^{2}$. The topography is largely comprised of gentle slopes, with the elevation ranging from about 600 to 1450 $\mathrm{m}$ (Fig 5.2). The main land cover classes include coniferous forests (young, medium, mature), deciduous forests (young, medium, mature), mixed forests (young, medium, mature), meadows (cultivated, natural, wetlands), lying deadwood, and standing deadwood areas. Dominant tree species in the study area are Norway spruce (Picea abies) (67\%) and European beech (Fagus sylvatica) (24.5\%). The study area encompasses a mixture of forest types with high structural complexity, Above 1100 $\mathrm{m}$ subalpine spruce forests are found containing Norway spruce and occasionally mountain ash (Sorbus aucuparia). Between $600 \mathrm{~m}$ and 1100 m, mature mountain mixed forests with Norway spruce, white fir (Abies alba), European beech and sycamore maple (Acer pseudoplatanus) occur. Wet depressions in the valleys harbor highly mixed forest with Norway spruce, mountain ash and birches (Betula spp.) (Heurich et al., 2010).

\subsection{Plot Selection}

Vertical LAI profiles are generally calculated and analyzed at plot level. In this study, 33 square plots of size $30 \mathrm{~m}$ by $30 \mathrm{~m}$ were selected from 293 established plots along four transects, spanning from the valleys to the mountain tops, to represent the different types of forest structure in the national park (Bässler et al., 2008) (Fig 5.2). A stratified random sampling strategy based on aerial photo interpretation and previous forest inventory information was employed. First, based on tree species, the 293 plots were stratified into spruce dominated plots, beech dominated plots, and mixed plots. The mixed plots contained both spruce and beech, with also some other tree species like birch, fir, maple etc. Then, the plots were further stratified based on slope gradient and standard 


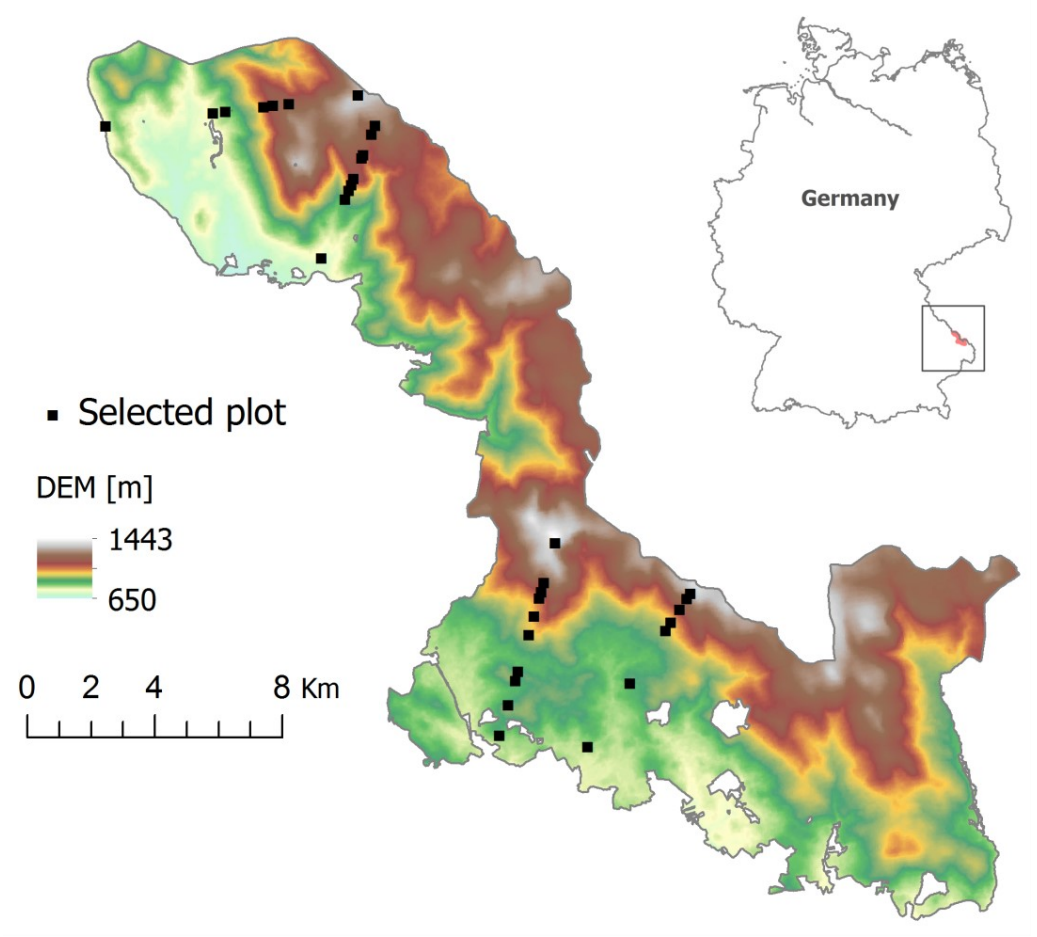

Figure 5.2: Study area (Bavarian Forest National Park) location in Germany and the location of the 33 selected plots

deviation of slope gradient $\left(0^{\circ}-3^{\circ}, 3^{\circ}-6^{\circ}, 6^{\circ}-9^{\circ}, \ldots\right)$. The 33 selected plots encompass a range of species composition, forest structures, and local topography conditions. Details of each plot are presented in Table 5.1. All subsequent analysis in this research was conducted in these 33 plots.

\subsection{Airborne LiDAR data}

From July 24 to 27,2012 , an airborne laser scanning campaign was conducted across the Bavarian Forest National Park by Milan Flug GmbH. The sensor on board was a Riegl LMS-Q680i laser scanner (wavelength $1550 \mathrm{~nm}$; pulse repetition frequency $350 \mathrm{KHz}$; nominal point density 30-40 points per $\mathrm{m}^{2}$; nominal footprint size $0.32 \mathrm{~m}$; flight height $650 \mathrm{~m}$ above ground). Both raw full-waveform data and point cloud data from Gaussian decomposition (Wagner et al., 2006) were delivered by Milan Flug. The discrete multiple return point cloud data was used for further analysis in this experiment. The point cloud composed of planimetric 
Table 5.1: Basic information of the 33 plots in the study area

\begin{tabular}{ccccl}
\hline Plot Name & Forest Type & Slope $\left[{ }^{\circ}\right]$ & Std of Slope $\left[^{\circ}\right]$ & $\begin{array}{l}\text { Elevation } \\
\text { Range }[\mathrm{m}]\end{array}$ \\
\hline T4-39 & Beech & 11.65 & 3.1 & 6.89 \\
T2-47 & Beech & 12.77 & 3.49 & 8.41 \\
T3-39 & Beech & 13.4 & 4.52 & 7.31 \\
T2-49 & Beech & 14.05 & 4.25 & 9.96 \\
T2-2 & Beech & 14.18 & 5.54 & 9 \\
T4-54 & Beech & 15.64 & 3.72 & 9.28 \\
T2-52 & Beech & 17.69 & 4.68 & 12.39 \\
T3-32 & Beech & 17.83 & 4.14 & 11.61 \\
T3-30 & Beech & 18.1 & 5.89 & 12.53 \\
T3-25 & Mix & 9.88 & 3.25 & 6.64 \\
T4-1 & Mix & 10.41 & 3.63 & 5.68 \\
T4-35 & Mix & 10.95 & 5.3 & 5.42 \\
T2-41 & Mix & 11.25 & 4.95 & 6.07 \\
T1-49 & Mix & 11.7 & 5.83 & 5.83 \\
T2-12 & Mix & 11.92 & 3.5 & 7.02 \\
T1-29 & Mix & 12.17 & 4.88 & 7.61 \\
T2-20 & Mix & 13.09 & 3.66 & 9.59 \\
T3-40 & Mix & 15.92 & 6.1 & 9.9 \\
T2-35 & Mix & 16.27 & 3.92 & 11.86 \\
T1-57 & Mix & 19.48 & 6.94 & 12.59 \\
T4-51 & Mix & 21.35 & 6.22 & 12.77 \\
T3-28 & Mix & 27.8 & 13.53 & 23.88 \\
T2-23 & Spruce & 6.74 & 3.49 & 3.78 \\
T3-5 & Spruce & 8.15 & 3.88 & 3.84 \\
T4-81 & Spruce & 11.45 & 3.94 & 8.03 \\
T1-5 & Spruce & 11.68 & 4.12 & 8.23 \\
T3-50 & Spruce & 11.92 & 6.42 & 7.22 \\
T1-52 & Spruce & 12.73 & 4.5 & 8.45 \\
T4-59 & Spruce & 12.99 & 3.66 & 8.51 \\
T3-47 & Spruce & 18.26 & 8.24 & 11.84 \\
T1-63 & Spruce & 18.67 & 6.06 & 13.61 \\
T1-61 & Spruce & 21.62 & 6.85 & 16.07 \\
T2-65 & Spruce & 27.59 & 7.21 & 20.51 \\
\hline Std of Slope: standard deviation of slope in the plot & & \\
\hline
\end{tabular}


coordinates ( $\mathrm{x}$ and $\mathrm{y}$ ) in the German local DHDN projection system (EPSG:31468), ellipsoidal heights (z), echo width, return intensity, return number, number of total returns for a laser shot, and GPS timestamp (of the return). In the preprocessing of LiDAR data, spurious isolated returns have been removed through noise filtering using the LAStools software (Isenburg, 2012).

\section{Method}

In this experiment, the vertical LAI profile and its derived metrics were calculated from both LiDAR point cloud data with and without topographic normalization. The results were then compared.

\subsection{Separation of ground and vegetation returns}

To calculate the vertical LAI profile, separation of ground returns versus non-ground (vegetation) returns is necessary. In this research, the separation of ground versus non-ground (vegetation) returns was carried out using the Cloth Simulation Filtering (CSF) algorithm (Zhang et al., 2016). This is a method integrated in the open source software Cloud Compare (Girardeau-Montaut, 2015) and hence accessible to any user (Zhang et al., 2016), which separates out the ground points by simulating a physical process where a virtual cloth drops down to an inverted (upside-down) point cloud. There are limited parameters in the CSF algorithm. In this study, the "relief scene" was selected, the cloth resolution was set to 0.5 , and the classification threshold was maintained at the default value of 0.5. In order to ensure classification accuracy, separation results were visually checked for each plot. Spurious points and errors were manually corrected by changing the classified label of the points.

\subsection{Topographic normalization}

All ground returns were used to calculate a Digital Terrain Model (DTM) using an inverse distance weighting interpolation method, one of the commonly used interpolation routines with a high accuracy (Su and Bork, 2006; Bater and Coops, 2009). The topographic surface elevation was 
then subtracted from all non-ground return heights. Thus, the relative height to the corresponding perpendicular ground location, instead of the ellipsoidal height to the sea level, was achieved.

$$
H_{\text {local }}=H_{\text {raw }}-E_{\text {ground }}
$$

where $H_{\text {local }}$ is the raw ellipsoidal height of the vegetation point to the sea level, $E_{\text {ground }}$ is the elevation height of the ground point with the same $\mathrm{x}, \mathrm{y}$ location as the vegetation point, $H_{\text {local }}$ is the resulting local height of the vegetation point.

\subsection{Calculation of vertical LAI profile}

The vertical LAI profile was then calculated based on the vertical $P_{g a p}$ profile of the canopy (Lovell et al., 2003; Coops et al., 2007). Owing to the inability to resolve the leaf angle distribution, clumping effect and woody materials effect, the vertical LAI profile derived here is more precisely referred to as an "apparent" vertical plant area index profile (Coops et al., 2007). Using discrete return LiDAR, the probability of a gap from the top of the canopy to a given height $h$, can be estimated through,

$$
P_{\text {gap }}(h)=1-\frac{N_{\text {vege }}(h)}{N_{\text {total }}}
$$

where $N_{\text {vege }}(h)$ is the number of laser pulses intercepted by vegetation elements down to a height $h$, and $N_{\text {total }}$ is the total number of emitted laser pulses (Lovell et al., 2003; Riaño et al., 2004; Coops et al., 2007). The cumulative LAI profile from the top of the canopy down to a height $h$ is then given by,

$$
L(h)=-\ln \left(P_{\text {gap }}(h)\right)
$$

where the first derivative of $L(h)$ is the vertical LAI profile. In this study, the vertical LAI profile was calculated using $0.5 \mathrm{~m}$ height bins (i.e., $0.5 \mathrm{~m}$ height intervals in the vertical LAI profile). Other bin sizes, such as $1 \mathrm{~m}$ or $2 \mathrm{~m}$, could also be used, leading to LAI profiles of a different vertical resolution. 


\subsection{Metrics derived from the vertical LAI profile}

To compare the results of vertical LAI profiles, three metrics derived from the vertical LAI profiles were also calculated and compared.

\section{* Vertical Extent}

$$
V E=H_{\max }-H_{\min }
$$

where $H_{\max }$ is the maximum height of the vertical LAI profile, and $H_{\min }$ is the minimum height of the vertical LAI profile. This metric describes the vertical extent of the foliage distribution. The higher this metric is, the wider and more spreading the leaf area vertical distribution is. The smaller this metric is, the more vertically condensed the leaf area distribution is.

\section{* Canopy Layer Number}

Canopy layer number is a categorical metric derived from the vertical LAI profile and can be used to quantify the complexity of the vertical forest structure. The higher the canopy layer number is, the more complex the vertical LAI profile and vertical forest structure is. Canopy layer has been calculated in other research using either a pre-defined height threshold (Whitehurst et al., 2013; Latifi et al., 2015) or automatic determination (Leiterer et al., 2015; Wilkes et al., 2015a). The pre-defined height threshold method limits the determination of canopy layers to a maximum of three layers (i.e., under-story, middle-story, and upper-story). In order to illustrate the subtle differences between plots of multiple $(4,5$ or more) layers, we therefore followed the automatic method (Wilkes et al., 2015a). Firstly, the vertical LAI profile was smoothed to remove signal noise. Secondly, the local maximum location of the vertical LAI profile was calculated to be the location of each canopy layer. And thirdly, layers with a leaf area of less than $5 \%$ of the maximum leaf area layer were removed. Neighboring layers with a height distance of less than 2 meters and leaf area density differences of less than $5 \%$ were merged into one layer.

In the smoothing of the vertical LAI profile, Gaussian smoothing $(\sigma=0.8)$ was used instead of the nonparametric cubic spline smoothing (Muss 
et al., 2011; Wilkes et al., 2015a). Gaussian smoothing was chosen because the results of our experiment showed that it could best preserve the differences between vertical LAI profiles of point clouds with and without topographic normalization. Nonparametric cubic spline smoothing, on the other hand, severely over-smoothed the two profiles and eliminated their differences.

\section{* Entropy}

In addition to the canopy layer number, the entropy of the vertical LAI profile was also calculated. In information theory, Shannon's entropy is a measure of uncertainty and information formulated in terms of probability theory (Rrnyi, 1961). In geoscience, it has been used to measure the degree of spatial concentration or dispersion of a geophysical variable among n spatial units/zones (wards) (Foody, 1996; Jat et al., 2008). Unlike the categorical metric "canopy layer number", the entropy is a continuous index describing the vertical complexity of leaf area distribution. Compared to the canopy layer number, the entropy metric is more generally applicable and comparable across different research areas and different datasets, as well as less subjective to definitions. Similar as for the canopy layer number calculation, Gaussian smoothing ( $\sigma=0.8$ ) was applied to the raw vertical LAI profile prior to entropy calculation, to reduce noise. Then, entropy was calculated through

$$
\text { Entropy }=\sum_{i}-p_{i} \ln p_{i}
$$

where $p_{i}$ is the proportion of leaf area in height bin $i$. The entropy value increases with the vertical extent of the LAI profile and also with a more equal distribution of leaf area density across the profile (Stark et al., 2012). Large entropy values indicate a more continuous and complex distribution of the vertical LAI profile in the plot and potentially a more complex vertical structure as well as more niche space available to support diverse species in the plot.

\subsection{Statistical analysis}

To evaluate whether topographic normalization would change the vertical LAI profile and its complexity, three two-sample $t$-tests were performed using the results of the vertical extent, the canopy layer number, and 
the entropy of the vertical LAI profile, respectively. The null hypothesis is that there is no statistically significant difference between the abovementioned three metrics derived from either the topographic normalized point cloud or the original point cloud data.

In addition, in order to explore how local topography affects the vertical LAI profile after topographic normalization, linear regression was conducted between the degree of vertical LAI profile change (i.e., vertical extent change, canopy layer number change, and entropy change), and parameters (i.e., mean slope and standard deviation of slope) representing local topography. Mean slope was utilized to represent the steepness of the local topography. Standard deviation of slope was employed to describe how rough the local topography was. This is an effective measure of surface roughness, as it is simple to calculate, detects fine scale/regional relief, and performs at a variety of scales (Grohmann et al., 2011).

\section{Results}

\subsection{Visual comparison}

Examples of vertical LAI profiles for six plots with different local topography (increasing average slope and standard deviation of slope) are shown in Fig 5.3. There are many differences between the two vertical LAI profiles with and without topographic normalization, respectively. Firstly, the vertical extent of the LAI distribution is different. Before topographic normalization, the LAI profile has a wider vertical extent and a more continuous distribution. After normalization, the LAI profile has a shorter vertical extent and appears to be condensed. Secondly, the location and value of maximum leaf area density are also different. After topographic normalization, the maximum leaf area density becomes much higher than before (plots T2-23 and T2-65). Altogether, with increasing slope and topography roughness (standard deviation of slope), the vertical condensing effect is augmented.

In addition, regarding the curve shape of each vertical LAI profile, there are differences (i.e., plots T2-23, T2-49, T4-51, and T2-65), with vertical 
LAI profiles having a very different trend, number of modes, and location of modes. Generally, after normalization, the mode number is reduced.
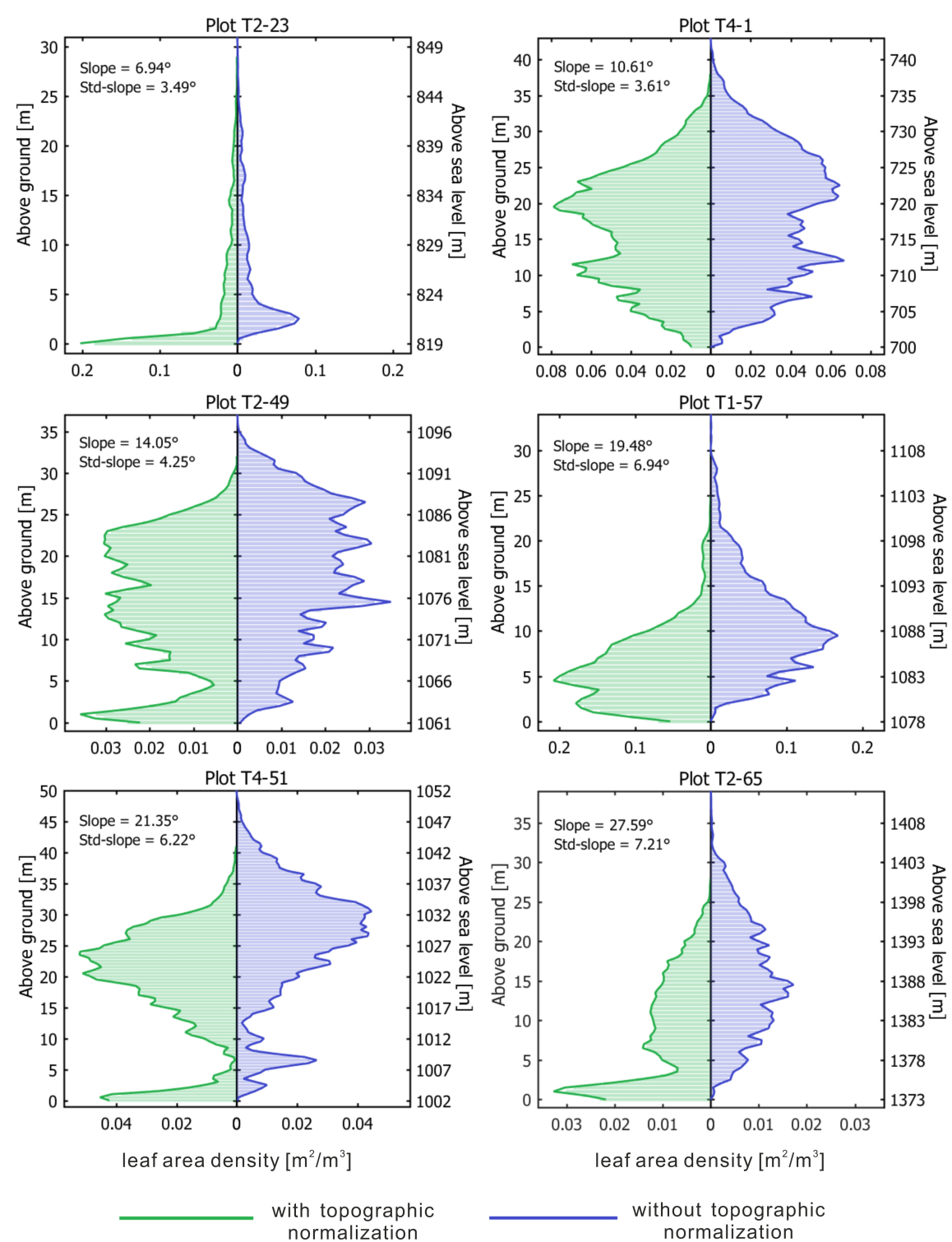

Figure 5.3: Comparison between the vertical LAI profiles with and without topographic normalization (bar: raw LAI profile; curve: smoothed LAI profile). 
An example (plot T3-28) of the canopy layer number determination from the vertical LAI profile is shown in Fig 5.4. Before topographic normalization, six layers are detected. Except for the upper two layers, the lower four layers have a high leaf area concentration and large gaps between them. After topographic normalization, only three layers are detected. And a large gap can only be discerned between the bottom layer and the upper layers.
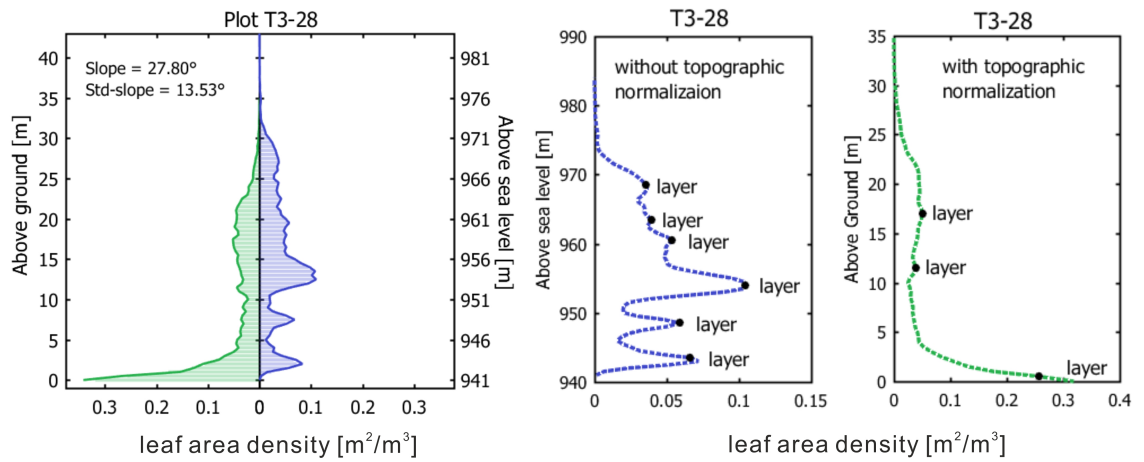

Figure 5.4: Comparison between canopy layers detected from vertical LAI profiles with (in green) and without (in blue) topographic normalization

\subsection{Quantitative comparison}

The results of derived metrics from the vertical LAI profiles of each plot are detailed in Table 5.2. After topographic normalization, the vertical extent of the LAI profile decreases for all plots. The extent of this decrease ranges from $2 \mathrm{~m}$ to $11 \mathrm{~m}$. Since the plot size is only $30 \mathrm{~m}$ by 30 $\mathrm{m}$, this decrease is quite significant. This shows that after topographic normalization, the LAI profile is vertically condensed.

The other two metrics, entropy and canopy layer number, describe the complexity of the vertical LAI profile and thus the complexity of the forest vertical structure of each plot. For the continuous metric "entropy", 31 out of 33 plots have a decreased entropy value after topographic normalization. For the categorical metric "canopy layer number", out of the 33 plots, 2 plots have more layers, 7 plots have the same number of layers, while 24 plots have less layers after topographic normalization. Analyzing the average of all plots, before topographic normalization, the averaged entropy is 3.81 , while the average number of detected layers is 
5.59. However, after topographic normalization, the averaged entropy value decreases to 3.49 , and average number of detected layers decreases to 4.09 .

Table 5.2: The vertical complexity of canopy structure derived from the vertical LAI profiles with (TN) and without topographic normalization (noTN) and their differences (diff)

\begin{tabular}{|c|c|c|c|c|c|c|c|c|c|c|}
\hline \multirow{2}{*}{$\begin{array}{l}\text { Plot } \\
\text { Name }\end{array}$} & \multirow{2}{*}{$\begin{array}{l}\text { Forest } \\
\text { Type }\end{array}$} & \multicolumn{3}{|c|}{ Vertical Extent [m] } & \multicolumn{3}{|c|}{ Canopy Layer Number } & \multicolumn{3}{|c|}{ Entropy } \\
\hline & & notN & $\mathrm{TN}$ & diff & notN & $\mathrm{TN}$ & diff & notN & $\mathrm{TN}$ & diff \\
\hline T4-39 & Beech & 47 & 44 & 3 & 9 & 7 & 2 & 4.3 & 4.3 & 0 \\
\hline $\mathrm{T} 2-47$ & Beech & 39 & 33 & 6 & 6 & 5 & 1 & 3.87 & 3.63 & 0.24 \\
\hline T3-39 & Beech & 37 & 31 & 6 & 3 & 3 & 0 & 3.77 & 3.62 & 0.15 \\
\hline $\mathrm{T} 2-49$ & Beech & 37 & 32 & 5 & 8 & 5 & 3 & 4.11 & 3.92 & 0.19 \\
\hline $\mathrm{T} 2-2$ & Beech & 41 & 36 & 5 & 5 & 4 & 1 & 3.81 & 3.63 & 0.18 \\
\hline T4-54 & Beech & 35 & 27 & 8 & 4 & 5 & -1 & 3.72 & 3.55 & 0.17 \\
\hline $\mathrm{T} 2-52$ & Beech & 39 & 28 & 11 & 7 & 3 & 4 & 3.99 & 3.6 & 0.39 \\
\hline T3-32 & Beech & 40 & 34 & 6 & 5 & 5 & 0 & 4.09 & 3.92 & 0.17 \\
\hline T3-30 & Beech & 41 & 33 & 8 & 6 & 4 & 2 & 3.83 & 3.73 & 0.1 \\
\hline T3-25 & Mix & 50 & 45 & 5 & 7 & 6 & 1 & 4.18 & 4.16 & 0.02 \\
\hline T4-1 & Mix & 43 & 38 & 5 & 6 & 5 & 1 & 4.18 & 4.07 & 0.11 \\
\hline T4-35 & Mix & 45 & 42 & 3 & 10 & 7 & 3 & 4.11 & 4.07 & 0.04 \\
\hline $\mathrm{T} 2-41$ & Mix & 39 & 35 & 4 & 7 & 6 & 1 & 4.05 & 3.85 & 0.2 \\
\hline T1-49 & Mix & 32 & 30 & 2 & 5 & 5 & 0 & 3.88 & 3.76 & 0.12 \\
\hline $\mathrm{T} 2-12$ & Mix & 33 & 27 & 6 & 5 & 5 & 0 & 3.83 & 3.71 & 0.12 \\
\hline T1-29 & Mix & 35 & 30 & 5 & 5 & 4 & 1 & 3.72 & 3.53 & 0.19 \\
\hline $\mathrm{T} 2-20$ & Mix & 39 & 36 & 3 & 5 & 2 & 3 & 3.64 & 3.57 & 0.07 \\
\hline T3-40 & Mix & 37 & 35 & 2 & 6 & 5 & 1 & 3.86 & 3.83 & 0.03 \\
\hline $\mathrm{T} 2-35$ & Mix & 46 & 41 & 5 & 8 & 6 & 2 & 4.22 & 4.18 & 0.04 \\
\hline $\mathrm{T} 1-57$ & Mix & 34 & 25 & 9 & 6 & 3 & 3 & 3.68 & 3.35 & 0.33 \\
\hline T4-51 & Mix & 50 & 41 & 9 & 10 & 7 & 3 & 4.24 & 3.93 & 0.31 \\
\hline T3-28 & Mix & 43 & 35 & 8 & 6 & 3 & 3 & 4.02 & 3.7 & 0.32 \\
\hline $\mathrm{T} 2-23$ & Spruce & 31 & 29 & 2 & 3 & 1 & 2 & 3.06 & 2.4 & 0.66 \\
\hline Т3-5 & Spruce & 14 & 12 & 2 & 1 & 1 & 0 & 2.98 & 2.88 & 0.1 \\
\hline T4-81 & Spruce & 15 & 12 & 3 & 1 & 1 & 0 & 3.01 & 1.86 & 1.15 \\
\hline $\mathrm{T} 1-5$ & Spruce & 43 & 40 & 3 & 6 & 7 & -1 & 4.18 & 4.18 & 0 \\
\hline T3-50 & Spruce & 9 & 7 & 2 & 2 & 1 & 1 & 2.52 & 1.76 & 0.76 \\
\hline T1-52 & Spruce & 40 & 33 & 7 & 8 & 3 & 5 & 3.77 & 2.66 & 1.11 \\
\hline T4-59 & Spruce & 40 & 37 & 3 & 6 & 4 & 2 & 4.15 & 3.87 & 0.28 \\
\hline T3-47 & Spruce & 40 & 29 & 11 & 8 & 7 & 1 & 4.02 & 3.6 & 0.42 \\
\hline T1-63 & Spruce & 31 & 22 & 9 & 2 & 2 & 0 & 3.36 & 2.8 & 0.56 \\
\hline T1-61 & Spruce & 34 & 24 & 10 & 6 & 2 & 4 & 3.94 & 3.02 & 0.92 \\
\hline T2-65 & Spruce & 39 & 28 & 11 & 6 & 4 & 2 & 3.98 & 3.45 & 0.53 \\
\hline
\end{tabular}

The $t$ value of the three two sample $t$-tests is shown in Table 5.3. In this study, with 64 degrees of freedom (33 plots), a $t$ value greater than 1.669 or lower than -1.669 allows us to reject the null hypothesis at a 95\% confidence level. For all three metrics (i.e., the vertical extent, canopy layer number, and the entropy of vertical LAI profiles), the $t$ values are greater than 1.669. Therefore, all three metrics are significantly higher, 
statistically, before topographic normalization than after topographic normalization.

Table 5.3: Summary of the two sample $t$-test statistics for canopy vertical complexity metrics with and without topographic normalization

\begin{tabular}{lc}
\hline & $t$ value \\
\hline vertical extent & 2.51 \\
canopy layer number & 2.88 \\
entropy & 2.28 \\
\hline
\end{tabular}

The statistical results between the change of vertical LAI profile complexity and local topography condition are shown in Fig 5.5. The mean slope is found to have a stronger statistical relationship with the decrease of vertical extent of LAI profile, than the standard deviation of slope (topography roughness). With increasing mean slope, topographic normalization causes a greater decrease in the vertical extent.
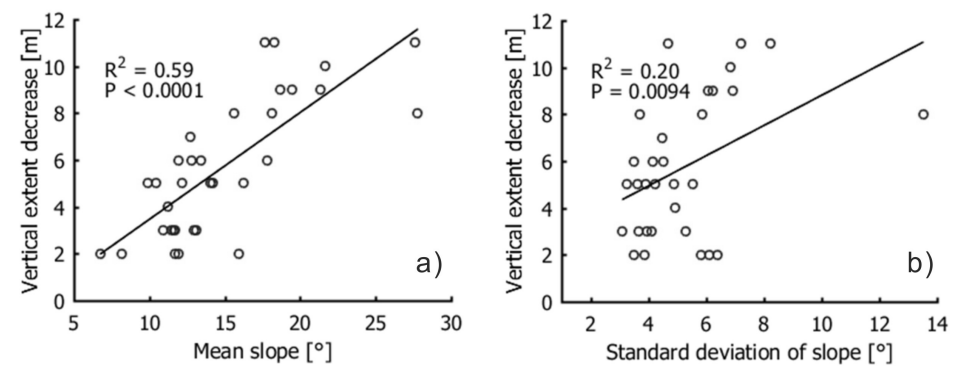

Figure 5.5: (a) Relationship between the decrease of vertical extent and mean slope; (b) Relationship between the decrease of vertical extent and standard deviation of slope

For most plots, the canopy layer number decreases after topographic normalization (Fig 5.6). However, while the canopy layer number decreases, there is no significant statistical correlation between the decrease in canopy layer number and either the mean slope (Fig 5.6 a)) or the standard deviation of slope (Fig $5.6 \mathrm{~b}$ )). Similar patterns are observed for the entropy of the vertical LAI profile. For almost all plots, the entropy decreases after topographic normalization. However, there is no significant statistical correlation between the degree of entropy decrease and either mean slope (Fig 5.6 c)) or standard deviation of slope (Fig 5.6 d)). 

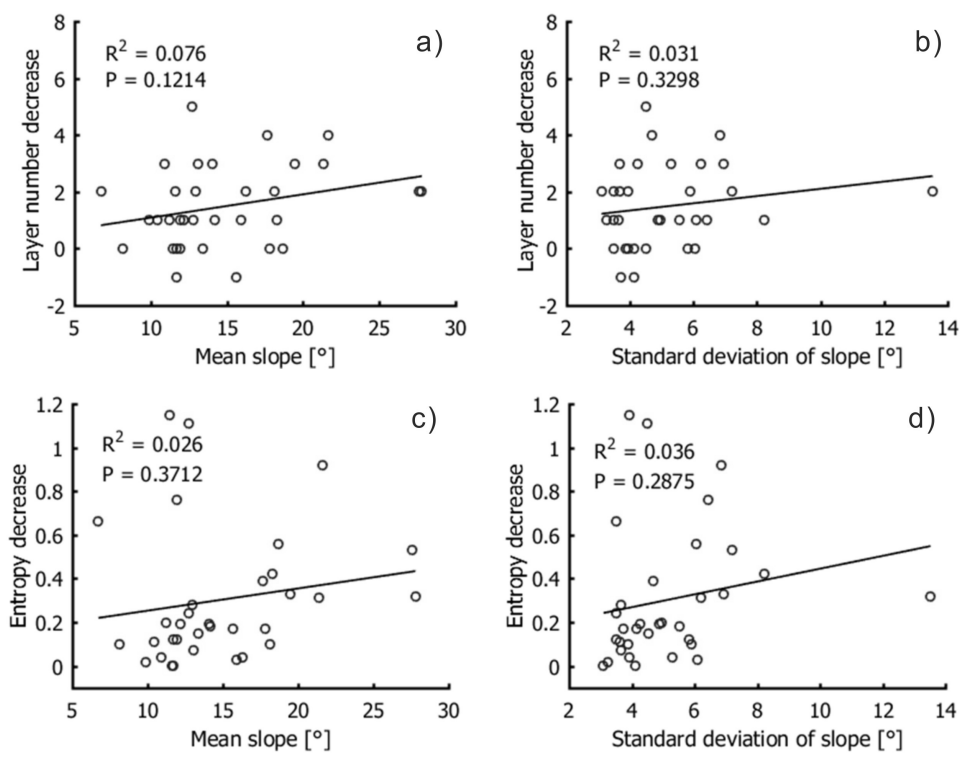

Figure 5.6: Relationship between a) canopy layer number decrease and mean slope, b)canopy layer number decrease and standard deviation of slope, c) entropy decrease and mean slope, d) entropy decrease and standard deviation of slope

\section{Discussion and conclusion}

Our study shows that topographic normalization significantly changes the vertical extent and curve shape of a vertical LAI profile. After topographic normalization, the spatial distribution of the original LiDAR point cloud will result in all trees being relocated on a flat plane. Although the height of each individual tree maybe maintained, the spatial arrangement changes. If the local topography is not flat, trees that are "downhill" or in the "basin" will "rise" in the corrected plot, while trees originally "uphill" or at the "summit" will "sink", comparatively, in the new plot (Khosravipour et al., 2015). This effect can be clearly seen in Fig 5.7, where the original LiDAR point cloud and topographic normalized point cloud are both depicted. Previous research has found that both tree crown shape and tree top location may be systematically distorted in this process of topographic normalization (Khosravipour et al., 2015). This research proves that the vertical LAI profile will also be distorted and vertically condensed, and that the maximum leaf area density will 
be increased.

a) without topographic normalization

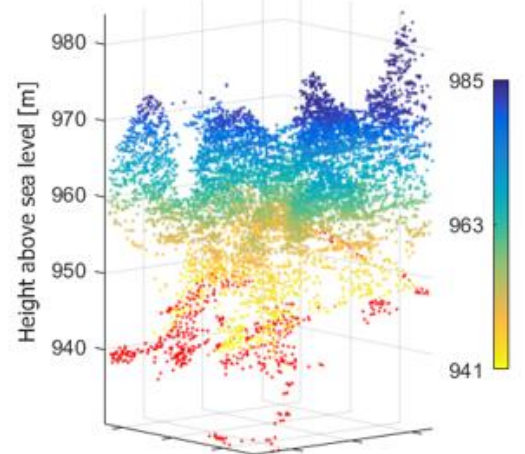

b) with topographic normalization

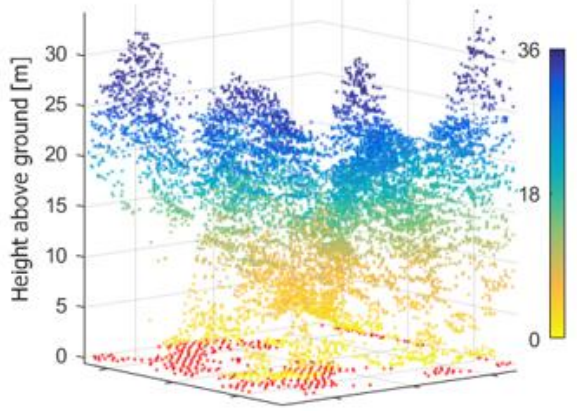

Figure 5.7: Comparison between point clouds of plot T3-28 with and without topographic normalization, respectively. (Same viewing angle and azimuth angle, red points are detected ground returns)

The results of the two-sample $t$-tests prove that after topographic normalization, the complexity of vertical LAI profiles (described by canopy layer number and entropy) also decreases. This means that topographic normalization reduces the complexity of the original forest vertical structure. However, the degree of change in structural complexity is not linearly related to topographic variables such as mean slope and standard deviation of slope. This indicates that, as the vertical LAI profile's complexity decreases, the degree of change is not solely determined by the steepness and roughness of the local topography. Contrary to common expectation, more complex topography does not necessarily lead to more change. Fig 5.8 shows a theoretical case, where two plots have exactly the same local topography and the same vegetation. However, how the plants are spatially distributed across the plots is different. This leads to a different vertical structure, different vertical LAI profiles, and different canopy layers. But after topographic normalization, the new vertical LAI profiles are the same. As a result, the decrease in the vertical LAI profiles' complexity differs. This example clearly shows that the change of the complexity of vertical LAI profile can be different even when the local topography is the same.

Therefore, it can be concluded that, the local topographic condition adds to the complexity of the forest vertical structure. In other words, 
topographic normalization can undermine the complexity of the vertical LAI profiles. However, the degree of this change, is not a linear function of the mean slope or the standard deviation of slope in the local topography, but is determined by the interaction of local topography and how the trees are distributed across the landscape surface. There are other terrain attributes such as slope aspect, profile curvature, and planform curvature which can also affect the forest vertical structure. But since the effect of topographic normalization on vertical LAI profiles is not dependent solely on topography or solely on tree distribution (Fig 5.8 ), no further quantitative analysis is conducted in this experiment.

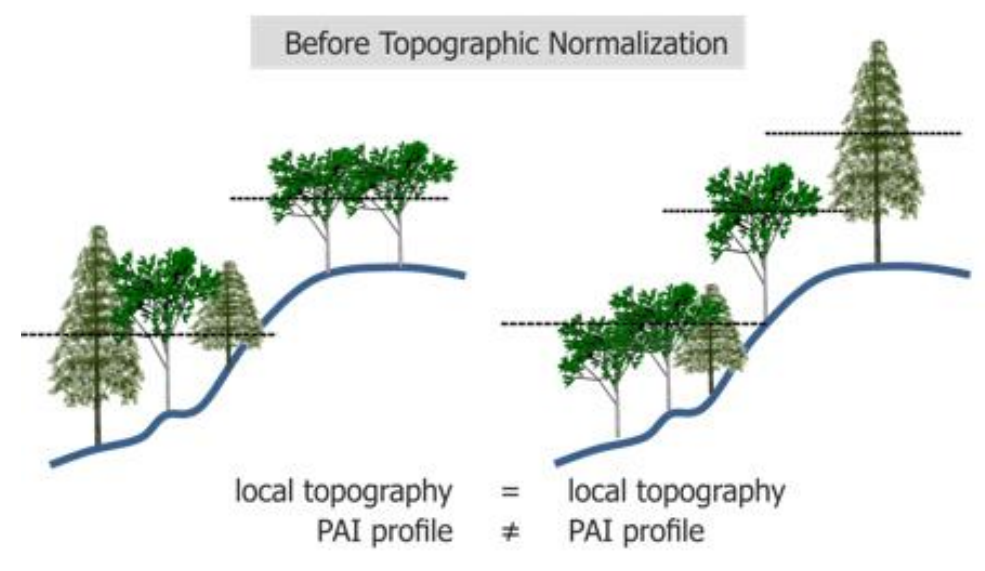

After Topographic Normalization

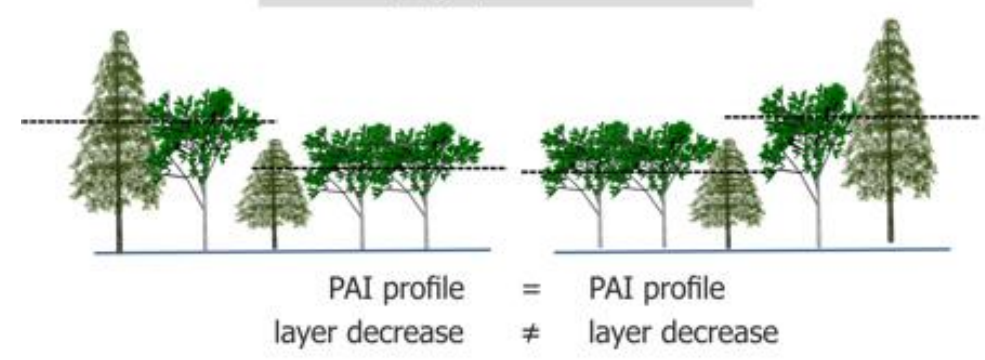

Figure 5.8: Illustration of different decrease of vertical LAI/PAI profile complexity after topographic normalization, even with the same local topography and same vegetation. The differences are caused by the different spatial distribution of trees. (dashed line: estimated canopy layer location)

The results of this research provide us with a better understanding of the retrieval of vertical LAI profiles from airborne LiDAR data, which either 
have, or have not, been subjected to topographic normalization. The vertical LAI profile derived from airborne LiDAR point cloud without topographic normalization can inform us about the actual height of canopy with the maximum leaf area concentration, the minimum and maximum height of plants, as well as the number of layers and the complexity of the vertical structure. All these information is ecologically important and useful, especially for plot radiation regime analysis (Parker et al., 2001) and species distribution modeling. For example, in mature forests, bat activity is driven by vegetation density and presence of gaps (Muller et al., 2013). A vertical LAI profile distorted by topographic normalization will give a wrong estimate of vegetation density and gap presence in space, and probably lead to errors in bat species distribution modeling. Also, the vertical LAI profile without topographic normalization may serve as a more accurate input parameter for estimating stand canopy bulk density and forest fire modeling, because it preserves the spatial connectivity between forest fuels. This allows more accurate modelling of fire propagation, as well as assessment of fire-atmosphere interactions (Contreras et al., 2012). The vertical LAI profile without topographic normalization may also serve as a more accurate input for forest precipitation infiltration modeling, because it can preserve the original spatial topology between vegetation layers, while topographic normalization creates distortion and offsets in the vertical distribution of plants.

The vertical LAI profile derived from airborne LiDAR data with topographic normalization can change the forest vertical structure from an ecological perspective. The height of maximum leaf area concentration, canopy layer number and canopy layer location cannot be correctly estimated from a topographically corrected LAI profile. However, some other information, such as the maximum tree height in a plot (Lovell et al., 2003; Calders et al., 2014), canopy height quantile metrics, and above ground biomass can still be directly retrieved with reasonable accuracy. The reason is that topographic normalization mainly alters the relative distribution among vegetation, not the relative distribution of vegetation to the ground. This was also demonstrated in previous research, where on a slope less than $15^{\circ}$, there is no significant canopy height displacement error after topographic normalization (Khosravipour et al., 2015). Another possible application of vertical LAI profiles with 
topographic normalization is the modelling of soil erosion. In soil splash erosion, the relative distance between the substrate and the vegetation layer determines the likelihood of erosion (Nanko et al., 2008; Geißler et al., 2012). Then vertical LAI profiles with topographic normalization are preferred in this case, because they contain the relative location of vegetation to ground, which is more important than the relative location among different vegetation layers.

From this research, it is recommended whether topographic normalization is applied or not, depends on for which application the vertical LAI profile is further used. For ecological modelling, topographic normalization should not be applied, when the ecological process is largely determined by the three dimensional structure and the spatial topology of vegetation inside the plot. Because slope can create distortion and vertical offset in the vertical LAI profiles. These applications include radiation regime analysis, species distribution modeling, and fire propagation modelling. However, for forest inventory, including canopy height and above ground biomass estimation, it is recommended that the topographic normalization effect can be ignored below a certain threshold of slope degree. Because in these applications, the accuracy of height or crown diameter of individual tree is far more important than the spatial topology of neighboring trees. Due to the limited plot number in our study, and the relatively small range of slope degree in the study area, further analysis in more complex topography areas is necessary to recommend a definitive threshold, currently a figure around $15^{\circ}$ seems reasonable based the results of this study and the previous published material (Khosravipour et al., 2015). 
Synthesis: Mapping the 3D distribution of leaves in forests using LiDAR 


\section{Summary}

Leaf area index (LAI) is a primary descriptor of vegetation structure. It has been identified as an essential climate variable (Baret et al., 2013) and an essential biodiversity variable (Skidmore and Pettorelli, 2015). The vertical LAI profile is a more detailed description of the 3D leaf area distribution inside the canopy. Due to the limitations of passive optical sensors such as signal saturation and inability to resolve the vertical distribution, LiDAR plays an indispensable role in mapping the 3D distribution of leaves in vegetation canopies. Nevertheless, accurate 3D mapping of leaves is a challenging task for forests, owing to the complexity of canopy structure, underlying topography, and LiDAR settings.

This thesis evaluated several key factors in the LAI and vertical LAI profile retrieval using LiDAR data at local and regional scale. These factors include the leaf angle distribution (LAD), gap fraction $\left(P_{\text {gap }}\right)$, LiDAR scan angle $(\theta)$, and uneven topography, all of which were parameters in the physically based gap fraction model to estimate LAI and vertical LAI profile. Chapter 2 examined which in-situ technique produced more accurate LAD estimate. Using field-based and simulation dataset, terrestrial LiDAR was proved more accurate than DHP when estimating LAD in broadleaf forests. Chapter 3 examined whether the spherical LAD assumption was valid for natural European beech forests. Using terrestrial LiDAR, large $\mathrm{LAD}$ variation was demonstrated both in different stands and in different canopy layers. A uniform distribution rather than a spherical distribution was a more valid LAD assumption. Chapter 4 evaluated the effect of airborne LiDAR flight settings, in particular the scan angle, on the retrieval of $P_{\text {gap }}$ and vertical $P_{\text {gap }}$ profile. The results proved the underestimation of $P_{\text {gap }}$ amplified at large off-nadir scan angle. It implied that large off-nadir scan angle LiDAR data should be avoided to ensure a more accurate $P_{\text {gap }}$ and LAI estimation. Chapter 5 assessed the effect of uneven topography and topographic normalization in the vertical LAI profile retrieval. The findings demonstrated that topographic normalization undermined the complexity of the vertical LAI profile. For ecological applications, such as biodiversity modeling, topographic normalization was suggested not to be applied. 


\section{Retrieving LAI and vertical LAI profile at local scale}

\subsection{Urgency to identify the optimal in-situ LAI technique}

A crucial step in LAI mapping is the collection of in-situ LAI reference. Accurate LAI measurement at local scale is of crucial importance for calibrating and validating regional or global products. Existing LAI products from passive optical sensors, for instance from MODIS, SPOT, and MERIS, heavily rely on indirect methods (such as LAI-2000 and DHP) to collect LAI ground reference.

Even though LAI from these indirect methods were considered as the "true" reference, inconsistency in in-situ measurements were revealed among different indirect methods (Garrigues et al., 2008). The uncertainty and inconsistency is especially high in forests (Woodgate et al., 2015; Calders et al., 2018). For example, LAI from terrestrial LiDAR was found to be on average 55\% higher than LAI from DHP in Eucalypt forests (Woodgate et al., 2015). This is much higher than the targeted $5 \%$ uncertainty levels stipulated by the World Meteorological Organization. Therefore, it is urgent to ascertain which in-situ indirect technique measures LAI the most reliably and accurately.

\subsection{Synthetic dataset to validate and benchmark in-situ techinques}

Because destructive sampling is very costly and sometimes impractical, generating a synthetic dataset by computer simulation offers an alternative to validate and identify the most accurate in-situ LAI method. Chapter 2 demonstrated a good example. In order to compare either terrestrial LiDAR or DHP measures LAD more accurately, the true inclination angle of each leaf is required. This almost impossible task in real world forests, can be easily achieved through computer simulation. The shape, area, location and inclination of each leaf is known during the virtual forest creation. As a result, a synthetic dataset enables comparison of the absolute LAI accuracy from different in-situ techniques. Chapter 2 proved terrestrial LiDAR gave much more accurate estimate of LAD than DHP in broadleaf forests $\left(R^{2}: 0.79>0.12\right.$, RMSE: $\left.6.41^{\circ}<16.40^{\circ}\right)$, as seen in Fig 6.1. 

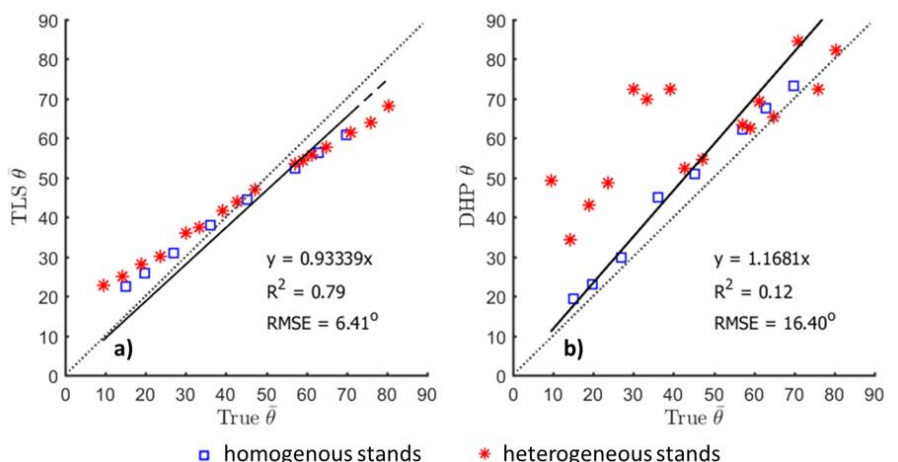

Figure 6.1: a) terrestrial LiDAR (TLS) estimated the average inclination angle $\bar{\theta}$ much more accurately than $\mathrm{b}$ ) digital hemispherical photography (DHP) in the synthetic dataset

Terrestrial LiDAR holds great potential in acquiring in-situ LAI reference, as well as validating products from passive optical sensors. Chapter 2 and Chapter 3 in this thesis showed that terrestrial LiDAR yielded a much more accurate estimate of LAD compared to the conventional device DHP, both at individual leaf level and at stand level. Previous studies demonstrated the superiority of terrestrial LiDAR compared to DHP or LAI-2000 in measuring gap fraction $\left(P_{\text {gap }}\right)$, due to its active feature and insensitivity to illumination conditions (Calders et al., 2018). Furthermore, the differentiation of woody elements and foliage, which used to be quite challenging in DHP or LAI-2000, has been accomplished with high accuracy from terrestrial LiDAR (Li et al., 2018b; Zhu et al., 2018a; Ferrara et al., 2018). Altogether, more accurate estimate of $P_{\text {gap }}$, LAD and woody elements, in theory, will result in much more accurate LAI estimate using terrestrial LiDAR than DHP or LAI-2000. Further research will be explored to validate this inference.

\section{Retrieving LAI and vertical LAI profile at regional scale}

\subsection{Superiority of airborne LiDAR in LAI mapping in dense forest}

Using the method described in section 3.1 of Chapter 1 , the regional LAI map from the Bavarian Forest National Park in the year 2017 is 
retrieved in Fig 6.2. While LAI from passive optical sensors saturate at dense forest when LAI is higher than 3 to 4 (Asner et al., 2003; Liu et al., 2018b), LAI retrieved from airborne LiDAR can be as large as 9.2. This is a range exceeding current observation limits by passive sensors (Tang, 2015). It demonstrates the superiority of airborne LiDAR to map LAI in forests of dense vegetation. The results are consistent with previous studies in forests of medium to high biomass in previous studies (Jensen et al., 2008). Similarly, Vincent et al. (2017) proved fullwaveform airborne LiDAR can produce accurate estimates even when the LAI value is reaching 10 or 13 in tropical forests.

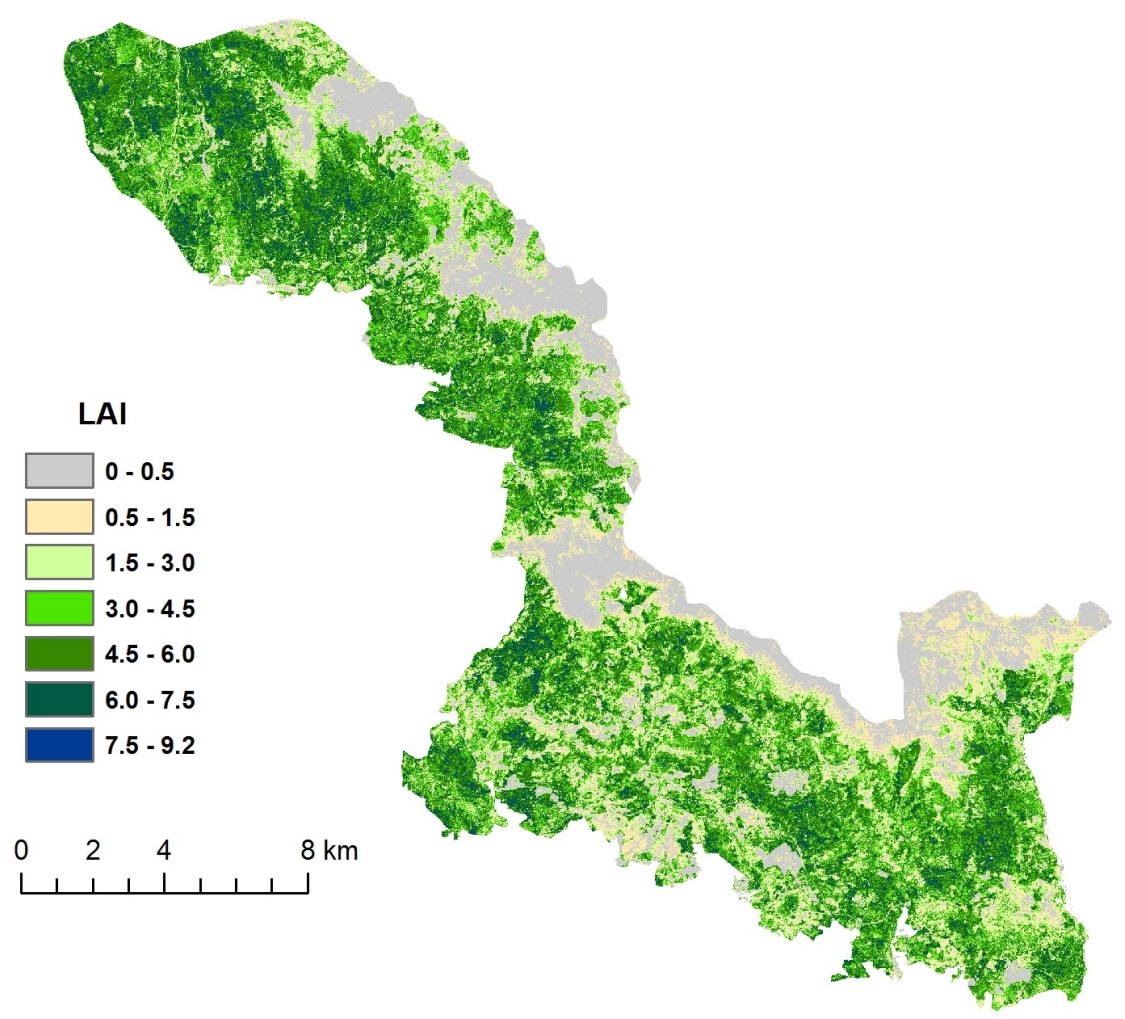

Figure 6.2: The LAI map retrieved from airborne LiDAR data in the Bavarian Forest National Park in 2017

Furthermore, the LAI estimated from airborne LiDAR was compared to the LAI estimated from DHP (Fig 6.3). From the results, there is moderate consistency of LAI estimated from airborne LiDAR and DHP $\left(R^{2}=0.53\right.$, RMSE $=1.12$ ). Since there is high uncertainty in the LAI estimated from 
DHP techniques in forests (Woodgate et al., 2015; Calders et al., 2018), further results evaluation should be conducted using leaf collection measurements.

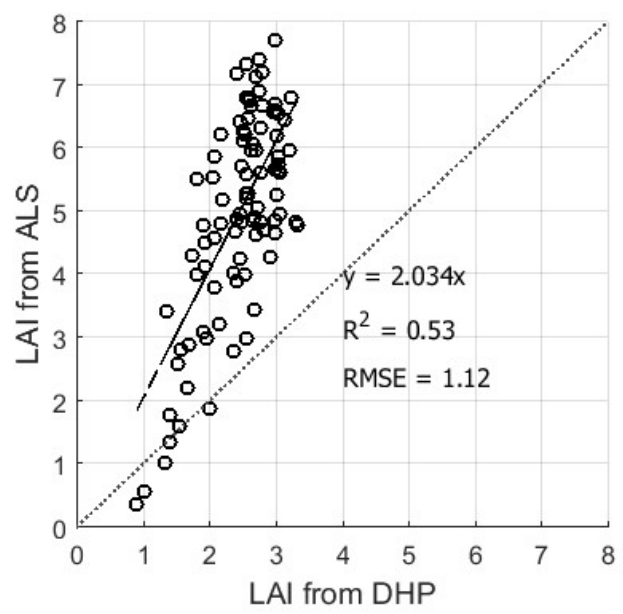

Figure 6.3: Consistency of LAI estimated from airborne LiDAR and DHP

\subsection{Irreplaceable role of airborne LiDAR in vertical LAI profile mapping}

Airborne LiDAR plays a unique and irreplaceable role in mapping the vertical LAI profile of forests. For easier visualization, the vertical LAI profile was displayed in RGB composite of LAI in three layers (top layer, middle layer and bottom layer) as shown in Fig 6.4. Compared to the LAI map in Fig 6.2, the vertical LAI profile map presents not only the horizontal distribution, but also the vertical distribution of leaves in forests. For instance, the area 1 and area 2 have similar LAI ranges and horizontal LAI heterogeneity, as seen in Fig 6.5 a) and c). However, they displayed distinct vertical structure in the vertical LAI profile map, as seen in Fig 6.5 b) and d). Airborne LiDAR plays an irreplaceable role in mapping the fine resolution vegetation 3D distribution in forests. 


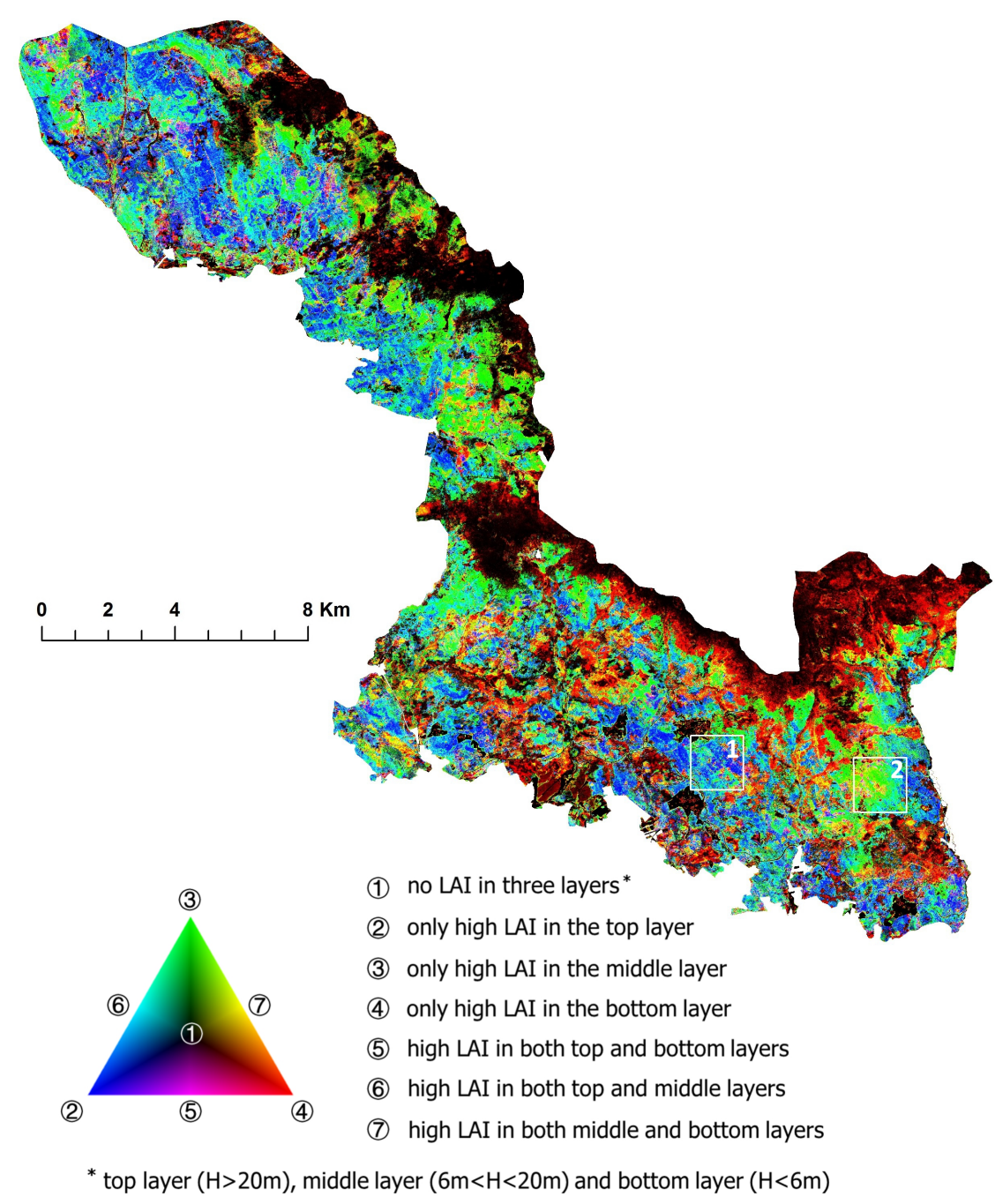

Figure 6.4: The vertical LAI profile map retrieved from airborne LiDAR in the Bavarian Forest National Park in 2017 

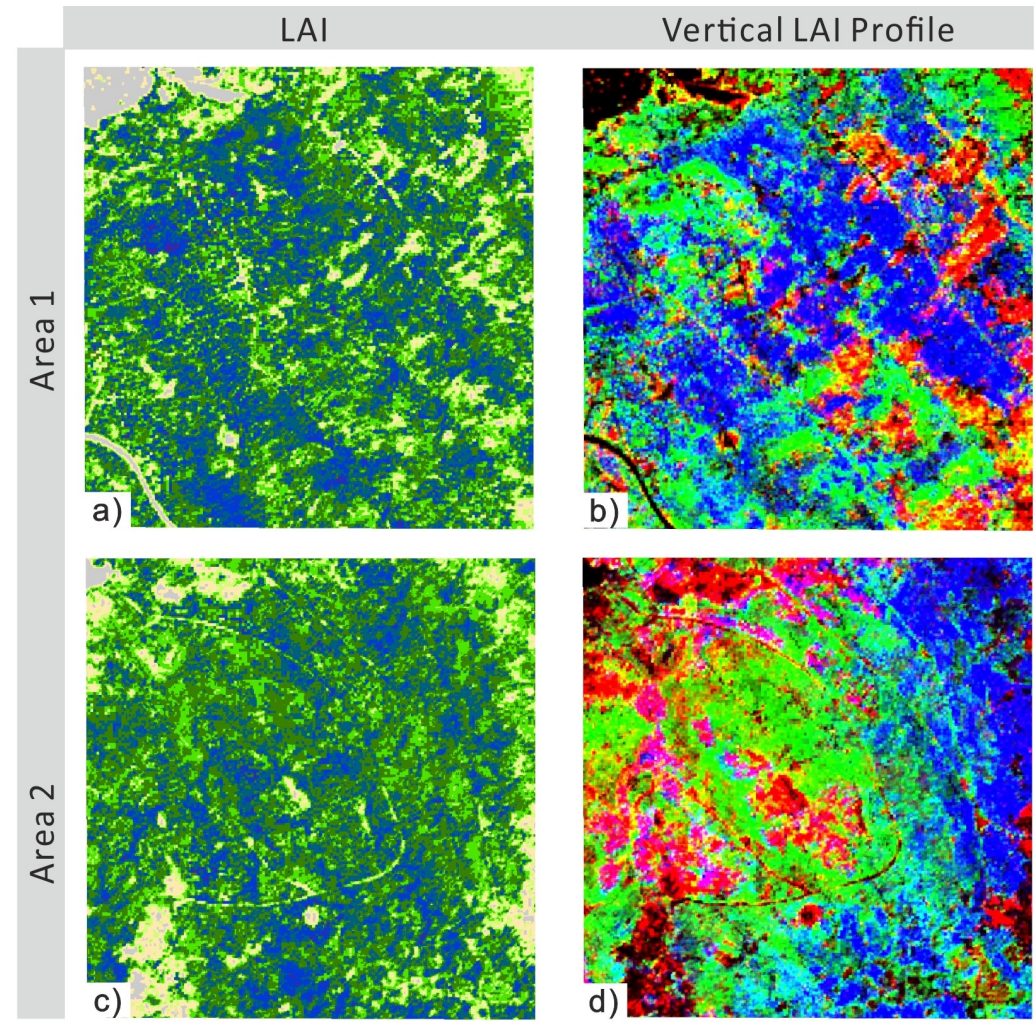

Figure 6.5: Two forest areas with similar LAI ranges but distinct vertical LAI distribution pattern revealed by airborne LiDAR (for legend please refer to Fig 6.2 and 6.4)

\subsection{Factors to account for in LAI and vertical LAI profile upscaling}

\subsubsection{Leaf angle distribution}

Leaf angle distribution (LAD) is a major source of LAI uncertainty due to its large variation in natural forests. Accurate LAD estimation becomes much more important at regional scale than at local scale. This is caused by the different scan patterns between terrestrial and airborne LiDAR. Unlike terrestrial LiDAR which can use the convergent angle of $57.5^{\circ}$ when $G(\theta)$ is approximated as 0.5 , airborne LiDAR often uses smaller scan angle $\left( \pm 10^{\circ}- \pm 30^{\circ}\right)$, which is beyond the $57.5^{\circ}$ range. Most existing studies have overlooked the importance of LAD and assumed a spherical LAD and a constant $G(\theta)$ of 0.5 for simplicity (Richardson et al., 2009; 
Tang et al., 2012; Fieber et al., 2015; Vincent et al., 2017). However, Chapter 3 demonstrated large LAD variation in natural beech forests. This finding proved the necessity to correct for LAD variation in regional LAI mapping.

However, LAD is a parameter very challenging to upscale. The extension of the LAD method at local scale (in Chapter 2 and 3) to regional scale is not feasible. Because the LAD from terrestrial LiDAR was calculated based on individual leaf reconstruction. While this is possible for dense terrestrial point clouds with small footprint (e.g. $0.15 \mathrm{~cm}-1.05 \mathrm{~cm}$ ), airborne LiDAR has much larger footprint (e.g. $0.32 \mathrm{~m}-25 \mathrm{~m}$ ) than normal leaf size. Nevertheless, there are two possible solutions which may alleviate the LAD variation problem. The first is to use terrestrial LiDAR to collect LAD reference at local scale to calibrate the airborne model. This is the strategy employed in this thesis. Based on findings in Chapter 3 , instead of a spherical LAD, a uniform LAD type was used for young and medium beech forests and a planophile LAD type was applied to mature beech forests. An alternative approach may be to use canopy height to predict LAD. As shown in Chapter 3, there is a high negative correlation between median canopy height and plot average leaf inclination angle $(r=-0.70, p<0.001$ ). This offers the potential to predict plot-specific LAD using canopy height information acquired by airborne LiDAR.

\subsubsection{Airborne LiDAR scan angle}

Airborne LiDAR data with large off-nadir scan angle was suggested to be avoided for regional LAI and vertical LAI profile mapping. The accuracy of LAI and vertical LAI profile depends on the accuracy of $P_{g a p}$ and vertical $P_{\text {gap }}$ profile. However, Chapter 4 demonstrated the accuracy of $P_{\text {gap }}$ decreases with increasing off-nadir scan angle, and underestimation of $P_{\text {gap }}$ became more severe at large off-nadir angles. Selection of appropriate scan angle in airborne LiDAR is facing with a bit of dilemma. On the one hand, according to findings in Chapter 4, close to nadir airborne LiDAR data is preferred to ensure accurate and unbiased estimate of $P_{\text {gap }}$ (Liu et al., 2018a). On the other hand, Chapter 3 implies LAI errors induced by lack of accounting for LAD variation is more severe at smaller scan angles. As a compromise, in the LAI regional mapping in Fig 6.2 
and 6.4, only nadir $\left(0^{\circ}-7^{\circ}\right)$ and small off-nadir $\left(7^{\circ}-23^{\circ}\right)$ airborne LiDAR data were used, while large off-nadir $\left(>23^{\circ}\right)$ data were excluded.

Further research is expected to look for the optimal airborne LiDAR flight settings for LAI and vertical LAI profile mapping. Especially for forest change monitoring using multi-temporal LiDAR data, such as fire severity or insect attack assessment (Solberg et al., 2006; Ma, 2018), it is necessary to use similar flight settings or correct for the uncertainty induced by different scan angles.

\subsubsection{Other factors}

Correcting for woody elements is more difficult for regional LAI mapping using airborne LiDAR than for local LAI mapping using terrestrial LiDAR. Although the differentiation of foliage and woody elements have been accomplished with high accuracy for both broadleaf and coniferous trees (Zhu et al., 2018a), extrapolation of the method to airborne LiDAR is not viable. Due to the larger footprint size of airborne LiDAR, foliage and woody materials are often mixed in the same return and inseparable. Therefore, more strictly speaking, the LAI and vertical LAI profile in this thesis and many other studies are indeed PAI and vertical PAI profile. Fortunately, the contribution of woody elements may not be high. Results from a Monte Carlo simulation have proved when seeing the canopy from nadir direction, the viewed leaf area is much larger than woody components (Hancock et al., 2012). For deciduous trees, the woody elements contribution may be corrected to a certain extent by combination of leaf-on and leaf-off airborne LiDAR flights.

Pulse density is another factor which may affect the LAI and vertical LAI profile retrieval. In this thesis, airborne LiDAR data with pulse density lower than $3 \mathrm{pl} / \mathrm{m}^{2}$ were excluded. This threshold is above the recommendation of $1 \mathrm{pl} / \mathrm{m}^{2}$ for canopy cover metrics mapping in previous studies (Jakubowski et al., 2013). 


\section{Retrieving LAI and vertical LAI profile at continental or global scale}

\subsection{Integration of satellite LiDAR with passive optical sensors}

In order to acquire wall-to-wall or gridded mapping of LAI and vertical LAI profile at continental or global scale, integration of satellite LiDAR with passive optical sensors is inevitable. Unlike passive optical sensors, satellite LiDAR has sparse coverage of the earth. An example of the GLAS (on board the ICESAT) data coverage in California in 2005 is shown in Fig 6.6. The footprint size and spacing of GEDI, a high resolution spaceborne LiDAR which was succesfully launched in Dec 2018, is displayed in Fig 6.7. The limitation of sparse coverage requires integration with ancillary passive optical dataset such as MODIS or Landsat. A similar approach has been utilized to generate several global canopy height maps (Lefsky, 2010; Simard et al., 2011) and biomass maps (Saatchi et al., 2011). It can be employed in LAI and vertical LAI profile mapping as well.

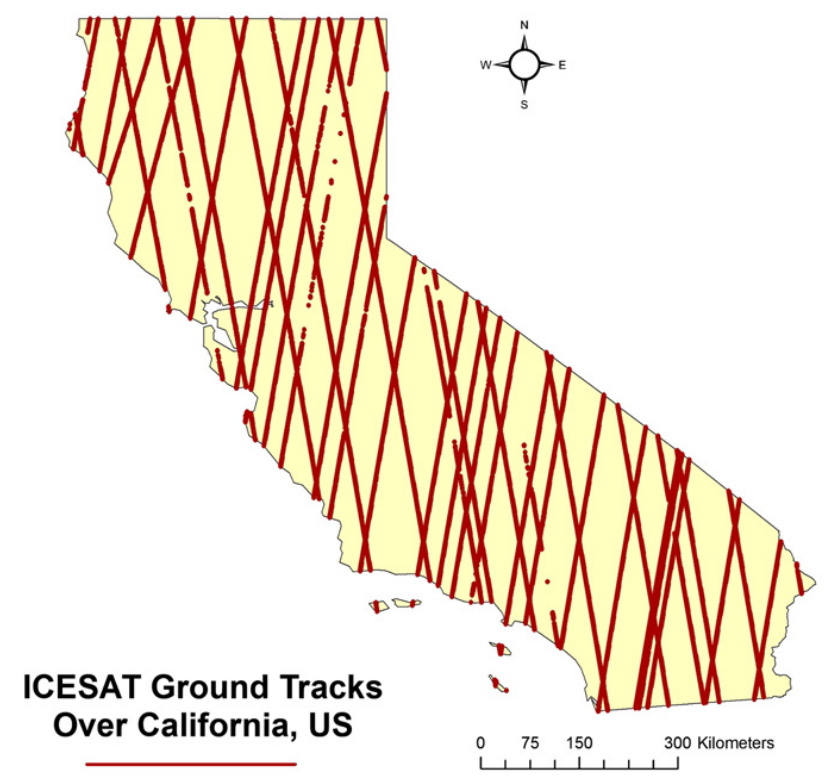

Figure 6.6: The coverage of GLAS data in California from 2003-2007 (Tang et al., 2014b) 


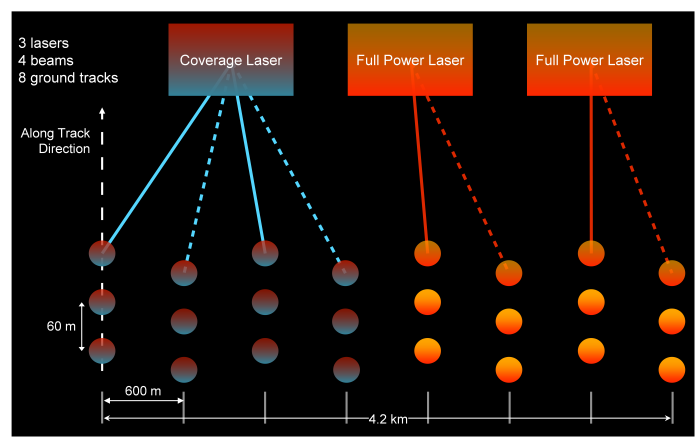

Figure 6.7: The footprint size and spacing of GEDI (Dubayah et al., 2014)

\subsection{Product validation using terrestrial and airborne LiDAR}

LAI and vertical LAI profile retrieved from terrestrial LiDAR has been used as reference for validating vertical LAI profile product retrieved from satellite LiDAR (Tang et al., 2014a,b). The autonomously operating terrestrial LiDAR enables efficient collection of hyper-temporal in-situ reference (Eitel et al., 2016). In the absence of terrestrial data, airborne LiDAR serve as an alternative for satellite product validation (Zhao and Popescu, 2009; Tang et al., 2014a). The multiple free airborne LiDAR data available in recent years offers the possibility of setting up a global validation network. Existing open airborne LiDAR data can be found through the USGS 3D elevation program (Sugarbaker et al., 2014), the US national ecological observatory network (Kampe et al., 2010), the European facility for airborne research (www.eufar.net) and the Australian terrestrial ecosystem research network (https://www.tern.org.au/).

It should be noted that in validating satellite vertical LAI profile product, topographic normalization is suggested to be avoided on reference values from airborne or terrestrial LiDAR data. In this thesis, Chapter 5 demonstrated the common preprocessing step of topographic normalization, lead to large distortion and offset of the vertical LAI profile in mountainous forests. Since satellite LiDAR has large footprint $(\sim 25 \mathrm{~m}$ in diameter for GEDI), the local topography could not be resolved or normalized. Similarly, topographic normalization should not be used on airborne or terrestrial LiDAR data. Otherwise in rugged topography, the offset of vertical LAI profiles may lead to poor validation accuracy. This 
may explain the poorer agreement between satellite and airborne LiDAR, in the vertical LAI profile product $\left(R^{2}=0.36, \mathrm{RMSE}=0.26\right)$ than in the LAI product $\left(R^{2}=0.60\right.$, RMSE $\left.=0.82\right)$ (Tang et al., 2016).

\section{Broader applications}

\subsection{Carbon stock modelling}

A significant improvement in the estimation of terrestrial carbon and water flux should be expected by incorporating the vertical LAI profile rather than the LAI alone (Tang, 2015). This has been highlighted in a pioneer study of gross primary production (GPP) modelling (Kotchenova et al., 2004). It was proved the vertical LAI profile facilitates more accurate modelling of incident direct and diffuse photosynthetically active radiation (PAR, $400-700 \mathrm{~nm}$ ). It also allows for more accurate modelling of sunlit and shaded leaves distribution inside the canopy. Furthermore, the vertical variation of LAD retrieved from LiDAR, can be used as input parameter in multi-layered radiative transfer models, to better estimate photosynthesis and evapotranspiration in vertical heterogeneous canopies (Yang et al., 2017).

\subsection{Forest dynamics monitoring}

Using multi temporal LiDAR data, the changes of vertical LAI profile enhance our capability to monitor forest 3D dynamics and understand forest growth. For instance, the dry season green-up of Amazon forests has been under debate about whether it is radiation induced forest growth or just an illusion artifact due to changeable remote sensing observation angles. Using vertical LAI profiles retrieved from satellite LiDAR, Tang and Dubayah (2017) provided evidence of understory leaf growth in dry season. Combined with environmental factors such as temperature, elevation, precipitation and soil moisture, it was proved the understory growth was driven by seasonal variations of light-regime rather than precipitation. Using hyper temporal measurements, even the rapid and subtle changes in vertical forest structure can be acquired. For instance, using the vertical LAI profile product, different start of season 
(SOS) days were automatically observed for leaves at different canopy layers (Calders et al., 2015), which is critical for forest phenology and physiology study (Calders et al., 2015; Griebel et al., 2015).

\subsection{Biodiversity modelling}

In addition to LAI, the vertical LAI profile may be a potential essential biodiversity variable. From the last century, biodiversity has been declining at an unprecedented rate as a complex response to human-induced changes in the global environment (Barnosky et al., 2011). Reducing the rate of biodiversity loss are international goals (Pereira et al., 2013). In forest ecosystems, biodiversity indicators based on structures are generating considerable interest both in their role as practical surrogates and as a key to understanding the sources of biodiversity (McElhinny et al., 2005).

Many species including birds (Walther, 2002; Siegel and DeSante, 2003), primates (Davies et al., 2017), arthropods (Halaj et al., 2000), and reptiles (Shine et al., 2002) were reported to have structural preferences in forests. The vertical LAI profile product, can be used as an input parameter for habitat modelling of these species. The complexity of forest vertical structure can account for patterns of animal diversity and richness (MacArthur and MacArthur, 1961; DeVries et al., 1997). Intensive studies have established the positive correlation between habitat structure complexity and species biodiversity (MacArthur and MacArthur, 1961; Clawges et al., 2008). The rationale for this is that more structurally complex environments provide a variety of resources and increased niche space, thus facilitating specialization and avoidance of competition through spatial segregation (Cramer and Willig, 2005). A mechanism of niche partitioning along the vertical axis has also been proposed Kalko and Handley (2001). Therefore, the vertical LAI profile map from LiDAR is promising to be applied in forest vertical structure fragmentation and complexity modelling. The resulting complexity map may greatly help identify biodiversity hotspots for conservation practice.

Overall, this thesis focused on improving the accuracy of LAI and vertical LAI profile retrieval at local and regional scales. The method in this thesis 
can be transferred to other species and sites. In the broader context, the three dimensional (3D) distribution of leaves retrieved from multiscale LiDAR create numerous applications in carbon stock modelling, forest dynamics monitoring, and biodiversity modelling. 



\section{Bibliography}

Allee, W.C., Park, O., Emerson, A.E., Park, T., Schmidt, K.P., 1949. Principles of animal ecology. WB Saundere Co. Ltd.

de Almeida, D.R.A., Nelson, B.W., Schietti, J., Gorgens, E.B., Resende, A.F., Stark, S.C., Valbuena, R., 2016. Contrasting fire damage and fire susceptibility between seasonally flooded forest and upland forest in the Central Amazon using portable profiling LiDAR. Remote Sensing of Environment 184, 153-160.

Alonzo, M., Bookhagen, B., McFadden, J.P., Sun, A., Roberts, D.A., 2015. Mapping urban forest leaf area index with airborne lidar using penetration metrics and allometry. Remote Sensing of Environment 162, 141-153.

Anderson, R., Loucks, O., Swain, A.M., 1969. Herbaceous response to canopy cover, light intensity, and throughfall precipitation in coniferous forests. Ecology 50, 255-263.

Armston, J., Disney, M., Lewis, P., Scarth, P., Phinn, S., Lucas, R., Bunting, P., Goodwin, N., 2013. Direct retrieval of canopy gap probability using airborne waveform LiDAR. Remote Sensing of Environment 134, 24-38.

Asner, G.P., 1998. Biophysical and biochemical sources of variability in canopy reflectance. Remote Sensing of Environment 64, 234-253.

Asner, G.P., Scurlock, J.M.O., Hicke, J.A., 2003. Global synthesis of leaf area index observations: implications for ecological and remote sensing studies. Global Ecology and Biogeography 12, 191-205.

Atkins, J.W., Bohrer, G., Fahey, R.T., Hardiman, B.S., Morin, T.H., Stovall, A.E., Zimmerman, N., Gough, C.M., 2018. Quantifying vegetation and canopy structural complexity from terrestrial LiDAR data using the FORESTR R package. Methods in Ecology and Evolution 9, 2057-2066. Atzberger, C., Richter, K., 2012. Spatially constrained inversion of radiat- 
ive transfer models for improved LAI mapping from future Sentinel-2 imagery. Remote Sensing of Environment 120, 208-218.

Bacour, C., Baret, F., Béal, D., Weiss, M., Pavageau, K., 2006. Neural network estimation of LAI, fAPAR, fCover and LAI $\times$ Cab, from top of canopy MERIS reflectance data: Principles and validation. Remote sensing of environment 105, 313-325.

Bailey, B.N., Mahaffee, W.F., 2017. Rapid measurement of the threedimensional distribution of leaf orientation and the leaf angle probability density function using terrestrial LiDAR scanning. Remote Sensing of Environment 194, 63-76.

Balandier, P., Sinoquet, H., Frak, E., Giuliani, R., Vandame, M., Descamps, S., Coll, L., Adam, B., Prevosto, B., Curt, T., 2007. Six-year time course of light-use efficiency, carbon gain and growth of beech saplings (Fagus sylvatica) planted under a Scots pine (Pinus sylvestris) shelterwood. Tree Physiology 27, 1073-1082.

Baltsavias, E.P., 1999. Airborne laser scanning: basic relations and formulas. ISPRS Journal of Photogrammetry and Remote Sensing 54, 199-214.

Baret, F., Weiss, M., Lacaze, R., Camacho, F., Makhmara, H., Pacholcyzk, P., Smets, B., 2013. GEOV1: LAI and FAPAR essential climate variables and FCOVER global time series capitalizing over existing products. Part1: Principles of development and production. Remote Sensing of Environment 137, 299-309.

Barna, M., 2004. Adaptation of European beech(Fagus sylvatica L.) to different ecological conditions: leaf size variation. Polish Journal of Ecology 52, 35-45.

Barnosky, A.D., Matzke, N., Tomiya, S., Wogan, G.O., Swartz, B., Quental, T.B., Marshall, C., McGuire, J.L., Lindsey, E.L., Maguire, K.C., et al., 2011. Has the Earth's sixth mass extinction already arrived? Nature 471, 51.

Barr, A.G., Black, T.A., Hogg, E.H., Kljun, N., Morgenstern, K., Nesic, Z., 2004. Inter-annual variability in the leaf area index of a boreal aspenhazelnut forest in relation to net ecosystem production. Agricultural and Forest Meteorology 126, 237-255.

Bässler, C., Förster, B., Moning, C., Müller, J., 2008. The BIOKLIM-Project: biodiversity research between climate change and wilding in a temperate montane forest-the conceptual framework. Waldökologie, Landschaftsforschung und Naturschutz 7, 21-33. 
Bater, C.W., Coops, N.C., 2009. Evaluating error associated with lidarderived DEM interpolation. Computers \& Geosciences 35, 289-300.

Bayat, B., Van Der Tol, C., Verhoef, W., 2018. Integrating satellite optical and thermal infrared observations for improving daily ecosystem functioning estimations during a drought episode. Remote Sensing of Environment 209, 375-394.

Beaudet, M., Messier, C., 2002. Variation in canopy openness and light transmission following selection cutting in northern hardwood stands: an assessment based on hemispherical photographs. Agricultural and Forest Meteorology 110, 217-228.

Bechtold, S., Höfle, B., 2016. HELIOS: A multi-purpose LiDAR simulation framework for research, planning and training of laser scanning operations with airborne, ground-based mobile and stationary platforms. ISPRS Annals of Photogrammetry, Remote Sensing \& Spatial Information Sciences 3.

Beland, M., Baldocchi, D.D., Widlowski, J.L., Fournier, R.A., Verstraete, M.M., 2014. On seeing the wood from the leaves and the role of voxel size in determining leaf area distribution of forests with terrestrial LiDAR. Agricultural and Forest Meteorology 184, 82-97.

Béland, M., Widlowski, J.L., Fournier, R.A., Côté, J.F., Verstraete, M.M., 2011. Estimating leaf area distribution in savanna trees from terrestrial LiDAR measurements. Agricultural and Forest Meteorology 151, $1252-$ 1266.

Bergen, K., Goetz, S., Dubayah, R., Henebry, G., Hunsaker, C., Imhoff, M., Nelson, R., Parker, G., Radeloff, V., 2009. Remote sensing of vegetation 3-D structure for biodiversity and habitat: Review and implications for lidar and radar spaceborne missions. Journal of Geophysical Research: Biogeosciences 114.

Biskup, B., Scharr, H., Schurr, U., Rascher, U., 2007. A stereo imaging system for measuring structural parameters of plant canopies. Plant, Cell \& Environment 30, 1299-1308.

Bojinski, S., Verstraete, M., Peterson, T.C., Richter, C., Simmons, A., Zemp, M., 2014. The concept of essential climate variables in support of climate research, applications, and policy. Bulletin of the American Meteorological Society 95, 1431-1443.

Bréda, N.J., 2003. Ground-based measurements of leaf area index: a review of methods, instruments and current controversies. Journal of 
Experimental Botany 54, 2403-2417.

Brokaw, N., Lent, R., 1999. Vertical structure. Cambridge University Press. pp. 373-399.

Cailleret, M., Heurich, M., Bugmann, H., 2014. Reduction in browsing intensity may not compensate climate change effects on tree species composition in the Bavarian Forest National Park. Forest Ecology and Management 328, 179-192.

Calders, K., Armston, J., Newnham, G., Herold, M., Goodwin, N., 2014. Implications of sensor configuration and topography on vertical plant profiles derived from terrestrial LiDAR. Agricultural and Forest Meteorology 194, 104-117.

Calders, K., Origo, N., Disney, M., Nightingale, J., Woodgate, W., Armston, J., Lewis, P., 2018. Variability and bias in active and passive groundbased measurements of effective plant, wood and leaf area index. Agricultural and Forest Meteorology 252, 231-240.

Calders, K., Schenkels, T., Bartholomeus, H., Armston, J., Verbesselt, J., Herold, M., 2015. Monitoring spring phenology with high temporal resolution terrestrial LiDAR measurements. Agricultural and Forest Meteorology 203, 158-168.

Campbell, G., 1986. Extinction coefficients for radiation in plant canopies calculated using an ellipsoidal inclination angle distribution. Agricultural and Forest Meteorology 36, 317-321.

Campbell, G., Norman, J., et al., 1989. The description and measurement of plant canopy structure. volume 31. Cambridge University Press Cambridge.

Canham, C.D., Denslow, J.S., Platt, W.J., Runkle, J.R., Spies, T.A., White, P.S., 1990. Light regimes beneath closed canopies and tree-fall gaps in temperate and tropical forests. Canadian Journal of Forest Research 20, 620-631.

Cao, L., Coops, N.C., Innes, J.L., Sheppard, S.R., Fu, L., Ruan, H., She, G., 2016. Estimation of forest biomass dynamics in subtropical forests using multi-temporal airborne LiDAR data. Remote Sensing of Environment 178, 158-171.

Chen, J., Black, T., Adams, R., 1991. Evaluation of hemispherical photography for determining plant area index and geometry of a forest stand. Agricultural and Forest Meteorology 56, 129-143.

Chen, J.M., Black, T.A., 2010. Defining leaf area index for non-flat leaves. 
Plant Cell \& Environment 15, 421-429.

Chen, J.M., Rich, P.M., Gower, S.T., Norman, J.M., Plummer, S., 1997. Leaf area index of boreal forests: Theory, techniques, and measurements. Journal of Geophysical Research: Atmospheres 102, 29429-29443.

Chen, X., Disney, M., Lewis, P., Armston, J., Han, J., Li, J., 2014. Sensitivity of direct canopy gap fraction retrieval from airborne waveform lidar to topography and survey characteristics. Remote Sensing of Environment $143,15-25$.

Chen, Y., Zhang, W., Hu, R., Qi, J., Shao, J., Li, D., Wan, P., Qiao, C., Shen, A., Yan, G., 2018. Estimation of forest leaf area index using terrestrial laser scanning data and path length distribution model in open-canopy forests. Agricultural and Forest Meteorology 263, 323-333.

Chianucci, F., Cutini, A., Corona, P., Puletti, N., 2014. Estimation of leaf area index in understory deciduous trees using digital photography. Agricultural and Forest Meteorology 198, 259-264.

Chianucci, F., Macfarlane, C., Pisek, J., Cutini, A., Casa, R., 2015. Estimation of foliage clumping from the LAI-2000 Plant Canopy Analyzer: effect of view caps. Trees 29, 355-366.

Chianucci, F., Pisek, J., Raabe, K., Marchino, L., Ferrara, C., Corona, P., 2018. A dataset of leaf inclination angles for temperate and boreal broadleaf woody species. Annals of Forest Science 75 .

Clawges, R., Vierling, K., Vierling, L., Rowell, E., 2008. The use of airborne lidar to assess avian species diversity, density, and occurrence in a pine/aspen forest. Remote Sensing of Environment 112, 2064-2073.

Contreras, M.A., Parsons, R.A., Chung, W., 2012. Modeling tree-level fuel connectivity to evaluate the effectiveness of thinning treatments for reducing crown fire potential. Forest Ecology and Management 264, 134-149.

Coops, N.C., Hilker, T., Wulder, M.A., St-Onge, B., Newnham, G., Siggins, A., Trofymow, J.T., 2007. Estimating canopy structure of Douglas-fir forest stands from discrete-return LiDAR. Trees 21, 295-310.

Cramer, M.J., Willig, M.R., 2005. Habitat heterogeneity, species diversity and null models. Oikos 108, 209-218.

Crockford, R.H., Richardson, D.P., 2010. Partitioning of rainfall into throughfall, stemflow and interception: effect of forest type, ground cover and climate. Hydrological Processes 14, 2903-2920.

Danson, F.M., Disney, M.I., Gaulton, R., Schaaf, C., Strahler, A., 2018. The 
terrestrial laser scanning revolution in forest ecology. Interface Focus 8.

Danson, F.M., Hetherington, D., Morsdorf, F., Koetz, B., Allgower, B., 2007. Forest canopy gap fraction from terrestrial laser scanning. IEEE Geoscience and Remote Sensing Letters 4, 157-160.

Davies, A.B., Ancrenaz, M., Oram, F., Asner, G.P., 2017. Canopy structure drives orangutan habitat selection in disturbed Bornean forests. Proceedings of the National Academy of Sciences 114, 8307-8312.

Delagrange, S., Montpied, P., Dreyer, E., Messier, C., Sinoquet, H., 2006. Does shade improve light interception efficiency? A comparison among seedlings from shade-tolerant and -intolerant temperate deciduous tree species. New Phytologist 172, 293-304.

Demantke, J., Mallet, C., David, N., Vallet, B., 2011. Dimensionality based scale selection in 3D lidar point clouds. The International Archives of the Photogrammetry, Remote Sensing and Spatial Information Sciences 38, W12.

DeVries, P.J., Murray, D., Lande, R., 1997. Species diversity in vertical, horizontal, and temporal dimensions of a fruit-feeding butterfly community in an Ecuadorian rainforest. Biological journal of the Linnean Society 62, 343-364.

Drake, J.B., Dubayah, R.O., Clark, D.B., Knox, R.G., Blair, J.B., Hofton, M.A., Chazdon, R.L., Weishampel, J.F., Prince, S., 2002. Estimation of tropical forest structural characteristics using large-footprint lidar. Remote Sensing of Environment 79, 305-319.

Dubayah, R., Goetz, S., Blair, J., Fatoyinbo, T., Hansen, M., Healey, S., Hofton, M., Hurtt, G., Kellner, J., Luthcke, S., et al., 2014. The global ecosystem dynamics investigation, in: AGU Fall Meeting Abstracts.

Eitel, J.U., Magney, T.S., Vierling, L.A., Greaves, H.E., Zheng, G., 2016. An automated method to quantify crop height and calibrate satellitederived biomass using hypertemporal lidar. Remote Sensing of Environment 187, 414-422.

Eitel, J.U.H., Vierling, L.A., Long, D.S., 2010. Simultaneous measurements of plant structure and chlorophyll content in broadleaf saplings with a terrestrial laser scanner. Remote Sensing of Environment 114, 22292237.

Ellenberg, H., Leuschner, C., 2010. Vegetation Mitteleuropas mit den Alpen: in ökologischer, dynamischer und historischer Sicht. volume 
8104. Utb.

Falkowski, M.J., Evans, J.S., Martinuzzi, S., Gessler, P.E., Hudak, A.T., 2009. Characterizing forest succession with lidar data: An evaluation for the Inland Northwest, USA. Remote Sensing of Environment 113, 946-956.

Falster, D.S., Westoby, M., 2003. Leaf size and angle vary widely across species: what consequences for light interception? New Phytologist 158, 509-525.

Fang, H.L., Jiang, C.Y., Li, W.J., Wei, S.S., Baret, F., Chen, J.M., Garcia-Haro, J., Liang, S.L., Liu, R.G., Myneni, R.B., Pinty, B., Xiao, Z.Q., Zhu, Z.C., 2013. Characterization and intercomparison of global moderate resolution leaf area index (LAI) products: Analysis of climatologies and theoretical uncertainties. Journal of Geophysical Research: Biogeosciences 118, 529-548.

Ferrara, R., Virdis, S.G.P., Ventura, A., Ghisu, T., Duce, P., Pellizzaro, G., 2018. An automated approach for wood-leaf separation from terrestrial LIDAR point clouds using the density based clustering algorithm DBSCAN. Agricultural and Forest Meteorology 262, 434-444.

Ferreira, M.P., Feret, J.B., Grau, E., Gastellu-Etchegorry, J.P., Shimabukuro, Y.E., de Souza, C.R., 2018. Retrieving structural and chemical properties of individual tree crowns in a highly diverse tropical forest with 3D radiative transfer modeling and imaging spectroscopy. Remote Sensing of Environment 211, 276-291.

Fieber, K.D., Davenport, I.J., Tanase, M.A., Ferryman, J.M., Gurney, R.J., Becerra, V.M., Walker, J.P., Hacker, J.M., 2015. Validation of Canopy Height Profile methodology for small-footprint full-waveform airborne LiDAR data in a discontinuous canopy environment. ISPRS Journal of Photogrammetry and Remote Sensing 104, 144-157.

Foody, G.M., 1996. Approaches for the production and evaluation of fuzzy land cover classifications from remotely-sensed data. International Journal of Remote Sensing 17, 1317-1340.

Foody, G.M., Mathur, A., 2004. A relative evaluation of multiclass image classification by support vector machines. IEEE Transactions on Geoscience and Remote Sensing 42, 1335-1343.

Fournier, R.A., Hall, R.J., 2017. Hemispherical Photography in Forest Science: Theory, Methods, Applications. volume 28. Springer.

Franklin, J.F., Spies, T.A., 1991. Composition, function, and structure of old-growth Douglas-fir forests. Wildlife and Vegetation of Unmanaged 
Douglas-fir Forests. USDA Forest Service General Technical Report PNW-GTR-285, 71-80.

Frazer, G.W., Fournier, R.A., Leblanc, S.G., Walter, J.M.N., 2017. View angledependent clumping indices for Indirect LAI estimation. Springer. pp. 153-185.

Garcia, M., Saatchi, S., Ferraz, A., Silva, C.A., Ustin, S., Koltunov, A., Balzter, H., 2017. Impact of data model and point density on aboveground forest biomass estimation from airborne LiDAR. Carbon balance and management 12, 4.

Garrigues, S., Shabanov, N., Swanson, K., Morisette, J., Baret, F., Myneni, R., 2008. Intercomparison and sensitivity analysis of Leaf Area Index retrievals from LAI-2000, AccuPAR, and digital hemispherical photography over croplands. Agricultural and Forest Meteorology 148, 1193-1209.

Gastellu-Etchegorry, J.P., Yin, T., Lauret, N., Cajgfinger, T., Gregoire, T., Grau, E., Feret, J.B., Lopes, M., Guilleux, J., Dedieu, G., Malenovsky, Z., Cook, B., Morton, D., Rubio, J., Durrieu, S., Cazanave, G., Martin, E., Ristorcelli, T., 2015. Discrete Anisotropic Radiative Transfer (DART 5) for Modeling Airborne and Satellite Spectroradiometer and LIDAR Acquisitions of Natural and Urban Landscapes. Remote Sensing 7, 1667.

Geißler, C., Kühn, P., Böhnke, M., Bruelheide, H., Shi, X., Scholten, T., 2012. Splash erosion potential under tree canopies in subtropical SE China. Catena 91, 85-93.

Girardeau-Montaut, D., 2015. Cloud compare-3d point cloud and mesh processing software. Open Source Project .

Goetz, S., Steinberg, D., Dubayah, R., Blair, B., 2007. Laser remote sensing of canopy habitat heterogeneity as a predictor of bird species richness in an eastern temperate forest, USA. Remote Sensing of Environment 108, 254-263.

Gonsamo, A., Pellikka, P., 2008. Methodology comparison for slope correction in canopy leaf area index estimation using hemispherical photography. Forest Ecology and Management 256, 749-759.

Gray, A.N., Spies, T.A., 1996. Gap size, within-gap position and canopy structure effects on conifer seedling establishment. Journal of Ecology , 635-645.

Greaves, H.E., Vierling, L.A., Eitel, J.U.H., Boelman, N.T., Magney, T.S., 
Prager, C.M., Griffin, K.L., 2015. Estimating aboveground biomass and leaf area of low-stature Arctic shrubs with terrestrial LiDAR. Remote Sensing of Environment 164, 26-35.

Griebel, A., Bennett, L.T., Culvenor, D.S., Newnham, G.J., Arndt, S.K., 2015. Reliability and limitations of a novel terrestrial laser scanner for daily monitoring of forest canopy dynamics. Remote Sensing of Environment 166, 205-213.

Grohmann, C.H., Smith, M.J., Riccomini, C., 2011. Multiscale analysis of topographic surface roughness in the Midland Valley, Scotland. IEEE Transactions on Geoscience and Remote Sensing 49, 1200-1213.

Gu, C., Clevers, J.G., Liu, X., Tian, X., Li, Z., Li, Z., 2018. Predicting forest height using the GOST, Landsat 7 ETM+, and airborne LiDAR for sloping terrains in the Greater Khingan Mountains of China. ISPRS Journal of Photogrammetry and Remote Sensing 137, 97-111.

Haala, N., Brenner, C., 1999. Extraction of buildings and trees in urban environments. ISPRS Journal of Photogrammetry and Remote Sensing $54,130-137$.

Halaj, J., Ross, D.W., Moldenke, A.R., 2000. Importance of habitat structure to the arthropod food-web in Douglas-fir canopies. Oikos 90, 139-152.

Hämmerle, M., Lukač, N., Chen, K.C., Koma, Z., Wang, C.K., Anders, K., Höfle, B., 2017. Simulating various terrestrial and UAV LiDAR scanning configurations for understory forest structure modelling. ISPRS Annals of Photogrammetry, Remote Sensing \& Spatial Information Sciences 4. Hancock, S., Armston, J., Li, Z., Gaulton, R., Lewis, P., Disney, M., Danson, F.M., Strahler, A., Schaaf, C., Anderson, K., 2015. Waveform lidar over vegetation: An evaluation of inversion methods for estimating return energy. Remote Sensing of Environment 164, 208-224.

Hancock, S., Essery, R., Reid, T., Carle, J., Baxter, R., Rutter, N., Huntley, B., 2014. Characterising forest gap fraction with terrestrial lidar and photography: An examination of relative limitations. Agricultural and Forest Meteorology 189, 105-114.

Hancock, S., Lewis, P., Foster, M., Disney, M., Muller, J.P., 2012. Measuring forests with dual wavelength lidar: A simulation study over topography. Agricultural and Forest Meteorology 161, 123-133.

Hansen, M.C., Potapov, P.V., Moore, R., Hancher, M., Turubanova, S.A., Tyukavina, A., Thau, D., Stehman, S.V., Goetz, S.J., Loveland, T.R., 
Kommareddy, A., Egorov, A., Chini, L., Justice, C.O., Townshend, J.R.G., 2013. High-Resolution Global Maps of 21st-Century Forest Cover Change. Science 342, 850-853.

Harding, D., Lefsky, M., Parker, G., Blair, J., 2001. Laser altimeter canopy height profiles: Methods and validation for closed-canopy, broadleaf forests. Remote Sensing of Environment 76, 283-297.

Hedstrom, N., Pomeroy, J., 1998. Measurements and modelling of snow interception in the boreal forest. Hydrological Processes 12, 16111625.

Heurich, M., Baierl, F., GÃijnther, S., Sinner, K.F., 2011. Management and conservation of large mammals in the Bavarian Forest National Park. Silva Gabreta 17, 1-18.

Heurich, M., Beudert, B., Rall, H., Křenová, Z., 2010. National parks as model regions for interdisciplinary long-term ecological research: the Bavarian Forest and Šumavá National Parks underway to transboundary ecosystem research. Springer. pp. 327-344.

Hilker, T., van Leeuwen, M., Coops, N.C., Wulder, M.A., Newnham, G.J., Jupp, D.L., Culvenor, D.S., 2010. Comparing canopy metrics derived from terrestrial and airborne laser scanning in a Douglas-fir dominated forest stand. Trees 24, 819-832.

Hill, R., Broughton, R.K., 2009. Mapping the understorey of deciduous woodland from leaf-on and leaf-off airborne LiDAR data: A case study in lowland Britain. ISPRS Journal of Photogrammetry and Remote Sensing 64, 223-233.

Höfle, B., Pfeifer, N., 2007. Correction of laser scanning intensity data: Data and model-driven approaches. ISPRS journal of photogrammetry and remote sensing 62, 415-433.

Holder, C.D., 2012. The relationship between leaf hydrophobicity, water droplet retention, and leaf angle of common species in a semi-arid region of the western United States. Agricultural and Forest Meteorology 152, 11-16.

Hollinger, D., 1989. Canopy organization and foliage photosynthetic capacity in a broad-leaved evergreen montane forest. Functional Ecology , 53-62.

Holmgren, J., Nilsson, M., Olsson, H., 2003. Simulating the effects of lidar scanning angle for estimation of mean tree height and canopy closure. Canadian Journal of Remote Sensing 29, 623-632. 
Hopkins, R., Schmitt, J., Stinchcombe, J.R., 2008. A latitudinal cline and response to vernalization in leaf angle and morphology in Arabidopsis thaliana (Brassicaceae). New Phytologist 179, 155-164.

Hopkinson, C., Chasmer, L., 2007. Modelling canopy gap fraction from lidar intensity, in: ISPRS Workshop on Laser Scanning 2007 and SilviLaser 2007, IAPRS Espoo, Finland. pp. 190-194.

Hoppe, H., DeRose, T., Duchamp, T., McDonald, J., Stuetzle, W., 1992. Surface reconstruction from unorganized points. volume 26. ACM.

Hosoi, F., Omasa, K., 2009. Detecting seasonal change of broad-leaved woody canopy leaf area density profile using 3D portable LIDAR imaging. Functional Plant Biology 36, 998-1005.

Hosoi, F., Omasa, K., 2012. Estimation of vertical plant area density profiles in a rice canopy at different growth stages by high-resolution portable scanning lidar with a lightweight mirror. ISPRS Journal of Photogrammetry and Remote Sensing 74, 11-19.

Houborg, R., Soegaard, H., Boegh, E., 2007. Combining vegetation index and model inversion methods for the extraction of key vegetation biophysical parameters using Terra and Aqua MODIS reflectance data. Remote Sensing of Environment 106, 39-58.

Hu, R., Bournez, E., Cheng, S., Jiang, H., Nerry, F., Landes, T., Saudreau, M., Kastendeuch, P., Najjar, G., Colin, J., 2018. Estimating the leaf area of an individual tree in urban areas using terrestrial laser scanner and path length distribution model. ISPRS Journal of Photogrammetry and Remote Sensing 144, 357-368.

Humphrey, J., Hawes, C., Peace, A., Ferris-Kaan, R., Jukes, M., 1999. Relationships between insect diversity and habitat characteristics in plantation forests. Forest ecology and management 113, 11-21.

Hutchison, B., Matt, D., McMillen, R., Gross, L., Tajchman, S., Norman, J., 1986. The architecture of a deciduous forest canopy in eastern Tennessee, USA. The Journal of Ecology , 635-646.

Hyde, P., Dubayah, R., Peterson, B., Blair, J., Hofton, M., Hunsaker, C., Knox, R., Walker, W., 2005. Mapping forest structure for wildlife habitat analysis using waveform lidar: Validation of montane ecosystems. Remote Sensing of Environment 96, 427-437.

Isenburg, M., 2012. LAStools-efficient tools for LiDAR processing. Available at: http: http://www. cs. unc. edu/ isenburg/lastools/[Accessed October 9, 2012] . 
Ivanov, N., Boissard, P., Chapron, M., Andrieu, B., 1995. Computer stereo plotting for 3-D reconstruction of a maize canopy. Agricultural and Forest Meteorology 75, 85-102.

Jacquemoud, S., Bacour, C., Poilve, H., Frangi, J.P., 2000. Comparison of four radiative transfer models to simulate plant canopies reflectance: Direct and inverse mode. Remote Sensing of Environment 74, 471-481.

Jakubowski, M.K., Guo, Q.H., Kelly, M., 2013. Tradeoffs between lidar pulse density and forest measurement accuracy. Remote Sensing of Environment 130, 245-253.

Jat, M.K., Garg, P.K., Khare, D., 2008. Monitoring and modelling of urban sprawl using remote sensing and GIS techniques. International Journal of Applied Earth Observation and Geoinformation 10, 26-43.

Jensen, J.L., Humes, K.S., Vierling, L.A., Hudak, A.T., 2008. Discrete return lidar-based prediction of leaf area index in two conifer forests. Remote Sensing of Environment 112, 3947-3957.

Jiang, C.Y., Ryu, Y., Fang, H.L., Myneni, R., Claverie, M., Zhu, Z.C., 2017. Inconsistencies of interannual variability and trends in long-term satellite leaf area index products. Global Change Biology 23, 4133-4146.

Jonckheere, I., Fleck, S., Nackaerts, K., Muys, B., Coppin, P., Weiss, M., Baret, F., 2004. Review of methods for in situ leaf area index determination - Part I. Theories, sensors and hemispherical photography. Agricultural and Forest Meteorology 121, 19-35.

Jupp, D.L., Culvenor, D., Lovell, J., Newnham, G., Strahler, A., Woodcock, C., 2009. Estimating forest LAI profiles and structural parameters using a ground-based laser called 'Echidna ${ }^{\circledR}$. Tree Physiology 29, 171-181.

Kalko, E.K., Handley, C.O., 2001. Neotropical bats in the canopy: diversity, community structure, and implications for conservation. Plant ecology 153, 319-333.

Kampe, T.U., Johnson, B.R., Kuester, M.A., Keller, M., 2010. NEON: the first continental-scale ecological observatory with airborne remote sensing of vegetation canopy biochemistry and structure. Journal of Applied Remote Sensing 4, 043510.

Kattge, J., Diaz, S., Lavorel, S., Prentice, I.C., Leadley, P., Bönisch, G., Garnier, E., Westoby, M., Reich, P.B., Wright, I.J., et al., 2011. TRY-a global database of plant traits. Global Change Biology 17, 2905-2935. Kempeneers, P., Zarco-Tejada, P.J., North, P.R., de Backer, S., Delalieux, S., Sepulcre-Cantó, G., Morales, F., Van Aardt, J., Sagardoy, R., Coppin, 
P., et al., 2008. Model inversion for chlorophyll estimation in open canopies from hyperspectral imagery. International Journal of Remote Sensing 29, 5093-5111.

Khosravipour, A., Skidmore, A.K., Isenburg, M., Wang, T., Hussin, Y.A., 2014. Generating pit-free canopy height models from airborne lidar. Photogrammetric Engineering \& Remote Sensing 80, 863-872.

Khosravipour, A., Skidmore, A.K., Wang, T., Isenburg, M., Khoshelham, K., 2015. Effect of slope on treetop detection using a LiDAR Canopy Height Model. ISPRS Journal of Photogrammetry and Remote Sensing $104,44-52$.

King, D.A., 1997. The functional significance of leaf angle in Eucalyptus. Australian Journal of Botany 45, 619-639.

Klasing, K., Althoff, D., Wollherr, D., Buss, M., 2009. Comparison of surface normal estimation methods for range sensing applications, in: Robotics and Automation, 2009. ICRA'09. IEEE International Conference on, IEEE. pp. 3206-3211.

Koetz, B., Morsdorf, F., Sun, G., Ranson, K.J., Itten, K., Allgower, B., 2006. Inversion of a lidar waveform model for forest biophysical parameter estimation. IEEE Geoscience and Remote Sensing Letters 3, 49-53.

Korhonen, L., Korpela, I., Heiskanen, J., Maltamo, M., 2011. Airborne discrete-return LIDAR data in the estimation of vertical canopy cover, angular canopy closure and leaf area index. Remote Sensing of Environment 115, 1065-1080.

Kotchenova, S.Y., Song, X., Shabanov, N.V., Potter, C.S., Knyazikhin, Y., Myneni, R.B., 2004. Lidar remote sensing for modeling gross primary production of deciduous forests. Remote Sensing of Environment 92, 158-172.

Kull, O., Broadmeadow, M., Kruijt, B., Meir, P., 1999. Light distribution and foliage structure in an oak canopy. Trees-Structure and Function $14,55-64$.

Kuusk, A., 2001. A two-layer canopy reflectance model. Journal of Quantitative Spectroscopy and Radiative Transfer 71, 1-9.

Lang, A., 1973. Leaf orientation of a cotton plant. Agricultural Meteoro$\operatorname{logy} 11,37-51$.

Lang, M., Kuusk, A., Mõttus, M., Rautiainen, M., Nilson, T., 2010. Canopy gap fraction estimation from digital hemispherical images using sky radiance models and a linear conversion method. Agricultural and 
Forest Meteorology 150, 20-29.

Latifi, H., Heurich, M., Hartig, F., MÃijller, J., Krzystek, P., Jehl, H., Dech, S., 2015. Estimating over-and understorey canopy density of temperate mixed stands by airborne LiDAR data. Forestry , cpv032.

Lausch, A., Heurich, M., Fahse, L., 2013. Spatio-temporal infestation patterns of Ips typographus (L.) in the Bavarian Forest National Park, Germany. Ecological Indicators 31, 73-81.

Leblanc, S., Fournier, R., 2014. Hemispherical photography simulations with an architectural model to assess retrieval of leaf area index. Agricultural and Forest Meteorology 194, 64-76.

Leblanc, S.G., Fournier, R.A., 2017. Measurement of forest structure with hemispherical photography, in: Hemispherical photography in forest science: theory, methods, applications. Springer, pp. 53-83.

Lefsky, M., Turner, D., Guzy, M., Cohen, W., 2005. Combining lidar estimates of aboveground biomass and Landsat estimates of stand age for spatially extensive validation of modeled forest productivity. Remote Sensing of Environment 95, 549-558.

Lefsky, M.A., 2010. A global forest canopy height map from the Moderate Resolution Imaging Spectroradiometer and the Geoscience Laser Altimeter System. Geophysical Research Letters 37.

Lefsky, M.A., Cohen, W.B., Parker, G.G., Harding, D.J., 2002. Lidar remote sensing for ecosystem studies. Bioscience 52, 19-30.

Lefsky, M.A., Harding, D., Cohen, W., Parker, G., Shugart, H., 1999. Surface lidar remote sensing of basal area and biomass in deciduous forests of eastern Maryland, USA. Remote Sensing of Environment 67, 83-98.

Leiterer, R., Torabzadeh, H., Furrer, R., Schaepman, M.E., Morsdorf, F., 2015. Towards automated characterization of canopy layering in mixed temperate forests using airborne laser scanning. Forests 6, 4146-4167.

Lemeur, R., Blad, B.L., 1975. A critical review of light models for estimating the shortwave radiation regime of plant canopies, in: Developments in Agricultural and Managed Forest Ecology. Elsevier. volume 1, pp. 255-286.

Levia, D., Michalzik, B., Näthe, K., Bischoff, S., Richter, S., Legates, D., 2015. Differential stemflow yield from European beech saplings: the role of individual canopy structure metrics. Hydrological processes 29, 43-51.

Li, Y., Su, Y., Hu, T., Xu, G., Guo, Q., 2018a. Retrieving 2-D leaf angle 
distributions for deciduous trees from terrestrial laser scanner data. IEEE Transactions on Geoscience and Remote Sensing , 1-11.

Li, Z., Schaefer, M., Strahler, A., Schaaf, C., Jupp, D., 2018b. On the utilization of novel spectral laser scanning for three-dimensional classification of vegetation elements. Interface focus 8, 20170039.

Li, Z., Strahler, A., Schaaf, C., Jupp, D., Schaefer, M., Olofsson, P., 2018c. Seasonal change of leaf and woody area profiles in a midlatitude deciduous forest canopy from classified dual-wavelength terrestrial lidar point clouds. Agricultural and Forest Meteorology 262, 279-297.

Liang, X., Hyyppä, J., Kaartinen, H., Lehtomäki, M., Pyörälä, J., Pfeifer, N., Holopainen, M., Brolly, G., Francesco, P., Hackenberg, J., et al., 2018. International benchmarking of terrestrial laser scanning approaches for forest inventories. ISPRS Journal of Photogrammetry and Remote Sensing 144, 137-179.

LiCOR, 2009. LAI-2200 Plant Canopy Analyzer. Instruction Manual .

Lin, Y., West, G., 2016. Retrieval of effective leaf area index (LAIe) and leaf area density (LAD) profile at individual tree level using high density multi-return airborne LiDAR. International Journal of Applied Earth Observation and Geoinformation 50, 150-158.

Lindberg, E., Olofsson, K., Holmgren, J., Olsson, H., 2012. Estimation of $3 \mathrm{D}$ vegetation structure from waveform and discrete return airborne laser scanning data. Remote Sensing of Environment 118, 151-161.

Liu, J., Skidmore, A.K., Heurich, M., Wang, T., 2017a. Significant effect of topographic normalization of airborne LiDAR data on the retrieval of plant area index profile in mountainous forests. ISPRS journal of photogrammetry and remote sensing 132, 77-87.

Liu, J., Skidmore, A.K., Jones, S., Wang, T., Heurich, M., Zhu, X., Shi, Y., 2018a. Large off-nadir scan angle of airborne LiDAR can severely affect the estimates of forest structure metrics. ISPRS Journal of Photogrammetry and Remote Sensing 136, 13-25.

Liu, J., Skidmore, A.K., Wang, T., Zhu, X., Premier, J., Heurich, M., Beudert, B., Jones, S., 2019. Variation of leaf angle distribution quantified by terrestrial LiDAR in natural European beech forest. ISPRS journal of photogrammetry and remote sensing 148, 208-220.

Liu, J.C., Melloh, R.A., Woodcock, C.E., Davis, R.E., Painter, T.H., Mckenzie, C., 2008. Modeling the View Angle Dependence of Gap Fractions in Forest Canopies: Implications for Mapping Fractional Snow Cover Using 
Optical Remote Sensing. Journal of Hydrometeorology 9, 1005-1019.

Liu, S.Y., Baret, F., Abichou, M., Boudon, F., Thomas, S., Zhao, K.G., Fournier, C., Andrieu, B., Irfan, K., Hemmerle, M., de Solan, B., 2017b. Estimating wheat green area index from ground-based LiDAR measurement using a 3D canopy structure model. Agricultural and Forest Meteorology 247, 12-20.

Liu, Y., Liu, R., Chen, J.M., 2012. Retrospective retrieval of long-term consistent global leaf area index (1981-2011) from combined AVHRR and MODIS data. Journal of Geophysical Research: Biogeosciences 117.

Liu, Y., Xiao, J., Ju, W., Zhu, G., Wu, X., Fan, W., Li, D., Zhou, Y., 2018b. Satellite-derived LAI products exhibit large discrepancies and can lead to substantial uncertainty in simulated carbon and water fluxes. Remote Sensing of Environment 206, 174-188.

Lovell, J., Jupp, D., Newnham, G., Coops, N., Culvenor, D., 2005. Simulation study for finding optimal lidar acquisition parameters for forest height retrieval. Forest Ecology and Management 214, 398-412.

Lovell, J., Jupp, D.L., Culvenor, D., Coops, N., 2003. Using airborne and ground-based ranging lidar to measure canopy structure in Australian forests. Canadian Journal of Remote Sensing 29, 607-622.

Luo, S., Wang, C., Li, G., Xi, X., 2013. Retrieving leaf area index using ICESat/GLAS full-waveform data. Remote Sensing Letters 4, 745-753.

Ma, L., Zheng, G., Eitel, J.U., Magney, T.S., Moskal, L.M., 2017. Retrieving forest canopy extinction coefficient from terrestrial and airborne lidar. Agricultural and Forest Meteorology 236, 1-21.

Ma, Q., 2018. Quantifying forest structure parameters and their changes from LiDAR data and satellite imagery in the Sierra Nevada. Ph.D. thesis.

MacArthur, R.H., Horn, H.S., 1969. Foliage profile by vertical measurements. Ecology 50, 802-804.

MacArthur, R.H., MacArthur, J.W., 1961. On bird species diversity. Ecology 42, 594-598.

Macfarlane, C., 2011. Classification method of mixed pixels does not affect canopy metrics from digital images of forest overstorey. Agricultural and Forest Meteorology 151, 833-840.

Macfarlane, C., Grigg, A., Evangelista, C., 2007. Estimating forest leaf area using cover and fullframe fisheye photography: thinking inside the circle. Agricultural and Forest Meteorology 146, 1-12. 
Macfarlane, C., Ryu, Y., Ogden, G.N., Sonnentag, O., 2014. Digital canopy photography: exposed and in the raw. Agricultural and Forest Meteorology 197, 244-253.

Marino, E., Ranz, P., Tomé, J.L., Noriega, M.Á., Esteban, J., Madrigal, J., 2016. Generation of high-resolution fuel model maps from discrete airborne laser scanner and Landsat-8 OLI: A low-cost and highly updated methodology for large areas. Remote Sensing of Environment 187, 267-280.

Marselis, S.M., Tang, H., Armston, J.D., Calders, K., Labrière, N., Dubayah, R., 2018. Distinguishing vegetation types with airborne waveform lidar data in a tropical forest-savanna mosaic: A case study in Lopé National Park, Gabon. Remote Sensing of Environment 216, 626-634.

McElhinny, C., Gibbons, P., Brack, C., Bauhus, J., 2005. Forest and woodland stand structural complexity: its definition and measurement. Forest Ecology and Management 218, 1-24.

McMillen, G.G., McClendon, J.H., 1979. Leaf angle: an adaptive feature of sun and shade leaves. Botanical Gazette 140, 437-442.

McNeil, B.E., Pisek, J., Lepisk, H., Flamenco, E.A., 2016. Measuring leaf angle distribution in broadleaf canopies using UAVs. Agricultural and Forest Meteorology 218, 204-208.

Melgani, F., Bruzzone, L., 2004. Classification of hyperspectral remote sensing images with support vector machines. IEEE Transactions on Geoscience and Remote Sensing 42, 1778-1790.

Middleton, E., Huemmrich, K., Landis, D., Black, T., Barr, A., McCaughey, J., 2016. Photosynthetic efficiency of northern forest ecosystems using a MODIS-derived Photochemical Reflectance Index (PRI). Remote Sensing of Environment 187, 345-366.

Morsdorf, F., Kötz, B., Meier, E., Itten, K., Allgöwer, B., 2006. Estimation of LAI and fractional cover from small footprint airborne laser scanning data based on gap fraction. Remote Sensing of Environment 104, 50-61.

Morsdorf, F., Mårell, A., Koetz, B., Cassagne, N., Pimont, F., Rigolot, E., Allgöwer, B., 2010. Discrimination of vegetation strata in a multilayered Mediterranean forest ecosystem using height and intensity information derived from airborne laser scanning. Remote Sensing of Environment 114, 1403-1415.

Muller, J., Brandl, R., Buchner, J., Pretzsch, H., Seifert, S., Stratz, C., Veith, 
M., Fenton, B., 2013. From ground to above canopy-Bat activity in mature forests is driven by vegetation density and height. Forest Ecology and Management 306, 179-184.

Muller-Linow, M., Pinto-Espinosa, F., Scharr, H., Rascher, U., 2015. The leaf angle distribution of natural plant populations: assessing the canopy with a novel software tool. Plant Methods 11.

Muss, J.D., Mladenoff, D.J., Townsend, P.A., 2011. A pseudo-waveform technique to assess forest structure using discrete lidar data. Remote Sensing of Environment 115, 824-835.

Myneni, R.B., Hall, F.G., Sellers, P.J., Marshak, A.L., 1995. The interpretation of spectral vegetation indexes. IEEE Transactions on Geoscience and Remote Sensing 33, 481-486.

Myneni, R.B., Hoffman, S., Knyazikhin, Y., Privette, J., Glassy, J., Tian, Y., Wang, Y., Song, X., Zhang, Y., Smith, G., 2002. Global products of vegetation leaf area and fraction absorbed PAR from year one of MODIS data. Remote Sensing of Environment 83, 214-231.

Nanko, K., Mizugaki, S., Onda, Y., 2008. Estimation of soil splash detachment rates on the forest floor of an unmanaged Japanese cypress plantation based on field measurements of throughfall drop sizes and velocities. Catena 72, 348-361.

Newnham, G., Armston, J., Muir, J., Goodwin, N., Tindall, D., Culvenor, D., Püschel, P., Nyström, M., Johansen, K., 2012. Evaluation of terrestrial laser scanners for measuring vegetation structure. Australia: CSIRO .

Niinemets, l., 2010. A review of light interception in plant stands from leaf to canopy in different plant functional types and in species with varying shade tolerance. Ecological Research 25, 693-714.

Nilson, T., 1971. A theoretical analysis of the frequency of gaps in plant stands. Agricultural meteorology 8, 25-38.

Norman, J.M., Campbell, G.S., 1989. Canopy structure. Springer. pp. 301-325.

Ollinger, S., 2011. Sources of variability in canopy reflectance and the convergent properties of plants. New Phytologist 189, 375-394.

Oshio, H., Asawa, T., Hoyano, A., Miyasaka, S., 2015. Estimation of the leaf area density distribution of individual trees using high-resolution and multi-return airborne LiDAR data. Remote Sensing of Environment 166, 116-125.

Palace, M.W., Sullivan, F.B., Ducey, M.J., Treuhaft, R.N., Herrick, C., Shimbo, 
J.Z., Mota-E-Silva, J., 2015. Estimating forest structure in a tropical forest using field measurements, a synthetic model and discrete return lidar data. Remote Sensing of Environment 161, 1-11.

Pan, Y.D., Birdsey, R.A., Fang, J.Y., Houghton, R., Kauppi, P.E., Kurz, W.A., Phillips, O.L., Shvidenko, A., Lewis, S.L., Canadell, J.G., Ciais, P., Jackson, R.B., Pacala, S.W., McGuire, A.D., Piao, S.L., Rautiainen, A., Sitch, S., Hayes, D., 2011. A Large and Persistent Carbon Sink in the World's Forests. Science 333, 988-993.

Pang, Y., Lefsky, M., Sun, G., Ranson, J., 2011. Impact of footprint diameter and off-nadir pointing on the precision of canopy height estimates from spaceborne lidar. Remote Sensing of Environment 115, 2798-2809.

Parker, G.G., Lefsky, M.A., Harding, D.J., 2001. Light transmittance in forest canopies determined using airborne laser altimetry and incanopy quantum measurements. Remote Sensing of Environment 76, 298-309.

Pearcy, R.W., Valladares, F., Wright, S.J., De Paulis, E.L., 2004. A functional analysis of the crown architecture of tropical forest Psychotria species: do species vary in light capture efficiency and consequently in carbon gain and growth? Oecologia 139, 163-177.

Pereira, H.M., Ferrier, S., Walters, M., Geller, G.N., Jongman, R., Scholes, R.J., Bruford, M.W., Brummitt, N., Butchart, S., Cardoso, A., 2013. Essential biodiversity variables. Science 339, 277-278.

Perez, R.P.A., Costes, E., Theveny, F., Griffon, S., Caliman, J.P., Dauzat, J., 2018. 3D plant model assessed by terrestrial LiDAR and hemispherical photographs: A useful tool for comparing light interception among oil palm progenies. Agricultural and Forest Meteorology 249, 250-263.

Pfennigbauer, M., Ullrich, A., 2010. Improving quality of laser scanning data acquisition through calibrated amplitude and pulse deviation measurement. Laser Radar Technology and Applications Xv 7684.

Piayda, A., Dubbert, M., Werner, C., Correia, A.V., Pereira, J.S., Cuntz, M., 2015. Influence of woody tissue and leaf clumping on vertically resolved leaf area index and angular gap probability estimates. Forest Ecology and Management 340, 103-113.

Pisek, J., Ryu, Y., Alikas, K., 2011. Estimating leaf inclination and Gfunction from leveled digital camera photography in broadleaf canopies. Trees 25, 919-924. 
Pisek, J., Sonnentag, O., Richardson, A.D., M?ttus, M., 2013. Is the spherical leaf inclination angle distribution a valid assumption for temperate and boreal broadleaf tree species? Agricultural and Forest Meteorology 169, 186-194.

Planchais, I., Pontailler, J.Y., 1999. Validity of leaf areas and angles estimated in a beech forest from analysis of gap frequencies, using hemispherical photographs and a plant canopy analyzer. Annals of Forest Science 56, 1-10.

Prescott, C.E., 2002. The influence of the forest canopy on nutrient cycling. Tree Physiology 22, 1193-1200.

Pueschel, P., Buddenbaum, H., Hill, J., 2012. An efficient approach to standardizing the processing of hemispherical images for the estimation of forest structural attributes. Agricultural and Forest Meteorology 160, 1-13.

Raabe, K., Pisek, J., Sonnentag, O., Annuk, K., 2015. Variations of leaf inclination angle distribution with height over the growing season and light exposure for eight broadleaf tree species. Agricultural and Forest Meteorology 214, 2-11.

Rautiainen, M., Stenberg, P., 2015. On the angular dependency of canopy gap fractions in pine, spruce and birch stands. Agricultural and Forest Meteorology 206, 1-3.

Riaño, D., Valladares, F., Condés, S., Chuvieco, E., 2004. Estimation of leaf area index and covered ground from airborne laser scanner (Lidar) in two contrasting forests. Agricultural and Forest Meteorology 124, 269-275.

Richardson, J.J., Moskal, L.M., Kim, S.H., 2009. Modeling approaches to estimate effective leaf area index from aerial discrete-return LIDAR. Agricultural and Forest Meteorology 149, 1152-1160.

Ross, J., 1981. The radiation regime and architecture of plant stands. Springer Science \& Business Media.

Rrnyi, A., 1961. On measures of entropy and information, in: Fourth Berkeley symposium on mathematical statistics and probability, pp. 547-561.

Ryu, Y., Baldocchi, D.D., Kobayashi, H., Ingen, C., Li, J., Black, T.A., Beringer, J., Gorsel, E., Knohl, A., Law, B.E., 2011. Integration of MODIS land and atmosphere products with a coupled-process model to estimate gross primary productivity and evapotranspiration from $1 \mathrm{~km}$ to 
global scales. Global Biogeochemical Cycles 25.

Ryu, Y., Sonnentag, O., Nilson, T., Vargas, R., Kobayashi, H., Wenk, R., Baldocchi, D.D., 2010. How to quantify tree leaf area index in an open savanna ecosystem: a multi-instrument and multi-model approach. Agricultural and Forest Meteorology 150, 63-76.

Saatchi, S.S., Harris, N.L., Brown, S., Lefsky, M., Mitchard, E.T.A., Salas, W., Zutta, B.R., Buermann, W., Lewis, S.L., Hagen, S., Petrova, S., White, L., Silman, M., Morel, A., 2011. Benchmark map of forest carbon stocks in tropical regions across three continents. Proceedings of the National Academy of Sciences of the United States of America 108, 9899-9904. Sakamoto, T., Morinaka, Y., Ohnishi, T., Sunohara, H., Fujioka, S., UeguchiTanaka, M., Mizutani, M., Sakata, K., Takatsuto, S., Yoshida, S., 2006. Erect leaves caused by brassinosteroid deficiency increase biomass production and grain yield in rice. Nature Biotechnology 24, 105.

San-Miguel-Ayanz, J., de Rigo, D., Caudullo, G., Durrant, T.H., Mauri, A., Tinner, W., Ballian, D., Beck, P., Birks, H., Eaton, E., 2016. European atlas of forest tree species .

Schleppi, P., Conedera, M., Sedivy, I., Thimonier, A., 2007. Correcting non-linearity and slope effects in the estimation of the leaf area index of forests from hemispherical photographs. Agricultural and Forest Meteorology 144, 236-242.

Schnitzer, S.A., Carson, W.P., 2001. Treefall gaps and the maintenance of species diversity in a tropical forest. Ecology 82, 913-919.

Seidel, D., Fleck, S., Leuschner, C., 2012. Analyzing forest canopies with ground-based laser scanning: a comparison with hemispherical photography. Agricultural and Forest Meteorology 154, 1-8.

Senf, C., Pflugmacher, D., Zhiqiang, Y., Sebald, J., Knorn, J., Neumann, M., Hostert, P., Seidl, R., 2018. Canopy mortality has doubled in Europe's temperate forests over the last three decades. Nature Communications 9, 4978.

Shell, G., Lang, A., Sale, P., 1974. Quantitative measures of leaf orientation and heliotropic response in sunflower, bean, pepper and cucumber. Agricultural meteorology 13, 25-37.

Shine, R., Barrott, E.G., Elphick, M.J., 2002. Some like it hot: effects of forest clearing on nest temperatures of montane reptiles. Ecology 83, 2808-2815.

Shugart, H., Saatchi, S., Hall, F., 2010. Importance of structure and its 
measurement in quantifying function of forest ecosystems. Journal of Geophysical Research: Biogeosciences 115.

Siegel, R.B., DeSante, D.F., 2003. Bird communities in thinned versus unthinned Sierran mixed conifer stands. The Wilson Bulletin , 155-165.

Silveyra Gonzalez, R., Latifi, H., Weinacker, H., Dees, M., Koch, B., Heurich, M., 2018. Integrating LiDAR and high-resolution imagery for objectbased mapping of forest habitats in a heterogeneous temperate forest landscape. International Journal of Remote Sensing , 1-26.

Simard, M., Pinto, N., Fisher, J.B., Baccini, A., 2011. Mapping forest canopy height globally with spaceborne lidar. Journal of Geophysical Research: Biogeosciences 116.

Sinoquet, H., Thanisawanyangkura, S., Mabrouk, H., Kasemsap, P., 1998. Characterization of the light environment in canopies using 3D digitising and image processing. Annals of Botany 82, 203-212.

Skidmore, A.K., Pettorelli, N., 2015. Agree on biodiversity metrics to track from space: ecologists and space agencies must forge a global monitoring strategy. Nature 523, 403-406.

Solberg, S., Brunner, A., Hanssen, K.H., Lange, H., Næsset, E., Rautiainen, M., Stenberg, P., 2009. Mapping LAI in a Norway spruce forest using airborne laser scanning. Remote Sensing of Environment 113, 2317 2327.

Solberg, S., Næsset, E., Hanssen, K.H., Christiansen, E., 2006. Mapping defoliation during a severe insect attack on Scots pine using airborne laser scanning. Remote Sensing of Environment 102, 364-376.

Stark, S.C., Enquist, B.J., Saleska, S.R., Leitold, V., Schietti, J., Longo, M., Alves, L.F., Camargo, P.B., Oliveira, R.C., 2015. Linking canopy leaf area and light environments with tree size distributions to explain Amazon forest demography. Ecology Letters 18, 636-645.

Stark, S.C., Leitold, V., Wu, J.L., Hunter, M.O., de Castilho, C.V., Costa, F.R., McMahon, S.M., Parker, G.G., Shimabukuro, M.T., Lefsky, M.A., 2012. Amazon forest carbon dynamics predicted by profiles of canopy leaf area and light environment. Ecology letters 15, 1406-1414.

Su, J., Bork, E., 2006. Influence of vegetation, slope, and lidar sampling angle on DEM accuracy. Photogrammetric Engineering \& Remote Sensing 72, 1265-1274.

Sugarbaker, L., Constance, E.W., Heidemann, H.K., Jason, A.L., Lucas, V., Saghy, D., Stoker, J.M., 2014. The 3D Elevation Program initiative: a 
call for action. US Geological Survey.

Tang, H., 2015. Lidar remote sensing of vertical foliage profile and leaf area index. Ph.D. thesis.

Tang, H., Brolly, M., Zhao, F., Strahler, A.H., Schaaf, C.L., Ganguly, S., Zhang, G., Dubayah, R., 2014a. Deriving and validating Leaf Area Index (LAI) at multiple spatial scales through lidar remote sensing: A case study in Sierra National Forest, CA. Remote Sensing of Environment 143, 131-141.

Tang, H., Dubayah, R., 2017. Light-driven growth in Amazon evergreen forests explained by seasonal variations of vertical canopy structure. Proceedings of the National Academy of Sciences , 201616943.

Tang, H., Dubayah, R., Brolly, M., Ganguly, S., Zhang, G., 2014b. Largescale retrieval of leaf area index and vertical foliage profile from the spaceborne waveform lidar (GLAS/ICESat). Remote Sensing of Environment 154, 8-18.

Tang, H., Dubayah, R., Swatantran, A., Hofton, M., Sheldon, S., Clark, D.B., Blair, B., 2012. Retrieval of vertical LAI profiles over tropical rain forests using waveform lidar at La Selva, Costa Rica. Remote Sensing of Environment 124, 242-250.

Tang, H., Ganguly, S., Zhang, G., Hofton, M.A., Nelson, R.F., Dubayah, R., 2016. Characterizing leaf area index (LAI) and vertical foliage profile (VFP) over the United States. Biogeosciences 13, 239-252.

Tateno, R., Hishi, T., Takeda, H., 2004. Above-and belowground biomass and net primary production in a cool-temperate deciduous forest in relation to topographical changes in soil nitrogen. Forest Ecology and Management 193, 297-306.

Tews, J., Brose, U., Grimm, V., Tielbörger, K., Wichmann, M., Schwager, M., Jeltsch, F., 2004. Animal species diversity driven by habitat heterogeneity/diversity: the importance of keystone structures. Journal of Biogeography 31, 79-92.

Van der Tol, C., Verhoef, W., Timmermans, J., Verhoef, A., Su, Z., 2009. An integrated model of soil-canopy spectral radiances, photosynthesis, fluorescence, temperature and energy balance. Biogeosciences 6, 31093129.

Treuhaft, R.N., Chapman, B.D., Dos Santos, J., Gonçalves, F.G., Dutra, L.V., Graça, P., Drake, J.B., 2009. Vegetation profiles in tropical forests from multibaseline interferometric synthetic aperture radar, field, and lidar 
measurements. Journal of Geophysical Research: Atmospheres 114.

Utsugi, H., Araki, M., Kawasaki, T., Ishizuka, M., 2006. Vertical distributions of leaf area and inclination angle, and their relationship in a 46-year-old Chamaecyparis obtusa stand. Forest Ecology and Management 225, 104-112.

Valladares, F., Gianoli, E., GÃşmez, J.M., 2007. Ecological limits to plant phenotypic plasticity. New phytologist 176, 749-763.

Véga, C., Durrieu, S., 2011. Multi-level filtering segmentation to measure individual tree parameters based on Lidar data: Application to a mountainous forest with heterogeneous stands. International Journal of Applied Earth Observation and Geoinformation 13, 646-656.

Verhoef, W., 1984. Light scattering by leaf layers with application to canopy reflectance modeling: the SAIL model. Remote Sensing of Environment 16, 125-141.

Vierling, K.T., Vierling, L.A., Gould, W.A., Martinuzzi, S., Clawges, R.M., 2008. Lidar: shedding new light on habitat characterization and modeling. Frontiers in Ecology and the Environment 6, 90-98.

Vincent, G., Antin, C., Laurans, M., Heurtebize, J., Durrieu, S., Lavalley, C., Dauzat, J., 2017. Mapping plant area index of tropical evergreen forest by airborne laser scanning. A cross-validation study using LAI2200 optical sensor. Remote Sensing of Environment 198, 254-266.

Wagner, S., Hagemeier, M., 2006. Method of segmentation affects leaf inclination angle estimation in hemispherical photography. Agricultural and Forest Meteorology 139, 12-24.

Wagner, W., Ullrich, A., Ducic, V., Melzer, T., Studnicka, N., 2006. Gaussian decomposition and calibration of a novel small-footprint full-waveform digitising airborne laser scanner. ISPRS Journal of Photogrammetry and Remote Sensing 60, 100-112.

Walther, B.A., 2002. Vertical stratification and use of vegetation and light habitats by Neotropical forest birds. Journal für Ornithologie 143, 64-81.

Wang, Q., Li, P.H., 2013. Canopy vertical heterogeneity plays a critical role in reflectance simulation. Agricultural and Forest Meteorology $169,111-121$.

Wang, W.M., Li, Z.L., Su, H.B., 2007. Comparison of leaf angle distribution functions: Effects on extinction coefficient and fraction of sunlit foliage. Agricultural and Forest Meteorology 143, 106-122. 
Weishampel, J.F., Blair, J.B., Knox, R., Dubayah, R., Clark, D., 2000. Volumetric lidar return patterns from an old-growth tropical rainforest canopy. International Journal of Remote Sensing 21, 409-415.

Weiss, M., Baret, F., 2010. CAN-EYE V6. 1 user manual. INRA, Paris, France

Weiss, M., Baret, F., Garrigues, S., Lacaze, R., 2007. LAI and fAPAR CYCLOPES global products derived from VEGETATION. Part 2: validation and comparison with MODIS collection 4 products. Remote Sensing of Environment 110, 317-331.

Weiss, M., Baret, F., Smith, G., Jonckheere, I., Coppin, P., 2004. Review of methods for in situ leaf area index (LAI) determination: Part II. Estimation of LAI, errors and sampling. Agricultural and Forest Meteorology 121, 37-53.

Welles, J.M., 1990. Some indirect methods of estimating canopy structure. Remote Sensing Reviews 5, 31-43.

Whitehurst, A.S., Swatantran, A., Blair, J.B., Hofton, M.A., Dubayah, R., 2013. Characterization of canopy layering in forested ecosystems using full waveform lidar. Remote Sensing 5, 2014-2036.

Widlowski, J.L., Taberner, M., Pinty, B., Bruniquel-Pinel, V., Disney, M., Fernandes, R., Gastellu-Etchegorry, J.P., Gobron, N., Kuusk, A., Lavergne, T., et al., 2007. Third Radiation Transfer Model Intercomparison (RAMI) exercise: Documenting progress in canopy reflectance models 112 , 139-155.

Wilkes, P., Jones, S.D., Suarez, L., Haywood, A., Mellor, A., Woodgate, W., Soto-Berelov, M., Skidmore, A.K., 2015a. Using discrete-return airborne laser scanning to quantify number of canopy strata across diverse forest types. Methods in Ecology and Evolution .

Wilkes, P., Jones, S.D., Suarez, L., Haywood, A., Woodgate, W., SotoBerelov, M., Mellor, A., Skidmore, A.K., 2015b. Understanding the Effects of ALS Pulse Density for Metric Retrieval across Diverse Forest Types. Photogrammetric Engineering and Remote Sensing 81, 625-635.

Wilkes, P., Lau, A., Disney, M., Calders, K., Burt, A., de Tanago, J.G., Bartholomeus, H., Brede, B., Herold, M., 2017. Data acquisition considerations for Terrestrial Laser Scanning of forest plots. Remote Sensing of Environment 196, 140-153.

Wilson, J.W., 1960. Inclined point quadrats. New Phytologist 59, 1-7.

Wilson, J.W., 1963. Estimation of foliage denseness and foliage angle by 
inclined point quadrats. Australian Journal of Botany 11, 95-105.

Wilson, K.B., Hanson, P.J., Mulholland, P.J., Baldocchi, D.D., Wullschleger, S.D., 2001. A comparison of methods for determining forest evapotranspiration and its components: sap-flow, soil water budget, eddy covariance and catchment water balance. Agricultural and Forest Meteorology 106, 153-168.

Wirth, R., Weber, B., Ryel, R.J., 2001. Spatial and temporal variability of canopy structure in a tropical moist forest. Acta Oecologica 22, 235-244.

de Wit, C.T., 1965. Photosynthesis of leaf canopies. Report. Pudoc.

Woodgate, W., Armston, J.D., Disney, M., Suarez, L., Jones, S.D., Hill, M.J., Wilkes, P., Soto-Berelov, M., 2017. Validating canopy clumping retrieval methods using hemispherical photography in a simulated Eucalypt forest. Agricultural and Forest Meteorology 247, 181-193.

Woodgate, W., Jones, S.D., Suarez, L., Hill, M.J., Armston, J.D., Wilkes, P., Soto-Berelov, M., Haywood, A., Mellor, A., 2015. Understanding the variability in ground-based methods for retrieving canopy openness, gap fraction, and leaf area index in diverse forest systems. Agricultural and Forest Meteorology 205, 83-95.

Wulder, M.A., White, J.C., Nelson, R.F., Næsset, E., Ørka, H.O., Coops, N.C., Hilker, T., Bater, C.W., Gobakken, T., 2012. Lidar sampling for largearea forest characterization: A review. Remote Sensing of Environment $121,196-209$

Xiao, Q., McPherson, E.G., Ustin, S.L., Grismer, M.E., 2000. A new approach to modeling tree rainfall interception. Journal of Geophysical Research: Atmospheres 105, 29173-29188.

Yan, K., Park, T., Yan, G., Chen, C., Yang, B., Liu, Z., Nemani, R.R., Knyazikhin, Y., Myneni, R.B., 2016. Evaluation of MODIS LAI/FPAR product collection 6. Part 1: Consistency and improvements. Remote Sensing 8, 359.

Yang, P., Verhoef, W., Van Der Tol, C., 2017. The mSCOPE model: A simple adaptation to the SCOPE model to describe reflectance, fluorescence and photosynthesis of vertically heterogeneous canopies. Remote Sensing of Environment 201, 1-11.

Zhang, W., Qi, J., Wan, P., Wang, H., Xie, D., Wang, X., Yan, G., 2016. An Easy-to-Use Airborne LiDAR Data Filtering Method Based on Cloth Simulation. Remote Sensing 8, 501. 
Zhang, Y., Chen, J.M., Miller, J.R., 2005. Determining digital hemispherical photograph exposure for leaf area index estimation. Agricultural and Forest Meteorology 133, 166-181.

Zhao, F., Yang, X., Schull, M.A., Román-Colón, M.O., Yao, T., Wang, Z., Zhang, Q., Jupp, D.L., Lovell, J.L., Culvenor, D.S., et al., 2011. Measuring effective leaf area index, foliage profile, and stand height in New England forest stands using a full-waveform ground-based lidar. Remote Sensing of Environment 115, 2954-2964.

Zhao, K.G., Garcia, M., Liu, S., Guo, Q.H., Chen, G., Zhang, X.S., Zhou, Y.Y., Meng, X.L., 2015. Terrestrial lidar remote sensing of forests: Maximum likelihood estimates of canopy profile, leaf area index, and leaf angle distribution. Agricultural and Forest Meteorology 209, 100-113.

Zhao, K.G., Popescu, S., 2009. Lidar-based mapping of leaf area index and its use for validating GLOBCARBON satellite LAI product in a temperate forest of the southern USA. Remote Sensing of Environment $113,1628-1645$.

Zhao, X., Liang, S., Liu, S., Yuan, W., Xiao, Z., Liu, Q., Cheng, J., Zhang, X., Tang, H., Zhang, X., et al., 2013. The Global Land Surface Satellite (GLASS) remote sensing data processing system and products. Remote Sensing 5, 2436-2450.

Zheng, G., Ma, L., Eitel, J.U., He, W., Magney, T.S., Moskal, L.M., Li, M., 2017. Retrieving Directional Gap Fraction, Extinction Coefficient, and Effective Leaf Area Index by Incorporating Scan Angle Information From Discrete Aerial Lidar Data. IEEE Transactions on Geoscience and Remote Sensing 55, 577-590.

Zheng, G., Moskal, L.M., 2009. Retrieving Leaf Area Index (LAI) Using Remote Sensing: Theories, Methods and Sensors. Sensors 9, 27192745.

Zheng, G., Moskal, L.M., 2012. Leaf orientation retrieval from terrestrial laser scanning (TLS) data. IEEE Transactions on Geoscience and Remote Sensing 50, 3970-3979.

Zhu, X., Skidmore, A.K., Darvishzadeh, R., Niemann, K.O., Liu, J., Shi, Y., Wang, T., 2018a. Foliar and woody materials discriminated using terrestrial LiDAR in a mixed natural forest. International Journal of Applied Earth Observation and Geoinformation 64, 43-50.

Zhu, X., Skidmore, A.K., Wang, T., Liu, J., Darvishzadeh, R., Shi, Y., Premier, J., Heurich, M., 2018b. Improving leaf area index (LAI) estimation 
by correcting for clumping and woody effects using terrestrial laser scanning. Agricultural and Forest Meteorology 263, 276-286.

Zhu, X., Wang, T., Skidmore, A.K., Darvishzadeh, R., Niemann, K.O., Liu, J., 2017. Canopy leaf water content estimated using terrestrial LiDAR. Agricultural and Forest Meteorology 232, 152-162.

Zou, J., Zhuang, Y., Chianucci, F., Mai, C., Lin, W., Leng, P., Luo, S., Yan, B., 2018. Comparison of Seven Inversion Models for Estimating Plant and Woody Area Indices of Leaf-on and Leaf-off Forest Canopy Using Explicit 3D Forest Scenes. Remote Sensing 10, 1297. 


\section{Summary}

In forests, leaves are the interface between the biosphere and the atmosphere, where most of the energy fluxes exchange. The three dimensional (3D) distribution of leaves strongly influence the interception and distribution of radiation, carbon and water. Leaf area index (LAI) and the vertical LAI profile are important metrics describing the amount and distribution of leaves. LAI has been identified as an essential climate variable and an essential biodiversity variable. The vertical LAI profile is a more detailed description of the 3D distribution of leaves inside the canopy. Both metrics have been used in ecology, hydrology and biodiversity modelling.

Remote sensing techniques provide a non-destructive, rapid and economic way for estimating LAI and vertical LAI profile across a wide range of spatial and temporal scales. However, passive optical sensors suffer from limitations including signal saturation and inability to resolve the vertical distribution. As a result, LiDAR plays an indispensable role in mapping the 3D distribution of leaves in forests. Nevertheless, accurate 3D mapping of leaves is a challenging task for forest ecosystem, owing to the complexity of canopy structure, underlying topography, and LiDAR settings.

This thesis evaluated several key factors in the LAI and vertical LAI profile retrieval using LiDAR data at local and regional scale. These factors include the leaf angle distribution (LAD), gap fraction, LiDAR scan angle, and uneven topography, all of which were parameters in the physically based gap fraction model to estimate LAI and vertical LAI profile. At local scale, the thesis first examined which in-situ technique produced more accurate LAD estimate. Using field-based and simulation dataset, terrestrial LiDAR was proved more accurate than DHP when estimating 
LAD in broadleaf forests. Then the thesis employed the proposed LAD method to examine whether the spherical LAD assumption was valid for natural European beech forests. Using terrestrial LiDAR, large LAD variation was demonstrated both in different stands and in different canopy layers. A uniform distribution rather than a spherical distribution was a more valid LAD assumption. At regional scale, the thesis evaluated the effect of airborne LiDAR flight settings, in particular the scan angle, on the retrieval of gap fraction and vertical gap fraction profile. The results proved that the underestimation of gap fraction amplified at large off-nadir scan angle. It implied that large off-nadir scan angle LiDAR data should be avoided to ensure a more accurate gap fraction and LAI retrieval. Finally, the thesis assessed the effect of uneven topography and topographic normalization in the vertical LAI profile retrieval. The findings demonstrated that topographic normalization undermined the complexity of the vertical LAI profile. For ecological applications, such as biodiversity modeling, topographic normalization was suggested not to be applied.

The new methodologies and findings in the study can be extended to other forests on different sites or of different species. Further studies are recommended to explore the application of LiDAR derived LAI and vertical LAI profile product in modelling carbon stock, forest dynamics and biodiversity. 


\section{Samenvatting}

In bossen zijn bladeren het grensvlak tussen de biosfeer en de atmosfeer, waar de meeste energiestromen wisselen. De driedimensionale (3D) verdeling van bladeren heeft een sterke invloed op de onderschepping en verspreiding van straling, koolstof en water. Bladoppervlakte-index (LAI) en het verticale LAI-profiel zijn belangrijke meetwaarden die de hoeveelheid en verdeling van bladeren beschrijven. LAI is geÃŕdentificeerd als een essentiÃńle klimaatvariabele en een essentiÃńle biodiversiteitsvariabele. Het verticale LAI-profiel is een meer gedetailleerde beschrijving van de 3D-verdeling van bladeren in de overkapping. Beide metrieken zijn gebruikt in de modellering van ecologie, hydrologie en biodiversiteit.

Remote sensing-technieken bieden een niet-destructieve, snelle en economische manier voor het schatten van LAI en verticaal LAI-profiel op een breed scala van ruimtelijke en temporele schalen. Passieve optische sensoren lijden echter aan beperkingen, waaronder signaalverzadiging en onvermogen om de verticale verdeling op te lossen. Als gevolg hiervan speelt LiDAR een onmisbare rol bij het in kaart brengen van de 3D-verdeling van bladeren in bossen. Niettemin is het nauwkeurig 3Dmappen van bladeren een uitdagende taak voor het bosecosysteem, vanwege de complexiteit van de structuur van de luifel, de onderliggende topografie en de LiDAR-instellingen.

Dit proefschrift evalueerde verschillende sleutelfactoren in het LAI en het ophalen van verticale LAI-profielen met behulp van LiDAR-gegevens op lokale en regionale schaal. Deze factoren omvatten de bladhoekverdeling (LAD), spleetfractie, LiDAR-scanhoek en ongelijke topografie, die allemaal parameters waren in het fysisch gebaseerde gap-fractiemodel om LAI en verticaal LAI-profiel te schatten. Op lokale schaal onderzocht het proefschrift eerst welke in-situ techniek een nauwkeurigere LAD- 
schatting opleverde. Met behulp van een veldgebaseerde en simulatieset, bleek terrestrische LiDAR nauwkeuriger dan DHP bij het schatten van LAD in breedbladige bossen. Vervolgens gebruikte het proefschrift de voorgestelde LAD-methode om te onderzoeken of de sferische LADaanname geldig was voor natuurlijke Europese beukenbossen. Met behulp van terrestrische LiDAR werd grote LAD-variatie zowel in verschillende stands als in verschillende luifellagen gedemonstreerd. Een meer uniforme verdeling in plaats van een sferische verdeling was een meer geldige veronderstelling van de LAD. Op regionale schaal evalueerde het proefschrift het effect van in de lucht vliegende LiDAR-vluchtinstellingen, in het bijzonder de scanhoek, op het ophalen van spleetfractie en verticaal gapfractieprofiel. De resultaten bewezen dat de onderschatting van de gap-fractie versterkt werd bij een grote off-nadir scanhoek. Dit hield in dat grote LDAR-gegevens van de dalpuntscan moeten worden vermeden om een nauwkeuriger gap-breuk en LAI-terugwinning te garanderen. Ten slotte beoordeelde het proefschrift het effect van ongelijke topografie en topografische normalisatie in het ophalen van verticale LAI-profielen. De bevindingen toonden aan dat topografische normalisatie de complexiteit van het verticale LAI-profiel ondermijnde. Voor ecologische toepassingen, zoals biodiversiteitsmodellering, werd geopperd om topografische normalisatie niet toe te passen.

De nieuwe methodologieën en bevindingen in de studie kunnen worden uitgebreid naar andere bossen op verschillende locaties of van verschillende soorten. Verdere studies worden aanbevolen om de toepassing te onderzoeken van LiDAR afgeleid LAI en verticaal LAI profielproduct in het modelleren van koolstofvoorraad, bosdynamiek en biodiversiteit. 


\section{Author's biography and publications}

Jing Liu was born on $2^{\text {nd }}$ September 1989 in Shaanxi Province, China. She obtained the Bachelor of Science degree in Geographical Information Science from Nanjing University, China in 2011. In 2014, she graduated from Peking University with a Master of Science degree in Photogrammetry Engineering and Remote Sensing. From September 2014, she began pursuing her PhD degree in Natural Resources deparment of ITC, University of Twente and RMIT University. Her research interests include remote sensing of vegetation, LiDAR, and land use dynamics monitoring. 


\section{ISI journal publications}

Liu, J., Skidmore, A.K., Wang, T., Zhu, X., Premier, J., Heurich, M., Beudert, B., Jones, S., 2018. Variation of leaf angle distribution quantified by terrestrial LiDAR in natural European beech forests. ISPRS Journal of Photogrammetry and Remote Sensing, 148, 208-220.

Liu, J., Skidmore, A.K., Jones, S., Wang, T., Heurich, M., Zhu, X., Shi, Y., 2018. Large off-nadir scan angle of airborne LiDAR can severely affect the estimates of forest structure metrics. ISPRS Journal of Photogrammetry and Remote Sensing, 136, 13-25.

Liu, J., Skidmore, A.K., Heurich, M., Wang, T., 2017. Significant effect of topographic normalization of airborne LiDAR data on the retrieval of plant area index profile in mountainous forests. ISPRS Journal of Photogrammetry and Remote Sensing, 132, 77-87.

Liu, J., Li, P., Wang, X., 2015. A new segmentation method for very high resolution imagery using spectral and morphological information. ISPRS Journal of Photogrammetry and Remote Sensing, 101, 145-162.

Liu, J., Skidmore, A.K., Wang, T., Jones, S., Heurich, M., 2018. Comparison of terrestrial LiDAR and digital hemispherical photography in estimating leaf angle distribution in broadleaf forests. (to be submitted).

Zhu, X., Skidmore, A.K., Wang, T., Liu, J., Darvishzadeh, R., Shi, Y., Premier, J., Heurich, M., 2018. Improving leaf area index (LAI) estimation by correcting for clumping and woody effects using terrestrial laser scanning. Agricultural and Forest Meteorology, 263, 276-286.

Zhu, X., Skidmore, A.K., Darvishzadeh., R., Niemann, K., Liu, J., Shi, Y., Wang, T., 2018. Foliar and woody materials discriminated using terrestrial LiDAR in a mixed natural forest. International Journal of Applied Earth Observation and Geoinformation, 64, 43-50.

Zhu, X., Wang, T., Skidmore, A.K., Darvishzadeh., R., Niemann, K., Liu, J., 2017. Canopy leaf water content estimated using terrestrial LiDAR. Agricultural and Forest Meteorology, 232, 152-162 